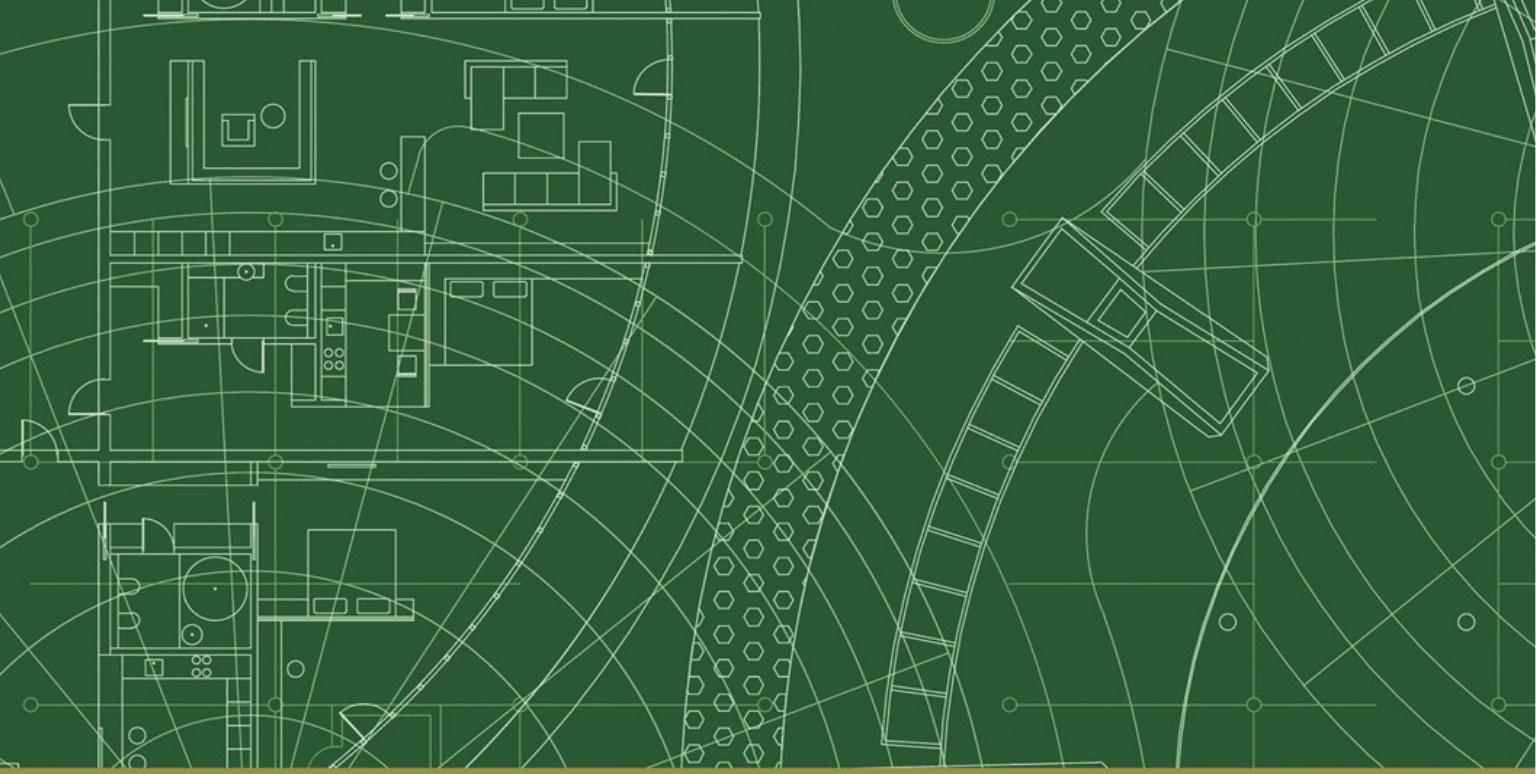

Routledge Studies in Innovation, Organizations and Technology

\title{
CITIZEN ACTIVITIES IN ENERGY TRANSITION
}

\section{USER INNOVATION, NEW COMMUNITIES, AND THE SHAPING OF A SUSTAINABLE FUTURE}

Sampsa Hyysalo
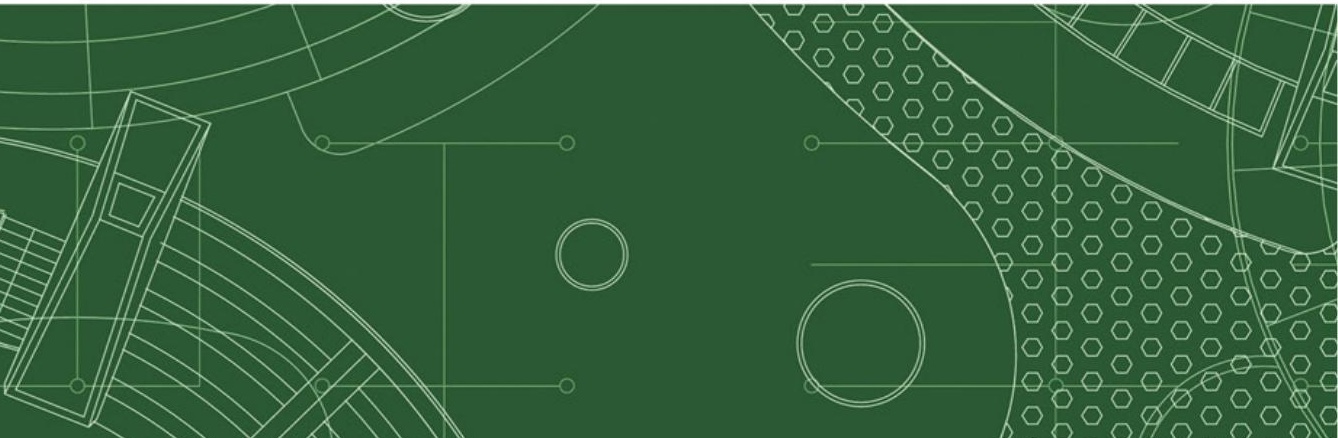


\section{Citizen Activities in Energy Transition}

This book addresses the rapidly changing citizen roles in innovation, technology adoption, intermediation, market creation, and legitimacy building for low-carbon solutions. It links research in innovation studies, sustainability transitions, and science and technology studies, and builds a new approach for the study of user contributions to innovation and sociotechnical change.

Citizen Activities in Energy Transition gives a detailed and empirically grounded overall appraisal of citizens' active technological engagement in the current energy transition, in an era when internet connectivity has given rise to important new forms of citizen communities and interactions. It elaborates a new way to study users in sociotechnical change through long-term ethnographic and historical research and reports its deployment in a major, decade-long line of investigation on user activities in small-scale renewables, addressing user contributions from the early years to the late proliferation stages of small-scale renewable energy technologies (S-RETs). It offers a muchneeded empirical and theoretical understanding of the dynamics of the activities in which users are engaged over the course of sociotechnical change, including innovation, adoption, adjustment, intermediation, community building, digital communities, market creation, and legitimacy creation.

This work is a must-read for those seeking to understand the role of users in innovation, energy systems change and the significance of new digital communities in present and future sociotechnical change. Academics, policymakers, and managers are given a new resource to understand the "demand side" of sociotechnical change beyond the patterns of investment, adoption, and social acceptance that have traditionally occupied their attention.

Sampsa Hyysalo is Professor of Co-Design at the Aalto University School of Art, Design and Architecture in Helsinki, Finland. His research focuses on designer-user relations in sociotechnical change. This includes engagement in participatory design, co-design, open and user innovation, open design, peer knowledge creation, user communities, citizen science, and user knowledge in organizations. His research orientation is multidisciplinary with science and technology studies, innovation studies, and collaborative design being 
his main fields. He has authored several books, the latest being The New Production of Users: Changing Innovation Communities and Involvement Strategies (written with Elgaard Jensen and Oudshoorn), which won the European Association for the Study of Science and Technology's Freeman Award, and Health Technology Development and Use: From Practice-Bound Imagination to Evolving Impacts (2010). Sampsa has published over 70 full-length articles and book chapters, including over ten publications in field-leading journals such as Research Policy, MIS Quarterly, Design Studies, and Social Studies of Science. He was the Chief Editor of Science \& Technology Studies journal 20072016 and was awarded the Academy of Finland Award for Social Impact in 2010 . 
"In this excellent book Sampsa Hyysalo describes how consumers affect sociotechnical change processes through innovation, peer help, market creation activities and shaping of public discourse. The specific area he studies in-depth is consumer innovation in renewable energy and how it is catalyzed by internet communities. The conclusions he draws are generally important to innovation scholars, innovation managers, and to innovation policymakers as well."

-Eric von Hippel, MIT

"Finally: a comprehensive exposition of how users participate in sociotechnical change."

—Eva Heiskanen, University of Helsinki

"This carefully researched book surfaces the invisible work that citizens do in procuring, installing and improving new energy technologies, and how this greatly matters regarding the shape of technologies and markets."

-Robin Williams, University of Edinburgh 


\section{R101!}

Routledge Studies in Innovation, Organizations and Technology

Developing Capacity for Innovation in Complex Systems

Strategy, Organisation and Leadership

Christer Vindeløv-Lidzélius

How is Digitalization Affecting Agri-food?

New Business Models, Strategies and Organizational Forms

Edited by Maria Carmela Annosi and Federica Brunetta

Social Innovation of New Ventures

Achieving Social Inclusion and Sustainability in Emerging Economies and Developing Countries

Marcela Ramírez-Pasillas, Vanessa Ratten and Hans Lundberg

\section{Sustainable Innovation}

Strategy, Process and Impact

Edited by Cosmina L. Voinea, Nadine Roijakkers and Ward Ooms

\section{Management in the Age of Digital Business Complexity}

Edited by Bill McKelvey

\section{Citizen Activities in Energy Transition}

User Innovation, New Communities, and the Shaping of a Sustainable Future Sampsa Hyysalo

For more information about this series, please visit: www.routledge.com/ Routledge-Studies-in-Innovation-Organizations-and-Technology/bookseries/RIOT 


\section{Citizen Activities in Energy Transition \\ User Innovation, New Communities, and the Shaping of a Sustainable Future}

Sampsa Hyysalo 
First published 2021

by Routledge

2 Park Square, Milton Park, Abingdon, Oxon OX14 4RN

and by Routledge

605 Third Avenue, New York, NY 10158

Routledge is an imprint of the Taylor \& Francis Group, an informa business

(C) 2021 Sampsa Hyysalo

The right of Sampsa Hyysalo to be identified as author of this work has been asserted by him in accordance with sections 77 and 78 of the Copyright, Designs and Patents Act 1988.

The Open Access version of this book, available at www.taylorfrancis .com, has been made available under a Creative Commons AttributionNon Commercial-No Derivatives 4.0 license. No part of this book may be reprinted or reproduced or utilised in any form or by any electronic, mechanical, or other means, now known or hereafter invented, including photocopying and recording, or in any information storage or retrieval system, without permission in writing from the publishers.

Trademark notice: Product or corporate names may be trademarks or registered trademarks, and are used only for identification and explanation without intent to infringe.

British Library Cataloguing-in-Publication Data

A catalogue record for this book is available from the British Library

Library of Congress Cataloging-in-Publication Data

Names: Hyysalo, Sampsa, 1974- author.

Title: Citizen activities in energy transition: user innovation, new communities, and the shaping of a sustainable future / Sampsa Hyysalo.

Description: Milton Park, Abingdon, Oxon; New York, NY: Routledge, 2021. Includes bibliographical references and index.

Identifiers: LCCN 2020055329 (print) | LCCN 2020055330 (ebook)

Subjects: LCSH: Renewable energy sources-Technological innovations-

Research. | Energy conservation-Citizen participation-Research. |

Energy industries-Consumer behavior. | Sociotechnical systems. |

Information society. | Energy policy.

Classification: LCC TJ811 .H988 2021 (print) | LCC TJ811 (ebook) |

DDC 333.79/4-dc23

LC record available at https://lccn.loc.gov/2020055329

LC ebook record available at https://lccn.loc.gov/2020055330

ISBN: 978-0-367-64013-2 (hbk)

ISBN: 978-0-367-68025-1 (pbk)

ISBN: 978-1-003-13391-9 (ebk)

DOI: $10.4324 / 9781003133919$

Typeset in Bembo

by Deanta Global Publishing Services, Chennai, India 


\section{Contents}

List of figures viii

List of tables $\quad \mathrm{x}$

Author biography xi

Acknowledgments xii

1 Introduction: Citizens in energy innovation and sociotechnical change 1

2 The biographies of artifacts and practices methodology for the study of sociotechnical change

3 Initial focus: User innovation in sustainable energy

technologies

4 Broadening the inquiry: New Internet-based energy communities

5 Zooming out: User activities and the series of configurational movements in energy transition

SAMPSA HYYSALO AND JOUNI JUNTUNEN

6 Conclusions and implications for management and policy

Appendix 1: Data and methods on renewables innovation and adoption in the Finnish energy system 


\section{Figures}

2.1 Coverage between the analysis unit, observation unit, and data in technology adoption research

2.2 The analysis units and observation units in more in-depth studies of innovation and organization

2.3 BOAP investigation designs several lines of sub-study of different aspects of sociotechnical change

3.1 Examples of user designs in S-RET systems 38

3.2 The diffusion paths of consumer innovations in S-RETs 51

3.3 A schematic illustration of design spaces under conditions of high variation in user needs

4.1 An entry post-guiding ASHP selection (text translated from Finnish)

4.2 An example of a forum user giving advice for others regarding scaling and selecting a heat pump, and finding a location for it (translated from Finnish)

4.3 Users share heat-pump monitoring data graphs via forums and their own internet sites

4.4 The start of an exchange between members regarding a potential hack

4.5 Cumulative installations and user activity development in the main forum for heat pumps in Finland

4.6 Mapping energy communities regarding the outcome and participation in a digitalized world

5.1 The cumulative number of heat pumps in use during the take-off and acceleration phases of transition

5.2 The actor-level relationships of user activities in Finnish heat-pump transition

5.3 User activities presented at the transition process level 
5.4 A configurational model of technology proliferation in follower contexts

A.1 The sequential mixed-method research design used to study user innovations

A.2 Criteria for user innovations 


\section{Tables}

3.1 User inventions in ASHPs 39

3.2 User inventions in GSHPs 42

3.3 User inventions in wood pellet systems 44

3.4 Expert evaluations of the inventions' inventiveness, ease of implementation, diffusion potential, and energy-saving potential 47

3.5 The diffusion of consumer innovations when innovative peer diffusion is included

3.6 The measures of the association between independent and dependent variables through Fisher's exact test 54

3.7 Mann-Whitney U test results for straight transfer peer diffusion $\quad 55$

3.8 Mann-Whitney $U$ test results for innovative peer diffusion 55

4.1 How the heat-pump forum supports heat-pump acquisition, use and user invention $\quad 76$

4.2 The ecology of Intermediaries in the Finnish heat-pump market in the period 2010-2014

4.3 Case examples of community energy: a local community energy project and a dispersed structure community 


\section{Author biography}

Sampsa Hyysalo is Professor of Co-Design at the Aalto University School of Art, Design and Architecture in Helsinki, Finland. His research focuses on designer-user relations in sociotechnical change. This includes engagement in participatory design, co-design, open and user innovation, open design, peer knowledge creation, user communities, citizen science and user knowledge in organizations. His research orientation is multidisciplinary with science and technology studies, innovation studies, and collaborative design being his main fields. He has authored several books, the latest being The New Production of Users: Changing Innovation Communities and Involvement Strategies (written with Elgaard Jensen and Oudshoorn, published by Routledge, 2016), which won the European Association for the Study of Science and Technology's Freeman Award (https://easst.net/articl e/the-easst-awards-2016), and Health Technology Development and Use: From Practice-Bound Imagination to Evolving Impacts (Routledge, 2010). Sampsa has published over 70 full-length articles and book chapters, including over ten publications in field-leading journals such as Research Policy, MIS Quarterly, Design Studies, and Social Studies of Science. He was the Chief Editor of Science $\&$ Technology Studies journal 2007-2016 and was awarded the Academy of Finland Award for Social Impact in 2010.

\section{Contributor biography, Chapter 5}

Jouni K. Juntunen is a Professor of Practice of Sustainable Production and Consumption with a joint appointment at the Aalto University School of Business (Department of Management Studies) and the School of Arts, Design, and Architecture (Department of Design). His research and teaching focuses on crowd-based organizing in the development of sustainability innovations. He has published in several leading journals such as the Journal of International Business Studies, Research Policy, Journal of Product Innovation Management, Ecological Economics, Environmental Research Letters, and Journal of Cleaner Production. 


\section{Acknowledgments}

This book is a result of a decade-long research journey into energy systems change. It has been fascinating to dive ever deeper into a new and important domain, while retaining my old angle, that of active citizenship in technological change. My greatest thanks go to coauthors in sub-studies that have paved the way for this book: Jouni Juntunen, Stephanie Freeman, Mikael Johnson, Robin Williams, Neil Pollock, Eva Heiskanen, Mikko Jalas, Raimo Lovio, Ville Lauttamäki, Mari Martiskainen, Maija Mattinen, and Ari Nissinen. I have been equally fortunate to work with a broader set of colleagues on social aspects of energy transition, resulting in several parallel streams of research that have supported the present book Tatu Marttila, Pekka Murto, Karoliina Auvinen, Paula Kivimaa, Wouter Boon, Laurens Klerkx, Armi Temmes, Allu Pyhälammi, Kaisa Matschoss, Sofi Perikangas, and Antti Silvast.

Another parallel community whose work is visible in the book is the users in innovation research group at Aalto University, which I have had the privilege to lead over the years leading up to the publication of this book. Along with many of the already mentioned people Cindy Kohtala, Andrea Botero, Kaisa Savolainen, Pia Hannukainen, Samuli Mäkinen, and Svetlana Usenyuk have provided valuable insights over the years and J. Tuomas Harviainen, Brenda Vertiz, and Nathaly Pinto commented usefully on the penultimate version of the book as did Alin Ake-Kob. Over the years many international colleagues have shaped the ideas leading to the book, particularly conversations with Eric von Hippel, Adrian Smith, James Stewart, and Jack Whalen have helped the ideas and formulations that went into the book.

Chapters 2, 3, and 4 in the book were built on earlier articles and permission to republish previously published materials is acknowledged as follows:

Sections 2.2 and 2.3 in chapter 2 have appeared in: Hyysalo, S., Pollock, N., and Williams, R., (2019a). "Method matters in the social study of technology: Investigating the biographies of artifacts and practices." Science and Technology Studies 32 (3) 2-25; some sections in Chapters 3 and 4, and Figure 3.1: Hyysalo, S., Juntunen, J., and Freeman, S (2013). User innovation in sustainable home energy technologies. Energy Policy55 (2013) 490-500; Figures 4.2 and 4.4: Hyysalo, S., Juntunen, J., and Freeman, S (2013). Internet Forums and the Rise of the Inventive Energy User. Science \& Technology Studies, 26 (1), 
25-51; Sections in Chapter 3, Figures 3.2; Tables 3.4-3.8, Figures in Appendix 1: Hyysalo, S., Johnson, M., and Juntunen, J. K. (2017) The diffusion of consumer innovation in sustainable energy technologies, Journal of Cleaner Production, 162, S70-82; Sections in Chapter 4 and Figures 4.1. 4.3 4.5, and Table 4.2: Hyysalo, S. Juntunen, J.K, Martiskainen, M (2018) Energy Internet forums as acceleration phase transition intermediaries. Research Policy 47 (5) 872-885. Shorter passages not exceeding 800 words have appeared in Hyysalo, S. and Juntunen, J. K. (2018) User Innovation and peer assistance in small scale renewable energy technologies. In Davidsson and Gross (eds) Energy in Society Reader. Oxford University Press; Heiskanen, E., Hyysalo, S., Jalas, M., Juntunen, J. K., and Lovio, R. (2014). User involvement and radical innovation: The case of heat pumps in Finland. In Juninger, S. and Christensen, P. (Eds.). Highways and Byways of Radical Innovation: The Perspective of Design. Kolding Design School: Kolding; and Mattinen, M., Nissinen, A., Hyysalo, S., and Juntunen, J. (2014) Energy use and greenhouse gas emissions of air-source heat pump and innovative ground-source air heat pump in cold climate. Journal of Industrial Ecology.

The open-access book is published under CC BY-NC licence, and funded by the Strategic Research Council of Finland consortium 293405 "Smart Energy Transition: Realizing its potential for sustainable growth for Finland's second century". 


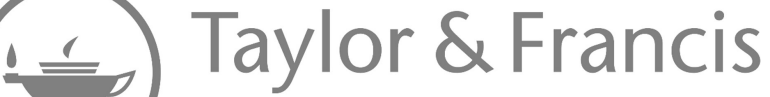

Taylor \& Francis Group

http://taylorandfrancis.com 


\section{Introduction}

\section{Citizens in energy innovation and sociotechnical change}

\subsection{The changing image of energy citizens in sociotechnical change}

Decentralized energy production based on renewable sources is a key change toward fossil-free-energy systems. This change is well underway in many countries, and the replacement of fossil-fuel-based solutions is increasingly taking place in the arena of mainstream deployment and adoption of renewable energy technologies. This means the "demand side" of the market plays a key role in the financing of change as well as in accepting changed consumption patterns and new technologies (IEA, 2018; IPCC, 2018). To many, this sounds a lot to ask given that present energy consumption is among the most passive and routinized forms of consumption there is.

Yet people on the demand side have in many countries taken the initiative (e.g., Ornetzeder and Rohracher, 2013; DeWald and Truffer, 2012; Nielsen, 2016), and when they do so they can do much more than just adopt and finance new solutions. Demand-side activities related to technological innovation, adaptation, new practices, and market creation have been found to be key "enablers" for the proliferation, further development, and mass-market uptake of low-carbon technologies, particularly regarding small-scale renewables such as heat pumps, pellet-burning systems, solar PV, and solar heat, which we examine in this book (Rohracher, 2003; Caird and Roy, 2008; Heiskanen et al., 2010; Nielsen, Reisch, and Thøgersen, 2016; Nyborg and Røpke, 2015; Ornetzeder and Rohracher, 2006, 2013). These findings and their importance run contrary to the mainstream climate and energy policies that have primarily targeted the "supply side" through subsidies, regulation, and supply-end investments (IEA, 2018; IPCC, 2018) and have sought to overcome "consumer barriers" using campaigns, means, and measures intended to better diffuse industry-developed products and improve their use (Mignon and Bergek, 2016; Nye, Whitmarsh, and Foxon, 2010). Consequently, there is a strong and growing interest to better understand citizens, as users, consumers, and civilsociety actors, in energy innovation and transition processes (see, e.g., Smith, 2012; Ornetzeder and Rohracher, 2013; Schot et al., 2016; Meelen et al., 2019; Rohracher and Köhler, 2019).

DOI: $10.4324 / 9781003133919$ 


\section{Introduction}

This shift toward active citizen contributions in sociotechnical change resonates with wider research on users and consumers outside sustainability-related fields. Users were long seen as unlikely agents of technological change (Oudshoorn and Pinch, 2003). For instance, von Hippel and his colleagues have fought a 40-year uphill battle to establish the extent, depth, and significance of user innovation against incredulity from the prevailing innovation studies and policy paradigms that assumed producers and research institutes to be the only significant driving force of innovation (von Hippel, 1988, 2005, 2016). A similar gradual revelation has happened across neighboring disciplines and related topics regarding user participation in design (e.g., Schuler and Namioka, 1993; Voss et al., 2009a, 2009b; Jensen, 2012; Hyysalo et al., 2016a, 2016b), the active consumption of goods (e.g., Appadurai, 1986; Miller and Slater, 2007), and user alterations and redesigns in IT systems (DeSanctis and Poole, 1994; McLaughlin et al., 1999; Kohtala et al., 2020) and their roles in affecting broader sociotechnical change (Fischer, 1992; Kline and Pinch, 1996; Oudshoorn and Pinch, 2003; Williams et al., 2005; Hyysalo et al., 2016a).

A root cause for the long neglect of citizens in innovation and the gradual realization of their importance lies in the invisibility of most citizen contributions to sociotechnical change. Much of this invisibility can be described in terms of being invisible work in three senses: not being recognized as happening or being of importance, not being recorded and thus gradually lost to memory, and not showing up using the typical research instruments used by people studying the area (Strauss, 1993; Strauss and Star, 1999; Verheig et al., 2016). These forms of invisibility have entailed a need to develop specific and often rather arduous research designs in order to make the contributions visible (Suchman, 1995; Szymanski and Whalen, 2011; De Jong et al., 2015; Verheig et al., 2016). But these more detailed studies, in turn, are subject to counter arguments regarding over generalizations made on the basis a few, potentially exceptional, cases and sites. As a consequence, a considerable space for assumptions remains in regard to users' contributions and their importance to innovation and sociotechnical change. Given how hard it is to specifically identify the various forms of active citizen engagement in sociotechnical change, should the found instances be interpreted as being but the tip of the iceberg of a much more varied and plentiful contribution? Or is it more prudent and sounder to assume that the instances found may, in fact, be all there is?

The present book seeks to move research beyond making circles in this assumption space regarding citizens in energy innovation and transitions. As in many other fields, there are some exemplary works on particular citizen activities, such as on innovation (e.g., Rohracher, 2003; Ornetzeder and Rohracher, 2006, 2013; Truffer, 2003; Nielsen, 2016), on consumption (e.g., Nyborg, 2015; Juntunen, 2014a; Palm and Derby, 2014), on social movements (Smith et al., 2014; Hess, 2016; Kohtala, 2017), and on communities (e.g., Heiskanen, johnson et al., 2010, 2015; Smith et al., 2016b). Yet, to date, they have only been connected by summative literature reviews (Smith, 2012; Durrant, 2014; Schot et al., 2016) and arguments made via non-sustainability-related historical 
studies (Kanger and Schot, 2016; Kanger et al., 2018). The detailed evidence is also almost solely focused on the early phases of sociotechnical change and does not properly address the ongoing mass take-up.

The contribution of this book is thus to provide the first detailed, empirically grounded analyses of the activities that citizens engage in concerning sustainable energy innovation, community creation, and sustainability transitions and from the early stages of technological change to mainstream adoption. Methodologically, it elaborates the mid-range research design, the biography of practices and artifacts (BOAP) methodology, that can help achieve such analyses and further elaborate the import of the findings at a theoretical level through conceptualizing sociotechnical change as series of configurational movements that build on each other but change the character of technology and its userships while doing so.

\subsection{Citizens in sustainable sociotechnical change-a short history of reconsiderations}

The terms by which citizens' involvement in sociotechnical change have been addressed vary in different disciplines between user, consumer, customer, and citizen. Marketing and management typically talk of consumers or customers to emphasize the financial relations involved (e.g., Marchand, 1998; Prahalad and Ramasvamy, 2004). Design and computer sciences as well as innovation studies address the topic with the register of "user" that emphasizes the realized use and immediate benefits gained with it (von Hippel, 2005; Hyysalo et al., 2016), while social and political sciences tend to talk of citizens and consumers (Smith, 2012; Voß and Amelung, 2016; Hyysalo et al., 2016). The common denominator in these terminologies is that they denote people whose orientation to technologies is that of first-hand benefit or harm, or who become otherwise directly impacted by them. This is the functional opposite to producers and professional designers who engage in technological change primarily for economic gains made through sales (von Hippel, 2005, 2016; Bjerkness, 1987; Prahalad and Ramasvamy, 2004). Because of this, the present book uses the terms user, citizen, and consumer as partial synonyms and follows the nomenclature used in the literature that is being discussed, most commonly the "user" (unless there is a specific reason to stress one of the other terms specifically, such as when opposing consumers to organizational users or when emphasizing citizenship rights against mere using).

Historically, all the way up to the 1980 s, users were largely seen as insignificant actors in technological change, and findings related to their contributions to innovation, design, and consumption were mostly regarded as idiosyncratic exceptions (e.g., von Hippel, 1976; Rosenberg, 1982; Bjerkness, 1987; Schwartz-Cowan, 1983). Research on technological change and innovation has since acknowledged the role of users but only gradually duly addressed it and discovered the range and extent it may have. This has taken place in several parallel disciplinary streams (for overviews, see Williams et al., 2005; von 
Hippel, 2016; Hyysalo et al., 2016b), which have also gradually found their way into the studies of sustainable innovation and sustainable change.

By the 1990s, innovation scholars and sociologists of technology had recognized the importance of users (von Hippel, 1988; Klein and Rosenberg, 1986; Bieker et al., 1987), yet asserted that if users and other stakeholders have an impact on technology, they must have it before the form and meaning of the technology stabilize (Bijker, 1995; Noble, 1984). Once the technology's design and related infrastructure became locked in place, users' choices appeared to narrow down to adoption or non-adoption (Russell and Williams, 2002). Even within such constraints, users were observed to be active in several important ways, which can be characterized as users having importance in early-stage innovation and shaping of technology, often through civil-society activism and local communities:

a) Users are the sources of inventive new technologies in areas where the available products do not cater to their specific needs. Even though further development often takes place in R\&D companies, the lead-user designs spur on new product lines and improve earlier ones (von Hippel, $1976,1988)$. In renewable energy, this has been found to be the case in wind turbines (Karnøe and Garud, 2012; Nielsen, 2016) and solar collectors (Ornetzeder and Rohracher, 2006).

b) Users have been a vital source of information for developing new design versions as respondents to marketing research ever since the 1930s (Marchand, 1998). In sustainable energy this has been the case with passive houses (Rohracher, 2003; Ornetzeder and Rohracher, 2013).

c) Users can act as design partners, as experts on their own work, and as design decision makers regarding the conditions of their everyday life (Bjerkness, 1987; Bødger et al., 2004). In small-scale renewable energy technology (S-RET), some evidence of this can be found in grassroots innovation (Smith et al., 2016a, 2016b; De Vries et al., 2016).

d) Users also improve early designs through learning-by-using, both locally and through feedback to producers (Rosenberg, 1979, 1982; Lundvall, 1988; Lundvall and Vinding, 2005). In sustainable energy innovation this has been the case with solar heaters and woodchip burners (Ornetzeder and Rohracher, 2006).

e) Early adopters have a strong influence on what direction uses of new technologies and social organization around them take, as well as on the norms governing them. This can affect potential alternative technologies and technology-related citizen activism as well as early phases of mainstream technologies (see, e.g., Bijker, 1995; Fleck, 1993a, 1993b; Flichy, 2007; Akera, 2001; Schwartz-Cowan, 1983; Fisher, 1992). In renewable energy such influence can be observed in the community energy movement as an alternative technological discourse (Hargrieves et al., 2013; Smith et al., 2016b), as well as in how user experiments gradually led to the mainstreaming of wind turbines in Denmark (Ornetzeder and Rohracher, 2013; Nielsen, 2016). 
f) Users can also actively oppose or passively reject new technologies, or they can undermine their intended effects by failing to use them according to expectations (Akrich, 1992; DeSanctis and Poole, 1994). In S-RET this has been observed with added heating and cooling to passive house concepts in many countries (Ornetzeder and Rohracher, 2003; Palm and Derby, 2014).

The next set of reconsiderations emerged over the course of the 1990s, when the emergence of more flexible home and workplace information technologies made researchers realize that users alter and adjust technology-in-use more than had been assumed. The emerging more open and flexible development processes did not result in a similarly "closed" technology, as had been the case in the earlier mass-manufacturing era. The most important findings can be characterized as recognizing the commonness of the active shaping of technology-inuse and cyclical development of technology:

g) Studies of home consumption revealed that instead of being passive adopters, ordinary consumers were active in adapting the configuration and meaning of the technologies to make them work (Silverstone et al., 1992; Lie and Sørensen, 1996). They were, by default, domesticating technology into the moral economy of the household and contributing to the long-term taming of new types of technology. Further research has since shown this to take place beyond ICTs (Miller and Slater, 2007; Berger et al., 2006). There is some evidence of the active domestication of sustainable energy solutions and even "domestication pathways" from one S-RET to another (Palm and Derby, 2014; Juntunen, 2014; Nyborg, 2015).

h) Studies of workplace information systems showed that selective appropriation, integration into other devices, the co-evolution of practices and new technology, add-on solutions, new uses, (re-)inventions, and efforts to market the technology were, in fact, very common (see, e.g., DeSanctis and Poole, 1994; Alter, 2006; Szymanski and Whalen, 2011; McLaughlin et al., 1999). In sustainable energy such adaptation has been documented to some extent prior to the present line of study (Heiskanen et al., 2015; Raven et al., 2008; see Chapters 3 and 4).

i) Home multimedia and workplace ICTs showed how more advanced peers, "warm experts," were central in educating other users (Bakardjieva, 2005), as were semi-professional "local experts" who seconded their help in addition to their main jobs (Stewart, 2007; Voss et al., 2009), becoming "user-side innovation intermediaries" (Stewart and Hyysalo, 2008). In sustainable energy, user-side intermediation has been documented but seldom conceptualized thoroughly (Heiskanen, johnson, et al., 2010; Raven et al., 2008, 2015; deVries et al., 2016; Meelen et al., 2019).

j) It was further realized that many new technologies did not follow linear patterns where the design becomes "closed" before it starts to diffuse 


\section{Introduction}

(Fleck, 1993a; Williams et al., 2005) but involved innofusion, that is, iterative loops between design and use, often lasting as long as several product generations (Pollock and Williams, 2008; Hyysalo, 2010). Users' domestication and alterations can thus shape the technology, both at local adopter sites and through entering the many feedback loops that circle between suppliers and users. In sustainable innovation this has been discussed by Heiskanen et al. (2014) and under the headings of learning-byusing (Ornetzeder and Rohracher, 2013) and user assemblages (Nielsen, 2016).

The third and most recent reconfiguration in the understanding of the role of users in technological change has resulted in the rapid proliferation and sophistication of digital-sharing platforms throughout the 2000s and 2010s. The ensuing changes can be characterized as user contributions becoming boosted through new digitally connected peer communities:

k) Previously unconnected users have formed communities of interest on the web that share and iterate designs. Such user-innovation communities have proliferated far beyond open-source software and are designing many products without suppliers (Tapscot and Williams, 2008; Jeppesen and Molin, 2003; von Hippel, 2016). In sustainable energy this has not been documented prior to the present line of study (see Chapter 4), but after our research others have identified it as well (Meelen et al., 2019).

1) Manufacturers, in turn, are busy setting up their own user-innovation community efforts (Jeppesen and Fredriksen, 2006; Fuller, 2006; Pollock and Hyysalo, 2014). Living labs, web-based innovation areas, and user groups mark some of the widespread practices through which users are actively connected to each other and to producers in order to facilitate company research and development activities (Johnson et al., 2014; Leminen, 2015; Hyysalo and Hakkarainen, 2014; Mozaffar, 2016). This has not been reported in sustainable energy innovation to date, but is likely to emerge.

m) User-configurable content and derivative designs have become more commonplace, particularly in social media applications, games, and masscustomized products (Benkler, 2006; Tapscot and Williams, 2008; Botero et al., 2010). In sustainable energy this has not been documented prior to the present line of study (see Chapter 3).

n) Internet user forums, blogs, and discussion platforms have allowed users to pool their experiences and reveal their designs to other users. This has led to a "do-it-yourself renaissance," in which self-created and collectively created artifacts are gaining new impetus (see, e.g., Kuznezov and Paulos, 2010; Grabher and Ibert, 2014; Kohtala, 2017; Kohtala et al. 2020). In sustainable energy this has not been documented prior to the present line of study (see Chapter 3), but since then, it has also been recognized elsewhere (Meelen et al., 2019). 
Taken together, these active roles by users underline that they can be a major contributing force, as well as an inhibiting force, for a given innovation and in long-term sociotechnical change. As can be observed from the above, the research that exists regarding sustainable energy has mostly concentrated on those types of contributions to innovation and sociotechnical change that were already well elaborated in the 1980s and 1990s in the wider academic community. Correspondingly, there is a relative neglect of the new patterns and opportunities that have opened up in this millennium. Also the empirical incidence and interrelations between the different contribution types need further research: do some types feed into others, place conditions, or inhibit others? And if so, by what processes? Thus far studies have targeted different technologies, different aspects, settings, and times in sociotechnical change and thus resulted in rather pathwork understanding of users in sociotechnical change that has high likelihood of biases.

\subsection{A new approach to researching users in sociotechnical change}

A new way to study users in sociotechnical change has been developed in the biographies of artifacts and practices (BOAP) approach (Hyysalo et al., 2019a). The BOAP approach entails a long-term serial ethnographic and historical study of sociotechnical change, in short, providing a mid-range methodology that is at once capable of zooming into activities, such as those by users, that are not flagged prominently in media or existing data sets and zooming out for longitudinal analysis of their importance in sociotechnical change. The methodology is further conducive to empirically grounded theorization and bridging different theoretical positions and empirical findings - particularly temporally and spatially more narrow or hollow depictions as we discuss in chapter 2 (Hyysalo et al., 2019).

In this book, the BOAP approach is developed further in order to address the patchwork and siloed nature of studies of users as in three research communities, those of user innovation, sustainability transitions, and sciences and technology studies (S\&TS), which would all benefit from BOAP studies, albeit for different reasons. The aim of the book is thus as much substantive as it is methodological and aimed toward theorization.

User-innovation research has had pivotal importance in surfacing the amount of innovation made by users, and in doing so, showing how technological change is importantly affected by a range of actors and processes whose contributions remain invisible or shadowed by entrepreneurs and research institutes who (must) seek publicity for their commercial and research activities in order to be able to accomplish them (von Hippel, 2005, 2016). However, user-innovation research has limited itself to the invention and early commercialization phases and has been heavily disposed toward the study of new objects, be these physical or digital. This focus de-emphasizes users' other contributions to (often long and serial) innovation processes-such as new uses, new procedures, partial uses, collaboration with producers, and so 
on-which typically accompany further innovations by users, as well as producers, and is an important dynamic to understand better (cf. e.g., Hienert et al., 2014; Hyysalo, 2009; Kohtala et al., 2020). Going further still, a wider cast of user activities involved in the shaping of sociotechnical change-such as adjustments to daily routines, recommendations, rejections, reclamations, lobbying, community building, and maintenance to mention a few-become cut off from the innovative activities of users. The picture of the forms by which they affect sociotechnical change, including the embedding of their innovative activities, remains incomplete.

The user-innovation researches focus (and its ensuing limits) is not accidental. It results from its orientation to establish the relative incidence of user and producer innovation under different conditions and the preference for variance epistemology and quantitative research designs to argue this case convincingly (von Hippel, 1988, 2005). Variance epistemology has, however, significant shortcomings in the analysis of contingent processes and emergent phenomena (van de Ven et al., 1999; van de Ven and Poole, 2005; Garud and Gehman, 2012), and the present book's approach combines quantitated user-innovation research with detailed qualitative ethnographic work on user activities, as well as with process epistemology, in order to open up new substantive research and theoretical extensions regarding how users contribute to innovation and sociotechnical change.

Sustainability transitions research has recently emerged as a fusion point for understanding sociotechnical change. Its key contribution has been its ability to offer relatively clear starting and ending points for the study of sociotechnical change (Geels, 2002; Köhler et al., 2019). A focus on the sustainability of sociotechnical systems, premised on path dependency and interlocking of system elements, allows transitions research to outline a long-term change from one dynamically stable sociotechnical regime to another via different pathways, such as substitution or reconfiguration (Geels et al., 2016). Systems change typically lasts for decades and it provides a backdrop within which various innovations - as well as user contributions, their interrelations, and their relations to other actors - can be examined. In other words, the idea of transitions promises to provide a vantage point for "full cycle" analyses of how production and consumption interrelations change (Köhler et al., 2019).

However, owing to its roots in rule-governed systems and long time-span analyses (Geels, 2002; Köhler et al., 2019), transitions research tends to employ a distanced analysis of sociotechnical change in order to cover the width and length of the phenomena involved. Similarly to earlier innovation systems research (Lundvall, 1988, 2005), less visible actors and processes-such as users, learning, and knowledge building - are acknowledged as important but then treated in assumption-prone fashion and only at the aggregate level (for critiques, see von Hippel and Tyre, 1995; Tyre and von Hippel, 1997; ScottKemmis and Bell, 2010; Hyysalo, 2009; Miettinen, 2003; Mierlo \& Beers, 2020; van Poeck et al., 2020). Consequently, the research and methodology needed to truly render visible such intra- and inter-actor processes remain decoupled from long-term change analyses. The present book elaborates a 
research approach that can span careful site-specific studies covering long-term change in the sociotechnical change in question; thus, it holds the potential for emending this shortcoming in transition studies.

In contrast, SETS excel in detailed ethnographic accounts of the complex and typically hidden aspects of sociotechnology. Its (material-social) constructivist methodology has been specifically developed for surfacing and accounting for contingencies and the emergent composition of objects, processes, and practices in context-in short, for providing a sophisticated process epistemology for studying sociotechnical change (Bijker, Pinch, and Hughes, 1987; Williams and Edge, 1996; Hess, 1997; Garud and Gehman, 2012). It thus provides resources for addressing the above-noted shortcomings in user-innovation and transitions research on users.

Yet, S\&TS's epistemic anchoring to ethnographically detailed studies of particular sites has tended to limit its yield in relation to characterizing sociotechnical change, both methodologically and theoretically (Hyysalo et al., 2019). Comparison and generalization across the patchwork of different sites, times, peoples, and technologies is a challenge to all innovation and technology analysis, but it is particularly thorny for constructivist S\&TS epistemologies. Leading authors have concluded that S\&TS rather provides generalizability through concepts that capture process patterns rather generalizability across comparable cases (Williams and Sørensen, 2002). The BOAP framework developed at the intersection of S\&TS and innovation studies is a response to the quandary between the requisite specificity of studies and improved generalization. What is at stake is not pining back to objectivistic generalization but being able to better clarify how different sites, times and types of shaping technology matter in relation to each other.

Reflecting on these methodological underpinnings of the volume, Chapter 2 is devoted to expanding on the BOAP research approach. Regarding the ensuing theorization on sociotechnical change as series of configurational movements many of which involve users (as we discuss in chapters five and six), the focus on sustainable energy technologies is interesting because of the implied societal relevance and import to the everyday lives of large amounts of people. S-RETs are serious, even somewhat boring technologies that hold important capacity in relation to addressing climate change. These are not esoteric technologies - such as scientific instruments (Riggs and von Hippel, 1988) and rugged terrain vehicles (Hyysalo and Usenyuk, 2015). Neither are they mere playthings or sports equipment — such as windsurfing and kitesurfing equipment, mountain bikes (Lüthje, 2004; Lüthje et al., 2005) and rodeo kayaks (Hienert et al., 2014) - in which users have been documented to be in the driver's seat of technological change. Nor are they digital-only technologies that arguably have different patterns of change than their physical counterparts (Baldwin \& von Hippel, 2011; Benkler, 2006). Thus as 'models organisms' for better understanding sociotechnical change S-RETs present different, and perhaps more convincing starting point, than many domains in which users have hitherto been shown to be of pivotal importance. 


\subsection{An overview of the volume}

\subsubsection{Chapter 2, "Biographies of artifacts and practices in sociotechnical change"}

Sophisticated understandings of the technological change produced by innovation studies, transition research, and social studies of technology are, paradoxically, at odds with their own dominant research designs and the methodological guidelines. A key insight from the social shaping of technology research and process studies of innovation has been that new technologies are formed gradually in multiple, particular (albeit interlinked) settings, by many different groups of actors, over long periods of time. Nonetheless, common research designs have not kept pace with these advances in knowledge. The mainstream innovation studies continue to treat sociotechnical change through quantitated data series and statistical analyses (variance epistemology), which freezes the identity of each innovation and actors and organizations involved to just one point in time, while it is well known that all these tend to change in the course of innovation projects. Alternatives developed to better address the processual nature of change have resorted to intensive ethnographic engagements on particular sites or broad-stroke historical studies and systems analyses, yet have been unable to address both the intricacy of sociotechnical phenomena and the extent of the shaping process in tandem. To ensure that research is able to continue to provide grounded insight into the processes of innovation and sociotechnical change, this book argues for the ambitious reconceptualization of research designs. This would include a move from simple "snapshot" studies and broad-stroke "hollow" evidence to long-term research designs that progress through a weaving together studies in different settings, at different times, and with different granularities of analysis.

There has consequently been increasing interest in extending current methodological and analytical approaches through longitudinal and multi-site research templates. The BOAP framework deployed and taken forward in the present book builds on a 20 -year-long body of studies in different sociotechnical settings. Chapter 2 outlines the rationale and basic principles of BOAP. It elaborates how they differ from more conventional methodological approaches in studies of innovation and diffusion through a detailed discussion of the interrelations between observation units, analysis units, and research questions. The chapter further elaborates the data and analysis methods in the BOAP study underlying the present book and elaborates its theory-building approach, which differentiates the methodology from naively inductive or deductive research orientations and methodology templates.

\subsubsection{Chapter 3, "Initial focus: user innovation in sustainable home energy technologies"}

The new millennium has seen an increasing interest in citizens as energy endusers. While much hope has been placed in more active energy users, it has 
remained less clear what citizens are able and willing to do in shaping new technology. An investigation into inventions and modifications to S-RET conducted in Finland revealed a surprisingly high amount and depth of innovative activity. Over 200 inventions or modifications by consumers have been made to S-RETs, such as heat-pump, solar, and wood pellet-burning technologies. These inventions improved either the efficiency, suitability, usability, maintenance, or price of the S-RET systems. The analysis further clarifies that users are able to successfully modify, improve, and redesign almost all the subsystems in these technologies. The rationale for user inventions is based on the fact that supplier models do not sufficiently cater for the geographic location and house-specific variation in weather, material, aesthetic, and regulatory conditions, which leaves the local design space for users to improve their S-RET systems unexplored. In addition to expert evaluations, life-cycle carbon assessment was performed on selected user inventions, verifying their positive climate contribution.

The innovation activities of consumers support the proliferation of sustainable energy technology in contexts where institutional and technology characteristics are not yet fully developed for the wide proliferation of S-RET. Further analysis focused on how consumer-created technology solutions diffused. Their existence adds to solution variety, but do others adopt their solutions? The findings show that $2.7 \%$ of the consumer innovations diffused through commercial channels and $8.2 \%$ diffused through their straight adoption by peers. A significant share of projects (34.1\%) were, however, part of "innovative peer diffusion" adoption that included adaptations and further modifications carried out among peers. Innovative consumers' efforts to diffuse their solutions remain at a low level and indicate directions for platform development, by which these solutions may spread more widely. Taken together, the analyses counteract the false assumption that users can only make low-tech add-ons, as well as the equally untenable hope that citizen users could take over the design of renewables altogether if only given the chance and incentivized properly.

The actions of a typically small group of inventive users are embedded in and supported by the activities of a broader user base in internet communities, and it may be that the greatest importance of user innovators does not lie in the innovations themselves but in the sharing of competence that these inventive users do among peers (this topic is interrogated further in Chapters 4 and 5).

\subsubsection{Chapter 4, "Broadening the inquiry: new internet-based user communities"}

Users' capacity to carry out their inventions (described in Chapter 3) owes much to their exchanges on user-run internet communities, a new and proliferating type of setting. The broadening of the focus to cover these novel internet communities is done in Chapter 4, pursued through ethnographic studies on the internet and in users' homes. In internet forums, people organize 


\section{Introduction}

themselves around products and technologies in order to discuss use, purchasing, experiences, and community knowledge about products and producers (and so on). These online forums help otherwise dispersed and heterogeneous users to create a specific kind of learning environment that helps people to grow more knowledgeable of the technologies in question. The learning is carried furthest by inventive users, who also give back to these internet communities by providing deep-end peer support for other users.

From a systems change perspective, internet communities support the adoption and diffusion of S-RET. While internet communities are common far beyond energy technologies - their different forms can be found related to almost every serious technology and hobby area-they have become significant in S-RET diffusion and related systems changes in many countries and for many S-RET technologies. Their significance lies in that they qualify market information, articulate demand, and help citizen users to reconfigure the standard technology in order to meet the specificities of different local contexts. Such user intermediation and market creation activities are important in expanding the markets for S-RET beyond enthusiasts, environmentalists, and other early adopters. The early majority of adopters demand more exposure, clearer information, and less uncertainty about new technology options. The forums make the co-existence and interchange between different peoples, competences, and interests possible, which in turn becomes key in meeting the variety of market, institutional, cultural, and environmental conditions which S-RET faces in different contexts.

These internet energy communities are a new type of energy community that differs from the traditional local community energy that is premised on shared ownership and output, as well as distributed energy cooperatives. The nature of internet communities is that they are dispersed, and they do not center on shared produce but on sharing information, knowledge, and procedural advice. The dispersion over the internet allows these communities to bridge much wider geographical distances and their mass of participants allows for a plurality of orientations to coexist-offering a critical mass of competencies with which most problems can become clarified if not solved. These facets allow the new internet-based energy communities to form a "boundary infrastructure" among users that can perform the knowledge-related facets of energy communities more effectively and widely than the locality-based traditional forms. This also affects the nature of the discourse in the forums; instead of the critical and empowering energy autonomy-related discourse found commonly in energy communities, the discourse in (the successful) internet-based energy communities tends to foster what could be called "appreciatively critical" discourse on the focal technology. This discourse purposefully downplays general technology critique and ideological underpinnings in favor of naturalizing discourse, which opens the technical black box of technology and market functioning, but contains the scope of alternatives. This type of discourse appears to be rather effective in increasing the legitimacy of the focal technology in society. 


\subsubsection{Chapter 5, "Zooming out: user activities and the series of configurational movements in energy transition"}

The chapter zooms out from the detailed studies of S-RET innovation and adoption and provides a longitudinal analysis of user activities in the proliferation of heat pumps in Finland. It does so through the BOAP approach and presents a new type of analysis in transition studies S\&TS. The ethnographically detailed and historically extended longitudinal investigation on heat pumps in Finland spans from the beginning of the transition to the late acceleration phase, a considerably longer span than the previously detailed analyses on users in transitions.

The analysis shows that users have been active in adaptation and adjustment, user innovation, championing projects, forming user communities, user-side intermediation, market formation, and legitimacy building. These user activities primarily feed into each other and spur on the diffusion of S-RET. While most of the user activities shape the expanding technological niche to some extent, in particular, the increased adoption rates also affect other actors in the expanding niche, as well as related regime actors and the broader heating system landscape in a particular country setting.

The analysis in Chapter 5 questions the theory- and literature-review-based assumptions about particular user activities being associated with specific transition phases. Most user activities take place from the early upscaling phase to the late acceleration phase. While intermediation, market creation, and legitimacy building intensify from the take-off and early acceleration phase of transition onwards, this is particularly thanks to the emergence of the internet communities discussed in Chapter 4. Assertions of the relative importance or incidence of different user activities beyond this would not be warranted as their relative incidence, let alone importance, becomes next to impossible to study reliably.

The chapter further theorizes that the development and use of S-RET in each new country context should not be seen as a linear diffusion process or even a quasi-linear social-embedding process; it should be seen as an extended innofusion process, wherein technology, market, technology use, and governance characteristics evolve in tandem, typically riddled by an uneven pace of advancement and facing various hindrances. The importance of the innofusion view of transitions is underpinned by the fact that most transition to sustainable technologies in most countries and regions are, necessarily, early or later followers, and not taking place in globally early settings. Ironically, the dominant transition models are anchored to broad-stroke histories and detailed studies of globally early settings in sustainable change, which are in fact be rather poor "model organisms" for understanding how the majority of transitions play out on the ground in specific countries, areas, and locales. The analysis of the early-follower country context of Finnish heat pumps opens questions as to the adequacy of transition models for the logical majority of settings. 


\section{Introduction}

\subsubsection{Chapter 6, "Conclusions and implications for management and policy"}

The concluding chapter spells out the overall importance of the analyses to the three theory traditions motivating the book, namely innovation studies, transition studies, and S\&TS. The importance of users in sociotechnical change is not limited to just providing variety in technological solutions at the onset of sociotechnical change, to then be exploited or discarded by the market as a selection environment. Instead, we see continued user-innovation and peer assistance that goes into tailoring the technology to new settings in which it becomes gradually adopted. The influence of users reaches beyond inventions into competence building and peer-to-peer interaction, providing wide support for adoption and adaptation and other user intermediation, legitimacy building, and market creation.

Internet communities allow for the plurality of user orientations to come together and feed into each other. They provide a wide reach that is independent of physical locality and access-time coordination: it is these capacities that render them effective new energy community forms. This stands in contrast to community energy and innovation communities that are unlikely to grow into substantial forces for multifaceted sociotechnical change unless they grow into diverse and trans-local coordinated movements. Their limitations for doing so have to do with the confines set by space and uniformity of orientations and competencies with respect to "serving" tens of thousands of diverse peers.

Overall the importance of users in sociotechnical change is best theorized as being comprised of a series of configurational movements that gradually congeal sociotechnical relations into increasingly stable objects and market relations. Such an extended innofusion process provides an alternative account of sociotechnical change to that of social-embedding and generic-transition models that have assumed relatively stable technology characteristics and adopter identities after the early pre-development phase.

The concluding chapter further elaborates what it would mean to move to more user-driven energy business and energy policy. The current actions energy companies and energy technology companies flag as "customer-centered" and "user-driven" mostly just tune their offerings to the customers in a manner that has long been regarded as business-as-usual in most other sectors such as ICT. Deeper user orientation would follow from business and policy geared to work in partnership with consumers in realizing more ambitious lowcarbon solutions and yet deeper user-driven orientation would depart from the actions already taken by consumers to develop radical business and policy actions. 


\section{The biographies of artifacts and practices methodology for the study of sociotechnical change}

\subsection{Introduction: facing the inconvenience of sociotechnical change in social analysis ${ }^{1}$}

A considerable part of sociotechnical change is inconveniently structured for social scientists. The settings and practices that shape technology are complex and intricate, making them laborious to investigate compared to more commonplace areas of social inquiry, such as studying interactions, attitudes, or institutions and organizations. This has pushed many scholars of the social study of technology toward intensive ethnographic engagements with particular contexts. This particularly concerns the many types of user contributions to sociotechnical change outlined in the introductory chapter, as they tend to fall within the expanse of the invisible work related to science and technology making (Strauss and Star, 1999; Kohtala et al., 2020).

While the intensive ethnographies have proven to be an important strategy for an adequate understanding of sociotechnical change, it has been recognized that many processes of sociotechnical change occur over years and decades and across a range of contexts. New technologies are shaped in multiple interlinked settings and processes characterized by high contingencies and many different choices. The analytical templates needed to cover such broad expanses of time and space have, by default, resorted to coarser "granular" analysis and description. This means that the study set-up-from the outset-brackets out the underlying complexity and intricacy of phenomena. The net result has been the "hollow" or "smoothed" description and analysis of phenomena: a complex phenomenon is noted and may even be used as an explanatory factor, but its actual realization or the dynamics within it are not attended to adequately (Miettinen, 2003; Hyysalo, 2009; Scott-Kemmis and Bell, 2010).

These two key insights - the need for intensity and the expansion of analysis-emerged as early as the 1980s and 1990s in studies of the social studies of technology (SST) through the separate research programs of ethnographers, sociologists, and historians of technology (McKenzie and Wajcman, 1998; Williams and Edge, 1996). Yet their combined effect continues to hold an inconvenient methodological truth for the social study of technology: to account for the shaping of technology, one would need to study the wide

DOI: $10.4324 / 9781003133919$ 
range of settings and the interlinkages between them, as well as the intricate practices therein (Williams and Edge, 1996). It would not be enough to posit that a framework would need to study both actors and structures, and stability and change (e.g., Bijker, 1995; Geels et al., 2016); one must also study these and their interrelations together.

The inconvenience is that realizing the above ambition is much easier said than done. The early technology studies' understanding of sociotechnical change emerged from a patchwork of ethnographic and historical studies that either focused deeply on one or a few moments and sites or, alternatively, sought a broad-brush overview of technology development with a few illustrative deeper examples added (for more detailed critique, see Russell and Williams, 2002; Hyysalo, 2010). This inconvenience became further aggravated in the 2000 s when the "mark 2" social shaping of technology studies discovered the importance of technology-in-use (see Chapter 1) and the importance of social movements and other stakeholder groups in the shaping of technology (Sørensen and Williams, 2002; Williams et al., 2005), thus adding to the array of relevant settings to be studied in-depth and then connected in order to produce an understanding of the dynamics of technological change (Pollock and Williams, 2008, 2016; Stewart and Hyysalo, 2008).

\subsection{Framing effects and a premature sense of closure}

The result was a methodological paradox. SST had produced an understanding of technological change that was arguably more sophisticated and grounded than that provided by mainstream innovation studies - yet it was one that would require research designs that were at odds with SSTs own study templates.

The gravity of these "framing" or "closure" effects was brought to the fore in the 2000s as researchers sought more complex research designs to remedy the paradox (Stewart and Williams, 2005; Pollock and Williams, 2008; Hyysalo, 2010). These research designs repeatedly indicated that studying any given moment and site of innovation would give a significantly different picture of the agency, structure, impact, and materialities related to the technology under study.

In this light, many fellow researchers, and previous studies by the author as well, have fallen victim to a false sense of "natural closure" when conducting their studies, believing there was little point in extending studies to new settings and levels of analysis. Another way to put this is that a premature sense of theoretical saturation (Glaser and Strauss, 1967) is common when researching sociotechnical phenomena.

From the variety of potential closure effects, the first to gain attention became conceptualized as "snapshot bias" (Pollock and Williams, 2008). For instance, when the design of new technology was studied, users were found to be actively configured (see, e.g., Woolgar, 1991; Oudshoorn et al., 2004). However, when technology was followed into use, the users would, in turn, be found to reconfigure (or domesticate) both the technology and the designers (Mackay et al., 
2000). Further on, episodes of the co-configuration of technologies, designers, and users were discovered once the research design was extended to cover these events (Hyysalo, 2004, 2010; Pollock and Williams, 2008). In all, such studies comprised an expanded approach to study the "biographies of artifacts" and their varying shapes (Kopytoff, 1986) across a usually great variety of settings and temporal time frames. In other words, we find that not only are methods performative (Law, 2007) but core issues in research design, such as choosing the site and time of the study, could prefigure which kind of sociotechnical shaping processes one would be disposed to find and, importantly, which ones one would be very unlikely to encounter (Hyysalo, 2010).

The second framing effect concerned the granularity of data and analysis (Hyysalo, 2010) and is in this book is called hollow-arch bias. We define hollowarch bias as a portrayal of a sociotechnical phenomenon with a high level of abstraction that neglects some of the underlying mechanisms that question or invalidate parts of the high-level construction. The constructs appear solid until one digs beneath their empirical surface.

Hollow arch does not mean "a house built on sand" or "a house of cards," as in having no empirical backing for theory constructs at all-there is some backing, yet it is of a limited and superficial kind. And this is part of the problem: as there is some support, scholars who prefer to operate at the broad systems level continue to take for granted and use the hollow constructs and therefore do not complicate their work with more complex and research-wise more inconvenient findings and theoretical constructs on which more focused studies insist.

A prominent example of a hollow-arch construct is that of post-implementation learning effects, known as learning by doing, using, and interacting (Rosenberg, 1979, 1982; Lundvall, 1988). The continued improvements in manufacturing efficiency (learning by doing), the usage and development of tools (learning-by-using), and interactions between producers and users (learning by interacting) appeared straightforward when changes from one product version to another were compared (e.g., Arrow, 1962; Gardiner and Rothwell, 1985) and then taken as pillars for various innovation system constructs (e.g., Lundvall, 1988; Lundvall \& Vinding, 2005). But once these learning processes were studied in more detail, most of the "learning" in these conceptions turned out not to be learning at all as the outcome changes had resulted from changes that happened during the actions, organizations, interactions, arrangements, designs, and materials used, often without recourse to possible learning at the manufacturing and user sites (von Hippel and Tyre, 1995; Scott-Kemmis and Bell, 2010; Nielsen, 2016; Miettinen, 2003). Just as importantly, when the learning that was taking place was studied in detail, it turned not to be as straightforward as depicted by the learning by doing, using, and interacting model (Lundvall, 1988). Instead, the learning processes and outcomes were found to be haphazard, vulnerable, conflictual, and required high amounts of work to coordinate between parties (Hasu, 2001; Hyysalo and Lehenkari, 2002; Hyysalo, 2006, 2009; Hyysalo and Hakkarainen, 2014). 
The point is this: also in other sociotechnical phenomena, high grain-size materials have a tendency to produce hollow-arch descriptions. They may be amenable to neat explanations while overlooking the details of the underlying phenomena that may substantively invalidate the theory constructs laid on top of them. If, and oftentimes as, the more fine-grained studies do not produce similar aggregate level data than that on which the hollow-arch constructs are built on, the concerns raised remain conveniently ignored. The bias resulting from hollow-arch and snap-shot study templates is thus similar regarding the false sense of adequacy in explaining sociotechnical phenomena, even though the mechanism by which the 'blinkering' occurs is very different: one results from an overemphasis on particular study types and sites, and the other from an over-zealous abstraction.

The knowledge gained from research programs that combined both intensive and expansive studies gradually lead to the articulation of a longitudinal multi-site research approach called the "biographies of artifacts and practices" (BOAP) approach. This perspective has evolved from its onset in the early 2000s to a point today where some 30 long-term studies have been conducted with it.

\subsection{The BOAP approach: key characteristics}

The BOAP approach is a methodological approach to the study of sociotechnical change that-even though it has its roots in research informed by broader theoretical approaches of the social shaping of technology (mark 2), symbolic interactionist S\&TS, and activity theory-has purposefully been partially detached from these theories and rendered compatible with several substantive traditions in the S\&TS field and innovation process studies.

BOAP studies feature eight recurring characteristics, which can be considered core markers of the approach. Most BOAP studies feature a varying subset of these methodological responses to common contingencies and differences in the settings of sociotechnical change, in other words they do not present a formula to be applied in all research. The contingencies of sociotechnical phenomena make it difficult for researchers to reliably predict in advance (for instance, on the basis of theory) what might be revealed and occluded by selecting a singular vantage point or a limited set of vantage points, and each of the BOAP characteristics has arisen to handle this challenge. The common characteristics are as follows:

1. Spatial and temporal reach that is sufficient to empirically engage the dynamics of the studied phenomenon (e.g., studies could look at an individual innovation together with the evolution of an industrial field). BOAP studies encompass multiple loci and times wherein sociotechnical change is shaped and moves beyond singular "snapshot" accounts. This is in line with the ambition of multi-sited ethnography to go beyond particular organizational settings, being particularly relevant to the highly dispersed 
processes of scientific and technological life (Hine, 2007; Monteiro et al., 2013; Silvast and Virtanen, 2019; Marcus, 1995) and by those advocating more structural considerations as part of S\&TS analyses (e.g., Klein and Kleinman, 2002; Russel, 1986; Silvast et al., 2013).

2. The shaping of technology and practices is analyzed as taking place within ecologies of interconnected actors. This means not only studying the actors with respect to how they affect the studied technology (such as in Bijker's [1995] "relevant social groups"), which leaves aside the rationales by which they operate and often also the complex and subtle additional mechanisms by which actors relate (Hyysalo, 2010; Pollock and Williams, 2016). This BOAP premise bears a close similarity to those developed in, for example, "linked ecologies" (Abbott, 2005), the "social worlds/ arenas" framework (Star and Clarke, 2003), and studies of the "networks of activity systems" (Engeström, 2000).

3. It may be particularly fruitful to identify and research interstices, the moments and sites in which the various focal actors in the ecology interlink and affect each other and the evolving technology. An overall understanding of the ecology of actors is typically used to pinpoint key locales where these interstices may be researched in detail. The focus on interstices is shared by many in S\&TS, and the use of broader-scale analysis to identify the sites to focus on is found in studies of infrastructures (Monteiro et al., 2013; Ribes and Polk, 2015) and in studies examining the evolution of scientific fields (e.g., Cambrosio and Keating, 1995; Edwards, 2010; Fujimura, 1996).

4. Research is pursued at multiple temporal and spatial scales. The BOAP studies that are at odds with accounts that assume sociotechnical change could be adequately understood through "bird's-eye" descriptions only. There is a need to bridge the analyst's bird's eye view and the actors' real-time "frog's-eye" perceptions, which typically feature high levels of uncertainty and contingencies (e.g., the "fog of innovation" [Höyssä and Hyysalo, 2009]) that can entirely disappear from historical data and broad overviews. Hollow-arch biases and other data granularity related biases are discovered in BOAP investigations time and again. Questioning the dominant research framings in literature can be the starting point for an inquiry into a richer set of contexts (e.g., Stewart and Hyysalo, 2008) or the major outcome of the investigation (e.g., Hyysalo, 2010; Pollock and Williams, 2008, 2016). Multiple-scale research designs can be found elsewhere as well, for instance, in technology and organization studies, in studies of practices (e.g., Nicolini, 2012), in activity theory (Cole, 1996; Engeström, 2000), and in the symbolic interactionist social worlds/arenas framework (Strauss, 1978; Becker, 1982; Clarke and Star, 2003; Clarke, 2005).

5. Different temporalities and spans of change are seen as multiple enacted contexts (Hyysalo, 2010), not as the ontologically distinct layers that are presumed to exist, for example, in the multi-level perspective (Braudel, 
1995; Geels, 2002; Geels and Schot, 2007) or the traditional sociological approaches to the context that locate action within a context conceived as "surrounding layers" (Strauss and Corbin, 1998). In BOAP, events are seen as simultaneously constituting and being constituted by broader patterns: the context for any situation is understood as being comprised of differently paced constituents, as previously discussed in microhistory (e.g., Levi, 1988) and socio-cultural psychology (Cole, 1996; Engeström, 1987), and in distributed cognition (e.g., through the "Hutchins' cube" where the same moment is analyzed in terms of the development of practitioners, practices, and the situated enactment of action [Hutchins, 1995]). BOAP thus seeks to inquire into the links between relevant constituents in order to see their influences and interrelations (or the lack thereof). Studying different contextual constituents means employing an array of often differing conceptual tools, analysis types, and methods in diverse materials (Hyysalo, 2010: 43). BOAP's preference for ethnographic study thus does not mean an in-built "micro-sociological" focus but an examination of how the structuring elements are present in real-life situations and, in turn, how the situations re-shape the structuring elements and what can be learned about the patterns and structures as they are enacted. The position resonates with Situational Analysis by Clarke (2005), yet refrains from flattening the empirically salient topologies in contextual factors (Star, 1996; Akera, 2007) and thus differs markedly from actor-network theory (Latour, 1987, 2005) or ethnomethodology (e.g., Suchman, 1987).

6. Akin to many STS approaches, BOAP studies insist on paying attention to materiality: the content and form of technology as it shapes, and is shaped by, the interrelations between actors (Latour, 2005; Kallinikos, 2004). This goes for the material nature of the focal technology studied (and differences that results from these being, for example, energy technologies that span several domains of hardware and software vs being easily modifiable physical objects), as well as the production systems, tools, and infrastructures that designers and users use to shape them (cf. Cambrosio and Keating, 1995; Galison, 1997). This is to say, BOAP insists on carefully investigating the different materialities and their effects in different sites and times of a technology's life and carefully reflecting on what this entails for the overall research design-something more often claimed than carefully done in social studies of technology.

7. Through the above, BOAP studies seek balanced and empirically adequate accounts of what different actors do rather than assume, for instance, that key design decisions must have been made by designers (for, as we discuss below, they may be made by users).

8. The detailed dynamics of sociotechnical change are attended to, both empirically and theoretically. This has been the focal interest in all BOAP research to date. It has involved pursuing a detailed understanding of change in different settings and moments. This is at odds with resorting to high-level 
depictions of sociotechnical change. We discuss below the risk that widely adopted conceptions of studying sociotechnical change, such as social construction, mutual shaping, or systems transition, used as a template to characterize the relevant processes and net outcomes, may be used as an excuse for only using high-level generalization and occluding the detailed processes that factually constitute it, often against a declared intention to do so (for example, Bijker, 1995; Schot and Geels, 2007; Geels et al., 2016).

\subsection{Ecologies of actors and their interstices: theory bridging as the ninth BOAP characteristic}

The notion of ecologies of actors in the present use of BOAP is, in this book, rooted in a wider "ecological" view of sociotechnical relations, typically informed by Chicago sociology and the ensuing institutional analyses of professions and symbolic interactionist research on work and technology (e.g., Strauss, 1978, 1993; Star, 1995; Abbott, 2005; Clarke and Star, 2003; Akera, 2007). Abbott (2005: 248-249) provides a set of particularly clear articulations of the position of ecological sociology. In respect to broader social theory he remarks:

When we call a set of social relations an ecology, we mean that it is best understood in terms of interactions between multiple elements that are neither fully constrained nor fully independent. We thus contrast ecology with mechanism and organism on the one hand and with atomism and reductionism on the other. The latter contrast is straightforward and general: ecology involves some kind of relation between units whereas atomism and reductionism involve only qualities of units themselves or of their aggregates. With mechanism and organism, the contrast is more specific. When we encounter complete and routine integration in the social world, we employ the metaphor of mechanics, as in the "rule-governed systems" of role theory, for example. When we encounter systems whose elements move together in flexible homeostasis, we use the metaphor of organism, as in structural functionalism. By contrast with these two, in ecological thinking, the elements are not thought to move together at all; rather, they constrain or contest each other. "Ecology" thus names a social structure that is less unified than a machine or an organism, but that is considerably more unified than is a social world made up of the autonomous, atomic beings of classical liberalism or the probabilistically interacting rational actors of microeconomics.

In the current theoretical landscape, an ecological view of social phenomena finds close parallels in STS conceptualizations that acknowledge that complex, large-scale changes involve not only "hot" sociotechnical relations, in which the nature of actors is uncertain and their relations are fluctuating, and "cold" sociotechnical relations, where technology characteristics, markets, institutions 
and organizations have already congealed into stable and distinct entities (Callon, 1998), but also, and predominantly, varying topologies that feature mixtures of the two (Star, 1995, 1996; Jørgensen, 2012; Hyysalo et al., 2019a). In such a view, functionalist system conceptualizations should be heralded for their insistence on the remarkable power and obduracy of solidified sociotechnical structures, and the corresponding difficulties of system change (see, e.g., Hoogma et al., 2002; Geels, 2002). Again turning to Abbott: "[n]o social world ever exists without a pre-existing topology of some sort" (Abbott, 2005: 249). At the same time, the systems-oriented concepts are seen to remain deficient in understanding highly dynamic and emergent processes because they lack the means to address the various forms of agency and interplay between actors who negotiate the spaces and timings of the processes of far finer granularity (but no less complexity) that comprise whatever is realized of the change (Garud and Gehman, 2012; Spinardi and Slayton, 2015).

Conversely, theorizing that rests on actor networks as ever-expanding sets of overflows without consideration of pre-existing obdurate framings is seen to lapse toward sociotechnical voluntarism. Amid their socio-material assemblages, humans and other entities "make their own histories, but- to modify the Marxian dictum-in that making they produce larger structures that in turn render them unable to make those histories under conditions of their own choosing" (Abbott, 2005: 254).

Thus, in theories popular in SST and innovation studies this epistemological stance is different to both ANT (Latour, 1987, 2005) or, for instance, the MLP (Geels, 2002; Geels and Schot, 2007). ANT progresses from actants and the networks they form, leaving all issues of topology and structuration to be empirically settled, and it does not have pre-existing or generic conceptual registers to address topologies and structures (i.e., it relies on what has come to be called "flat" ontology). The MLP, in turn, presupposes a pre-defined structural ontology that study findings have to populate. In contrast, ecological views acknowledge the existence of previously identified social entities as sensitizing concepts that orient empirical inquiry towards topologies and ecologies, and suggest empirical points of entry but do not assume that these necessarily take a pre-defined structure or shape and thus leave the outcome analysis of both ecologies and topologies to be empirically built for the topic at hand, not structurally pre-determined (Strauss, 1993; Star and Clarke, 2003; Akera, 2007).

To understand this view better, we elaborate on some of the key concepts in more detail.

Ecologies of actors result in mutually defining lines of action by the actors involved in an event (Blumer, 1969; Strauss, 1993) and the patterning of events that have resulted in more durable social institutions and the topologies of power, resources, skills, constituencies, and commitments that have resulted from these (Blumer, 1969; Strauss, 1993; Becker, 1982; Clarke and Star, 2003). Ecologies of actors are typically populated by a range of differing sociotechnical entities, some of which are nested and others which are not, or which are only partially nested. Organizations, social movements, 
electoral constituencies, professional and industry associations, science labs, start-up companies, families, and governmental agencies all have distinct characteristics and therefore different capacities for action. Regarding an event or an arena, the people in these sociotechnical entities are typically aware of each other and the patterns of previous actions (Becker, 1982, 1998). They also tend to have complex interrelations that reach, in time and space, beyond a single event or arena (Star, 1989a). The above has a propensity to result in many-to-many translations that extend over time rather than being one-time contestations or translations of interests (Star, 1989b; Bowker and Star, 1999).

Arenas for social action refer to sociotechnically constituted sites rather than simple geographic locations, wherein the current and renewed order between actors is negotiated (Clarke and Star, 2003). Arenas should be seen as settings that feature some measure of stability and recognizability for the actors involved in them. Arenas can be at various stages of formation, ranging from the emergent and fluid networks that are well depicted by the "arenas of development" concept (Jørgensen, 2012) to established arenas that feature pre-existing sets of (bundled) issues and rules, and require certain skills, resources, and materials in order for there to be competent action.

Peripheral participation and multiple memberships are commonly recognized in the study of sociotechnical phenomena. Social life is seen to be made out of events, and stability ensues from the gradual formation of boundaries leading to entities, rather than entities already coming with boundaries (Abbot, 1995). This means that social entities — such as arenas, social worlds, or organizations - are seen to have "porous boundaries" and varying centrality of membership. Social formations may invest in guarding their membership, but people, objects, and infrastructures hold membership in multiple social worlds by default (Strauss, 1993; Clarke, 1998; Bowker and Star, 1999). Some such memberships result in go-betweens, others in boundary spanning, and yet others transform one or several social entities via mutual influences (Star, 1989b; Becker, 1982, 1998).

Given that ecologies of actors present very complex and rich research terrains, the focalization of research on particular sets of issues and actor groups is a practical necessity. For instance, Abbott's studies of the linked ecologies of professions are focalized on major and minor professions, the turfs they occupy in society, their emergence and waning, and the processes and results of negotiated order between them (Abbott, 1988, 2005). Clarke's study of reproductive medicine focuses on the interactive developments between key social worlds, the arenas between them, research programs, and the laboratories and instrumentations involved (Clarke, 1998, 2005). Star and Strauss (1999), in turn, focused on the invisible work inside research laboratories. The focalizations can be of different intensity; the study of invisible work is a more fine-grained focalization than that of Clarke, which in turn is more fine-grained than that the analysis of changing institutions by Abbott, yet they all expand out to the relevant ecologies of actors and processes therein in order to make sense of their focal interests. 
The focalization leads us to articulate a ninth BOAP guidepost that is particularly salient in the present line of investigation, namely:

9. Theory and the research tradition cross-linking. BOAP investigations typically mean deploying a number of mutually complementary studies on different aspects of the biography of technology, and over different time frames of analysis. Each focalization is typically relevant for an existing body of research and theory building. A common characteristic of a BOAP investigation is that its sub-study designs tend to be informed by the research templates and theories relevant to the sub-study, but these become linked and are critically compared within the scope of the overall BOAP investigation. The ensuing cross-linking and qualifying insights are pursued between different detailed foci (and literature), as well as between the detailed sub-studies and broader but more coarse grain-size analyses and theory framings (Pollock and Williams, 2008; Hyysalo, 2010), as well as varying fluidity of the social settings and structures studied. Such a strategy is neither naively inductive nor based on the testing of hypothesized theoretical models but can navigate between the two (Hyysalo et al., 2019).

To concretize: in the present book we began with a user-innovation focus and the research framing that this research community has established (Hyysalo et al., 2013a, 2017; Mattinen et al., 2014). We did not just reproduce it though; we purposefully gathered data on related actors, dynamics, and interstices in our ethnographies and interviews. This next led us to do a study on the embeddedness of user innovation in internet peer communities using an S\&TS framing for knowledge infrastructures (Hyysalo, 2013b); a comparison of ownership and technical configurations (Juntunen and Hyysalo, 2015); the adoption of renewables using a domestication framework (Heiskanen et al., 2014; Juntunen, 2014b); the internet peer communities influence to the overall diffusion and transition process (Hyysalo et al., 2018); historical analysis of heat pumps in Finland using a transition framing (Lauttamäki and Hyysalo, 2019); and finally, we connected the sub-studies in an overview study of users in a heat-pump transition (Chapter 5). All these sub-studies are cross-linked in the present overall volume of this BOAP investigation.

\subsection{Methodologically speaking: coverage of sociotechnical phenomena by analysis unit, observation unit, and data}

The closure effects and core markers discussed above have been subject to discussion and debate for two decades now. As such, it has become evident that there are now communities of researchers who have experienced first-hand the framing effects through studying the same technology in multiple settings and recognizing the importance of this issue. But it has become equally clear that others - perhaps those without a personal experiential basis - tend to go to considerable lengths to find alternative explanations for the study design's 
framing effects or to simply seek to avoid the issue altogether. This being the case, let us articulate the issue with a degree of formality through examining the relationship between analysis units and observation units in different strands of innovation studies and technology studies and the BOAP approach.

\subsubsection{Analysis units, observation units, and data in studies of technology adoption and innovation}

A good entry point for examining the analysis and observation units in innovation studies is to examine survey-based studies of innovation. These have readily graspable analysis units, observation units, and data, and in addition they continue to be widely deployed (in fact, well beyond the application area that their study set-up permits, as demonstrated below). Survey-based innovation studies can be exemplified by the OECD's community innovation survey and innovation diffusion survey. In both surveys types, the set-up is similar: the analysis unit is the adoption of innovation (the diffusion of innovation) or the introduction of innovation (a community innovation survey). The observation unit in both is the survey response to a set of questions based around "Have you adopted a particular innovation?" or "Have you introduced an innovation?" Insofar as the entity to whom this question has been directed is the person who answers the survey and the invention is discreet and clearly recognized, there is strong coverage between the phenomenon studied (the adoption/introduction of the invention by the person or company), the analysis unit by which it is methodologically operationalized (the adoption/introduction of novelties as reported by the respondent), and the observation unit, which the real-world counterpart to the analysis unit (a response to the survey question on whether or not one has adopted/introduced a novelty). The survey does not necessarily give very rich or detailed data on the observation unit but gives sufficient data for the purpose at hand. Figure 2.1a illustrates this with respect to a diffusion survey.

However, the coverage between the phenomenon, the analysis unit, and the observation unit changes if the knowledge interest concerns a more complex entity, such as a community or an organization. In Figure 2.1b, we see

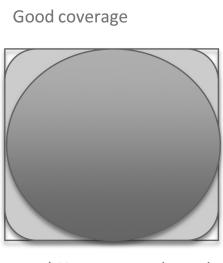

a) Has person adopted

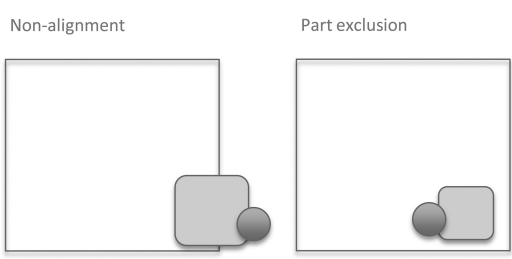

b) Has somebody adopted

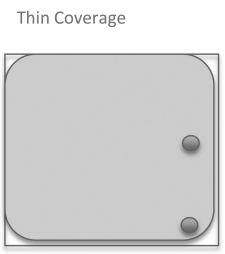

d)Organizational adoption

Figure 2.1 Coverage between the analysis unit, observation unit, and data in technology adoption research. 
how the situation changes if the respondent to the survey answers on behalf of others without first-hand certainty of what exactly has happened, the default case in any organization or community of more than 50 people. The individual respondent is assumed to somehow be able to speak for the whole organization, and the research takes the organization as the unit of analysis without considering differences of access to information and different orientations within the organization that affect its observation unit.

The situation is only slightly improved if, say, two respondents in an organization of 50 employees answer the survey regarding the typical 1-3-year radius used in these surveys. The analysis unit, the adopting/introducing organization, is now covered by an observation unit that is aligned, but the coverage is rather thin and easily amenable to the hollow-arch bias discussed above (Figure 2.1d). These are well-known weaknesses of diffusion studies regarding organizational diffusion (Rogers, 2010) and apply to survey-based innovation studies as well.

If the novel item is ambiguous or can be decomposed, added onto, or integrated in any way (Figure 2.1c) the analysis unit of "adoption" and its relation to the observation unit becomes undifferentiated-respondents can interpret adoption as only straight adoption or also as partial, piecemeal, and innovative adoption and answer accordingly (Kohtala et al. 2020). This is an equally wellknown problem in diffusion research (Rogers, 2010; Helminen et al., 2018), and, as we discuss in Chapter 3, it also features in user-innovation research done using surveys resulting in propensity to downplay the amount of local modifications and adaptations by users.

When thin coverage of organizational adoption by the observation unit is combined with an undifferentiated analysis unit in regard to adoption/adaptation, the reliability (as well as the validity) of the results is low regarding the phenomenon under study (Rogers, 2010). In research on the organizational adoption of complex technologies, such as software products, such a lack of fit has been remedied by in-depth interview and ethnographic studies. These allow for the use of a large series of observation units, covered by rich data, with the help of which issues such as adoption and adaptation (and over 20 other adoption moves) can be identified and reliably studied (DeSanctis and Poole, 1994; see Figure 2.2a).

While richer data and more numerous observation units add reliability, they also prompt researchers to ask new questions. The improved coverage allows moving from the question of whether or not adoption or adaptation has happened onto questions about how it happened and what happens in adoption. This shift took place in the 1990s (Silverstone, 1992; Berger et al., 2006; DeSanctis and Poole, 1994; McLaughlin et al., 1999) and found its parallel in studies of innovation, which moved beyond asking if and what, for instance, users or producers innovate, moving onto a processual understanding of how their innovation happens (van de Ven, 1999; Russel and Wiliams, 2002).

But changing the question resets the requirements for coverage. Answering these new questions adequately requires more fine-grained units of analysis and the corresponding observation units and data to cover them. The same 


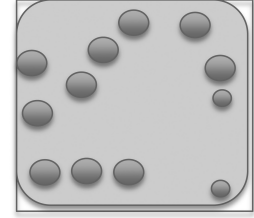

a) Adoption by orgnization

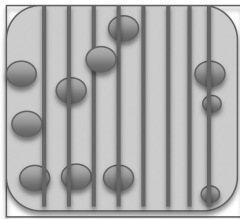

b) Adoption process

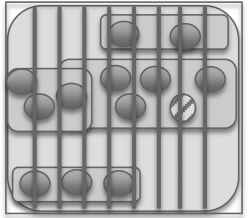

c) Adoption process and sub-processes

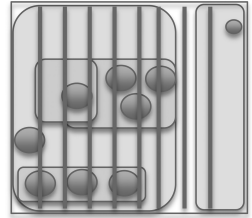

d) Implementation and impact studies of IT adoption

Figure 2.2 The analysis units and observation units in more in-depth studies of innovation and organization.

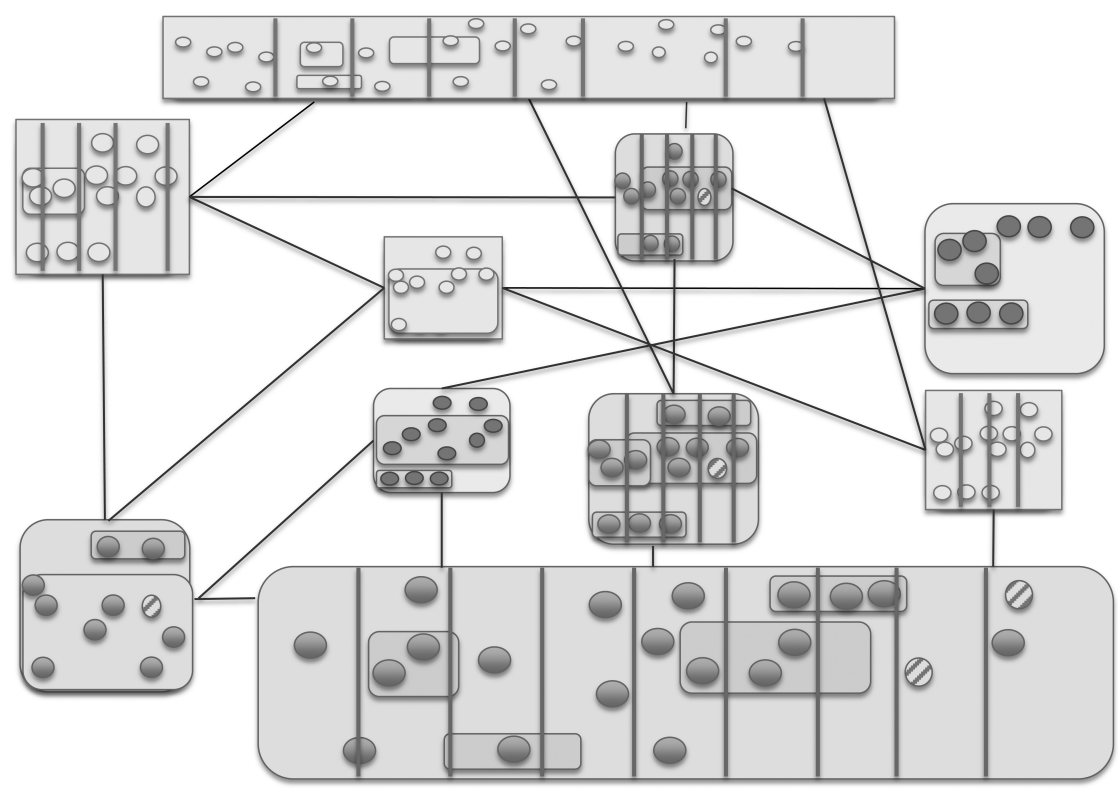

Figure 2.3 BOAP investigation designs several lines of sub-study of different aspects of sociotechnical change.

ethnographic adoption study that features an excess of observation units and data for answering whether or not a community or an organization has adopted/adapted a novelty system (Figure 2.2a) can be inadequate regarding the process of adoption if, for instance, observation units are missing data on some part of the process or if the data in many parts concerns, say, only one aspect of the process, such as how the technology is altered or how the users 
evaluate the technology (Figure 2.2b). Put methodologically, process questions require, by default, a series of observation units and typically also several analysis units on different kinds of events and phases in order to cover the relevant and typically interacting phenomena involved. (Figure 2.2c).

This then leads us to the rationale for BOAP studies: if one is to study change over time and beyond just a single organization, what kind of study set-up is required? The study design framing effects that BOAP is concerned with can remain systematically unrecognized. In diffusion studies, it took next to three decades to admit that diffusion surveys and the way in which diffusion research thought of the adoption process would not work for complex technology or for complex organizations (Attewell, 1992; McLaughlin et al., 1999; Rogers, 2010) and even fell short of understanding the cultural appropriation processes (Silverstone et al., 1992; Rogers, 2010).

When Pollock and Williams (2008) compared the findings from hundreds of packaged software implementation studies (typically conducted shortly after the implementation process with in-depth qualitative observation units and data sets) with hundreds of studies on software impact (conducted by asking the management survey questions later in the adoption process), the results were strikingly at odds: the latter produced an image of implementation as a productivity-enhancing improvement and the former portrayed it as productivitylowering turmoil in an organization. But when we compare the analysis units and observation units of these studies, we notice that the studies are not only asking different questions, using different theories, but were equally divorced with respect to when, how, and from what occupational groups their data of the "same" phenomenon is from (Figure 2.2d). To clarify the exact interrelation between the two types, comparative studies in the same project would need to reach the whole span of both studies, use both data gathering methods, and have the more detailed observation units' series for the whole process. This is not undoable but often rather tedious and thus not something that likely happens unless researchers are methodologically informed to do so.

Similar analysis unit, observation unit, and data issues apply to innovations. To cover more than the incidences of innovation by an organization, more numerous observation units would be needed, as was the case with organizational adoption. A major finding from the process studies of innovation has been that innovations are complex processes that tend to feature several follow-on innovations and reframings, span organizational boundaries, and have extended time frames-hence further complicating any attempt to inquire into their incidence using surveys and equally highlighting that the previous mainstream understanding of innovation, as borne out of studying how science was applied in corporate research environments, was largely an artifact of scant observation units and thin data that simply suppressed all the complexity and contingency of innovation from view (van de Ven, 1999; Russell and Williams, 2002; Poole and van de Ven, 2005; Garud and Gehman, 2012).

What BOAP brings into this research landscape is the realization that technologies evolve in organizations over several product generations, and so does 
their design, and so do the activities of various third-party actors in the market that shape both design and use. Previous innovation process studies had already shown that the phenomenon of innovation does not stop at the developer organization, but the BOAP findings underscore that it does not stop at the product launch or early-adopter sites either. The consequent further multiplication of the analysis units, observation units, and data presents a challenge to researchers. To study the process of innovation and sociotechnical change, we need to study the relevant set of locales and practices wherein the innovation is shaped (and conversely that are shaped by the innovation), hence the BOAP guideposts in the above section.

Given that innovation process studies, including BOAP studies, indicate time frames of years and decades for most innovation processes, scholars are faced with the sheer impossibility of, say, establishing a data track of videorecorded ethnography on all relevant observation units within all the needed analysis units in order to cover the span of times and sites within which an innovation is shaped. This entails a program to sample and compare insight from the different analysis units and observation units to understand innovation processes (Figure 2.3). As articulated in Sections 2.2 and 2.3, BOAP research designs rely on establishing arrays of analysis units and observation units with different levels of granularity. This zooming in and out is only possible when one has sufficiently rich and detailed data to do so. Figures 2.1 and 2.2 can be used to depict the situation. If one has Figure 2.2a- and 2.2b-type data for events A1 and A2, but Figure 2.1d-type data for event A3, one can use these to depict the events at the least-detailed level for A3-A1 and A2 are then just redacted to the same grain size to allow comparability, as if sieved through a "strainer" of the same coarseness (for a comparative biography study of data "sieving procedures," see Hyysalo et al., 2016c). However, if the data for A2 were in fact those of Figures $2.1 \mathrm{c}$ or $2.1 \mathrm{~b}$, its use in the same depiction becomes questionable. At best it can be treated as indirect evidence unless other data allows backing it up. The zooming out thus only becomes possible if one has enough factual reach and scope in the empirical data. In turn, zooming in is only possible when the density of observation units and data for these observations allows it.

\subsubsection{Theoretical case sampling and within-case sampling in the continuation of BOAP investigations}

The development of BOAP investigations may be more or less programmatic depending upon the availability of resources (e.g., staff time, the research funding environment) and access constraints. The beginnings of a BOAP investigation may not differ much from other innovation studies or STS research but, as the research progresses, previous research is extended to a string of further studies, building upon existing knowledge and the various ideas/issues that unfold from this work and reflecting upon puzzles and gaps in understanding 
and emerging theorizing. These continuation strategies can be discussed in terms of four types of theoretical sampling (Glaser \& Strauss, 1967).

The first type of theoretical sampling is the expansion of the units of analysis. This may arise because of multiple research concerns (such as the desire to cover internal variance within the field of study, addressing adjacent influences, or covering the more long-term dynamics such as the evolution of the studied technology and technology), or some other reason why a scholar would like to present a broader picture of one or several innovation processes. The ensuing questions typically concern which of the sites, times, and actors that influence the innovation currently remain as blanks or are suspiciously thinly covered. The theoretically sampling typically begins by re-examine the data one already has regarding the needed new observation/analysis units and, next, to assess if and what more data can be generated. This may mean another interview round, choosing some field-sites for further observation, data comparisons with peers, or devising a new research set-up for the upcoming years of research. A common strategy has also been to examine if prior studies could provide broader coverage from which one might zoom out. In the present book, this was done through following the Finnish heat-pump field for a decade while expanding the study focus in order to put the initial findings into a broader perspective.

The second theoretical sampling strategy is that of gradually shifting the focal perspective in follow-on studies so that they focus on new sets of relationships, locales, and types of actors identified as potentially relevant in previous studies. In the course of such a journey, both research questions and the relevant streams of literature are likely to change significantly. A good example is Pollock and William's $(2008,2016)$ focal shifts from studying the implementation of packaged software to their procurement, development, user groups, user communities, and industry analysts in order to gain a rounded understanding of the software's evolution. In the present study we moved from user-innovation in renewables to peer support, then to peer communities, onwards to usercreated information infrastructures and finally to user roles in affecting energy transition (Heiskanen et al., 2014; Hyysalo et al., 2013b, 2018).

The third theoretical sampling strategy concerns intensification through zooming in. Zooming in is typically motivated by a wish to understand a specific sub-phenomenon in a more nuanced way or to question an assumption made at a coarser level of depiction. Zooming in requires more dense data and is thus typically only possible to do in an uneven fashion. BOAP studies indicate that it is illusory to demand in-depth data on all sites and times of innovation but, equally, that one should not go about assuming that a given in-depth study would be the site where the interesting phenomenon happened unless one can show at a coarser level that this is probably the case. Hence the notion of "strategic ethnography" (Pollock and Williams, 2008) and a "focus on key junctions of change" (Hyysalo, 2004). In the present line of investigation several intensifications were pursued, for instance, intensifications to clarify the carbon footprint effects of user innovations (Mattinen et al., 2014; Heiskanen et al., 2015) and pathways to adopting S-RET equipment (Juntunen, 2014b). 
The fourth type of theoretical sampling is related to establishing focal interestcontext interrelations. Most BOAP studies focus primarily on a few interrelated aspects of innovation, such as in the present investigation role of users, the change in the artifacts, the shaping of the marketplace, and innovation communities. The rest of the innovation and underlying sociotechnical changes are typically examined more broadly as an enacted context for this focal interest and at a coarser level of depiction. The result is that research progresses through several parallel scales of inquiry ranging from the evolution of practices to episodic studies (of varying durations of minutes to months) (e.g., Hyysalo, 2010; Hyysalo and Usenyuk, 2015; Johnson, 2013).

Extending enquiry beyond single settings emphasizes the need for such extended theoretical sampling strategies in studying sociotechnical change. Different data types and sites of data collection have their own framing effects (Miettinen, 1993; Hyysalo, 2010; Murto et al., 2020a, 2020c). Ethnographic observation, recorded in field notes, and audio and video recordings, provides a first-hand experience of the realities of design and the use of technology. However, ethnographic understanding accumulates slowly and partially, and it is common that some processes may not-yet be readily recognized by the involved actors or research scholars and may only emerge over time or by contrasting different settings. Interviews provide a more focused method of eliciting knowledge but may be shaped by the interests and self-justification of the actors involved. The immediacy of the ethnographic insights that arise from field observation and interviews could bring to the surface particular conflicts, concerns, and events that appeared particularly interesting for research, and in this way they assist in analyzing other sources of data, such as documents (the effects of these BOAP theoretical sampiling strategies are schematically represented in Figure 2.3 study design - it is not a unified depiction but one that allows reasoning across different sites, aspects and granularities of analysis).

Similarly to other multi-site studies, BOAP research designs are built to allow for the further juxtaposition of different actors' narratives and perspectives and, in doing so, increase the trustworthiness and robustness of analysts' interpretations (Miettinen, 1993). The variety of the available data tends to grow with multi-sited and longitudinal investigations and allows both data and method triangulation (Denzin, 1989). The theoretical sampling strategies help to balance the framing effects in the accounts of the interaction created, and also, the extended scope of study tends to level out particular actor concerns, momentary hypes, or displays put on for the ethnographer when one enters the site over a sustained period.

Let us concretize this methodological treatise by briefly decomposing one well-known example and one recent case of a technology study with respect to the phenomenon, analysis units, observation units, and data in which we think BOAP sensitivity would lead to a significantly different interpretation of the study and the proposed concepts, and then we present and contrast this with the present study data sets and analyses. 
The study of agency in transition pathways by Geels et al. (2016) endorsed roughly the same ideas as those of BOAP: multiple layers of analysis, and zooming in and zooming out to study sociotechnical change. The study had twofold conceptual objectives. The first was to qualify Geels and Schot's (2007) concept of transition pathways by examining if the real-time transition pathways are as uniform as the long time frame historical case studies suggested and if they could, in fact, shift from one archetypical pathway to another. The second conceptual objective was to answer to the critique among technology studies scholars that the multi-level framework ignores the actor perspective and, in doing so, the enactment of sociotechnical change (e.g., Shove et al., 2012; Hyysalo, 2010; Spinardi \& Slayton, 2015). Empirically, the paper analyzes the UK's and Germany's renewable energy transition in 5-10 year phases, noting in each phase how the overall development had progressed and how each incumbent and renewable industry field and policy decision had changed it. The changes in the archetypical pathway types in the 20-year time span are then documented. The argument that the transition pathways, examined more in real time, are likely to be more complex is argued for convincingly. From a BOAP perspective, however, the ensuing first question becomes: would the earlier overall transition trajectories appear as uniform as originally depicted if historians had access to as detailed material available per five-year phase as that of the Geels et al. (2016) case? That is, are the neat overall trajectories in, for example, Geels and Schot (2007) mostly an artifact of scant data and thin analysis rather than that of rigorous theoretical abstraction? Regarding the second objective, Geels et al. (2016) noted that in their study they do not zoom in to study the enactment of change at policy setting or company level, and thus the "enactment" is discussed at the level of "nuclear operators," "the coal industry," "the wind energy sector," and "the government." In the BOAP view, this grain-sized data still remains at a scale where only the gross outcomes of enactments may be visible and where no such social actors exist to devise any actions and thus "enact" transition on the ground (cf. Garud \& Gehman, 2012). Not at all belittling the usefulness of the meso-perspective that Geels et al. (2016) pursue, an actor perspective would entail depicting how particular natural or corporate bodies — such as organizations, associations, collective actor groups, policymakers, and so on-would have perceived and sought to act in relation to the transition pathway (cf. Garud and Gehman, 2012; Höyssä and Hyysalo, 2009). The framing of the study in relation to its execution thus still appears, according to the BOAP perspective, as a lower-level systems view rather than that of an "actor perspective" on the transition, even if the analysts conducted more focused and real-time systems analysis. The positive message from BOAP would be that there may be a good possibility to next accumulate more detailed data sets in order to move into the study of the actual actor perspectives. With more fine-grained data, one could zoom in and out on the process of enactment and sociotechnical transition, and between the grain size of actor negotiations and contingencies, and the currently used countrylevel view of sociotechnical transition. The meso-level depiction Geels et al. 
2016 had achived would provide excellent contextualization for these studies. Hence, in the case of Geels et al. (2016), the empirical study may be well done, but its conceptual interpretation stretches beyond the evidence they have at hand as it tries to account for phenomena that are only ever likely to become visible once one further zooms-in to a still finer grain size.

The BOAP study in the present book presents such zooming to finer grainsizes and has been anchored to heat pumps in the residential housing sector in Finland. By anchoring I mean that all the sub-studies and analyses include an analysis of residential sector heat pumps, even though the innovation- and adoption-related sub-studies reach out to other S-RETs in Finland (pellet burners, solar collectors, and solar PV) and some of the study aspects make international comparisons in order to contextualize the innovation, diffusion, community, and transition phenomena investigated. The characterization of Finland with respect to S-RET developments and the specific data and analysis procedures are reported in the Appendix of this book.

In the next chapters we move onto examining the yield of the BOAP approach in order to understand the user activities in renewables innovation and transition, and return to reflect on this deployment of BOAP in the concluding chapter.

\section{Note}

1 The chapter introduction and Section 2.2 and 2.3 build on research that has previously appeared in Hyysalo, S., Pollock, N. and Williams, R., (2019a). "Method matters in the social study of technology: Investigating the biographies of artifacts and practices." Science and Technology Studies 32 (3) 2-25. 


\title{
3 Initial focus
}

\author{
User innovation in sustainable \\ energy technologies
}

\subsection{Introduction: the active and inventive users of renewable energy technologies ${ }^{1}$}

Energy innovation has traditionally appeared as a context dominated by research institutes and large incumbent companies, exemplifying a science push in technological change. Technological improvements are seen to emanate from research laboratories and find their way to end consumers via suppliers, retailers, assemblers, maintenance providers, and promotional agencies (Shove, 2010). In this scheme of things, consumer attitudes and perceptions of technology act as predictors of users' adoption (Klein and Rosenberg, 1996; Rogers, 2010; Coombs et al., 1987) and the key issue becomes how to overcome the "social" or "non-technical" obstacles to the flow of adequate energy technology and knowledge into practice (Shove, 2010).

But things are changing, and the reality may have never been so onedirectional. Research on residential energy use has consistently found great variations in energy use among similar households (Lutzenhiser, 1993; Guerin et al., 2009) resulting from what consumers do with their products and systems (thermostat settings, the number of showers and the length of showers, leaving lights on, etc.). And rather than following on simply from energy attitudes or diligence to adopting the technology, many differences result from how end-users adopt and adapt the technologies to their local conditions and the particularities of their houses and everyday practices (Shove et al., 2007; Caird and Roy, 2008). The importance of skillful adoption and finding a well-suited blend of appropriate technology options and energy practices has thus been raised to the fore (Heiskanen et al., 2010; Raven et al., 2008).

Similarly important are activities and skills in DIY house and energy efficiency renovations including smaller and larger adaptations to the technology in order to make them suited for the local conditions (Caird and Roy, 2008; Hargreaves et al., 2013; Shove et al., 2007; deVries et al., 2016). This is particularly the case with S-RETs that are directly associated with the existing building stock. The standard technology needs to be fitted to different, country-specific variations of housing, climate, and regulation, as well as to the often considerable variation that results from the particularities of

DOI: $10.4324 / 9781003133919$ 
residential buildings and house owners' everyday practices (Heiskanen et al., 2014; Nyborg, 2015; Judson et al., 2015). Capitalizing on such innovationsin-practice has been seen as part of "Grassroots innovation" (Seyfang, 2010; Heiskanen and Lovio, 2010).

In contrast to the centralized forms of energy provision that leaves only relatively passive roles available to consumers, S-RET systems give their users firsthand access to observe, modify, and improve their equipment. This can result in most thoroughgoing consumer engagement with their energy technology, namely the development of new-to-the-world innovations to the renewable home heating equipment itself. Such extensive citizen innovation activities have been documented in the early formative stages of renewable energy technology development. The role of grassroots communities in the wind turbine development in Denmark (Ornetzeder and Rohracher, 2013; Nielsen, 2016), the emergence and maturation of solar collector development in Austria, and user roles in passive house development in Germany are well documented (Ornetzeder and Rohracher, 2006). In the course of this chapter we see how such consumer innovation is not limited to early technology development stages and exceptional localities, but can be found in 'follower' countries as well and also in the later stages of technology development.

\subsection{Consumer innovation and its diffusion}

In a broader view, the inventive energy consumers are not exceptional. Users in many other walks of life have been documented to develop technology on their own, both with regard to industrial products and consumer products (for overviews, see von Hippel, 2016; Bogers et al., 2010; Gambardella et al., 2017). In specialist communities, as many as $19-36 \%$ of the users of industrial products and $10-38 \%$ of the users of consumer products have been found to modify products (for a review, see von Hippel, 2005). In representative surveys of the populations of, for example, Japan, the US, the UK, Canada, and Finland, 3.7-6.1\% of consumers reported having created or modified some of the equipment they use (von Hippel, 2016). The sum totals of their reported expenditure (in the US: $\$ 20 \mathrm{bn}$ ) range from 13\% of the total R\&D expenditure on consumer products in Japan to $144 \%$ of the total R\&D expenditure on consumer products in the UK. These users often freely or selectively reveal their innovations within their communities, as well as to companies, creating the phenomenon of open user innovation (Jeppesen and Frederiksen, 2006; Piller and West, 2014). Here user denotes any user who directly benefits from an innovation, be this in a professional or consumer context, whereas consumers are a non-professional and non-organizational subset of users.

Because of the large proportion of users who develop or modify products and the great amount of time and money they put into their projects, the diffusion of consumer innovations is one of the recent areas of interest in user and open user-innovation research (De Jong et al., 2015; Piller and West, 2014; von Hippel et al., 2017). There are indications that the spread of user innovations 
could be structurally hampered. Unlike producers, users do not have to invest in selling the innovation to others to benefit from it - they are innovating for themselves (von Hippel, 1976). From this it follows that efforts to diffuse the innovation may come as something extra and require that users appropriate a new role-either helping others (Freeman, 2007; Habicht et al., 2013), raising their professional profile (von Hippel et al., 2017), or turning into entrepreneurs (Shah and Tripsas, 2007). The last option aside, the gains users may enjoy from their effort to render their innovation diffusible may not be appealing, even if their innovation was socially valuable. "Under-diffusion" may thus prevail, which may present a market failure from a welfare economics point of view (De Jong et al., 2015; von Hippel et al., 2017). For example, Von Hippel and DeMonaco (2017) found that user innovators in medical drugs and devices seldom invest time in publishing their inventions in medical journals but rather only spread them through word of mouth. Similarly, in extreme sports many consumer innovations are initially rough design-wise, and wider adoption only occurs after additional efforts to make them more usable (Baldwin et al., 2006; Hyysalo, 2009). These conditions assumedly equally affect the consumers who innovate in the S-RETs. The research on the diffusion of consumer innovations in S-RETs and energy efficiency remains nascent, basically being limited to documenting that some diffusion has happened (Ornetzeder and Rohracher, 2006, 2013; Nygren et al., 2015; Gavin and Sunikka-Blank, 2014).

At the same time, the increasing digital connectivity among peers provides a range of communication channels to connect physically separated users. Various forms of user communities - ranging from online and consumerdriven hobbyists (e.g., Hyysalo et al., 2013a; Jeppesen and Frederiksen, 2006; Jeppesen and Molin, 2003; Marchi et al., 2011; Haavisto, 2014) to off-line and professional communities (Desouza et al., 2007; Usenyuk et al., 2016)-are supportive of sharing with peers, creating innovations together, and adoption from peers (Jeppesen and Fredriksen, 2006; Hienert et al., 2014; Hyysalo and Usenyuk, 2015).

The nature of the adoption of innovation by peers can have important variations, leading us back to observations of what consumers do when they adopt a new technology. As noted in Chapter 2, since the 2000s diffusion research has increasingly moved beyond surveys of the adoption decision onto detailed studies of actual adoption processes (Rogers, 2010), as adoption was commonly found to include adaptations, including resignifying, repurposing, adding on, modifying, or substantially redesigning the technology (Agarwal, 1983; DeSanctis and Poole, 1994; Douthwaite et al., 2001; Fleck, 1993b; Juntunen, 2014a; McLaughlin, 1999; Kohtala et al., 2020). Adaptation is asserted to increase the adoption of innovation by making it possible to adjust it to the cognitive, social, and material needs of the adopter (Agarwal, 1983; Fleck, 1993b; Rogers, 2010) and has been found to present an alternative to "straight transfer" diffusion in cases when users can turn to adaptation (Agarwal, 1983). An even more thorough blending of innovation and diffusion- "innofusion"-has been documented in rural wells, health programs, industrial robotics, multimedia 
and digital environs, agriculture, and vehicles (De Laet and Mol, 2000; Fleck, 1993b; Hyysalo and Usenyuk, 2015; Williams et al., 2005). It follows that attention should be given to the form of the innovations and the form in which they are communicated to peers, as well as to the form in which these innovations have then been adopted/adapted by peers.

The research interests in this chapter are thus twofold. First, we are interested in whether consumers innovate in an S-RET after the initial development stages, and if so where in the technical system can they innovate and how do they gain the competencies needed to do so. Second, we are interested in how the innovations by consumers diffuse, do they do so via commercial routes or among peers, and what forms does peer diffusion take and why?

These questions mean clarifying if the innovations by consumers make a difference by adding to the variety of technical solutions available in a given S-RET's development and diffusion. We shall also lay the ground for Chapters 4 and 5 by beginning to address the issue of what else is being created by consumers' innovation projects beyond just the solutions, as competence building and peer support may play important roles beyond their roles in innovating.

The chapter is structured as follows. We first examine what users are inventing in sustainable energy technologies and examine where in the technical systems users are able to invent and what issues appear to channel or curb users' inventive actions (Section 3.3). We then focus on the potential of user inventions in expert evaluation and in the LCA analysis of selected innovations, followed by the analysis of their diffusion (Section 3.4) and the chapter's discussion (Section 3.5).

\subsection{Consumer inventions in Finnish S-RET systems}

The activity of citizen end-users in energy-related adaptations is commonly thought of as being limited to add-ons and DIY renovation, using power tools and the help of DIY videos found on the internet (Shove et al., 2007). This would boil down to technically simple additions, such as placing a sledge under an outdoor air-source heat pump (ASHP) unit in order to remove ice in the winter or building housing for an ASHP in order to make it more aesthetically appealing (Figure 3.1). Such simple add-ons should not be belittled as they ease the use and uptake of new technology and its fit with preferences of its adopters.

However, the user inventions in S-RET technologies also include commercialized products and the very technically sophisticated rebuilding of machinery (see Figure 3.1, bottom row); in fact, they span a whole range of additions, adaptations, reinventions, and new designs, which we discuss in detail below.

\subsubsection{User inventions in air-source heat pumps}

In our search we found 79 consumer innovations in ASHPs. Thirty of these were system-level designs that spanned several subsystems of the technology. 

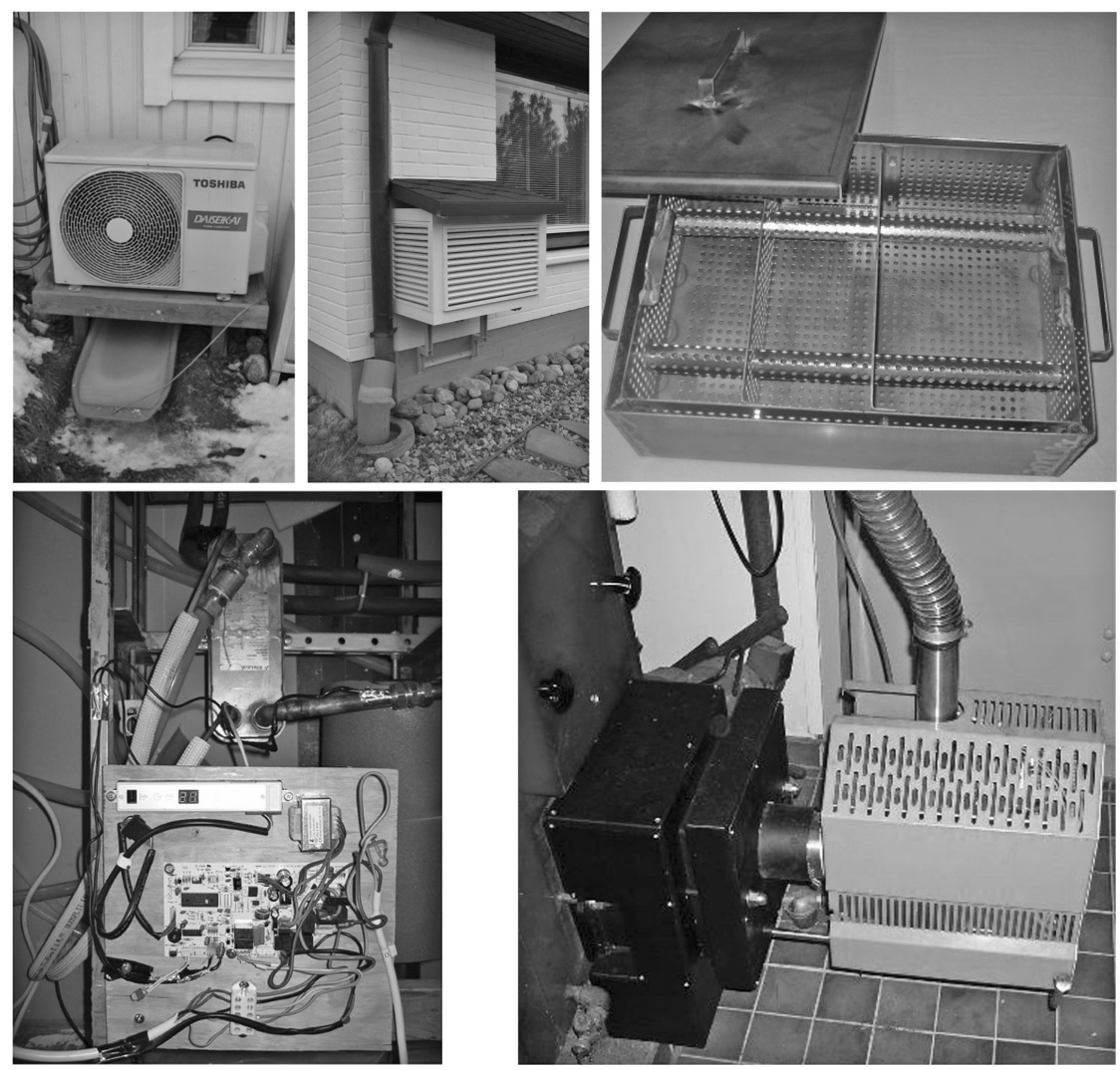

Figure 3.1 Examples of user designs in S-RET systems. A sledge used for ice removal (top left ${ }^{2}$ ), a housing for an air-heat pump (top middle ${ }^{3}$ ), and a wood pellet burning basket (top right ${ }^{4}$ ). An example of an advanced heat pump (bottom left ${ }^{5}$ ) and a wood pellet burning user project (bottom right ${ }^{6}$ ). Images freely available on the internet, research use granted by Finnish law.

We identified 25 user modifications, technical redesigns that were limited to one subsystem. Just as important were 24 cases where user inventiveness was best described as user add-ons, repurposing, hacks, relocating, or workaround. Let us first give short examples in order to characterize user inventions in these categories and then present a more encompassing view of these inventions in Table 3.1 that classifies the inventions according to subsystems of the ASHP technology.

An example of user design: turning an ASHP into an air-to-water heat pump (ASWHP). Several people turned ASHP models into ASWHPs by removing the ASHP's indoor convector unit and connecting the outdoor air-source collector into a liquid circuit in the water central heating of the house. The design required reconfiguring the coolant circuit, building the indoor circuit heat exchanger, adding new sensors and a control logic for both indoor and outdoor units, creating reference data for a successful control, etc. While the 
Table 3.1 User inventions in ASHPs

\begin{tabular}{|c|c|c|c|}
\hline $\begin{array}{l}\text { The part of the } \\
\text { technical system }\end{array}$ & The subsystem & $\begin{array}{l}\text { The no. } \\
\text { inventions }\end{array}$ & $\begin{array}{l}\text { An example of the invention by } \\
\text { consumer }\end{array}$ \\
\hline $\begin{array}{l}\text { An outdoor ASHP } \\
\text { unit }\end{array}$ & Installation location & 2 & $\begin{array}{l}\text { An outdoor heat-pump unit is } \\
\text { installed in an empty space } \\
\text { below the roof or floor in } \\
\text { order to gain warmer source } \\
\text { air }\end{array}$ \\
\hline An ASHP unit & $\begin{array}{l}\text { Installation } \\
\text { conditions }\end{array}$ & 1 & $\begin{array}{l}\text { Covering the unit with a roof or } \\
\text { within an overall cover }\end{array}$ \\
\hline $\begin{array}{l}\text { An outdoor ASHP } \\
\text { unit }\end{array}$ & $\begin{array}{l}\text { A melting } \\
\text { mechanism for the } \\
\text { bottom cover }\end{array}$ & 9 & $\begin{array}{l}\text { Adding a resistor parallel to the } \\
\text { sensor }\end{array}$ \\
\hline $\begin{array}{l}\text { An outdoor ASHP } \\
\text { unit }\end{array}$ & $\begin{array}{l}\text { A melting } \\
\text { mechanism for the } \\
\text { outdoor collector } \\
\text { coil }\end{array}$ & 4 & $\begin{array}{l}\text { Using an IR heater to prevent the } \\
\text { formulation of frost and ice on } \\
\text { the coil }\end{array}$ \\
\hline $\begin{array}{l}\text { An outdoor ASHP } \\
\text { unit }\end{array}$ & $\begin{array}{l}\text { An add-on for the } \\
\text { outdoor unit }\end{array}$ & 4 & $\begin{array}{l}\text { Air input comes from a system } \\
\text { that is built from plexiglass }\end{array}$ \\
\hline $\begin{array}{l}\text { An outdoor ASHP } \\
\text { unit }\end{array}$ & $\begin{array}{c}\text { Channeling the water } \\
\text { out from the unit }\end{array}$ & 6 & $\begin{array}{l}\text { The construction of inclines using } \\
\text { hard-to-freeze material inside } \\
\text { the outdoor unit in order to } \\
\text { prevent ice from blocking } \\
\text { drainage }\end{array}$ \\
\hline $\begin{array}{l}\text { An outdoor ASHP } \\
\text { unit }\end{array}$ & $\begin{array}{l}\text { Handling water } \\
\text { outside of the unit }\end{array}$ & 4 & $\begin{array}{l}\text { Placing a plastic sledge below the } \\
\text { unit to store accumulated ice }\end{array}$ \\
\hline $\begin{array}{l}\text { An ASHP } \\
\text { refrigerant } \\
\text { system }\end{array}$ & Refrigerant fluid & 1 & $\begin{array}{l}\text { Adding propane to } 410 \mathrm{a} \\
\text { refrigerant to improve its } \\
\text { functioning }\end{array}$ \\
\hline $\begin{array}{l}\text { An ASHP heat } \\
\text { exchanger }\end{array}$ & A hot water tank & 4 & $\begin{array}{l}\text { A radiator hot water tank } \\
\text { constructed from an old oil } \\
\text { tank }\end{array}$ \\
\hline An ASHP system & Water circulation & 1 & $\begin{array}{l}\text { Finding a way to install pipes } \\
\text { closer to the floor surface } \\
\text { than in commercially available } \\
\text { models }\end{array}$ \\
\hline $\begin{array}{l}\text { An internal ASHP } \\
\text { unit }\end{array}$ & $\begin{array}{l}\text { Repurposing an } \\
\text { ASHP as an } \\
\text { ASWHP }\end{array}$ & 15 & $\begin{array}{l}\text { The removal of the internal unit, } \\
\text { replacing it with a water heat } \\
\text { exchanger; changes to the } \\
\text { control logic are required }\end{array}$ \\
\hline $\begin{array}{l}\text { The repurposing } \\
\text { of an ASHP }\end{array}$ & $\begin{array}{l}\text { Repurposing an } \\
\text { ASHP as a } \\
\text { geothermal heat } \\
\text { pump }\end{array}$ & 1 & $\begin{array}{l}\text { The outdoor coil is replaced with } \\
\text { a heat-exchanger panel }\end{array}$ \\
\hline $\begin{array}{l}\text { An external ASHP } \\
\text { unit }\end{array}$ & $\begin{array}{l}\text { Repurposing a } \\
\text { swimming pool } \\
\text { heat pump }\end{array}$ & 1 & $\begin{array}{l}\text { The utilization of recycled } \\
\text { material to achieve new } \\
\text { functioning }\end{array}$ \\
\hline
\end{tabular}


Table 3.1 Continued

\begin{tabular}{|c|c|c|c|}
\hline $\begin{array}{l}\text { The part of the } \\
\text { technical system }\end{array}$ & The subsystem & $\begin{array}{l}\text { The no. } \\
\text { inventions }\end{array}$ & $\begin{array}{l}\text { An example of the invention by } \\
\text { consumer }\end{array}$ \\
\hline $\begin{array}{l}\text { An internal ASHP } \\
\text { unit }\end{array}$ & $\begin{array}{l}\text { Repurposing the } \\
\text { internal unit }\end{array}$ & 1 & $\begin{array}{l}\text { Using the unit as a blow } \\
\text { convector }\end{array}$ \\
\hline $\begin{array}{l}\text { An outdoor } \\
\text { ASWHP unit }\end{array}$ & $\begin{array}{l}\text { Utilizing cooled air } \\
\text { from the outdoor } \\
\text { unit }\end{array}$ & 1 & $\begin{array}{l}\text { Summertime cooled air can } \\
\text { be tunneled back to the } \\
\text { apartment for cooling purposes } \\
\text { when the pump is just used for } \\
\text { heating hot water }\end{array}$ \\
\hline $\begin{array}{l}\text { An ASHP remote } \\
\text { control }\end{array}$ & $\begin{array}{l}\text { An add-on for an } \\
\text { internal unit }\end{array}$ & 5 & $\begin{array}{l}\text { A PC soundcard-based control to } \\
\text { replace the remote control and } \\
\text { increase the control distance }\end{array}$ \\
\hline $\begin{array}{l}\text { An internal ASHP } \\
\text { unit }\end{array}$ & Mechanical structure & 1 & $\begin{array}{l}\text { Spraying "noise killer" to reduce } \\
\text { the noise of the internal unit }\end{array}$ \\
\hline $\begin{array}{l}\text { An internal ASHP } \\
\text { unit }\end{array}$ & $\begin{array}{l}\text { Sensors in the } \\
\text { internal unit }\end{array}$ & 4 & $\begin{array}{l}\text { Adding transistors and a resistor } \\
\text { in order to hack the internal } \\
\text { sensor and achieve a lower } \\
\text { minimum temperature }\end{array}$ \\
\hline An ASHP system & $\begin{array}{l}\text { An add-on for } \\
\text { monitoring and } \\
\text { controlling the } \\
\text { overall unit } \\
\text { performance }\end{array}$ & 10 & $\begin{array}{l}\text { The utilization of weather } \\
\text { forecasts from the } \\
\text { Wunderground weather } \\
\text { service in order to optimize } \\
\text { performance }\end{array}$ \\
\hline \multirow[t]{2}{*}{ An ASHP system } & System-level design & 4 & $\begin{array}{l}\text { A DIY unit for industrial } \\
\text { buildings }\end{array}$ \\
\hline & Total & 79 & \\
\hline
\end{tabular}

energy saving is not usually on a par with a commercial ASWHP, the cost is only $10 \%$ of an off-the-shelf ASWHP.

An example of user modification and repurposing: adding a resistor to an ASHP to make it maintain an $8^{\circ} \mathrm{C}$ temperature. Several users fooled the indoor temperature sensors of their ASHPs (in various technical ways) in order to achieve a sought-after lower indoor temperature. In cold countries, garages, summer cabins, and other non-lived-in spaces are heated to non-freezing temperatures in the winter. Heat pumps would cut this maintenance energy use significantly, but only if rigged to work below their minimum specification temperature, usually $16^{\circ} \mathrm{C}$. The latest commercially available ASHPs support this type of functionality but at a considerably higher price than rigged cheap models.

An example of a user add-on to an ASHP: A remote control is provided with several ASHP models to control, for example, the temperature and strength of the air flow. When the home includes several energy technologies, home automation systems are often used to simplify the daily use of the systems. The 
home automation can be PC based and can monitor and control several systems within the house. Users developed an add-on USB-connected infrared box, which is used to replace the infrared remote control of the internal unit and control the heat pump via the automation system.

Let us now shift the perspective to consider the clustering of such inventions within the technological configuration of this technology. Table 3.1 discusses the extent and distribution of the found user inventions within ASHP parts and technical subsystems.

Most modifications have been dedicated to the outdoors unit's problems with freezing and to the replacement of the indoor convector unit with water circulation. What is salient, however, is that users were able to invent in virtually all parts of the technical system, including the coolant gas mixes, control logics, and system-level design. Only three parts of the configuration were left untouched by the studied inventing users: the insides of the compressor unit, the reversing valve, and the expansion device.

These user activities with ASHPs make it clear that some users' capacity to improve their energy technology is considerable. Alongside a few newto-the-world inventions with commercial potential, a fair amount of other limited improvements emerged. This is noteworthy as heat pumps are not the easiest or most likely sites for user invention because of their technical complexity, mass production, low configurability, manufacturer disinterest in niche development, equipment integrating several technology areas (coolant systems, electronics, mechanics, software, and, in ASWHPs, also plumbing), and the loss of warranty and insurance coverage upon making modifications.

In user forum discussions and in our interviews with the inventors, the inventions were seen to be spurred on by the inadequacies of most commercial models in regard to dealing with cold climates and the ensuing room for improving their energy efficiency and usability. The relatively cheap price of ASHP models, particularly of the lower-end models encouraged experimentation, as did advice received in user-run internet forums where heat-pump owners exchange experiences, ideas, and help.

\subsubsection{User inventions in ground-source heat pumps}

In ground-source heat pumps (GSHPs) we found four system-level designs, eight user modifications, and 14 user add-ons, repurposings, hacks, relocations, or workarounds. Let us again first present short examples to characterize user inventions in these categories and then clarify their range and extent (see Table 3.2) according to the subsystems of GSHP technology.

An example of user design: an ASHP with a ground-source outdoor circuit. The typical GSHP is connected to a water circulation based central heating system, which houses heated with direct electricity do not have. An air-heat-pump convector unit with a ground-source outdoor circuit enables a cost-competitive GSHP solution that suits this building stock. The outdoors unit of heat pump, as well as 
Table 3.2 User inventions in GSHPs

\begin{tabular}{|c|c|c|c|}
\hline $\begin{array}{l}\text { The part of the } \\
\text { technical system }\end{array}$ & The subsystem & $\begin{array}{l}\text { The no. of user } \\
\text { inventions }\end{array}$ & $\begin{array}{l}\text { An example descriptor of the user } \\
\text { invention }\end{array}$ \\
\hline GSHP & $\begin{array}{l}\text { An add-on for } \\
\text { monitoring and } \\
\text { controlling the } \\
\text { overall unit } \\
\text { performance }\end{array}$ & 8 & $\begin{array}{l}\text { Using a Linux USB drive for } \\
\text { storing automation data; } \\
\text { low power consumption } \\
\text { compared to a constantly } \\
\text { powered PC }\end{array}$ \\
\hline GSHP & $\begin{array}{l}\text { An add-on for } \\
\text { monitoring the } \\
\text { ground heat } \\
\text { exchanger }\end{array}$ & 4 & $\begin{array}{l}\text { Using extra temperature sensors } \\
\text { for measurements with a } \\
\text { ground heat exchanger }\end{array}$ \\
\hline $\begin{array}{l}\text { A GSHP outdoor } \\
\text { coil }\end{array}$ & $\begin{array}{l}\text { Coil location or } \\
\text { modified use of } \\
\text { coil and well }\end{array}$ & 5 & $\begin{array}{l}\text { The coil is embedded in } \\
\text { wastewater infiltration } \\
\text { ground }\end{array}$ \\
\hline $\begin{array}{l}\text { GSHP/geothermal } \\
\text { heat-to-air } \\
\text { pump }\end{array}$ & $\begin{array}{l}\text { System-level } \\
\text { design }\end{array}$ & 1 & $\begin{array}{l}\text { An air-heat pump with an } \\
\text { underground air/liquid pipe }\end{array}$ \\
\hline $\begin{array}{l}\text { GSHP } \\
\text { superheating } \\
\text { buffer tank }\end{array}$ & Water piping & 2 & $\begin{array}{l}\text { Changing the valve to a } \\
\text { thermostat valve to gain } \\
\text { improved performance }\end{array}$ \\
\hline $\begin{array}{l}\text { An outdoor GSHP } \\
\text { unit }\end{array}$ & Well & 2 & $\begin{array}{l}\text { Use of a plastic drainpipe to } \\
\text { build a maintenance well }\end{array}$ \\
\hline A GSHP add-on & $\begin{array}{l}\text { An add-on for the } \\
\text { control logic }\end{array}$ & 1 & $\begin{array}{l}\text { The transfer of exhaust heat to } \\
\text { the GSHP source field }\end{array}$ \\
\hline A GSHP add-on & $\begin{array}{l}\text { An external hot } \\
\text { water tank as } \\
\text { an add-on }\end{array}$ & 2 & $\begin{array}{l}\text { The system is designed to work } \\
\text { with a small domestic hot } \\
\text { water tank; a separate lower- } \\
\text { temperature tank is installed } \\
\text { for circulating water }\end{array}$ \\
\hline \multirow[t]{2}{*}{ Radiators } & $\begin{array}{l}\text { The heat } \\
\text { distribution } \\
\text { system }\end{array}$ & 1 & $\begin{array}{l}\text { Increasing heat distribution } \\
\text { with a fan with radiators }\end{array}$ \\
\hline & Total & 26 & \\
\hline
\end{tabular}

the control logic and connections between inside and outside units, are modified. The "GSAHP" user innovation is commercialized by Jääsähkö Oy in Finland.

An example of user modification to a GSHP. Some GSHP models utilize socalled superheater solutions in which the domestic hot water is heated to a higher temperature with one additional heat exchanger. The user changed the piping in the unit in order to circumvent the lower-temperature water tank through which the unit circulated the water. He channeled the piping outside of the unit and installed a temperature changer to the piping in order to keep the water temperature as constant as possible, regardless of the status of the heat pump. 
An example of a user add-on to a GSHP. One practice innovation to improve GSHP efficiency is to insulate its outdoor coil with snow. The outdoor coil of the GSHP is typically installed either vertically in a deep rock well or horizontally in the yard or in a field. In the latter installation method, the coil is 1-meter deep in the ground. In the Nordic environment snow can be used as insulation during the wintertime. When snow is accumulated in a heap above the installed field it prevents the ground from cooling from the outdoor air, and the coil stays in a warmer environment.

The overall amount of GSHP inventions has been smaller than in ASHPs, but on a slightly higher level when compared to the number of units installed in Finland (by 2016 ASHP 0.02/ GSHP 0.04\%). As with ASHPs, users have modified most parts of the GSHP systems, but the core unit, including a compressor, evaporator, condenser, and coolant liquids, has only been subject to alteration in two more encompassing designs. Based on the list discussions and interviews with inventors, the main reason lies in the substantially higher price of GSHP installation (up to $€ 30,000$ ), which inhibits citizens' behavior in regard to violating the integrity and warranty of the equipment. GSHP equipment is also more targeted to cold climates and thus features less obvious space for improvement. Nonetheless, as with the ASHPs local conditions feature variation that generic designs do not fully cater for and thus leave unexplored design space, on which some users focus. As with the ASHPs and wood pellet burner technology that we discuss below, most user inventors were active in the user-run internet forum dedicated to this technology. Similarly, cost reduction or improvements in performance were the expressed motives, and the common pattern is that most modifications concern fitting the technology to local particularities, be those particular opportunities, such as the possibility to easily use snow to insulate the horizontal collector field, or constraints, such as the lack of water central heating.

\subsubsection{User inventions in pellet wood burning systems}

In wood pellet systems we found 12 system-level designs, 51 user modifications, and 24 user add-ons, repurposings, hacks, relocations, or workarounds. Let us again first present short examples in order to characterize the user inventions in these categories and then clarify their range and extent (see Table 3.3) according to the subsystems of the wood pellet burning technology.

An example of user design: Building a wood pellet burner and control logics from scratch. Several users built either their own burners or control logics. For instance, a father first used his welding skills to build the hardware, and his son built the needed control logics with his software programming and electronics skills. The resulting design lights up faster than commercial alternatives and minimizes burning gases. Several other users already use this new design.

An example of user modification and repurposing: turning an old oil burner into a pellet burner. Several users modified and repurposed their old oil burners to suit pellet burning. These modifications differed depending on the oil burner 
Table 3.3 User inventions in wood pellet systems

\begin{tabular}{|c|c|c|c|}
\hline $\begin{array}{l}\text { The part of the } \\
\text { technical system }\end{array}$ & The subsystem & $\begin{array}{l}\text { The no. of user } \\
\text { inventions }\end{array}$ & $\begin{array}{l}\text { An example descriptor of the user } \\
\text { invention }\end{array}$ \\
\hline Pellet boiler & Burner & 11 & $\begin{array}{l}\text { Building the user's burner design } \\
\text { from metal }\end{array}$ \\
\hline $\begin{array}{l}\text { A pellet boiler } \\
\text { system }\end{array}$ & A pellet boiler system & 11 & Welding a whole new boiler unit \\
\hline A pellet boiler & Burner control & 2 & $\begin{array}{l}\text { Programming the software logics } \\
\text { and wiring the electronics for } \\
\text { the user's burner }\end{array}$ \\
\hline Pellet transfer & A pellet auger & 3 & $\begin{array}{l}\text { A pellet auger formed from a } \\
\text { single piece of rust-resistant } \\
\text { metal }\end{array}$ \\
\hline Pellet transfer & Cyclon & 5 & $\begin{array}{l}\text { A 100-liter barrel, attached to } \\
\text { a recycled, efficient vacuum } \\
\text { cleaner with a rubber chain }\end{array}$ \\
\hline Pellet storage & Indoor silo & 5 & $\begin{array}{l}\text { Using a big, used metal container } \\
\text { for building a daily pellet } \\
\text { storage }\end{array}$ \\
\hline Pellet storage & Outdoor silo & 9 & $\begin{array}{l}\text { Building a separate outhouse for } \\
\text { pellet storage }\end{array}$ \\
\hline A pellet system & Measurement & 5 & $\begin{array}{l}\text { Monitoring burner temperature } \\
\text { and a failure alarm }\end{array}$ \\
\hline A pellet system & Automation & 9 & $\begin{array}{l}\text { A graphical temperature } \\
\text { monitoring system with } \\
\text { 1-wire sensors }\end{array}$ \\
\hline A pellet boiler & $\begin{array}{l}\text { An exhaust gas } \\
\text { vacuum }\end{array}$ & 1 & $\begin{array}{l}\text { An old oil tank turned into an } \\
\text { exhaust gas vacuum }\end{array}$ \\
\hline A heat system & Hot water tank & 1 & $\begin{array}{l}\text { Building a } 40001 \text { hot water tank } \\
\text { out of rust-resistant metal }\end{array}$ \\
\hline Other systems & $\begin{array}{l}\text { Other pellet burning } \\
\text { systems }\end{array}$ & 23 & $\begin{array}{l}\text { Building a burner basket that } \\
\text { enables the flow of air in the } \\
\text { burning process }\end{array}$ \\
\hline Other & Pellet production & 1 & $\begin{array}{l}\text { Building a transferrable pellet mill } \\
\text { on top of a pickup truck }\end{array}$ \\
\hline Total & & 87 & \\
\hline
\end{tabular}

brand. The problem with most oil burners with respect to burning wood pellets is that the fire hearth and the ash compartment are too small. Modification and repurposing required extending the burner cavity of the oil boiler lengthwise to enable a larger flame and hence cleaner burning, a prerequisite of efficient pellet burning.

An example of a user add-on to pellet systems: building a burning basket to add to burner or fireplace. Several users made burner basket add-ons that were to be used either inside old oil burners or in the fireplace because some old oil 
burners lack a suitable "fireplace" and fireplaces lack a tray for placing the pellets. Burner baskets can be made from almost any metal thing by bending and cutting. Such an add-on enabled the control of air deflecting in the burning process, a quality that was seen to be lacking in commercial products that were otherwise suited for users' particular conditions.

Examining the user inventions in wood pellet burners reveals that users have also invented in all aspects of this technology. In fact, only the radiators inside the house received no alterations. The Finnish wood pellet burning systems feature two new-to-the-world designs, while the remaining 65 inventive user projects created systems or modifications that recreated the existing types of equipment from metal parts that happened to be available or modified extant equipment (e.g., oil-burning stoves) for wood pellet use.

As with heat pumps internet discussion forums played a major role in the dissemination of ideas and peer assistance, as well as cost reduction appearing to play a role as self-building can produce a working pellet system for $10 \%$ of the price of purchasing a commercial one. Again, we also find local conditions, means, and resources being utilized. However, in relation to this lies a difference between wood pellet and heat-pump projects. Most pellet systems are based on direct engagement with the materials at hand and on-site (i.e., they are not mediated by accurate blueprints or design tools and their distribution over the internet) and consumers create the technology from available materials (cf. Usenyuk et al. 2016), not building on commercially available systems, as was the case in the heat-pump projects.

\subsubsection{Expert evaluations of consumer inventions}

To improve our understanding of the novelty and value of the solutions and the technical difficulty of making them, we proceeded to subject all the innovations (from the above heat pump and pellet burner data, supplemented with solar PV and solar heat inventions - altogether 214 inventions) to expert evaluations, wherein we used four aspects that could be estimated based on the data available on all the consumer innovations in the data set. The first aspect evaluated was inventiveness: how new to the world each innovation was and how radical they were technically. The second aspect was the ease of implementation for potential adopters. The third item in the evaluations was the diffusion potential of the inventions in the total Finnish consumer base: to how large a proportion of the Finnish consumer base could the invention be potentially beneficial and applicable? The experts took the market size as the consumer base into which the modification can proliferate rather than, for example, evaluating the sales potential of the improved model.

The final item for evaluation was energy-saving potential, a proxy for indisputable economic benefit. Here the expert evaluations proved less helpful than in the other categories. For wood pellet burning systems it became too hard to reliably assess the energy saving achieved without field trials. For heat pumps this was easier as, in most cases, there was already a model upon which the 
invention or modification was built. However, over $40 \%$ of the consumer inventions were in fact improvements to the usability, control, and monitoring of the systems. In some such cases, our evaluators provided estimates of indirect energy saving that were mostly higher than the mean direct energy saving of the evaluated inventions. However, we have discarded all but the estimates of indisputable direct savings in order to err on the conservative side in assessing the potential of the consumer innovations.

Overall the three sets of evaluators provided well-aligned appraisals of the inventions (at most, a difference of one degree was found in cross-comparisons), and thus the score for each consumer project could be settled by the majority value among evaluator scores.

In Table 3.4 we can see that most of the innovations were relatively incremental (albeit there were a few technically radical, new-to-the-world innovations). However, particularly the heat pump and solar technology inventions were moderately difficult to implement, typically requiring fair technical sophistication from any adopter wishing to copy the invention. Most of these consumer innovations had limited proliferation potential because the majority of the inventions arose from local contingencies or were specific to a particular model (Hyysalo et al., 2013b).

As expert evaluations of user innovation have been critiqued as featuring potential evaluator sentiment bias and also as we wished to gain a clearer view of the climate change mitigation potential of the user innovations, our colleagues in the Finnish Environment Institute calculated life-cycle carbon assessments for the example user innovations (Mattinen et al., 2014). In short, comparing the ground-source to air-convector heat pump (see the vignette above) with direct electricity (DE) and a high quality, off-the-shelf, ASHP show significant improvements in all of Finland's four climate zones; with ASHP roughly halving the energy use from DE, and GSAHP again halving the energy use of ASHP. What also is worth a remark is the proportion of end-oflife greenhouse gases as this equals the total loss of all refrigerants that are potent greenhouse gases remaining less than $10 \%$ of the GHGs resulting from energy use. This calculation was made in response to a counter-argument expressed many times to us by energy system professionals that "amateurs will just spill the refrigerants and do more harm than good for the climate." Our interviews reveal that refrigerant spilling is quite rare, and if it happens, the life-cycle saving far outweighs it.

\subsection{The diffusion of consumer innovation in S-RETs}

To understand the diffusion mechanisms of S-RET consumer innovations, we need to first understand how adopters can learn about the innovations; in other words, we need to understand the interaction arenas through which the originator of an innovation and its potential adopters are in contact (Heiskanen et al., 2014; Johnson et al., 2014; Hyysalo and Usenyuk, 2015). In the Finnish cases, the contacts between innovating consumers and their peers were sometimes 


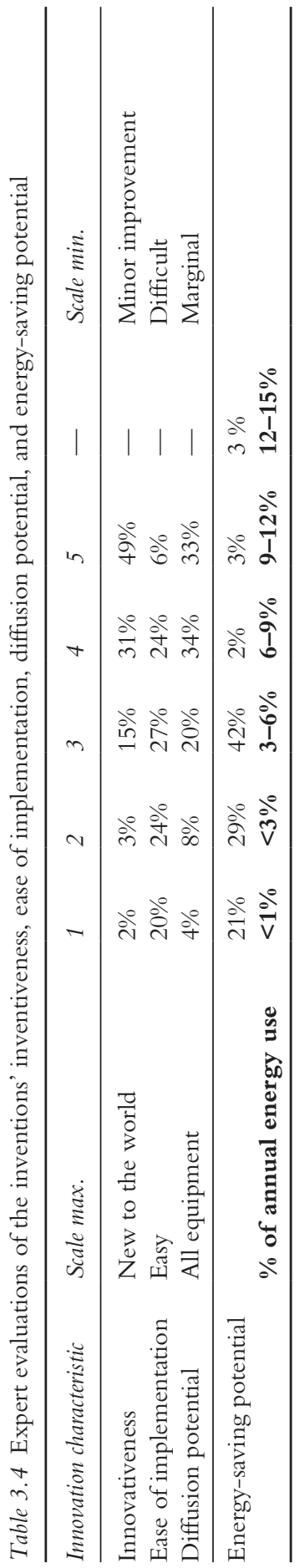


face-to-face but were predominantly mediated by the internet-based discussion forums (Hyysalo et al., 2013a, 2013b).

The innovating consumers mostly used online discussion forums to connect with their geographically dispersed peers during their projects. The form by which they did so followed a "display-and-advice" sequence. They display their projects, ask for advice, and are then provided with comments and suggestions that typically lead to the next display and next round of comments. It is the forum post's author who initiates this, and it is the author's interests that mostly steer the exchanges. The physical character of an S-RET prevents people from directly pooling their work into the same projects as they do in open-source software projects, yet the discussion forums did allow them to solicit problem-solving advice from peers. Regarding diffusion, the displayand-advice sequences tend to create an imperfect documentation trace. They may provide good detail of the innovation project goals, most of the steps the person went through in building it, and the knowledge about what worked and how well. Yet the ensuing documentation trace is not geared toward copying. Not only are innovative solutions not well categorized for adoption (in regard to which model, housing type, or problem they provide solutions for and if the solutions were effective), they are not documented so as to aid adoption: the adopter has to be very interested and often needs to contact the innovator in order to be able to copy the solution as the solution is typically displayed in detail only with respect to aspects that the innovator wished peer commentary.

In the following we examine the types of diffusion paths that were found in the data set. We do this by discussing the original consumer innovation and then proceed to its subsequent diffusion. Empirical studies to date have predominantly examined commercial adaptation: the innovator becoming an entrepreneur (as with Case A below) or revealing an innovation to a company (as with Case B). Our main interest is in how peer diffusion happens (through straight diffusion or innovative adaptation by peers) or does not happen. We thus elaborate on this and give fuller examples of the innovations in Cases C, $\mathrm{D}$, and E.

As discussed in the literature review, a common case of consumer innovation diffusion is the one wherein an innovation originally built for the innovator's own use is further developed by the innovator into a product on the market - in other words, the innovator becomes a consumer entrepreneur (Shah and Tripsas, 2007). In our data set three (3) consumers had developed their designs into a marketable product. The following case, Case A, illustrates this innovation diffusion path.

Case A: The consumer becomes an entrepreneur. The "ground-source air-heat pump" described above is an example of a user becoming an entrepreneur. The inventor had close connections to a small coolant application company, owned by a relative, and he eventually joined the business as a minority owner and the system is now commercially available from Jääsähkö Oy. This company wanted to find a niche solution that would not attract large heat-pump vendors as competitors. In this case the interaction arena for development included various 
knowledge repositories and, most importantly, face-to-face meetings between the kinsmen. The design targets a big $\mathrm{CO}_{2}$ problem in the Finnish housing stock, namely the considerable number of houses $(400,000)$ with direct electric heating, some of them located in regions where ASHPs are not effective.

The second route for consumer innovation diffusion is revealing the innovation to a company, which consumer innovators commonly do (von Hippel, 2005). In our data set we found one case of voluntarily revealing an innovation and one case of it being revealed involuntarily (our Case B here).

Case B: Revealing an innovation to a company. A user ideated a dual-source heat pump, which uses ground-source or air-source heat depending on which one is in a more effective temperature range. The idea was posted in an internet forum and then iterated by several users and adopted, through self-building, by some of them. At some point the original user-designer contacted a Chinese producer of the ASHP he had modified and suggested that they design a dual-source heat pump. The manufacturer responded by apologizing and stating that they could not produce the model and were not interested in pursuing it. However, only a year later another Chinese manufacturer (perhaps not coincidentally from the same city as the one the Finn had contacted) listed exactly the same design on the internet marketplace alibaba.com as its own invention. The interaction arenas here consisted of internet discussion forums, contact between the consumer and manufacturer, and the commercial forum where the innovation became listed.

Case C: The straight adoption of an innovation by peers. The third type of diffusion in our data is the adoption of an innovation by peers through selfbuilding the design of another consumer. The most widespread example of straight adoption by peers was an open license "house logger" program, used to monitor energy solutions in the house. It was coded by a single user who received feedback from peers yet kept the development in his own hands. The open license program was then downloaded, installed, and updated by others. The interaction arena for the adoption and feedback was one of the internet discussion forums.

Straight adoption also happened with physical solutions, such as among the solutions that users created for adjusting the ASHP's ice melting phase. The cooled surface of the heat pump's outdoor unit condenses moisture, which freezes in low temperatures. The machines have a melting sequence to get rid of the cumulated ice, during which time the pump does not heat. A derivative issue about melting the ice is that in below-zero outdoor temperatures, the meltwater must remain melted until it has dripped out of the outdoor unit's box. Most ASHP models have an additional melting coil that is on whenever the outdoor temperature is below zero. The melting sequence and the additional melting coil were common points of user adjustment and innovation as the commercial models were poorly optimized. For instance, in many models the melting coil was on continuously for four months (!), even when it only needed to run for a few minutes every 40-60 minutes. This was reported to waste $500 \mathrm{kWh}$ out of $4500 \mathrm{kWh}$ of ASHPs annual energy use (i.e., over 10\% 
of energy use) in comparison with an optimized system. One solution, copied by several peers without modification, was an additional control to monitor the relative temperature of incoming and outgoing freezer-circuit liquids and thus detect when the melting sequence of the machine was on, switching on and off the melting coil.

As discussed in the literature review on diffusion, not all diffusion takes a straight adoption character. In our data set, innovative diffusion was, in fact, more plentiful than straight adoption, and it came in many varieties (see below). In 26 consumer innovations it was impossible to pinpoint who was the exact originator of a cluster of different consumer solutions for the same or similar problem, which Case D clarifies with one example.

Case D: Diffusion through innovative adaptation by peers. As noted above, several users redesigned ASHPs into ASWHPs. User reports of the most extensive and apparently most prolific project received over 300 replies and 18,000 reads in one of the internet forums. Online community members actively contributed to the design issues faced by user innovator and there were at least tens, if not hundreds, of other users who repeated the design using the posted descriptions.

Over the course of these building efforts, altogether 14 distinct consumer innovations emerged to enhance the converted ASWHPs. All had different parts and sub-configurations, as well as a different 'starting-point' ASHP model. At least three of these consumer designs were copied as they were by several other consumers, but for the other 11 designs there was no evidence of such further copying (though in all likelihood, some of it happened). At some point an initiative for a joint open-hardware project ("the world's best coldclimate air-heat pump") was initiated, but it withered away after several pages of the initial specifications.

In these projects we can differentiate three different types of innovation and diffusion. The most straightforward is where the second adopting consumer adds further features to the first consumer's design upon adopting it. Some adapting consumers also removed unneeded features and, in so doing, ended up making further reconfigurations in order to make the redesign work. Finally, some users ended making a series of changes in adopting the original design, but adopted it nonetheless. For our present discussion, we should bear in mind that these three types of innovation and diffusion are all instances of innovative diffusion if the add-ons or changes in themselves are not novel, but the final outcome differs from the original. (note: if the further changes themselves are novel, the resulting solution would classify as user innovation.) In Figure 3.2 below, these three types of innovation and diffusion are marked as innovative diffusion types D1, D2, and D3. Figure 3.2 clarifies the Cases A-D that show different diffusion paths for innovations by consumers. In all of these cases the interaction arena consisted of the internet discussion forum for displaying projects and comments, and in many cases also of its private messaging functions.

In a further 39 cases, a company solution pre-existed, but consumers had innovated a new solution (not a direct DIY copy) using different materials, eventually resulting in a cheaper price. Case D also clarifies this case as a 
A:

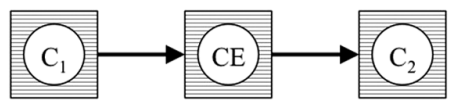

B:

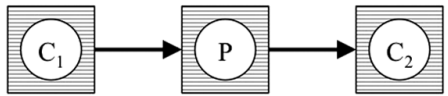

C:

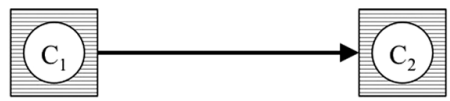

D:

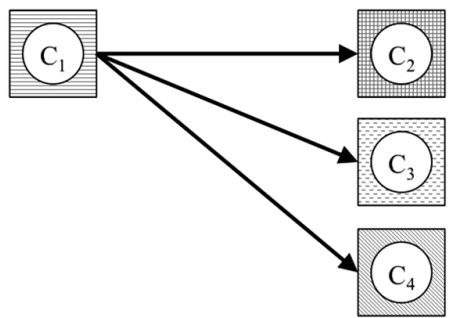

Diffusion through consumer entrepreneur (commercial)

Diffusion through incorporation to existing products (commercial)

Diffusion among peers (adoption as is)

D1: Innovative diffusion among peers (adding new, e.g., adding new features)

D2: Innovative diffusion among peers (partial adaption with subset of features)

D3: Innovative diffusion among peers (modifying, e.g. adapted for different brand/technology platform)

No diffusion.

\begin{tabular}{rlrl}
\hline LEGEND & Actor & $\mathrm{C}=$ Consumer & A change in solution fill pattern \\
$\mathrm{P}$ & $=$ Producer & indicates innovative diffusion. \\
$\mathrm{CE}$ & $=$ Consumer Entrepreneur &
\end{tabular}

Figure 3.2 The diffusion paths of consumer innovations in S-RETs.

different type of ASWHP was commercially available all along. Such innovative peer diffusion solutions could be coded just as validly as user innovations or as (strongly) adaptive adoptions.

Finally, not all consumer innovations had diffusion that could be verified, even in cases when the solution was deemed original and to have potential value for later adopters/adapters by experts who evaluated the potential of consumer innovations for us.

Case E: No diffusion. Consumer innovations can remain underused, although the innovations provide clear benefits. We noticed several solar PV cases that received no diffusion.

A solar panel user noticed that the aluminum frame of solar PV panels froze and the lower rim also began to gather snow, which blocked the sunlight. The user attached a small melting cable, connected to the power grid, to the lower rim of the panels in order to melt the snow. The required heating of the rim necessitates some tens of watts before the panel starts to get sunlight, heats up, and removes the rest of the snow. The yield of these panels on a winter day 
in Finland has been 200-300 Wh, so there is a clear payback. Solar panels are predominantly designed for and assembled in areas where it does not snow. This small consumer addition helps to make use of them in colder conditions. Despite the positive yield and rather easy implementation, there was no evidence that this solution has spread among other users or to manufacturers.

Some user solutions did not spread because they were not actively displayed: for instance, one of the user innovators replaced the above-described melting coil thermostat relay solution (Case C) with a time relay solution synced to the melting cycle and reported higher reliability with it to us (the researchers), yet did not display it to other users.

These diffusion paths found in the material can thus be presented schematically as forming six different paths of diffusion along with no diffusion (Figure 3.2).

When we examine the whole data set regarding diffusion, the 181 projects feature one case that spread through both commercial and non-commercial channels: the dual-source heat-pump project, Case B. As these two diffusion channels are not mutually exclusive, the total number of diffusions examined was 182. As to the extent of diffusion, the businesses of the consumers are either run on the side of their main occupation, or they also sell other services or products. Concerning peer diffusion, the diffusions are hard to track objectively, most projects necessarily remaining unidentified, and it appeared sensible to treat them as less-than-a-hundred diffusions and more-than-a-hundred diffusions in order to match the uncertainty in identifying the diffusions. With this division there is only one case of diffusion that ran to tens of thousands of adopters: the placing of a sledge underneath an outdoor ASHP unit. There are three instances where the verifiable diffusion measured in the hundreds, 17 measuring at least in the tens of diffusions, and in the rest of the cases there were less than ten verifiable instances of diffusion.

The four solutions that have diffused widely merit some description. One the one hand there were two software applications, the "house logger" open license program described above and a spreadsheet software for estimating energy consumption and production, which had an unknown origin and a range of annotations, and was subject to both adaptations and adoptions. On the other hand, there were two physical designs. The first was housing built to cover an outdoor ASHP unit, designed in order to make it fit better with the aesthetics of the house (something that commercial versions did not provide), and the second was the idea of placing a medium-sized plastic box, children's sledge, or some other suitable plastic receptacle underneath an outdoor ASHP unit so that the ice formed and piled under the unit is easy to remove (see Figure 3.1). While these four solutions had a clear value to the consumer, none provided direct energy saving. All of these could also be adopted in almost the full Finnish installation stock, and with the physical designs, adaptive diffusion was easy to accomplish and required no detailed instructions: adopters could use whatever ready-made receptacle they had to hand or build a different housing to match their house. The interaction arenas in these cases were predominantly internet discussion forums. 
Table 3.5 documents the distribution of consumer innovation diffusion. It reveals that commercialization is the smallest subset of diffusion and the cases of innovative diffusion form a class that is four times larger than straight transfer peer diffusion.

The expert evaluations of the user inventions (see the end of Section 3.3) can be further associated with the realized diffusion and linked to existing research on consumer innovation diffusion. Prior user-innovation research on commercial diffusion is relatively established and suggests that the following patterns should take place (Gambardella et al., 2017; Shah and Tripsas, 2007; von Hippel, 2005). Innovations that are patentable, not obvious, and hold widediffusion potential should encourage consumer innovators to seek to commercialize them. If they imply a direct energy saving, this saving should be positively correlated with their commercialization. Concerning the path of the consumer innovation being revealed to and adopted by an outside manufacturer, those that are less innovative and have less diffusion potential are more likely to be revealed. An entrepreneur or production company needs to have the competences and resources to produce an innovation on a commercial scale, and hence the ease or difficulty of implementation should not matter.

For further statistical analysis these interrelations can be formed into four hypotheses:

Hypothesis 1: Consumer innovations that are rated highly for innovativeness are associated with consumers turning into entrepreneurs.

Hypothesis 2: Consumer innovations that are rated highly for diffusion potential are associated with consumers turning into entrepreneurs.

Hypothesis 3: Consumer innovations that are freely revealed to companies are associated with less innovativeness or less diffusion potential.

Hypothesis 4: Consumer innovations that are rated highly for their energysaving potential are associated with consumers turning into entrepreneurs.

We examined commercial diffusion with regard to rated innovativeness, ease of implementation, diffusion potential, and energy saving. Due to the small

Table 3.5 The diffusion of consumer innovations when innovative peer diffusion is included

\begin{tabular}{lcll}
\hline Type of diffusion & Instances & $\%$ & Scale of diffusion \\
\hline $\begin{array}{l}\text { No diffusion } \\
\text { P2P straight peer diffusion }\end{array}$ & 100 & $54.9 \%$ & 0 \\
& 15 & $8.2 \%$ & 1 diffusion $>100$ \\
P2P innovative peer diffusion & 62 & $34.1 \%$ & $\begin{array}{l}14 \text { diffusion }<1-100 \\
\text { diffusions }>100\end{array}$ \\
User entrepreneur & 3 & $1.7 \%$ & $3<$ diffusions $<1-100$ \\
Revealed to the company & 2 & $1.1 \%$ & Not available \\
Total & 182 & $100 \%$ & \\
\hline
\end{tabular}


number of diffusions in our sample, Fisher's test is adequate for evaluating how significantly associated the predictor of innovativeness is for these observed diffusions.

We found that the innovativeness of a consumer innovation is significantly associated with the consumer becoming an entrepreneur, with a significance level below 1\% (see Table 3.6). Similarly, the consumer becoming an entrepreneur is also a function of the diffusion potential $(p=1 \%)$, the ease of implementation ( $p<5 \%)$, and the energy-saving potential $(p<5 \%)$. The consumer innovations commercialized by user turning to entrepreneur were new to the world, and the two innovations revealed to companies were less innovative, in line with Hypotheses 1 and 3. Innovations leading to consumer entrepreneurship were at the difficult end of implementation and held the highest or second-to-highest diffusion potential, unlike those revealed to companies, in line with Hypotheses 2 and 3. With regard to the energy-saving potential (a general value approximation), two out of three consumer entrepreneur cases were for wood pellet burning systems where the estimation of energy saving was impossible for the experts without field trials. The remaining case resides at the second-highest step of the scale, with a saving of $12 \%$, unlike the two innovations revealed to companies, in line with Hypotheses 3 and 4. Overall, the hypotheses are supported: consumer innovations that have diffused commercially stand out as having the potential to do so.

To date, research on the peer diffusion of consumer innovation remains less mature than commercial diffusion, but in light of the research there is (De Jong et al., 2015; Gambardella et al., 2017; von Hippel et al., 2017) innovativeness could have "a cool factor," motivating some technically oriented peers to adopt. But if adopters focus on the costs and benefits, the ease of implementation should be associated with diffusion, as should the higher energy-saving potential of those innovations that directly concern energy saving. Diffusion potential should predict realized diffusion because the population that could adopt it is larger. For statistical analysis these can be expressed as hypotheses on peer diffusion as follows:

Hypothesis 5: Consumer innovations that are rated highly for ease of implementation are associated with peer diffusion.

Table 3.6 The measures of the association between independent and dependent variables through Fisher's exact test

\begin{tabular}{lccll}
\hline & \multicolumn{2}{l}{ Commercial diffusion } & & \\
\cline { 2 - 5 } & "User entrepreneur" & \multicolumn{2}{l}{ "Revealed to comp." } \\
\hline Innovativeness & $\mathbf{2 7 . 3}^{* *}$ & $(.000)$ & 5.1 & $(.454)$ \\
Ease of implementation & $\mathbf{6 . 1}^{*}$ & $(.045)$ & 3.9 & $(.337)$ \\
Diffusion potential & $\mathbf{8 . 8}^{* *}$ & $(.010)$ & 3.6 & $(.501)$ \\
Energy-saving potential & $\mathbf{1 5 . 7}^{*}$ & $(.047)$ & 8.6 & $(.379)$ \\
\hline
\end{tabular}


Hypothesis 6: Consumer innovations that are rated highly for diffusion potential are associated with peer diffusion.

Hypothesis 7: Consumer innovations that concern direct energy saving are associated with peer diffusion.

Since there were more cases of peer diffusion than commercial diffusion, we used the Mann-Whitney $U$ test (a rank-based nonparametric test that can be used to determine if there were differences in the predictor scores between peer diffusion and non-diffusion). We will first examine straight adoption by peers (see Table 3.7). Here the only statistically significant difference was found in the ease of implementation: $U=1674.5, z=2.272, p=0.023$. The ease of implementation scores for straight peer diffusion (mean rank $=119.63$ ) were higher than for no diffusion (mean rank $=88.41$ ); in other words, the more difficult-to-implement projects have diffused, which indicates an opposite association to that stated in Hypothesis 5 .

Next we examine how innovative peer diffusion (see Table 3.8) is associated with evaluations of the consumer innovation's potential in terms of expert evaluations.

Here the relation between the diffusion potential and actual diffusion is significant $(p<0.5)$. Hypothesis 6 gains further support from the cases that have diffused widely. Three out of four were predicted to have diffusion potential

Table 3.7 Mann-Whitney $U$ test results for straight transfer peer diffusion

\begin{tabular}{lccclc}
\hline & \multicolumn{5}{c}{ Straight transfer peer diffusion } \\
\cline { 2 - 6 } & $\mathrm{U}$ & $\mathrm{r}$ & Sig. & Mean no. of diffusions & Mean diffusion \\
\hline Innovativeness & 937.5 & 0.13 & $(0.086)$ & 92.85 & 70.50 \\
$\begin{array}{l}\text { Ease of } \\
\quad \text { implementation }\end{array}$ & $1674.5^{*}$ & $0.17^{*}$ & $(0.023)$ & 88.41 & 119.63 \\
$\begin{array}{l}\text { Diffusion potential } \\
\text { Energy-saving }\end{array}$ & 1244.5 & 0.00 & $(0.998)$ & 91.00 & 90.97 \\
$\quad 291.5$ & 0.03 & $(0.830)$ & 31.28 & 32.50 \\
\hline potential & & & & & \\
\hline
\end{tabular}

Table 3.8 Mann-Whitney $U$ test results for innovative peer diffusion

\begin{tabular}{lcllll}
\hline & \multicolumn{5}{l}{ Peer diffusion: innovative diffusion } \\
\cline { 2 - 6 } & $\mathrm{U}$ & $\mathrm{r}$ & Sig. & $\begin{array}{l}\text { Mean lack of } \\
\text { diffusion }\end{array}$ & $\begin{array}{l}\text { Mean } \\
\text { diffusion }\end{array}$ \\
\hline Innovativeness & 4372.5 & 0.08 & $(0.269)$ & 87.55 & 95.56 \\
Ease of implementation & $5410.5^{* *}$ & $0.30^{* *}$ & $(0.000)$ & 77.47 & 108.87 \\
Diffusion potential & $4820.5^{*}$ & $0.18^{*}$ & $(0.016)$ & 83.20 & 101.30 \\
Energy-saving potential & 513.5 & 0.08 & $(0.538)$ & 29.98 & 32.67 \\
\hline
\end{tabular}


for all equipment on the market. Fisher's exact test's two-sided significance for wide-diffusion items is 0.000 .

The other significant association is between the ease of implementation and realized diffusion ( $p<0.01$; the $r$ value of 0.30 signifies a medium-sized effect). The mean rank scores (108.87 for diffusion and 77.47 for no diffusion) indicate that more difficult-to-implement projects have diffused than easy-to-implement projects. However, when we examine those projects that have diffused wider than 100 verified instances of diffusion, we find they are all at the easiest level of implementation. We interpret this as follows. The rational diffusion predictor for peer diffusion explains the wide adoption (Hypothesis 5). More limited adoption predominantly takes place among technically competent consumers who are not hindered by the difficulty of implementation and in fact may view the technical challenge positively, that is to say as a "process benefit" (Franke and Schreier, 2010).

General economic benefits (in our data, measured by direct energy-saving potential) were not associated with peer diffusion (Hypothesis 7), a finding that parallels the survey of De Jong et al. (2015) on the general Finnish population innovating in any consumer product category.

\subsection{Chapter discussion: user innovation in S-RETs and their diffusion}

Unlike assumed by the mainstream of energy innovation research, citizen users hold considerable ability to modify and invent in their home energy technologies. Over 200 user inventions, designs, modifications, and add-ons could be identified in the Finnish context alone. Our analyses show that users were able to invent in practically all parts and subsystems of the S-RET technologies. The subsystems that did not feature user modifications, such as compressors and radiators, appeared to be parts that users did not find a need to tinker with rather than those that were too difficult to modify.

To understand why citizen users choose to innovate, an analysis of the content of their innovations is instructive. In the first instance, user inventions point at what is missing in the market: These consumers become aware of the limitations of the technology and try to solve the problems by inventing (cf. von Hippel, 1988). Most user inventors built solutions that had one or several facets that would have rendered them unsuitable for the mass producers of the systems. The inventions were either useful for relatively specific locales, compromised the durability or reliability of the system in a manner that could be locally compensated for but would not suit all users, or combined different technologies in a manner that commercial manufacturers would not find appealing, even when local benefits were obvious. In this capacity user invention presents a complementary direction for the advancement of technology to research- or manufacturer-driven $\mathrm{R} \& \mathrm{D}$. The inventors were aware of the risks their experimental projects could entail and, judging from the forum discussions, had created functional local arrangements to handle the risks. This 


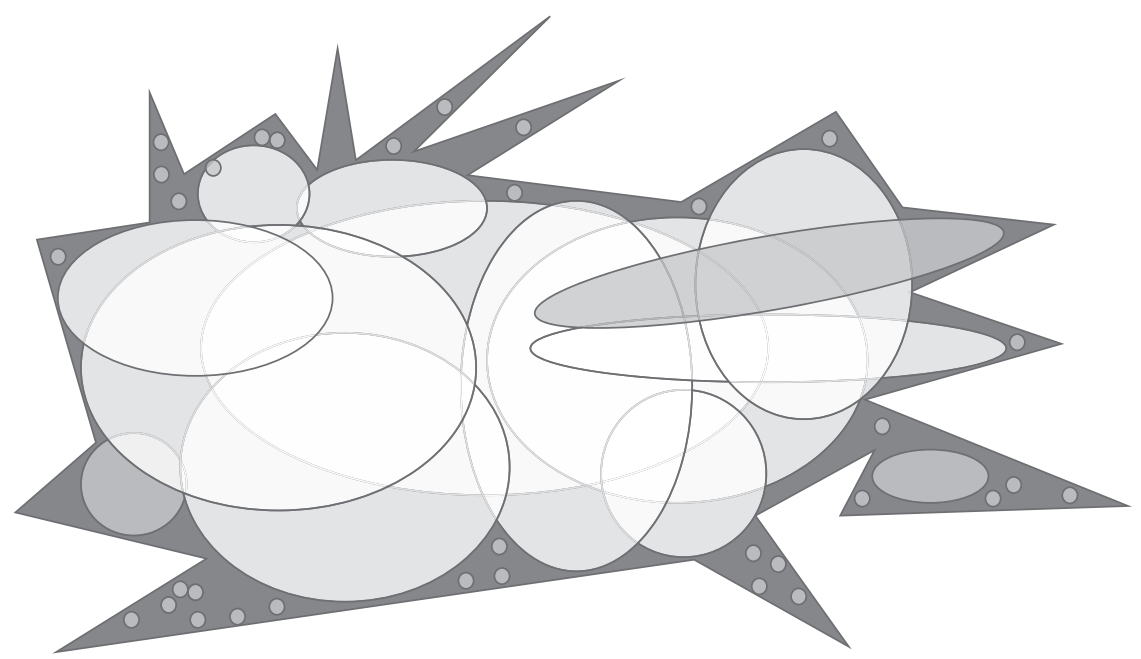

Figure 3.3 A schematic illustration of design spaces under conditions of high variation in user needs.

dynamic is schematically illustrated in Figure 3.3 through depicting the interrelations between design spaces that feature local particularities that invite user innovations as commercial offerings only seek to appeal to larger sets of clientele to recoup investments and in doing so leave aspects of design space uncatered for. In Figure 3.3. the user needs and preferences (dark background) form a space in which majority of needs are shared by many customers and can thus be profitably met by commercial solutions (white circles) targeted at different customer segments. Even as the market matures, the design space continues to feature need spaces that are too particular for profitable commercial products (dark corners) and the unserved users with these needs continue to innovate to serve themselves (light grey circles), with some of their innovations growing into products appropriated by the wider customer base as well.

Taken together, these findings indicate that some energy users have significant competence and the will to have a deep and active relationship with their energy technologies. The rise, spread, and visibility of sophisticated DIY competences and projects have been facilitated by user-run internet forums that allow peer help and thus the mixing of professional competences that are often requisites for user inventors to achieve their designs. As with most other technologies, the making of inventive modifications is concentrated on a few individuals (von Hippel, 2005).

Regarding diffusion, the next chapters of this book elaborate how innovating consumers have aided the overall diffusion of renewables in Finland through peer assistance on online forums. The spread of consumer solutions themselves shows that only three cases $(1.7 \%$ of those studied) led to the 
consumer becoming an entrepreneur and two (1.1\%) were revealed to companies. Widespread $(>100)$, verifiable diffusion among peers took place in four cases $(2.2 \%)$. Thus, the direct impact of solution diffusion appears somewhat limited.

The reason may well lie in that few projects were accompanied with detailed instructions concerning how to replicate them- they were thus poorly observable and could not be trialed without extensive effort (Rogers, 2010). Innovations that diffused widely were in the easiest category of implementation and in the widest category of diffusion potential. For such simple-to-replicate solutions, the work of exhibiting them on online forums would be sufficient to spur their wide diffusion. In contrast, modest-scale diffusion occurred in 71 of the 181 projects $(39.2 \%)$, mostly through innovative peer diffusion. This is likely to be no coincidence. Innovating consumers only leaving a displayand-advice trace is in line with previous findings on how user innovators lack incentives to further the adoption of their solutions (von Hippel et al., 2017). However, the low diffusion of innovations that have been evaluated by experts as being socially valuable could be a market failure in terms of welfare economics (Gambardella et al., 2017; von Hippel et al., 2017).

Our data underscores how equating the diffusion of consumer innovations with adoption, or in other words straight peer diffusion, may require reconsideration. In classic diffusion studies, the diffused item was taken for granted-usually a discreet item such as a new agricultural seed type or a simple tool introduced by a commercial entity or change agency (Rogers, 2010). In contrast, S-RETs are at least moderately complex technical configurations. In our data set the largest type of diffusion was innovative peer diffusion, where different consumers continued to incrementally and iteratively adapt the solution they adopted. This results in materially and conceptually distinct consumer solutions that influence each other. This phenomenon is known to previous research. Adaptive diffusion, innofusion, innovation at adoption, design-in-use, and the fluid deployment of technology are terms used to describe variations of innovative technology uptake (Agarwal, 1983; De Laet and Mol, 2000; Douthwaite et al., 2001; Fleck, 1993b; Hyysalo and Usenyuk, 2015; Williams et al., 2005; Kohtala et al., 2020).

The high incidence of innovative peer diffusion in our data set has not previously been recognized by the user-innovation literature but makes sense in the context of it. Its basic premise is that users innovate for themselves because each consumer has a somewhat different set of competences, preferences, and contexts of use, and also some consumers remain underserved by the offers available on the market (von Hippel, 2005, 2016). It is cost-effective for users to pool their competences if communication and design costs are low enough, which can be achieved through computer-mediated communication and modular design architectures (Baldwin and von Hippel, 2011). After all, if users hold roughly analogous needs it would be wasteful for each of them to seek to independently invent the same solution (von Hippel, 2005).

However, the consumers examined in the present study consume goods in geographically and socially differentiated contexts and are united by digital 
forums that are only geared toward discussions. The nature of their projects and their interaction arenas allow them to enjoy some pooling gains, but lack others:

$+\quad$ They can gain information on which solutions have and which have not worked for their peers and thus focus their own solution search and reduce the (often considerable) costs of trialing entirely untested solutions.

+ They can gain solution outlines and advice in regard to realizing relatedsolution search directions from their peers.

- They have a somewhat different technical configuration with sunk investments (such as the heating equipment and house) and thus direct copying may not make sense for them.

- They cannot assess the quality of another user's solution and its fit and value for themselves by just running it (as in software) - they need to build it up and fit it before they can verify its value, which may pose a barrier to adoption.

- They are likely to be unable to each build a module and fit it into an architecture that benefits them all; hence their complementary output cannot add up in a manner similar to using software, even if organized.

- They do not have a way to share instructions with other users without the extra effort of making such instructions (see the above section on under-diffusion).

With these possibilities and limitations, it becomes sensible for consumers to pursue innovative peer diffusion in order to satisfy their unmet needs. The risk that organized community collaboration would not pay back is high, yet seeking independent solutions in "packs" offers them some of the innovation community benefits. The findings thus raise a question for further research with regard to whether studies of the peer diffusion of physical products should expect two qualitatively different peer diffusion pathways: one of adoption and one of adaptive innovative diffusion. The latter is likely to remain the prevalent case for S-RET peer diffusion as S-RETs will remain physical technologies in the future.

To sum up this chapter, citizen consumers display considerable ability and willingness to innovate in S-RETs. The Finnish cases underscore how active consumer roles remain important after the formative stages of S-RETs as they proliferate in new country contexts, markets, and institutional settings (Juntunen, 2014b; Heiskanen et al., 2014) where the standard commercial S-RET solutions may not optimally fit upon their market entry. Consumer innovators of S-RETs add to the solution variety of the S-RETs available for other adopters. It is worth remembering that the accumulation of minor improvements is responsible for a major part of technological progress (Rosenberg, 1979). However, our study indicates that the direct solution spread remains limited, apart from a handful of solutions. This is a disappointing finding for those who envision consumer innovations as a direct additional 
means for energy and climate policy to boost S-RET solution development: the solutions add variety rather more in principle than in practice.

The other item we clarified is that the low diffusion of innovations by consumers does not appear to be an issue of solution quality, as domain experts assess many of the innovations to be socially valuable, and their assessments predict well which solutions lead to commercialization. The rest of the solutions, in principle freely available for peer-to-peer adoption, suffer from not being rendered in an easily adoptable form. These solutions are free but not easy to replicate, even after peers have become aware of them. As a consequence, peer adoption happens more through innovative peer diffusion, yet this form of adoption requires higher competence and active engagement with technology in contrast to adopting a well-documented solution as it stands. At the same time, inventing and innovative diffusion fosters competence development among the consumers who pursue it. As we will discuss in the next chapters, the users peer-to-peer interactions and competence building may eventually be more important for the overall shaping of sociotechnical change than user innovation.

Our results underscore that it would be a mistake to isolate the significance of each user innovation and user modification, and only consider their straight adoption without considering how they feed into further projects. Thus, the formed pathways of user improvements lead to the articulation of demand for improving the commercial systems for new market areas. The managerial implication is that on entry to new markets, producers would benefit from following the interaction arenas where users discuss the technology and display their problems and solutions, thus revealing the points that need improvement in the generic technology for the market particularities.

The ensuing policy implication is that peer support among consumers is a valuable resource that should not be accidentally curbed through, for instance, measures that would affect their anonymity or any of the other self-organizing principles by which they thrive.

What could be done to counter the potential under-diffusion of consumers' innovations in S-RETs? The solutions that exist to date, such as platforms for physically making things in the form of parts lists and recipe-like texts (as found at instructables.com), are unlikely to work as solutions. Following the reasoning of von Hippel et al. (2017), few innovating consumers would have the incentive to take the time and energy to document their often complex hacks and designs. The innovating consumers could, however, be motivated to do so if there were templates available for the effective display of their problems in such a way that it would help to present them and, as a side effect, make a more structured information package out of the display (e.g., if an easy way to make blueprints was available or if there was an easy way for the user to point to the exact location of the modification and make parts lists). Our findings on innovative diffusion thus call for further technology development in the platforms for physically sharing designs among peers. 
From the BOAP methodological perspective, the studies on user innovation show a string pattern that ensued after a surprisingly large number of innovations found in the initial study. It became interesting to better understand their nature, social value, the competences needed to make them, and their diffusion, thus producing a string of studies with complementary foci to zooming in interesting issues. In the discussions of the original articles, one can observe that diffusion in particular was assumed to be much higher and to be concentrated on easy-to-make solutions and commercialized solutions-both assumptions that proved untenable on closer analysis. Moreover, research foci increasingly shifted from the innovations to the competencies leading to them and the peer-to-peer knowledge creation in user communities that gave rise to these competencies. This is what we will examine next in Chapter 4.

\section{Notes}

1 This chapter integrates and expands research that has previously appeared in "Hyysalo et al. (2013a)" and "Hyysalo et al. (2017)."

2 http://lampopumput.info/foorumi/index.php?topic $=1279.0$

$3 \mathrm{http} / / /$ lampopumput.info/foorumi/index.php?topic $=7482.30$

4 http://kotisivu.dnainternet.net/juslaat2/innovaatio.html

5 http://lampopumput.info/foorumi/index.php?topic $=2185.0$

6 http://www.pellettikeskustelu.net/keskustelu/viewtopic.php?f=7\&t=2618 


\section{Broadening the inquiry \\ New Internet-based energy communities}

\subsection{Introduction: communities as catalysts for innovation and technology proliferation ${ }^{1}$}

The importance of internet-enabled and user-maintained forums became salient in the course of our ethnographic data gathering. These online environments allow dispersed energy users to seek information and tap into each other's ideas in a manner that far exceeds anything that adopters, users, and self-builders have had at their disposal in the past. While internet communities have existed for some decades, they have began to provide widespread support for different technologies only after the introduction of easy-to-use opensource platform software in early 2000s, after which it took some time for the communities to mature.

As such peer-to-peer exchanges have long been known to be important for the dissemination of new ideas and novel technologies (Rogers, 2010). Peers are further known to aid in the selection, appropriation, integration, and adaptation of new technologies (see, e.g., Bakardjieva, 2005; Stewart, 2003) as "local experts" (Stewart, 2003; Bakardjieva, 2005) and "configurers" of generic solutions to the specific needs of other adopters (Okamura et al., 1994; Barnes, 2016). In these capacities, peers act as user-side intermediaries that are involved in configuring technologies, users, and spaces for technology appropriation; facilitating the uptake of new technologies; and brokering connections and transactions between the other actors involved (Stewart and Hyysalo, 2008; Hakkarainen and Hyysalo, 2016; Barnes, 2016).

Also innovating users tend to get help from their peers, and this help is often vital for realizing their designs (Lüthje et al. 2005; Jeppesen and Molin, 2003). As in more conventional $\mathrm{R} \& \mathrm{D}$, specialization is high and inventive users are proficient in particular aspects of focal technology, and the scope of design they can master alone remains more limited than when pooling their competencies with peers (Baldwin and von Hippel, 2011; Benkler, 2006). In many domains, innovating users are deeply embedded in communities of practice and have organized design-oriented networks within them (Franke and Shah, 2003; Baldwin et al., 2006; Hienerth, 2006; Bethwaite, 2008). Also in renewable energy the few reported user-innovation success stories feature self-building

DOI: $10.4324 / 9781003133919$ 
groups and cooperatives (Ornetzeder and Rohracher, 2006; Seyfang, 2010; Nielsen, 2016), and this may not be just because of their higher visibility to researchers.

However, users (particularly the relatively few innovation-prone ones) are often geographically dispersed (von Hippel, 2005). Internet-enabled services can boost visibility and connectivity among people who share the same interests. This has been observed in many online game environments (Jeppesen and Molin, 2003; Prügl and Schreier, 2006), open-source development (Freeman, 2011; Benkler, 2006), and variations of the open design movement (Abell et al., 2011; Kohtala, 2017), and also in designing physical products, even if only some aspects of these products could be shared across the web (Sawhney et al., 2005; Jeppesen and Frederiksen, 2006). Studies of online peer innovation support have, however, examined relatively uniform hobbyist communities of practice and have mostly done so quantitatively (Jeppesen and Molin, 2003; Jeppesen and Fredrikssen, 2006). There is some research on DIY modifications and the related blogs and forums, and how they act as information repositories (Shove et al., 2007; Kuznetsov and Paulos, 2010) and showcase how personal experiences and knowledge gained from creating physical objects are projected into the public sphere (Kuznetsov and Paulos, 2010; Meelen et al., 2019). Nevertheless, there is little research on the interrelation between user-innovation and online user forums that are not set up for innovation per se. User-run internet communities are, at most, pointed to as a resource for managers to harvest or mine innovative ideas for manufacturers' benefit (Jeppesen and Molin, 2003; Pitta and Fowler, 2005; Franke et al., 2006). This is an important oversight in research given that user-run discussion forums are the largest type of product- and technologyrelated internet communities and the most widespread in terms of the topical areas they cover. In such forums, people organize themselves around products and technologies to discuss use, purchasing, experiences, community knowledge about products and producers, and DIY projects, etc.

To go deeper into the role that user-run internet forums play in adoption and user inventiveness, we turn to the concepts of science and technology studies (S\&TS) as a means to describe participation in complex sociotechnical formations. Our first point of departure is that design and use are not static categories and should be examined as emerging and evolving in their relationship to technology (Hyysalo, 2010; Johnson et al., 2010). People are not born users, lay users, or lead users, but rather, the emergence and evolution of their usership should be examined as a processual engagement between the person, the practices she or he enacts, and the technology she or he appropriates (Helgesson and Kjellberg, 2009). In such an engagement process, peers and peer networks have been observed to play a significant role as user-side intermediaries (Stewart and Hyysalo, 2008) that address the often mundane, but important, assistance that goes into the purchasing process and making technologies work.

The processual view endorsed by S\&TS further underscores that attention should be paid to more than just giving and receiving help and contributions, which dominates user-innovation studies on the topic. Many user communities 
(e.g., local communities, issue-based digital communities, the user-developer communities of software and hardware) are known to be multifunctional spaces for their participants (Freeman, 2007; Grabher and Ibert, 2014; Johnson et al., 2010; Mozaffar, 2016; Smith et al., 2016a, 2016b; Verhaeg et al., 2016). Attention needs thus to be broadened to different orientations and learning that takes place in the course of the development of usership (i.e., the competences the participants build and to what effect) (Heiskanen et al., 2010). Concepts that seek to capture learning as participation such as legitimate peripheral participation are particularly helpful as they help capture the often complex and multifaceted learning (and growth) process of a person moving from being a relative novice towards having increasing mastery of a given practice (Lave and Wenger, 1991; Wenger, 1998).

The further pertinent questions raised by S\&TS concern how the practices in a given type of internet community are organized. Here, internet forums feature considerable diversity. Some such forums accompany a relatively homogeneous practice, such as the forum for single-speed bicycles, which could well be characterized as a medium for a community of practice (Wenger, 1998). Others are tied to a joint development project, such as is the case in most open-source development initiatives, rendering such community primarily an innovation community (Jeppesen and Frederiksen, 2006; Freeman, 2011; Heiskanen et al., 2010). Yet other forums are diverse to the extreme, such America Online (AOL) or Suomi24 (Finland24), which feature thousands of discussion areas and interests, and little moderation, making most of the discussions threads too low in competence and reliability to aid technology adoption, or for making modifications or reliably qualifying products (Grapher and Ibert, 2014).

In between these extremes are product- and technology-focused-and typically user-moderated-internet discussion forums that exist for many product, software, and technology types. They feature broader sets of orientations than a community of practice would but are focalized, moderated, and curated (through pinning important posts, moving posts to appropriate sections, linking related posts, and so on) nonetheless. Such forums can be characterized as digital boundary infrastructures that allow for a partial co-existence of multiple social worlds by catering for the wide and accessible entry of many participants while providing various sections of deep knowledge and engagement for those with the interest and competency to engage in them (Bowker and Star, 1999; Johnson et al., 2010).

All these internet community/collectivity forms entail somewhat different modes of participation and learning. The user-run internet forums on renewables that we research enable learning through legitimate peripheral participation but appear to be more diverse in their participants' orientations, competences, and interests than, say, sporting-related communities or project-oriented innovation communities. This is also visible in the outcomes of the projects of their participants, which range from simple adoption and adaptations on to monitoring, modifying, inventing, and acting on the market.

The diversity and geographic spread of usership also make the internet communities novel regarding sustainable energy. Research on the early 
phases of energy transition underscores the importance of citizen groups, such as community energy groups, for example, working as activists and innovators, initiating the development of novel S-RETs (Ornezeder and Rohracher, 2006, 2013; Nielsen, 2016). Research on community energy and grassroots innovation (e.g., Smith et al., 2014, 2016b; Walker and DevineWright, 2008; Hargreaves et al., 2013) and energy-related citizen movements (e.g., Ornetzeder and Rohracher, 2006, 2013; Nielsen, 2016) has further underscored how communities and movements create solutions that can be adopted into the mainstream, inflict change among dominant regime actors, and foster critical discourse and the practicing of technological and social alternatives (Smith et al., 2016). Following this, the energy-related research on communities has to date largely concentrated on community groups and movements that are united by an ideological commitment to alternative forms of energy and are often also geographically local (see, e.g., Devine-Wright and Walker, 2008; Smith et al., 2016b). Community energy groups have also co-operated with each other through shared learning and networking, often facilitated by national and regional intermediaries and recently also via emerging international platforms (Smith et al., 2016b; Alarcón Ferrari and Chartier, 2017; Hyysalo et al., 2018). In some countries such as Denmark and Germany energy cooperatives have become recognized energy market actors, and this is likely to become amplified with the new EU energy community directive (Ehnert et al., 2017; Romero-Rubio and de Andrés Díaz, 2015). In all, community energy initiatives have been important in the early phases of energy transition, but it remains open as to what the traditional locality-based communities and new digitally mediated communities offer for the mass-market phase of sociotechnical change. Below we seek to clarify their differences and complementarities.

Our journey into S-RET-related internet communities proceeds as follows. We first examine what internet communities offer for individual citizen users who seek to acquire or deepen their understanding of S-RET. After this we discuss the forums as more aggregated phenomena and discuss how they affect systems change. We end by discussing the implications of their potential for wide diffusion (aka the "acceleration phase of transition"; Rotmans et al., 2001) by comparing their structure to locality-bound community energy forms and distributed cooperatives, as well as discussing the nature of the technology-related discourse these different forums tend to foster. In the final section we draw conclusions.

\subsection{The individual's view: from acquiring usership to growing inventive}

\subsubsection{Supporting acquisition and usership: Providing the information missing in the market}

The bulk of the 500,000 posts in the heat-pump forums deal with issues that have the most relevance to people who are considering whether to buy a heat 
pump, which model would be most suitable, and how to handle typical acquisition problems. The posts are well categorized so as to facilitate comparisons and finding information: general discussion, brand-specific discussions, and usage stories, in addition to sections on the acquisition, scaling, and ordering of heat pumps.

Let us first examine in detail a key "entry post" on ASHPs from 2007 (see Figure 4.1), pinned as "read this first" in the acquisitions section.

The post shows a condensation of much of the knowledge that prospective adopters need to understand and which would be difficult for them to establish otherwise. The first five bullet points deal with technical issues and introduce the relevant terminology: what solutions can be regarded as up to date and the threshold values for typical concerns such as noise level. These are followed by three bullets for the Finnish national context on how to scale the heat pump for different winter temperatures. The most important issues feature links to other posts that provide more information at the forum. The lower list of bullets instructs how to navigate vendor-provided information. Here the peer perspective becomes visible through an instruction regarding the two core values (the coefficient of performance and max power) which run in opposition and how vendor-provided information obfuscates this, particularly for cold conditions.

Resulting in 40,000 reads in five years, this posting and many of its kind help potential buyers to approximate what they need to know in order to make a sensible heat-pump acquisition: clarifying the characteristics of the space to be heated; the energy use and cost profile; the current heating system and whether it makes sense to replace it or combine it with S-RET solutions, such as pellet burner or solar PV; the actual local weather conditions particularly during winter; considering heating controls and operation options such as set-timers and remote operation; and personal preferences on what the heat pump may sound and look like post-installation. Guiding prospective users to these parameters is paired with instructions, calculators, and long threads related to each of these key topics. For instance, with respect to local weather conditions, winter temperatures are linked to (1) energy consumption data in different locations, gained from the research institutes and users providing their actual monitoring data and (2) particular heat pump makes and models, and their measured efficiency for optimizing the investment and regarding the possibilities to retain some of the existing heating options (e.g., solid wood or oil heating) ${ }^{2}$ (Hyysalo et al., 2018).

In doing so forums provide a suggestive image of what it means to be an informed consumer of S-RET and the nature of the information to be consumed. In Stewart's words, they preconfigure the user regarding the relevance of technical characteristics (Stewart, 2003; Hyysalo, 2010). The entry postings typically collect, condense, integrate, and explain information produced by other parties. They further configure the information to the national market specifics, making it directly relevant and reducing scientific and professional content that is not directly relevant for peers. Such bricolage, aggregating, editing, and 


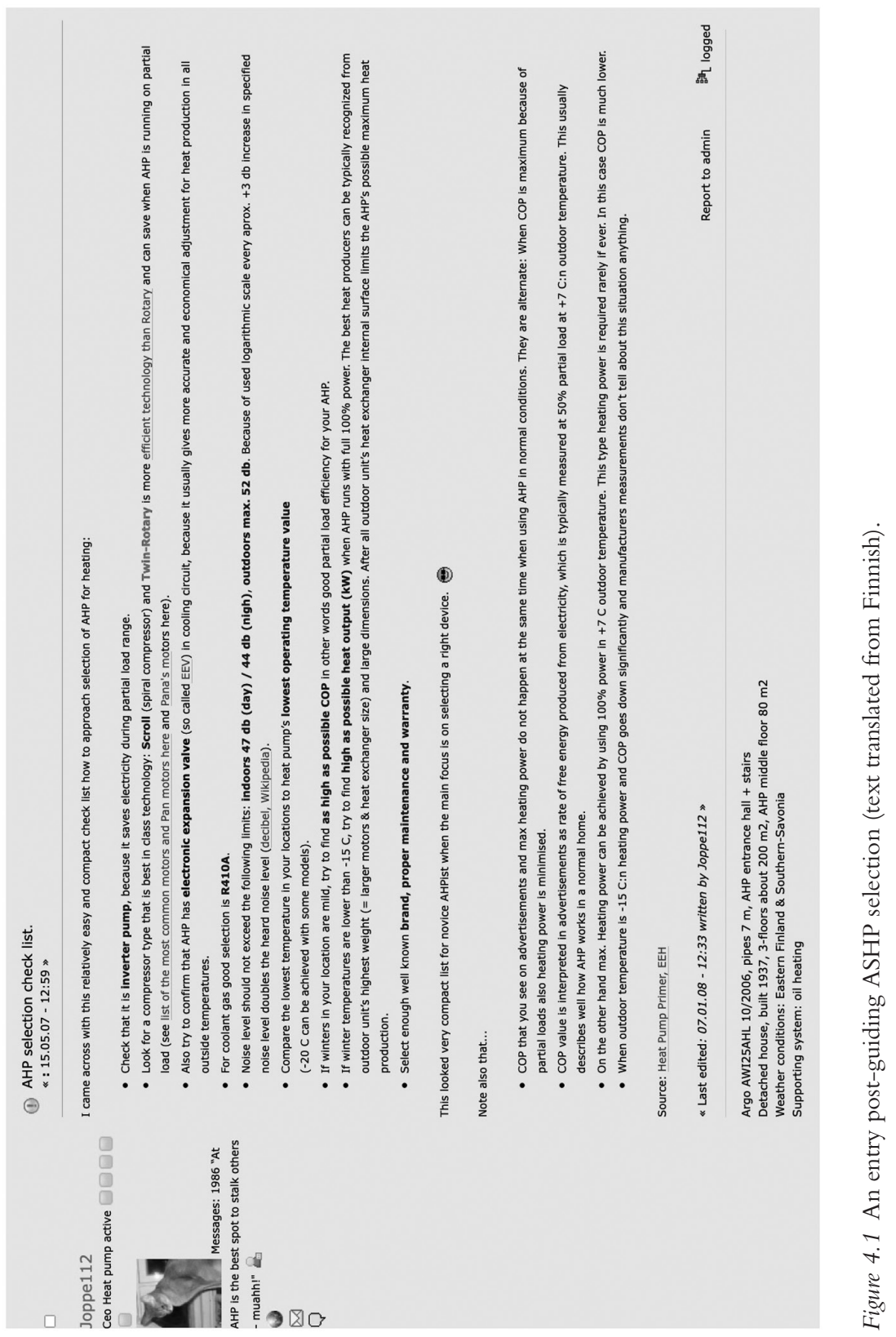


opening topics (Botero, 2013; Botero and Hyysalo, 2013) for peer commentary and validation are typical infrastructuring activities of peers (Pipek and Wulf, 2009), which greatly facilitates other users even though peers do not physically configure each other's energy equipment.

Somewhat more advanced posts establish the relation between particular concrete projects and the principles of sound action in the market. These also give an idea of the magnitude of effort that may be involved in acquisition (and thus the service the forums provide for the readers); let us briefly recount a calculation posted by one of the users (Figure 4.2) to aid others in the task

\section{(-) Mitsubishi FA25V Row House 96 m2 Hyvinkää \\ «: $31.01 .07-: 19: 18 »$}

Scaling the Air-Source Heat Pump into a new house with no prior knowledge or experience of consumption is problematic. Below my own attempts at the subject matter.

http://lampopumput.info/foorumi/index.php/topic,1334.0.html http://lampopumput.info/foorumi/index.php/topic,1010. msg9643.html

The problem of choosing the 'right' pump came after this, once the 'right' size has been defined.

http://lampopumput.info/foorumi/index.php/topic, $986.0 . \mathrm{html}$

http://lampopumput.info/foorumi/index.php/topic,1409.0.html

Simultaneously while asking for offers on equipment, one has to, of course, plan where to locate the equipment and rack in the apartment.

http://lampopumput.info/foorumi/index.php/topic, 1052.0.html

http://lampopumput.info/foorumi/index.php/topic,1711.msg18519.html\#msg18519

It took me 5 months to do the above mentioned, but I could not move into the new apartment before that anyway.

Row house 4 rooms + Kitchen + Sauna heating_power need.png

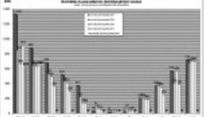

Standardized heating electricity need.jpg

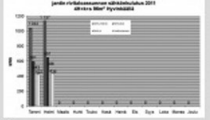

Monthly electricity consumption 2011.jpg

Home COP Test Mitsu FD35VABH.xls

« Edited: 28.02.11 - klo:15:53 Written by N.N »

Row house 4 rooms + Kitchen + Sauna 96 m2 Mitsubishi Electric FD35VABH Hyvinkää and log data N.N.(at)lampopumput.info

Figure 4.2 An example of a forum user giving advice for others regarding scaling and selecting a heat pump, and finding a location for it (translated from Finnish). 
of scaling and choosing a heat pump for a newly built house that lacks any energy-use history upon which to draw.

There are several issues in this posting worthy of our attention. It reports a necessary task (scaling and choosing a model) that new users must engage in. One could assume this to be an easy task, or at least one easily available from vendors or suppliers, but the post points to the difficulties involved: it took five months to do it properly and reliably for a seasoned person when reference data was missing. Without it, any supplier or assembler advice would be hard to assess (their quotes potentially having self-serving biases). It also indicates that a one- to three-hour assessment by a supplier would be a rather rough estimate at best. The uptake of the post, read almost 100,000 times, bears witness to the demand for this type of information and calculation model.

Finally, the signature of the posting is typical and telling of the forum sociality. Instead of a name or some information about the person (e.g., her or his interests or education), there is a description of his house, its location, and the heat-pump equipment in use. His signature tells readers what he has done with heat pumps (consumption monitoring, ASHP scaling, and ASHP pictures); all in all, it articulates the parameters that another user can use to qualify the provided information and comparing the setting with her or his own. The signature is not information about the "discussant" or "person" but about a specific relationship (between a person, equipment, and the context) and specific relational sociality between those engaged or engaging in similar technology and contextual relations. To paraphrase Helgesson and Kjellberg (2009), these forum posts foster the emergence and deepening of usership, the relation between people and the technologies they are engaged in, and interestingly, in the whole forum one finds only isolated items that broaden the writer's position beyond that of his or her technological relationships.

In these relationships, the forum helps make what Stewart and Hyysalo (2008) call user-side intermediation available to peers. For instance, the specific pump-type discussion features peers who are a little more knowledgeable about the technology and are happy to reply to questions about the technology that may appear silly - a noted core facet in the uptake of any more complex system (Sørensen and Williams, 2002; Berker et al., 2006). Forums also feature local experts (Stewart, 2003, 2007), people who are more knowledgeable than most users and to whom more tricky problems can be addressed. Some of the local experts are just seasoned users, but also professional assemblers, resellers, and dedicated hobbyists volunteer their advice regularly on the forums once an issue in a thread becomes flagged as interesting for high-competence people and requiring more serious thought.

As noted regarding the entry post, a key aspect of these intermediary actions is to point to non-human mediators by providing pointers to previous threads on the same topic, links to manuals, instruction videos on YouTube, web pages elsewhere, and to the coefficient of performance calculators. While most users are quite able to follow instructions, the step toward defining the problem 
or question is the part where more knowledgeable peers become indispensable. As one of the user inventors reflects:

After all, the forums provided a lot of tips before I acquired [a heat pump]. [I] browsed and read much about what brands would be worth ordering.

(User inventor for AHPs)

All in all, acquisitions, scaling, help with problems, and help with dealing with suppliers are topics that draw in thousands of people. This provides a critical mass of people with the potential to answer complex and difficult questions and allows for timely and competent feedback. We shall next argue that it lays the ground for deepening orientations and actions related to the S-RETs.

\subsubsection{Learning technology and deepening engagement}

Several of our interviewees stressed that the forum led them into having a deeper engagement with both the knowledge base and with what can be done with the technology. Let us illustrate this with forum members' comments at different stages of their participation, moving from meeting problems in use toward DIY projects and finally turning to lead users:

Thanks for the clear information. In this area [a ground circuit] it feels that when one grasps one thing, you just end up with further questions.

(User, GSHP forum)

Without this forum I would have faced huge problems with this unit. I doubt that I would have proceeded building this further. It [the forum] has been of great help.

(Inventing user for ASWHPs)

I have rather given more to the forum than taken from it. That's the direction ... I have tried to instruct guys who do this [DIY project], especially in dimensioning, so that they get it working and one does not go too far astray.

(Semi-professional who creates designs for a small reseller)

An important step in the deepening of learning lies in moving from just implementing a heat pump to understanding how it works and what issues affect its functioning. This typically ensues from gathering, organizing, and sharing comparative usage data. In the forums hundreds of people post or automatically feed their measurements into the forum for comparison and comments or to answer polls set by others (for example, see Figure 4.3). Most of these users are out to verify how their installation compares to others and to factory claims. Some have more general interests in establishing how different models 

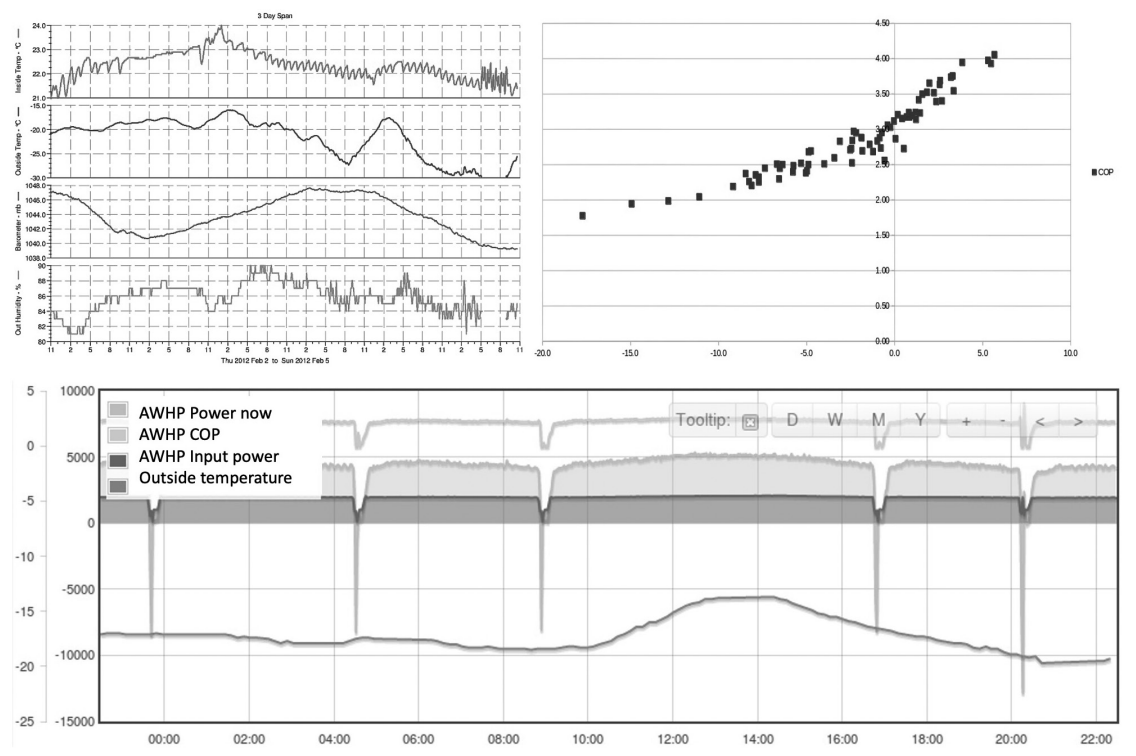

Figure 4.3 Users share heat-pump monitoring data graphs via forums and their own internet sites.

behave in temperatures that differ from the European standard of $+7^{\circ} \mathrm{C}$. The results tend to show occasions and/or areas where one's installation could be improved, and the forums feature active discussions about theoretical matters in heat-pump functioning, as well as discussions on trials of changing values and working around sensors. These comparisons also typically involve hacked and redesigned devices, as many hacked systems feature extra sensors and measurement points and are run by enthusiasts - indeed, many lead users report having gotten a cheap "toy air-heat pump."

Further on, many improvement projects are documented meticulously, picture by picture, and there were also attempts to create lists of useful DIY parts, as well as step-by-step (including pictorial) instructions on how to order, install, and use specific tools that are useful for monitoring and DIY work, such as Mango and a PolluCom energy meter, that spread hacking skills within the user group. Using some of the same parts and DIY tools also spreads competence and promotes collaboration on further modifying and inventing, in effect increasing the user base and the (mini)market for DIY parts and assemblies. This spread of competences is critical for heat pumps that have a mix of digital, electric, cold gas, and plumbing parts, which makes few people experts in all aspects of the technology.

Users are part of an ecology of producers, resellers, importers, regulators, bodies giving professional training, etc., as well as being part of peer-to-peer 
networks such as those found in user forums. Many, if not most, people engage in peer-to-peer networks because of a lack of needed information elsewhere and gradually change their perception, occasionally moving from an apprenticeship position toward increasing mastery. In the words of an interviewee:

Well, when you first buy a device from the store you sort of expect that now you have it [all sorted out]. It is not exactly a natural response to open it up and start messing with its internals, losing the warranty, and spending a fair deal of time on it. But then you encounter problems and oddities, go to forums and see that others are not so shy about examining these devices and reporting fixes and improved energy yields. It becomes more natural to see the product as not so perfect, to learn about the topics more. After a while you find that you have implemented a few simple hacks and there was nothing to it. That would have been simply unfathomable upon first encountering these devices.

(User with a GSHP, an ASHP, and micro-wind power)

The aspects of the systems that users who deepen their competencies come to master (moving beyond their initial competences) include terms and notations (e.g., assembly diagrams that are different for all technical subsystems), background theories (e.g., coolant gas thermodynamics), skills (e.g., attaching sensors), means (e.g., programming languages), regulations (e.g., the licenses that are needed to work with each part of the technology), and cost-benefit issues (e.g., compromising reliability in some situations for increased yields in others). This knowledge determines what one can do with the technology, as well as what governs which sections of the forum one can participate competently. ${ }^{3}$ These learning trajectories fit well with the idea of learning as legitimate peripheral participation (Lave and Wenger, 1991). Its prevalence in S-RET, particularly in regard to heat pumps, owes much to the technology combining several distinct specialist domains (in case of heat pumps: coolant systems, electronics, software, and in many cases also plumbing). All of the interviewed user inventors had started with modifications for which they had background competence: coders built software, electronics engineers hacked sensors, and so on. None had the competence to bridge to a more overarching (re)design from the outset - this emerged gradually over the course of their engagement, often over the course of months or even years:

By and large, I read [those posts] for about a year, and then, when I started to understand a bit, I did a bit more intensive reading; you see, thermodynamics presents a bit of a tricky domain.

(User with a modified GSHP)

It is noteworthy that internet discussion forums were conducive to legitimate peripheral participation to a far greater extent than the open-source 
development projects analyzed by Freeman (2007), who took part in our internet ethnography. The open-source project "OpenOffice.org," which she followed ethnographically for seven years, featured the frequent turning down of volunteers who did not have the required programming skills from the outset (i.e., the project remained open only insofar as one was already competent or close to being competent at the activities in which the community is involved). The internet forum's DIY sections feature some of the same characteristics: some naïve questions do not receive responses or merely get a referral to a thread elsewhere in the forum. Yet the forum supports myriad other ways to participate other than self-designing, and they are also more open-ended as to what is being done as part of community membership, including using, sharing, comparing, planning, theorizing, and visioning acts. In contrast, most open-source participants are involved in testing and in making small additions to the software. Hence, while user forums are less organized and less coordinated than open-source projects in their design activities, the former also have important strengths in relation to attracting and deepening usership and the growth of lead-user characteristics. The user-run renewables forums differ markedly from a clear innovation community, as well as from a clear community of practice, as the forum participants have a wider range of orientations, expertise, and practices that connect them to the forum. The diversity may be best conceptualized as a boundary infrastructure that is partially shared by several intersecting social worlds (Bowker and Star, 1999).

The learning and inventiveness found in these forums tie them to being run by peers. Those of our interviewees who had been following forums in other countries stressed that English-speaking forums had, in their assessment, curbed user modifications and critical evaluation due to being hosted and moderated by suppliers. Our informants equally stressed the importance of some of the administering solutions being conducive to success. These include light but active moderation, strong segmentation of the manufacturer-specific parts of the forum, a DIY section, and a debate section "hot ring" to which moderators move controversial and contested content and hence only have to dismiss content that is truly libelous or out of the scope of the forum. This was seen as favorable to the outcome of multiple specific orientations coexisting within a forum.

\subsubsection{Peer assistance for inventive user projects}

The most in-depth learning in the forums pertained to DIY projects and inventive users. Interviews revealed that all inventive users had some presence in the internet communities. These interviews and analysis of the discussion threads indicate that the inventive users received various kinds of help from other users, most commonly from two to five people. The most common responses to questions or flagged projects expressed different forms of community memory and expertise, such as weather an idea was worth pursuing or, more commonly, that something similar had been tried before. Typical 


\section{Internet-based energy communities}

question-answer sequences related to posting an idea of a modification project can be illustrated with the following exchange (see Figure 4.4):

The discussion in Figure 4.4 gives the project initiator a fair opinion on whether his suggested hack can be done and has, in fact, already been tried, as well as some of the likely issues he may be faced with. Our interviewees regarded these kinds of peer responses to be highly valuable:

The greatest benefit is that it has experts that really know what they are talking about. And regarding these specific areas they have [expertise in], you could say, they have an expert's and experimenter's attitude. That is what is interesting and useful. You get information on many things that would be very hard to get from elsewhere.

(User inventor for ASHPs)

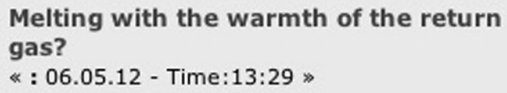

Is it possible to thaw the outdoors unit with the gas that returns from the indoors unit that still has some warmth in it? Put an extra bend onto the top of the outdoors unit bottom plate? Could one thus use after/waste heat of the gas for thaw instead of direct electricity?

Will such hach create problems with thawing, functioning of the device or efficiency or into the defrosting of the lamelles?

Red house and ...

Cellar +2 floors:

$4 \times$ Mitsubishi Electric MSZ-FD25VA+MUZ-FD25VABH

Re: Melting with the warmth of the

return gas?

« Reply \#1 : 06.05.12 - Time:13:59 »

Yes. Apart that its not gas at that point. Or if it is, something is wrong. The only problem you can encounter is that during the defrosting sequence and with reversed circulation the tube can freeze. Even though that doesnõt seem to create problem.

This one did not ice up the whole winter, apart a small bit in the rear end:

? https://picasaweb.google.com/...

Here is the number one version of AWHP thawing tube:

? https://picasaweb.google.com/...

Version two has that tube raised into the bottom plate, while I turned the water going inside and into the drain. Last winter is through without any need to interfere whatsoever.

Figure 4.4 The start of an exchange between members regarding a potential hack. 
Many user designs attracted further ideas, suggestions for solving problems, and direct iterations of design drawings posted on the forum. However, the users active in DIY threads formed a relatively small group of roughly 300 people that mostly had good technical competence in at least one or two technical areas related to heat pumps. Among the user inventors were also several researchers working within industry and academia, heat-pump professionals (such as those working as assemblers and resellers), and users with an entrepreneurial orientation in regard to furthering their business (e.g., in home automation). Gaining control of one's technical equipment, the joy of tinkering, training for their profession, and the possibility to stretch the limits of one's professional skills were all stressed as reasons to engage with self-building activities. Yet two issues featured in next to all interviews: the importance of forums for learning, and the importance of forums offering "a community of the likeminded," which motivated and justified one's tinkering. These observations parallel those made by Kuznetsov and Paulos (2010) and Grapher and Ibert (2014) on other internet communities.

Both an analysis of forum threads and interviews indicated that multiple modes of interaction, made possible through the forums, allowed users to turn their diversity into an asset rather than a hindrance. The forums allowed public postings and responses, as well as private postings and responses. The more advanced and more professionally oriented users used private messages as the predominant response medium regarding DIY projects and stressed the importance of anonymity as well as the possibility to then shift to a one-toone discussion out of forum-via e-mail or the phone. Their occupational reputation and commitments would have precluded them from playing around with heat pumps in the open. This range of interaction modes also played a major part in referrals of services, materials, and tools as many DIY projects and help given stretched, or even violated, warranties, insurance, and professional rules of conduct at some point in their development (cf. Torrance and Von Hippel, 2016).

Importantly, the relevant interactions were not limited to the small in-group of DIY participants. The larger community had been found indispensable for inventive users. In the words of one of our informants:

The heat-pump forum is a rather conservative site and [it is] not nearly as welcoming and inventive as people in the free-energy forum or pellet forum... [but], for example, the free-energy forum doesn't give much help for development work as there are too few folks in there.

(User inventor in ASHPs and wood pellets)

The attraction of new users and new fixtures is pivotal for gaining critical mass in the competences available. While most participants in user-run forums have no explicit intention to invent or facilitate other's inventive behavior, they inadvertently provide indirect support by doing what they do. Substantial numbers of discussions, instructions, manuals, photographs, 
76 Internet-based energy communities

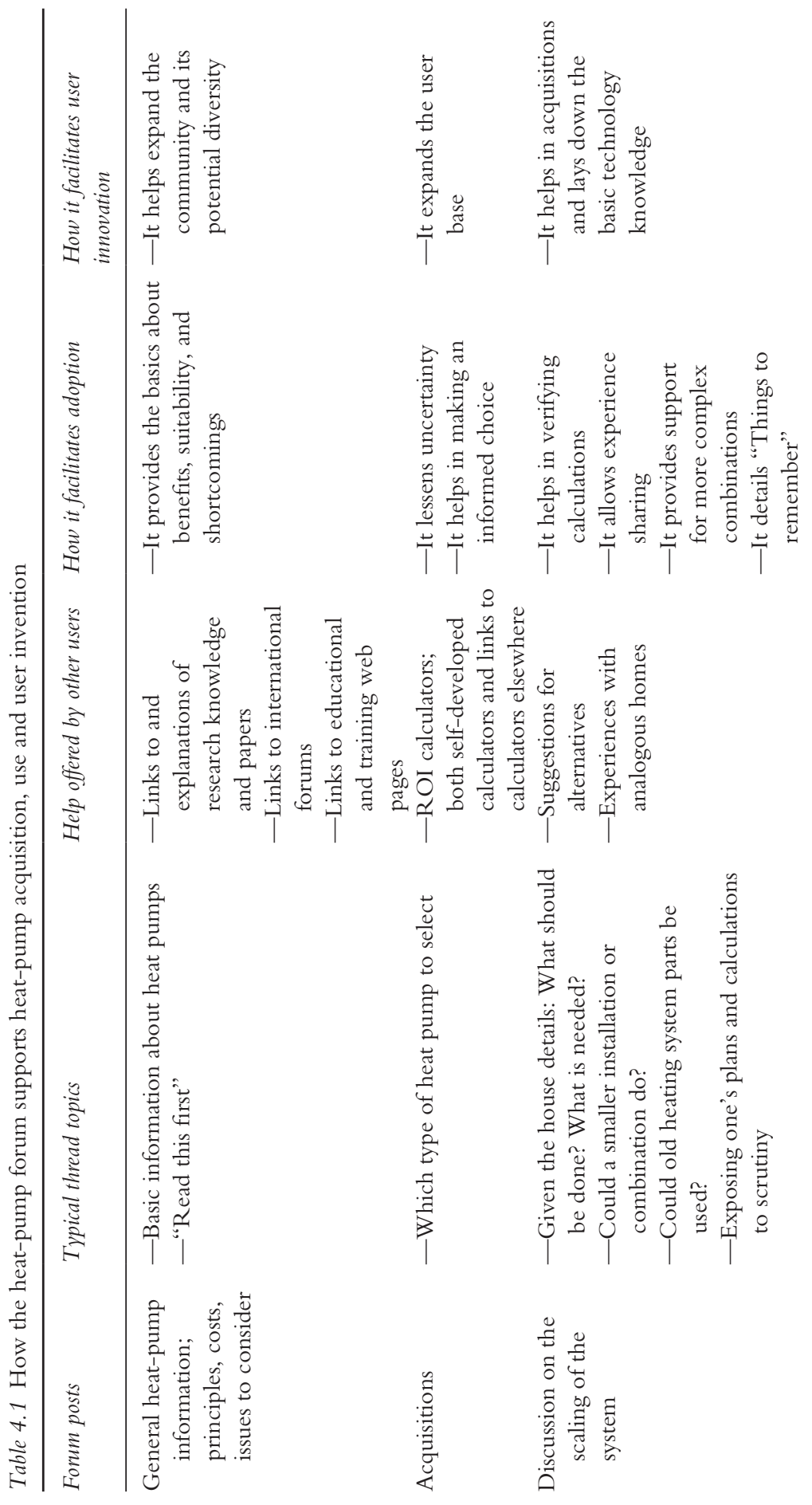




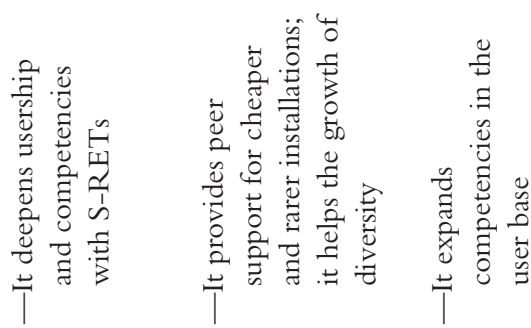

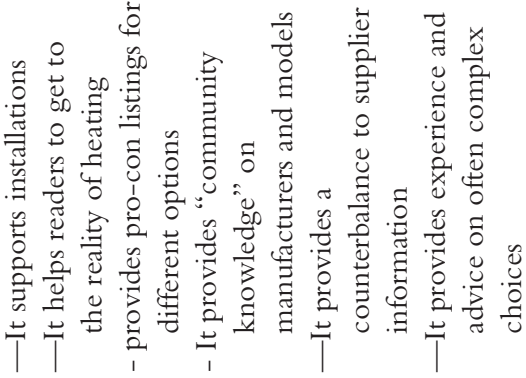
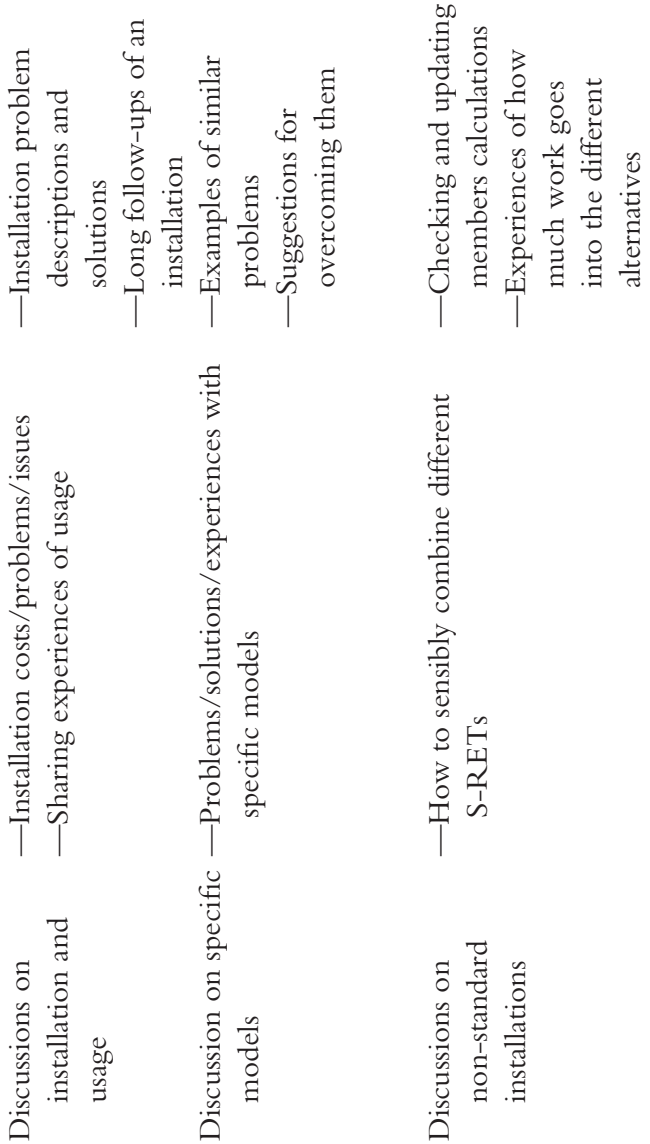
videos, links, calculators, etc., stored in the forum turn it into a shared toolbox and memory repository (Torrey et al., 2007, 2009). Further, the forum gives access to peers who contribute their insight into defining and solving posted problems, comparisons, voicing shortcomings, and taking part in the exchange of services and parts.

Non-standard installations are particularly relevant for the inventive user base, for example combinations of pellet, solar, wind, or heat-pump solutions. These projects create users that must grow quite knowledgeable about different aspects of their systems in order to make them work, and typically thus engage in peer-to-peer communications, regardless of if they personally go to the lengths of making hacks.

To summarize this section, internet forums have been important catalysts for learning how to use, adapt, and innovate for heat pumps in a cold-climate context. Peer-to-peer forums hold a special place with regard to the maintenance, proliferation, and further development of S-RETs. What becomes elaborated in the forums is not only what the technologies are and how they function, but also how they are being developed: what can be modified, how producers respond, how regulation responds, how other users embrace novelties, etc. These wider linkages are keys to proliferating the S-RETs to new types of installations and more complex installations, as well as to inventive solutions from their users.

\subsection{The systems change view: affecting the market and shaping technology}

\subsubsection{S-RET internet forums' growth patterns}

We now from an individual's perspective to the societal importance of S-RET related internet communities. The first internet forums for S-RETs were set up by citizen users after the first few thousand people had adopted the technology (in Finland between 2004 and 2008). The full statistics are only available at lampopumput.info and the discussion is anchored to that forum, which appears to be quite representative of the patterns elsewhere apart from its sheer size and growth rate that exceeds the other forums.

The growth of installed stock and forum reads has then been roughly linear, with reads growing at a faster pace ${ }^{4}$ (see Figure 4.5 , the lines in bold), so that the year 2006 had 19 reads per acquisition while by the year 2016 this had increased to 159 per acquisition (see Figure 4.5). As is common to such digital sites (Grabher and Ibert, 2014), readership has been much more numerous than instances of registering and posting questions or comments: for lampopumput.inf o, the cumulative reader count exceeded 2.5 million by 2017 , indicating that $1-3$ million visitors visited the forum in a language area of 6 million people. ${ }^{5}$

The numbers of new posts and new registered users rise until 2011 $(400,000$ installed systems or from less than $10 \%$ to $30 \%$ of estimated maximal diffusion) and then gradually decreased to roughly the early annual levels, 


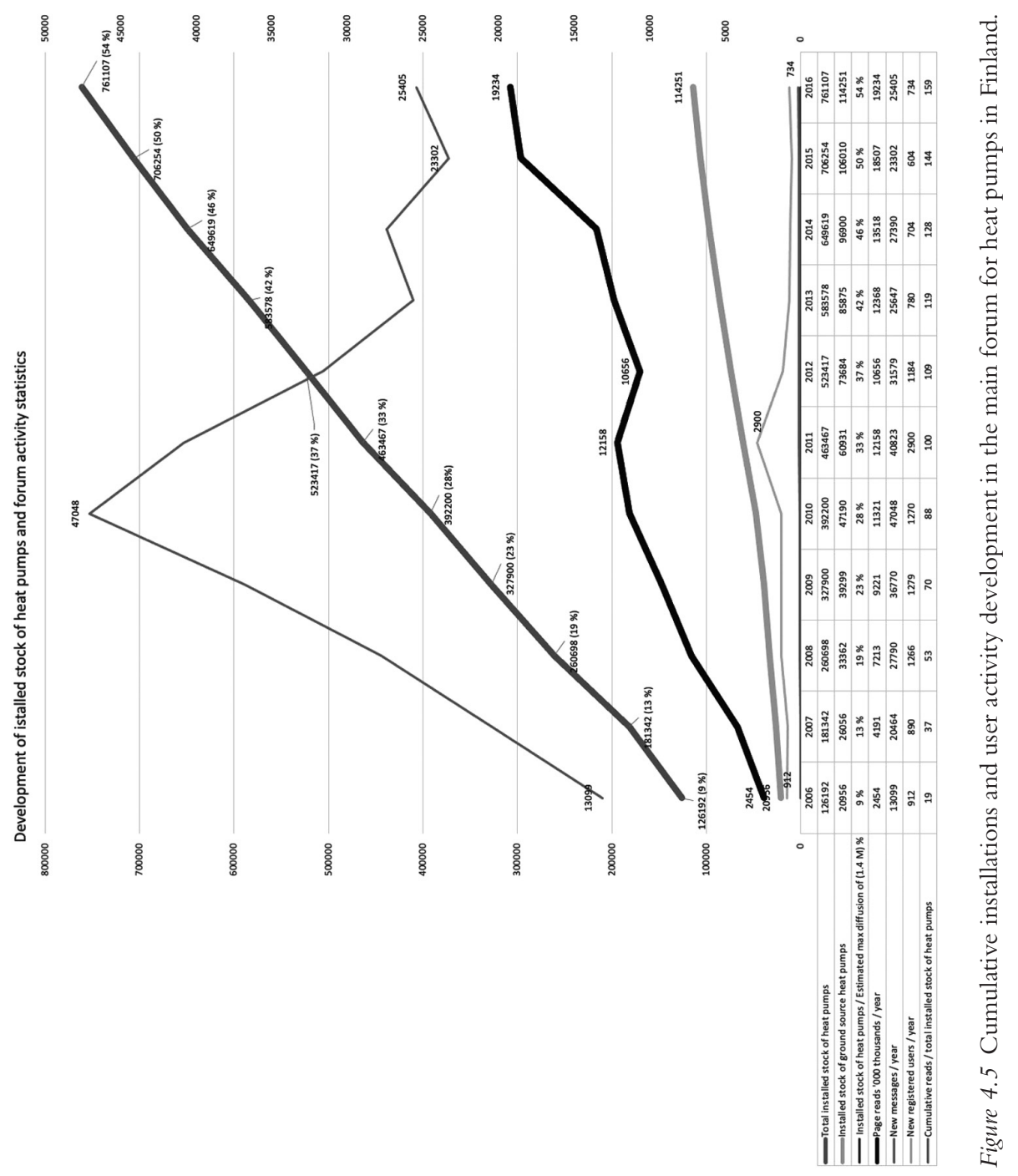


so that eventually to 5500 people, i.e., $0.1-0.5 \%$ of the readership register themselves, (see Figure 4.5). There are several simultaneous explanations for these changes. By this time, the accumulation of knowledge in the forum has become sufficient for an increasing number of new users so fewer people needed to register and post questions in order to satisfy their information needs in regard to acquiring and maintaining heat pumps. At the same time, the market developed so that merely acquainting oneself with the basics of scaling and selecting a heat pump became sufficient prior to purchase for most users. The later adopters may have also been less interested in the details of their purchases, as diffusion theory would suggest (Rogers, 2010). Yet, as the number of messages increased over the years, more pages became available for any given topic and users had more content they could read per query. The forums have also simultaneously served users contemplating the purchase of a heat pump and those who already owned a heat pump and needed to troubleshoot, improve it etc (see above section 4.2).

\subsubsection{Complementing market information: "independent peer knowledge" on technology and the market}

The information in the forum goes beyond a generic technology discussion aiming to educate consumers and has direct market-shaping characteristics. The forums make the available options easily accessible in terms of the choice of manufacturer, type, and model, and issues such as costs, installation, and maintenance. Each make and model has a specific section on the forum wherein users relate their experiences and suggestions, and the same goes for installation and maintenance. The forums commonly relativize manufacturers' brochures and adverts, and countenance exaggerated claims, typically stressing reliable brands with aftercare and warranties.

To evaluate the value of such complementary information in the market formation for new technology, it is instructive to compare the situation before and after the forums gained momentum in 2007 and after they had done so in 2012. In 2007 a joint-purchase project in the small municipality of Kangasala needed to deploy nine months of part-time work and a small EU project in order to determine the suitability and cost structures of the roughly 70 heatpump makes and models that were available then (Heiskanen et al., 2011; Martiskainen, 2014). After the forums had accumulated tens of thousands of posts, a similar search could be made in a matter of hours or at maximum, in a few days, and complemented with queries for references from people as to how they had done actual implementations. This is at once a massive gain in consumers' capacity to make an informed decision regarding a purchase and points to the complexity of making an informed decision on new technology of medium complexity such as S-RET systems.

Further on, as discussed in Section 4.2, forum benefits are not limited to making purchases and providing guidance on installation and use. These peer 
commentaries are sometimes paired with resellers seeking to assert their views as well, and the above introduced hot ring section for controversial topics and debates have kept the forum reliable without overblown censorship. When the forum scope and popularity among current and prospective consumers rose, it began to act as a community memory of both successes and failures. Together with the actions of the national industry association, the citizen forums have helped to keep the rapidly evolving market in check and to maintain its reputation among the wider public.

\subsubsection{Providing evidence of realized value and counter- evidence against claims of poor performance}

The performance of novel technologies tends to be subject to uncertainty, facing public counter claims made by incumbent actors as well as suffering from outof-date representations of yield and payback times in the face of rapidly developing product characteristics (Heiskanen et al., 2017a; Murto et al., 2019a, 2019b, 2020). Information on realized and comparable settings provided by trustworthy peers can be indispensable for addressing all these market(ing) problems, particularly if the market is in the consumer domain and not served by dedicated industry analysts (Pollock and Hyysalo, 2014; Pollock and Williams, 2016).

The common internet forum practice of displaying real-time performance curves with location and site information (see above) and the provision of very detailed calculators for estimating the likely efficiency in real conditions provide references for other adopters. As most forum participants provide their location, house specifics, and equipment configuration in their signature field, their monitoring activities (spurred by peer help in setting up monitoring) aggregated a repository of real-life measurements of heat pumps across different settings and outdoor and indoor temperatures. This, in turn, has allowed surveys and other comparisons to be made through the forums and made available therein.

The primary purpose of these activities has been to go beyond the manufacturer-provided information that has been tested in the standard test conditions for which the equipment is optimized (as all devices are) and to spot inaccuracies and errors in manufacturers' data. The secondary effect has been the capacity to also countenance the claims by other parties, such as research institutes, whose studies are actively and critically discussed in a specific section of the forum.

\subsubsection{Contributing to demand articulation and technical improvement}

The citizen users and their discussions in internet forums further contributed to direct and indirect demand articulation for the further development of heat pumps for cold-climate markets. In the beginning of diffusion, some users advocated cheap heat pumps over the early, expensive cold-temperature models. A decade later, the colder temperature models had become the norm and 
most advanced ASHPs retained a positive yield at $-25^{\circ} \mathrm{C}$. Large forum sections and user innovations devoted to discussions on winter performance had signaled to the importers and manufacturers that ASHP use in cold temperature called for improvement, and with the growth of the cold-temperature market size, this sent a signal to manufacturers to improve their cold-temperature models.

Another important development in demand articulation has been that citizen users began operating heat pumps along with other heating technologies. Whereas vendors and energy experts initially assumed that people would replace whatever heating they had with some S-RET, it became common to purchase an ASHP in order to complement existing heating systems that were based on oil or solid wood, as well as to complement an AWSHP and GSHP with solar thermal collectors. The resulting "hybrid heating systems" utilize the best yield time of each heating source relative to outside temperature and sunlight, which often requires working out the details and good switching points in between. Peer-to-peer information played a role in the market formation of such systems as the first vendors to sell a "technology independent assessment and renewable solutions" emerged in 2012 in Finland, over a decade after hybrid solutions started to become common. Hybrid S-RET systems also began to emerge stepwise in a pathway towards increasingly sustainable heating forms (Juntunen, 2014b).

\subsubsection{Peer-to-peer internet communities within the ecology of user-side intermediaries}

Consumers were not left to their own devices regarding S-RET. There were multiple other actors that mediated S-RET systems and their emerging market, forming what Stewart and Hyysalo (2008) characterized as an "ecology of intermediation." From the user perspective, however, these potential intermediary actors remained uninterested in many aspects of the heat pumps that were important for citizen users (research and academic institutions in particular), provided partial and self-interested assessment (resellers and installers in particular), or were difficult to reach and potentially offered too generic information for actual purchasing decisions (actors such as local energy advisors and a national energy efficiency institution). Some intermediaries, such as the technical press and mass media, provided basic information, price comparisons, and tests but only rather sporadically for a continuously evolving market and without evaluations of realized performance or delivery by different vendors. In Table 4.2 we detail what different actors in this ecology of intermediation mediated and what they did not mediate to citizens regarding heat pumps in Finland. The overarching finding is that each intermediary actor takes on activities that are sensible for themselves and thus only mediates, for instance, academic or within-industry knowledge, not what would be functionally optimal for the emerging niche or citizen users. The result is that the market institutions and intermediary actors are not likely to coalesce into an optimum 


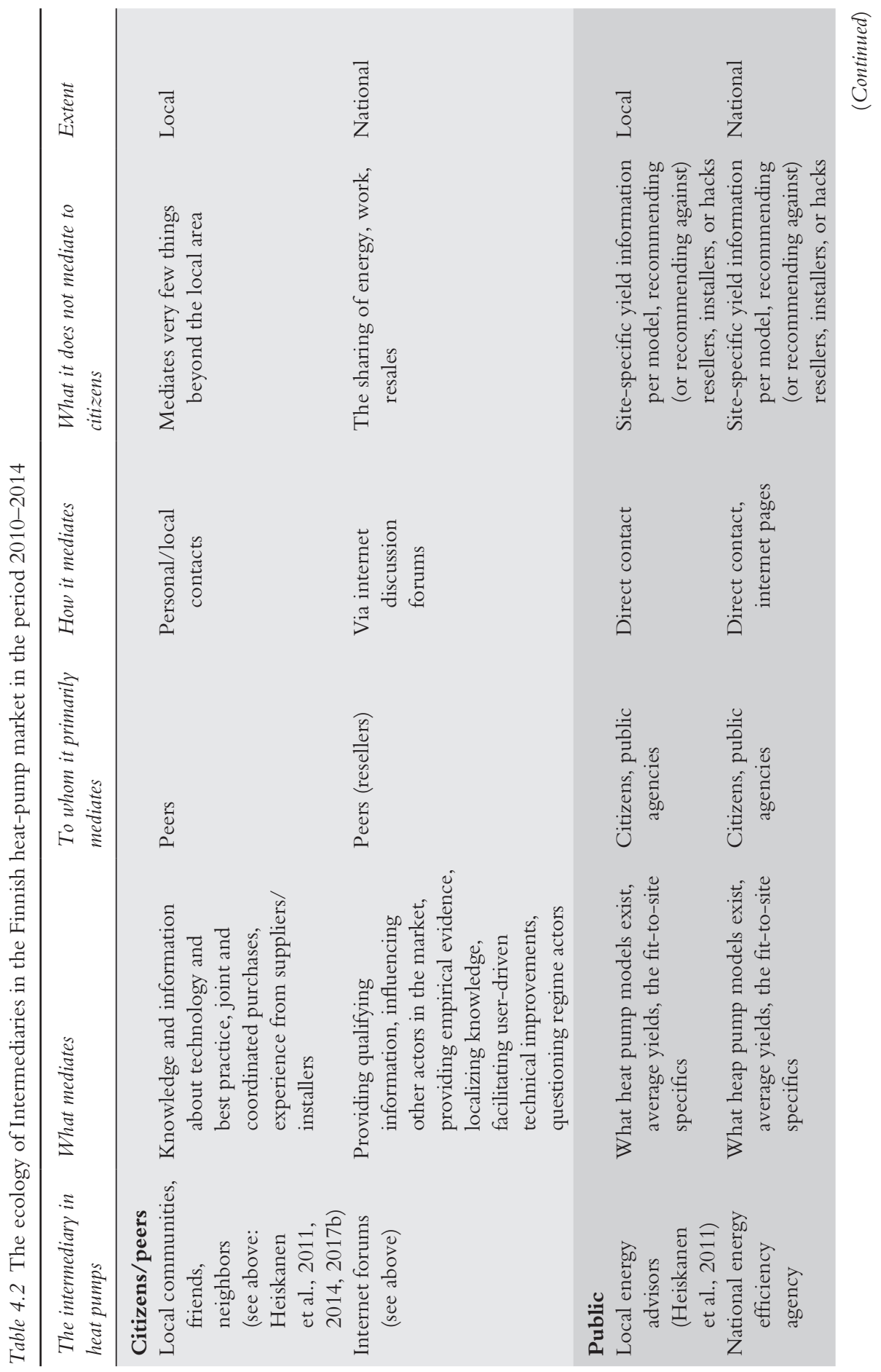




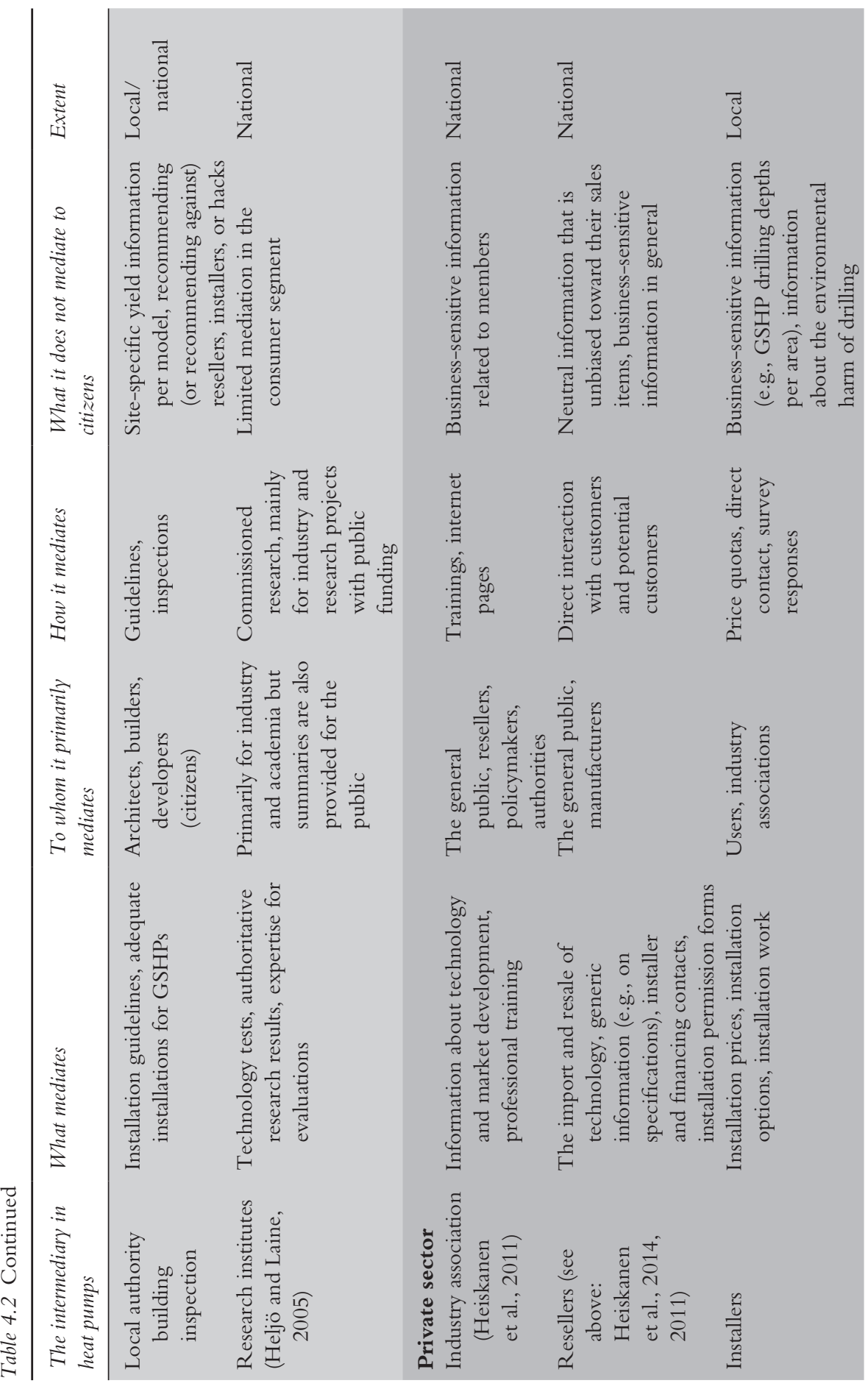




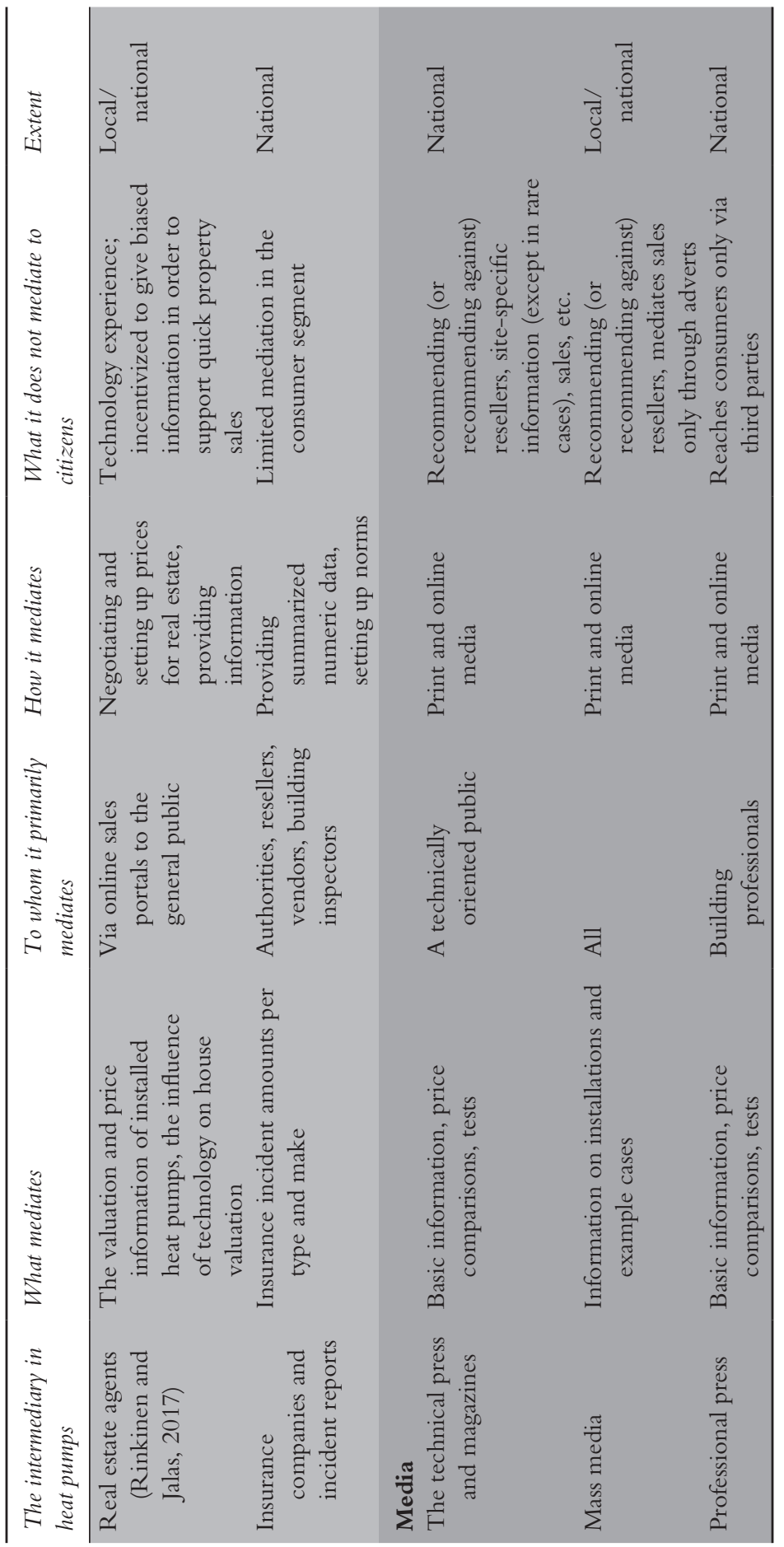


ecology regarding how citizen users could make informed acquisitions of novel technologies in the early phases of proliferation.

To summarize this section, from the systems change perspective internet communities can play an important role as user-side innovation intermediaries that ease adoption and the use of technology for the increasing number of new users, as well as being able to shape both market and technology characteristics. Whether this happens on a large scale depends on the ecology of intermediation at hand-if other actors have covered all the relevant information and support needs, there may not be a need for users to organize giving peer help or shaping market or technology characteristics. In such cases, users will likely be limited to giving only local and more limited user intermediation, as they do in virtually all other technology types.

\subsection{The comparative perspective on different energy communities}

\subsubsection{Fostering an appreciatively critical discourse on technology}

The previous sections have shown that new internet communities deserve close examination. In comparative perspective there are a few further characteristics that merit attention. The first of these characteristics is that these accelerationphase internet peer discussions feature relatively little critical, alternative, or pro-environmental discourse. Discussions revolve around and espouse the most seemingly neutral grounds of economic gain and technological optimization or improvement. When we further interviewed active forum discussants, those with explicit environmental motivations regarded that displaying them in the forums would merely lead to unhelpful debate. This runs in contrast to many community energy settings where the critical discourse on alternative technology has been found a key characteristic (Smith et al., 2016a, 2016b). Indeed, although the internet forum discussions are about clean tech and renewable energy, topics such as the reduction of carbon emissions are almost non-existent among the 300,000 messages of the main heat-pump forum and similarly so in pellet and solar forums. When such topics are mentioned, emissions are considered a problem on a higher level or part of political decision-making. In cases where technology is concerned, the emissions discussion is focused on other domains and technologies, such as transportation and cars, instead of on housing and heating. Open climate change skepticism can even be recognized in some exchanges.

Whilst this may appear odd at first there are important social dynamics at play. On the one hand, technical internet forums are a more widespread genre than the forums related to renewable energy - similar forums exist for bicycles, loudspeakers, various software products, and so on-and the implicit and occasionally enforced code of discussion in such settings does not involve issues that could be regarded as ideological or political. On the other hand, the appeal to technicalities and economics does important work in legitimizing and normalizing the novel technology and discussions about it. As many scholars of 
technology have remarked, a distinct characteristic of technology is its ability to mask political choices behind seemingly neutral, normal, and unstoppable progress (McKenzie and Wajcman, 1999). One could even argue that continuing to foster a widely critical alternative discourse on the technological options that are to be widely diffused is to strip them of the prime source of power that they may have as technologies within the discursive and political space of modern industrial societies. The mainstreaming and scaling up also run contrary to criticality among citizen groups, not just between the citizens and mainstream policy actors (Smith et al., 2016a, 2016b). The Finnish case of heat pumps indicates that such capping of critical discourse can be effective. Whereas heat pumps were disregarded by the experts regarding their suitability to the Finnish conditions throughout the 1980s and 1990s (Heiskanen et al., 2014, 2017a), by 2016 the aura of novelty had vanished. By then, heat pumps were viewed by the public and experts alike as the normal and rational choice for a heating system, and they were installed in the majority of Finnish detached houses. Throughout this time, the internet forum discussions opened and kept open the "black box" of technology in a critical enough manner for it to become appropriated, adjusted, and improved for the specific national context, but this was done appreciatively enough to protect the technology from the wholesale dismissals.

\subsection{2 (Local) community energy versus distributed and dispersed energy communities}

The second important aspect of the internet communities is how they contrast to traditional energy communities. Community energy activities have been commonly defined through their local participation: energy produced "by" and "for" stakeholders. In this they have an open, participatory, and collective character, even as particular projects vary in regard to just how open and participatory or local they ultimately are (Walker and Devine-Wright, 2008). In the community energy context, the community is often defined as a local unit that operates inside a limited geographical area. The community typically features a shared ownership and financing structure as well as shared decision-making rules. Often, the maintenance and further development of the S-RET is handled by the community, which contributes to the upkeep and deepening of energy competences among the community members. As noted, the community energy initiatives can further foster alternative critical discourse on technological options, which can present an important alternative to mainstream views and occasionally lead to the convergence of elements of community energy becoming adopted in mainstream energy policy (Smith et al., 2016b).

However, locality-bound community energy no longer appears to be the only important community form related to energy users. Distributed energy communities exist through shared energy production outputs over a wide area network, beyond the limits of a specific locale. Currently, these communities have emerged for medium- and large-scale renewable production units 
(Juntunen \& Hyysalo, 2015). Lumituuli, a wind energy company in Finland, and Solar Energy Cooperative Green Point Batensteinbad Woerden in the Netherlands, are examples of cooperatively-owned green electricity plants, owned by household investors who share the outputs of generation. Wind and solar collectives in these examples are characterized by distributed ownership, and they require only the minimal direct involvement of local people and of participants more generally beyond a small executive group engaged in the endeavor. Here, the outcome is not locally focused and the unit generates energy for wide distribution rather than for use in the locality (Cf. Walker and Devine-Wright, 2008). The recent development into smart-grids is opening the grid in new ways for peer-to-peer networking concepts and virtual power plants that can be used with small-scale production units and with renewable micro-generation technologies. The exact forms of these distributed ownership communities are multiplying as peer-to-peer networking concepts have brought new models of sharing to community energy systems (Juntunen and Hyysalo, 2015; Steinheimer et al., 2012).

The internet forums portray a digitally mediated community form in which geographically dispersed users share an interest in the same class of technology and in a digitally mediated infrastructure without committing to shared finance or produce. As described above, the locality-independent reach allows internet communities to achieve much wider networks and higher coordination effects across the user base (Grabher and Ibert, 2014; von Hippel, 2016), helping them to add momentum to accelerating the sociotechnical pathway (Heiskanen et al., 2011; Karnøe and Garud, 2012). As noted, in these settings the household users own their S-RET equipment and utilize self-generated electricity for their own consumption but are actively linked to peers who run similar technologies and thus face similar questions in acquainting themselves with the technological options, scaling the system(s), choosing from among the available brands, and combining different S-RET forms and implementing, adapting, and improving their systems. In terms of the three generations of findings on users in sociotechnical change outlined in the introduction, the community energy discourse appears rooted in the 1980s-1990s understanding of what and how citizens shape technology for themselves and others, and in so doing neglect the import of digital communities.

Table 4.3 demonstrates the key differences between the three energy community types: local community energy, distributed output sharing, and dispersed knowledge-sharing communities (Hyysalo \& Juntunen, 2018). Whereas in traditional community energy all aspects are dealt with locally, in distributed wind energy the project is owned and governed together and outputs are shared within the group or sold to other users over the grid. In dispersed knowledge-sharing communities, ownership and control over production are in the hands of each household. However, in terms of knowledge-sharing and learning processes, the household can enjoy the benefits of and contribute to a wide energy community wherein members share common interests on a much wider scale than in local and distributed forms. 
Table 4.3 Case examples of community energy: a local community energy project and a dispersed structure community

\begin{tabular}{|c|c|c|c|}
\hline & $\begin{array}{l}\text { A locality-centered } \\
\text { community energy project } \\
\text { (e.g., a wind project, a solar } \\
\text { project) }\end{array}$ & $\begin{array}{l}\text { A distributed energy } \\
\text { community, created } \\
\text { through output } \\
\text { sharing }\end{array}$ & $\begin{array}{l}\text { A dispersed energy } \\
\text { community through } \\
\text { knowledge sharing }\end{array}$ \\
\hline $\begin{array}{l}\text { Scale of the } \\
\text { production } \\
\text { unit }\end{array}$ & Small or medium scale & $\begin{array}{l}\text { Medium (or large) } \\
\text { scale }\end{array}$ & $\begin{array}{l}\text { Decentralized small } \\
\text { scale }\end{array}$ \\
\hline $\begin{array}{l}\text { Ownership } \\
\text { of the } \\
\text { production } \\
\text { unit }\end{array}$ & Community owned & $\begin{array}{c}\text { Community } \\
\text { owned }\end{array}$ & $\begin{array}{l}\text { Owned by } \\
\text { households }\end{array}$ \\
\hline Daily operation & $\begin{array}{l}\text { By an active group inside } \\
\text { the community (or } \\
\text { outsourced) }\end{array}$ & $\begin{array}{l}\text { By an active } \\
\text { group inside } \\
\text { the community } \\
\text { (or outsourced) }\end{array}$ & $\begin{array}{l}\text { By user, user } \\
\text { responsibility }\end{array}$ \\
\hline $\begin{array}{l}\text { Knowledge } \\
\text { sharing and } \\
\text { community } \\
\text { learning }\end{array}$ & $\begin{array}{l}\text { Social learning when } \\
\text { working together } \\
\text { locally for a common } \\
\text { goal }\end{array}$ & $\begin{array}{l}\text { Social learning } \\
\text { among those in } \\
\text { the community } \\
\text { who engage } \\
\text { beyond mere } \\
\text { output sharing }\end{array}$ & $\begin{array}{l}\text { Individual operational } \\
\text { work supported } \\
\text { by the online } \\
\text { community; } \\
\text { characterized by } \\
\text { common interest }\end{array}$ \\
\hline $\begin{array}{l}\text { The scale of the } \\
\text { community } \\
\text { knowledge } \\
\text { pool }\end{array}$ & $\begin{array}{l}\text { The participants in the } \\
\text { locally owned and run } \\
\text { community energy } \\
\text { project and their } \\
\text { personal networks }\end{array}$ & $\begin{array}{l}\text { The engaged } \\
\text { participants } \\
\text { in the energy } \\
\text { project and } \\
\text { their personal } \\
\text { networks }\end{array}$ & $\begin{array}{l}\text { Thousands of users } \\
\text { with similar } \\
\text { equipment and } \\
\text { a broad range of } \\
\text { competences }\end{array}$ \\
\hline $\begin{array}{l}\text { Governance } \\
\text { characteristics }\end{array}$ & $\begin{array}{l}\text { Organized; requires a } \\
\text { governance structure, } \\
\text { community control }\end{array}$ & $\begin{array}{l}\text { Organized; } \\
\text { requires } \\
\text { governance } \\
\text { structure, } \\
\text { community } \\
\text { control }\end{array}$ & $\begin{array}{l}\text { Household control } \\
\text { and autonomy }\end{array}$ \\
\hline $\begin{array}{l}\text { The distribution } \\
\text { of energy } \\
\text { production }\end{array}$ & $\begin{array}{l}\text { Microgrid or grid } \\
\text { connected; primarily } \\
\text { for a group }\end{array}$ & $\begin{array}{l}\text { Microgrid or grid } \\
\text { connected; } \\
\text { primarily for a } \\
\text { group }\end{array}$ & $\begin{array}{l}\text { Primarily for own } \\
\text { use; mostly grid } \\
\text { connected }\end{array}$ \\
\hline
\end{tabular}

The differences can be further illustrated by adapting the well-known community energy mapping of Walker and Devine-Wright (2008; Creamer et al., 2019). Figure 4.6 illustrates how the traditional and new forms of community energy contrast with a centralized wind power utility. Community energy can be seen as producing energy by open participation (Type A in the 


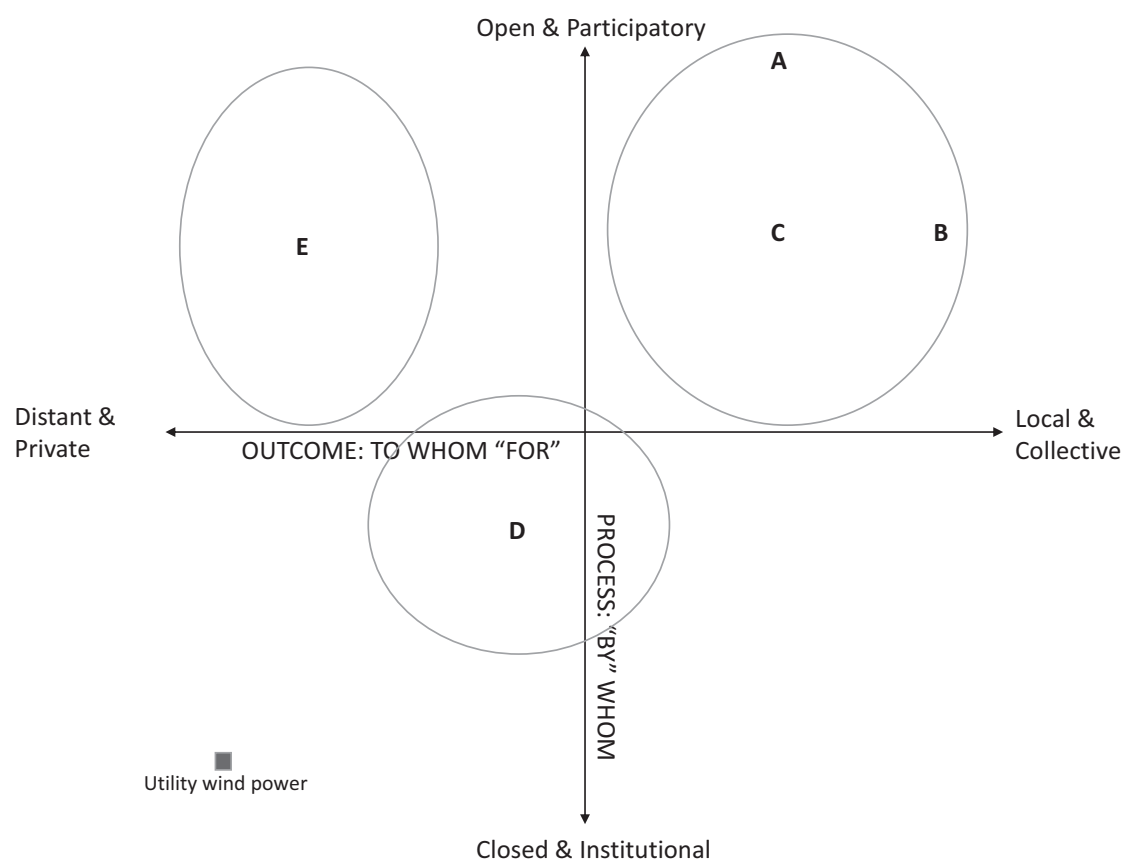

Figure 4.6 Mapping energy communities regarding the outcome and participation in a digitalized world.

upper right corner of Figure 4.6); others stress that community energy primarily generates energy for community benefit (Type B); whereas in practice there is greater variety between and within community energy projects (area covered by Type C). Despite these differences, all these conceptions assume that energy communities involve important citizen participation and cooperative output distribution, which must be local and open. This is not the case with the distributed energy community that features distributed output, such as a medium scale wind cooperatives (Type D), which would clearly map away from the local end and, in most empirical cases, also away from most intensive forms of participation, typically having only a limited number of active members running the cooperative whereas others are involved mainly through financing and output sharing. The dispersed energy communities, such as the internet discussion forums (Type E in Figure 4.6) map onto the graph towards being distant and private in terms of outputs, but towards being open and collaborative in terms of participation, action, learning, and cooperation.

The form of digitally dispersed knowledge-sharing forums appears to matter as well. Unlike in the S-RET communities hitherto studied, the globally 
dominant form of digital user community has had a company brand focus and sole control of the forums. The study of user-run online forums indicates that company-run online forums for a single brand-for example, the Ducati motorcycle community (ducati.kontain.com) or the Apple support community (discussions.apple.com) - provide only limited usefulness from a user perspective. Before acquisition, help is needed in order to compare different competing solutions from various vendors, not just from one provider. During the use period, products are embedded in practices of everyday life and use is typically linked to other products and, in active design engagements, it is by default that users tinker with more than one manufacturer offering.

As to the factors that may drive users toward local community energy or individual S-RET deployment, the differences are not decisive. Both provide returns on investment for owners, and give local control and power to make energy generation decisions. However, some factors favor householdspecific installations. Planning permissions are typically easier the smaller the production scale. In heat production, a smaller distance between generation and use results in lesser losses in energy transmission. Organizational and contractual complexity around individual installations is smaller than in medium-scale community energy, which means greater agility in the set-up process. The peer support and expertise available in internet communities has begun to compensate for the knowledge based and competency benefits the community energy previously held over individual installations. It is thus foreseeable that increasing virtual connectivity among citizen users may create new, dispersed community forms both in S-RET production and in linking producing users with those who have storage or a load (Juntunen and Hyysalo, 2015, 2018).

\subsection{Chapter conclusions}

S-RET internet forums enable and facilitate peer-to-peer exchanges and knowledge accumulation, which other actors in the emerging market do not, or even cannot, provide (Grabher and Ibert, 2014). Through internet communities, citizen users gain easy and quick access to technology and market information, and they gain a polycentric knowledge repository that helps balance the various self-interested assessments around new technology. This helps in scaling the system to one's heating needs, choosing from among technological options and product alternatives, and comparing different vendor offerings. The forums provide a learning environment on the S-RET and its deployment in real-life contexts, including comparing heating systems' ongoing operation and performance, troubleshooting and maintenance, and DIY projects and inventions.

The internet communities strive only if they can accumulate a sufficient mass of diverse participants, who then have the interest and competencies to 
address the varying issues their peers have regarding the new technology. In this capacity they are best conceptualized as forming a boundary infrastructure (Bowker and Star, 1999), which appears effective in fostering opportunities to learn through legitimate peripheral participation and deepen competences (Lave and Wenger, 1991) and also in facilitating their participants' learning, progressing from apprenticeship to mastery across domains of competence. In this view, many inventive users are not "born" but "grow" to have the capacities, and special needs/wants that drive them toward invention. Userrun internet forums are conducive to the deepening of usership and allow some of the other users to act as user-side intermediaries to aid this. An implication of this argument is that the study of user innovation would benefit from more careful treatment of the nature of communities and types of user participation. Equating user communities with innovation communities, or drawing a demarcation line between inventive and non-inventive users, masks important cross-overs and areas worth researching.

The gains consumers make through participation in the internet communities affect systems change at the aggregate level. For buyers the forums complement the information available on the market on technology and product options and add transparency and trustworthiness to the new technology through the possibility to evidence peer experiences and use similar cases as references to one's own acquisition process. The community provides a channel through which the consumer voice can become expressed regarding satisfaction with supplier offerings, as well as shortcomings and failures in sales, performance, installation, and maintenance. This complements industry association efforts to maintain the quality and trustworthiness of a growing technology field, as does the community tendency to qualify incumbent slants and calculations made by research institutes with real-life data.

The discussions, installation types, and modifications displayed by peers further contribute to demand articulation regarding further technology development. In sum, the internet communities can play a substantial role, both at the scale of the individual participant and at the aggregated scale of market and technology shaping.

In all these aspects, the locality-independent reach allows internet communities to achieve wider networks and higher coordination effects across the user base than local communities or distributed cooperatives could, and in so doing they add momentum to the accelerating sociotechnical pathway (Heiskanen et al., 2011; Karnøe and Garud, 2012). These communities also allow higher variety in participant motivations and participation goals, and require less member specialization than, for instance, open-source software development projects (von Krogh and von Hippel, 2003; Hyysalo et al., 2013b; cf. Grabher and Ibert, 2014). As a consequence, as important as the grassroots innovation movements and community energy initiatives are at the start-up phases of energy transitions (Hargreaves et al., 2013; Smith et al., 2016b), the wider proliferation of new technology benefits from different 
aggregation and exposure capacities, which the more diverse orientation internet communities can create. ${ }^{6}$

Regarding the literature on intermediaries in innovation and transitions (Howells, 2006; Stewart and Hyysalo, 2008; Kivimaa et al., 2019a, 2019b) the peer internet communities underscore the contingencies regarding who are the actors within an emerging industrial field that take on specific userside intermediary roles - the ecology of intermediation can take many forms and remains subject to change as the field evolves. Within such an evolving ecology, citizen users can play an important part in reconfiguring dispersed knowledge resources to locally relevant assemblages, brokering connections between peers and suppliers, and facilitating learning about new technology and relevant actors, as well as learning about being a user of a new technology. Importantly, in the world of internet-mediated connectivity, peers do not need to be physically co-located for many of these activities to become effective, unlike what was assumed by the early concepts on user-side innovation intermediaries, such as warm experts (Bakardjieva, 2005) and local experts (Stewart, 2003).

Finally, in terms of policy implications, technology-specific online forums feature several behaviors that Nye et al. (2010) hypothesized as leading to behavioral change. Inventive and actively monitoring users set an example to others by deepening engagement with energy technology, arguably leading to a higher consciousness of how much energy is consumed and how it has been produced. Easy modifications and add-ons appear to give greater ownership of and visibility to energy issues that tend to become "infrastructural" and not be considered.

With regard to facilitating internet communities themselves, it may be illusory to seek to create such discussion forums through policy measures, but allowing (often anonymous) discussions to prevail holds benefits, and some energy and climate policy actors, such as energy councilors, might offer their advice through these discussion forums, as has already happened in youth work after the realization that it is increasingly on the internet where people can nowadays be reached. Some forums may benefit from nominal support in order to cover running costs. Companies could also volunteer to further develop their best DIY ideas with a compensation scheme in place. Best arrangements in policy, co-design, and company involvement are, however, likely to vary with respect to the type of forum, technology, and user base in question. The forums examined here feature several facets relevant to those trying to set up and facilitate technology-related internet communities: segmentation of the community into separate sections to facilitate the co-existence of different user orientations, including a separate section for provocative and speculative exchanges; active but tolerant moderation, which primarily refers discussions to appropriate areas; and allowing some forms of private messaging and anonymous presence, which allows different professionals to engage in projects and speculation without reputation loss. 
From a BOAP methodology perspective, the analyses of S-RET internet communities display an expansion pattern, in which the original research interest in user innovation was gradually superseded by an interest in seeking to understand how the inventive activities were embedded in internet-based peer communities. Consequently, the peer learning, competence building and peer-intermediation activities were studied more closely and it became apparent that these communities ought to be studied for their own right to understand what all happens there and to what effects. This entailed a shift from innovation studies framing toward S\&TS that was better equipped for studying internet communities and characterizing them. A further expansion in the focus of investigation ensued once we moved from studying individual users and what the internet communities may offer them to wider ecologies of intermediation and the role that the user communities play in the wider sociotechnical change. This meant also bridging the study to a new set of reference literature on energy communities and energy transition to make sense of how the nature and kind of transition relevant energy communities may have changed. The concluding chapter will address in more depth how these focal shifts in research program relate to tracing the series of configurational movements (Hyysalo et al., 2019a) that comprise the overall import of users in sociotechnical change, but to do so we first need to zoom out more and examine the range of user activities in Finnish energy transition, which we do in the next chapter.

\section{Notes}

1 This chapter integrates and expands research that has previously appeared in Hyysalo, S., Juntunen, J., and Freeman, S. (2013b). Internet Forums and the Rise of the Inventive Energy User. Science and Technology Studies, 26 (1), 25-51, in Hyysalo, S. Juntunen, and J.K, Martiskainen, M (2018) Energy Internet forums as acceleration phase transition intermediaries. Research Policy 47 (5) 872-885. Short sections have also previously appeared in Hyysalo, S. and Juntunen, J.K (2018) User Innovation and peer assistance in small scale renewable energy technologies. In Davidsson \& Gross (eds) Energy in Society Reader. Oxford University Press.

2 See the full discussion at http://lampopumput.info/foorumi/index.php?topic=1375.0.

3 It is worth noting that there is hardly any single schooling that would prepare someone to have the encompassing expertise in all the system areas involved here. The professionals, too, have learned many of their skills out in the field.

4 One should add $20-30 \%$ more reads in the GSHP forum in order to get the full picture regarding heat pumps.

5 The cumulative reader count is measured from unique IP addresses. The IP addresses from an organization can show as only single address but also people can have several IP addresses from which they access the site. The rule of thumb for counting real visitors from IP addresses varies; typically, there are more visitors than IP addresses, but given the decade-long timespan here, we use a conservative and broad estimate of 1-3 million visitors.

6 Similar arguments have been made between locality-based user innovator communities and Internet-based open-source communities (Baldwin and von Hippel, 2011; von Hippel, 2017). Regarding energy transitions, we can also observe some carry over between user intermediation from an already widely diffused niche technology to another, such as when peer advice in heat pump Internet forums proliferates into other, 
less-diffused renewables for the purpose of creating hybrid heating systems for yet higher eco-efficiency in a specific national context (Juntunen, 2014a, 2014b; Hyysalo et al., 2013b, 2017). These domestication and diffusion pathways are a research area that merits further attention. We can also conjecture that digital communities truly prosper if a critical mass of discussants can be found and are thus not likely to be as effective as the predominant form of community as local communities are in the early explorative stages of the development of alternative new technologies. 


\title{
5 Zooming out \\ User activities and the series of configurational movements in energy transition
}

\author{
Sampsa Hyysalo and Jouni Juntunen
}

\subsection{Introduction: transitions as a potential framing to integrate different user contributions to sociotechnical change}

The idea of sociotechnical transitions may hold the potential for integrating and examining the interrelations between different user contributions in long-term sociotechnical change. Transitions research examines the long-term change in sociotechnical systems under the conditions in which consumption and production patterns are ingrained in existing structures (Geels and Schot, 2007; Köhler et al., 2019). Under such conditions, the often radical changes needed to increase environmental sustainability face the inertia of existing sociotechnical regimes. Regimes feature shared rules and the intertwinement of the technology base, scientific research, logistics, raw materials access, investments, regulation, and consumption patterns, which have formed strong path dependencies and efficiencies through decades of sunken investment and learning effects (Rip and Kemp, 1998; Geels and Schot, 2007). In transitions research, systems change is seen to be possible through the destabilization of dominant regimes by landscape pressures and the gradual technical, economic, and sociotechnical maturation of alternative solutions in niches, which are partially protected from the full selection pressure of the market (Geels, 2002; Geels and Schot, 2007; de Haan and Rotmans, 2018).

Transitions are seen to follow a progression of stylized phases, beginning from the pre-development and exploration phase where small-scale experiments for new alternatives take place, but no visible change happens in the regime (Hoogma, 2002; Geels and Schot, 2007; Safarzynska et al., 2012). The take-off phase follows, wherein the alternative technology develops into a niche, with more developed technical characteristics and market availability, and gradual agenda building around the niche (Safarzynska et al., 2012; Geels and Schot, 2007). The next phase is the acceleration and embedding phase, during which niches expand and become mainstream markets that start to compete with the incumbent regime, which then begins to react to the niche (Kanger and Schot, 2016). The expansion of the adopter base is associated with structural changes in markets and institutions, and with the continued development

DOI: $10.4324 / 9781003133919$ 
of technological solutions, gradually improving their economies of scale (Safarzynska et al., 2012; Kanger et al., 2018). The final phase is stabilization, marked by the decreasing speed of sociotechnical change as a new dynamic equilibrium is reached, and it becomes easier and more routinized for adopters to make a choice in the new regime than in the old (Geels and Schot, 2007; Schot et al., 2016; Geels et al., 2016).

Ambiguity remains regarding the markers of transition phases. Geels and Schot (2007) tie the acceleration phase both to innovation diffusion theory (Rogers, 2010) and to the point when the diffusion curve becomes self-sustaining, implicitly cast somewhere between $5 \%$ and $20 \%$ of the total cumulative adoption. Some authors denounce the split between pre-development and take-off (Schot et al., 2016; Kanger and Schot, 2016), while, for example, Meelen et al. (2019) claim to study the acceleration phase at the point of less than $2 \%$ e-car diffusion, implying that if other systemic properties were sufficiently in place, acceleration could be seen to take place much earlier despite low cumulative adoption. Reflecting on this high variance, the transition phasing is perhaps best seen as a useful means for the indicative comparisons of the stages of system change in different contexts, rather than providing strict operationalization thresholds.

The benefits of transition framing, and the reason why it was used in the present study, are that it connects the technology proliferation to the advancing technology and market characteristics, and thus holds the potential for clarifying the interconnections between the various activities that adopters perform in the course of sociotechnical change (Heiskanen et al., 2014; Schot et al., 2016; Kanger et al., 2018). As noted in Chapter 1, the capacity to connect different aspects of user activities is important as several fields have contributed to understanding different aspects of how user activities may contribute to sociotechnical change (McLaughlin et al., 1999; Hyysalo et al., 2016a; Kohtala et al., 2020). The economics of innovation and innovation systems have long recognized the importance of producer-user interactions (see, e.g., Rosenberg, 1982; Lundvall, 1988), and included users among the actor groups that affect innovation systems via shaping the selection environment, market creation, and direction of search activities (see, e.g., Freeman, 1979; Weber and Rohracher, 2012; Bergek et al., 2015). Yet to understand in more detail what and how users contribute to sociotechnical change, several related disciplines and strands of innovation studies provide greater detail and clarity.

The proliferation of new technology is not reducible to the communication of unchanging goods in undifferentiated social systems (Rogers, 2010) but involves changes in the composition of goods, markets, and the institutional environment (Mackenzie and Wajkman, 1999; Sørensen, 1996; Williams et al., 2005). The adoption process is qualitatively different for the adopter segments that follow the early, typically technologically savvy, adopters: the requisite signaling of availability, the social legitimacy of adoption, payback characteristics, observability, the understandability of the new solution, and the ease of adoption all need to be at a higher level in order for the 
later adopters to adopt - the technology and market may have to change substantially in order to spread to new segments (Rogers, 2010; Moore, 2001; Cockburn and Ormrod, 1993; Williams et al. 2005). Transitions literature acknowledges these qualitative shifts in adoption through its emphasis of institutional, market, and technology change in the "societal embedding" associated with acceleration phase (see, e.g., Hoogma et al., 2002; Kanger et al., 2018; Meelen et al., 2019), but this and the user activities associated with it merit closer attention.

Further, as discussed in Chapters 1 and 4, taking goods into use often involves not only adoption but also adaptations, such as resignifying, repurposing, adding to, modifying, redesigning, and intertwining the goods with other artifacts, physical contexts, and everyday practices (de Sanctis and Poole, 1994; Juntunen, 2014a; McLaughlin, 1999; Silverstone and Hirsch, 1992; Kohtala et al. 2020). Adaptation can increase the adoption of innovation by making it possible to adjust it to the cognitive, social, and material needs of the adopter (Agarwal, 1983; Fleck, 1993a, 1993b; Rogers, 2010). Indeed, the applicability of off-the-shelf small-scale renewables to a given adopter site varies owing to region-specific variations in housing, climate, and regulation, as well as to the building location, housing type, and homeowners' everyday practices (Judson et al., 2015; Juntunen, 2014a; Nyborg, 2015). Adaptations are often paired with various forms of championing complex projects, new installations, and information gathering (Klerx and Aarts, 2013; Martiskainen and Kivimaa, 2018).

As discussed in Chapter 3, some users may become innovators themselves. Research on the early phases of an energy transition has underscored the importance of citizen groups working as activists and innovators, initiating niche development in wind turbines, solar collectors, low energy housing, and alternative building types (Ornezeder and Rohracher, 2006, 2013; Truffer, 2003; Seyfang, 2010; Nielsen, 2016). Some of these civilsociety initiatives have fueled mainstreamed development activities while others have remained local, critical endeavors for alternative ways of future life (Hargreaves et al., 2013; Smith et al., 2014, 2016b). As we learned in Chapter 3 , innovation by users has also been found in the acceleration phase of transition in line with innovation being found among the general consumer population (von Hippel, 2016).

Throughout Chapters 3 and 4 we saw how adoption, adaptation, and innovation processes were amplified by peer intermediation and peer communities. Again, transitions research has mostly concentrated on community groups and movements that are united by an ideological commitment to alternative technologies and are typically geographically local (e.g., Smith et al., 2016b). But, as discussed, there are also increasingly important communities that are native to digital settings that connect the geographically separated peers of alternative technologies (Hyysalo et al., 2018; Meelen et al., 2019).

Transition research has further emphasized advocacy and political participation by citizens as key civil-society contributions to transitions, following 
earlier work on social movements and in science and technology studies (e.g., Smith, 2012; Jørgensen, 2012; Hess, 2005). This emphasis is, on the one hand, due to the fact that most energy- and mobility-related societal developments are intertwined in political decision-making, either in preserving the status quo or in deciding to favor alternatives that are typically initially more costly and uncertain, such as the potential of wind and solar energy up until the late 2000s (Rip and Kemp, 1998; Hoogma et al., 2002; Truffer, 2003; Smith, 2012). On the other hand, tying citizen participation to political processes is due to an association to the environmental movement, which has been common in the early years of the formation of many sustainable niches (see, e.g., Ornetzeder and Rohracher, 2013; Hess, 2005).

Research aiming to clarify actor roles in transitions have been common (e.g., Farla et al., 2012; Bergek et al., 2015; deHaan and Rotmans, 2018), but there has been less than a handful of studies that focus specifically on civil society and users in the course of transition and that are not limited to a temporally and spatially limited snapshot. Smith (2012) reviewed the literature on traditional civil-society-influencing mechanisms for energy transitions. He views grassroots innovation, citizen science, and green consumption as early nichesupporting processes that lead to the following: the community-led upscaling of innovation; consumer boycotts, protests, lobbying, counter-expertise, and standard creation as regime-destabilizing forces; awareness raising and social pressure as landscape-level pressuring processes; and community aspirations and the emergence of plural visions in civil society as processes falling between matured niches and the incumbent regime. Smith further illustrated how civil-society action could have substantive, procedural, and structural destabilizing effects. While Smith focused on civil-society activities, Schot et al. (2016) phrased their review in terms of users, potentially expanding the scope from the various forms of civic activism to cover all the affected people on the demand side of the transition. They propose a sequence where "userproducers" and "user-legitimators" create technological and symbolic variety during the start-up phase, "user-consumers" integrate the solutions into their everyday lives, together with "user intermediaries" who align various actors during the acceleration phase while increasing the amount of "user-citizens" who mobilize against the prevailing regime, hollowing it out, causing ever more consumers to choose the emerging regime. In this schemata, civil-society roles are clustered under the roles of user-citizens and user-legitimators, and direct engagement with technology falls under the roles of user-producers and user-consumers.

The invisibility of most citizen and user contributions, as discussed in Chapters 1 and 2, is a particular problem for transitions research. Transition studies are most commonly conducted based on document analyses, surveys, and expert interviews in order to cover the often long and wide change processes (Zolfagharian et al., 2019; Murto et al., 2020). But experts in industrial fields are seldom aware of the work done by users beyond individual instances, and thus users' activities tend to remain invisible to them (von Hippel, 2005, 
2016). Surveying tends to focus on actors seen as central in the industry, and it would indeed be difficult to target an undifferentiated mass of people for improvements without specifically devised representative surveys (De Jong et al., 2015). Some of the reasons for non-visibility can also be more blatant, such as supplier companies denying the primacy of functional user design due to its crudeness (von Hippel, 2016). All in all, while users attract much interest across disciplines, they are difficult to study systematically from historical materials or through other arm's length approaches; for instance, by running expert interviews at the supply-end or governmental agencies.

The above reduces transition research to a two-sided position regarding user contributions to sociotechnical change. On the upside, transition framing could integrate the diverse research streams on the forms of user contributions to long-term sociotechnical change. On the downside, the methodological grounds by which transition studies have been pursued may remain inadequate for the task, having a tendency to fall towards 'hollow' depictions criticized in chapter 2. BOAP research holds potential to improve the situation by providing both detailed and temporally extended research against which more site- and time-limited studies can be compared and against which theory building can progress without making sweeping model-based assumptions.

With this aim, we outline the Finnish heat-pump transition for the heating of detached houses (Section 5.2) and integrate the evidence given by the BOAP sub-studies for the roles that users have played, and we then give a detailed elaboration of each of the observed roles (Section 5.3). We proceed to present a more fine-grained model of local transition as a series of configurational movements (Section 5.4) and finally discuss the findings against previous theory building (Section 5.5). In doing the above we have complemented our studies with the historical analyses of heat pumps in Finland during 19782015 conducted by Heiskanen et al. (2017a) and Lauttamäki (2018), and other research into heat pumps (e.g., Heiskanen et al., 2011; Martiskainen, 2014). For more details on data and methods, as well as the Finnish country context, see the Appendix of this book.

\subsection{The heat-pump transition in Finland and evidence of user contributions in different phases, 1980-2018}

Heating of interior spaces in Finland has featured a varying mix of burning oil, solid wood, coal, gas, and peat, heating along with direct electricity, and the recent rise of heat-pump has produced additional energy. For residential detached houses, which comprise an average of roughly $70 \%$ of the present Finnish housing stock and which are the subsection of space heating in which we examine heat pumps, the last 50 years comprise a sustainability transition from the fossil fuel dominance of the 1970s (accounting for $80 \%$ of space heating energy, the other $20 \%$ being covered by the small-scale combustion of 
wood) to a "remnant problem" of the last $10 \%$ of fossil fuel heating in 2020 amid a more varied energy palette.

Out of the newly built detached houses, $50 \%$ are today equipped with a ground-source heat pump (GSHP) and an estimated 20\% have some form of air-source heat pump (ASHP) as the prime heating source $;^{1}$ district heating, solid wood, and direct electricity cover the rest. Heat pumps have also been the most popular retrofit option, and currently over 1 million heat pumps are in operation in Finland, both as primary and secondary heating sources in approximately 1.4 million applicable buildings, even though the heating retrofit cycles are slow and the transition will thus continue for some time to come. Along the changes in adoption the transition is associated with the changing of key production and retail-side actors from oil to heat-pump associated ones, changes in taxation, technology development, and other institutional changes. The development has been mostly market based, only minor household and energy renovation subsidies have been in place since the 2000s.

In a recent European comparison, Finland is among the countries, in which relative household energy prices favor electricity and heat pumps over gas and oil, and in many of which heat pumps have also diffused widely (others being NO, SWE, AT, CZ, LT, FR, PT, and NL) (EHPA, 2018). In contrast, in countries where gas, oil, or district heat prices have been consistently lower than electricity prices, and thus the overall heat-pump produced energy price has been less favourable, only a modest heat-pump uptake has taken place (GE, UK, BE, IT, DE) (EHPA, 2018). (See the Appendix for more details on the Finnish energy sector, energy policy, and market conditions for heat pumps.) These relative energy prices have been relatively consistent over the years and are good to keep in mind when considering comparability as countries with low gas and oil prices continue to feature an economic environment where the heat-pump transition is not helped by relative energy prices.

Finland was among the early-adopter countries of commercial heat pumps in the wake of the oil crises in the late 1970s and early 1980s (Heiskanen et al., 2014, 2017a; Lauttamäki, 2018). The early installations were GSHPs with horizontal heat collector systems on the land or in water reservoirs, built by plumbing and coolant companies that diversified into the domain. The early Finnish market development featured several companies, and by 1985 roughly 12,000 units had been sold to house owners and to various small and medium-sized businesses (Lauttamäki, 2018; SULPU, 2018). In the media, heat pumps were predominantly discussed positively, albeit they were the target of incumbent attacks during the course of the 1980s (Heiskanen et al., 2017a). The nascent industrial field featured little organization or domestic research, and various quality problems riddled many early installations. Yet the market developed positively with relatively competent pricing until the oil price decreased dramatically in the mid-1980s. Once the cost drivers for adopting heat pumps against the then-current and projected energy prices disappeared, the sales stalled and the field entered a death spiral. Companies gradually quit 
and maintenance and customer problems were left unresolved, leading to a tarnished reputation and the cessation of commercial activities by the 1990s (Lauttamäki, 2018; Lauttamäki and Hyysalo, 2019). All in all, this pre-development and nascent take-off did not alter the position of the oil-based and direct electricity heating regime in the Finnish residential housing sector.

There is scant research evidence on user roles in this early development period. Some users acted as advocates of heat pumps in public media and many more must have championed the new technology in their own buildings in order to have the heat-pump systems implemented successfully given the state of the product offerings available at the time. Most importantly, there were 12,000 early adopters whose purchasing decisions fueled the birth of the industrial field; yet, after the oil-crisis shock, the rest of the citizenship no longer followed.

The Finnish heat-pump field rose from the ashes in the mid-1990s owing to the continued expansion and development of the field, particularly in neighboring country Sweden (Zogg, 2008; Dzebo and Nyqvist, 2017). The heatexchanger equipment was now more standardized and reliable, the newly developed vertical borehole and collector technology made adoption possible for a far greater number of people and locations (as one no longer needed access to a water reservoir or a large land area) (Heiskanen et al., 2014, 2017a; Lauttamäki, 2018). The suppliers organized themselves under industry associations (the Finnish Heat Pump Association [SULPU] and the Finnish Borewell Association [Poratek]) in order to provide training, monitoring, public lobbying, and a voice in the energy field. Associated with steadily rising oil prices and environmental taxes on oil and electricity, GSHP installations started to re-emerge in the residential retrofit and newly built markets after 1995. At the same time, some older, early 1980s units began to be withdrawn (Heiskanen et al., 2014, 2017b; Lauttamäki, 2018).

ASHPs were also introduced as heating devices in the late 1990s, after being used for cooling in warm climates (Lundqvist, 2008). While there were doubts about the energy saving they would provide (Heljö and Laine, 2005), ASHPs were inexpensive, ranging from mere hundreds of euros to $€ 2000-3000$ (in contrast to the $€ 15,000-30,000$ cost of GSHPs), were more straightforward to install, and had a quick return on investment, even if they might not work well as a primary heating source mid-winter. ASHP sales rapidly increased to over 40,000 units sold annually since 2005. By the mid-2000s, heat pumps had proliferated to roughly $5 \%$ of the estimated 1.4 million buildings that they could apply to in Finland (Hyysalo et al., 2018).

In transition terms, the years from 1998 to 2005 are best periodized as a (new) take-off phase that gave way to an acceleration phase around 2005 (see Figure 5.1). In terms of indicators for the phases, technology development happened elsewhere to the extent that by 2000, both GSHP and ASHP systems were again cost-competitive in the Finnish market. Installation numbers rose rapidly among early adopters up to 2005, after which a market development stabilized to roughly 50,000 units sold annually. Adoption moved beyond early 


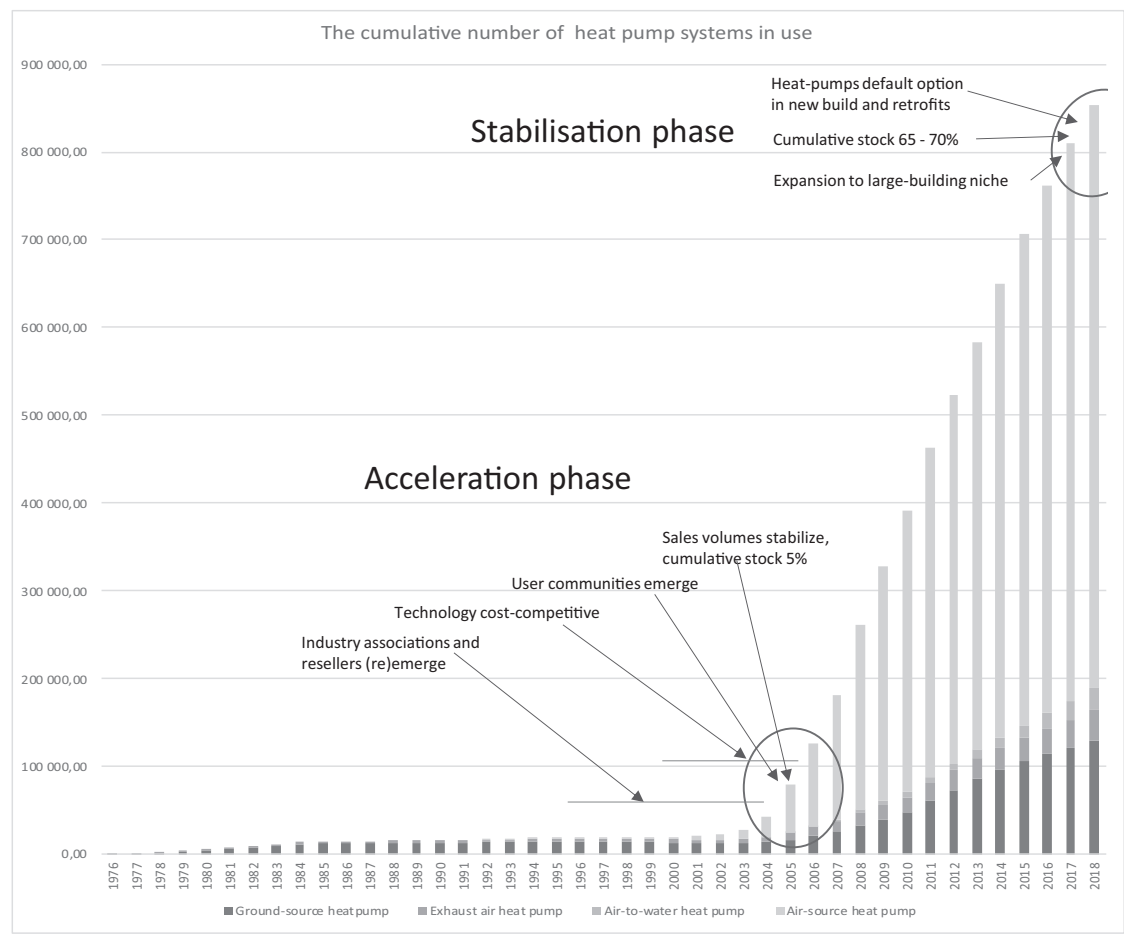

Figure 5.1 The cumulative number of heat pumps in use during the take-off and acceleration phases of transition. Source for the statistics on heat-pump cumulative amount: SULPU ry.

adopter segments between 2005-2010 for all heat pump types. In terms of institutional development, the industry associations emerged in the late 1990s, and amount of resellers rose as well, including the largest hardware store chains by the 2010s. User communities also emerged and changed, as we detail in chapter 4 . These aspects of the technological niche build-up followed each other so that a mature niche was in place around 2004-2007, with, as yet, few changes inflicted on the heating regime.

There is more evidence of user activities in the 1995-2005 period thanks to the archives of the peer-to-peer internet discussion forums discussed in Chapter 4. Some users championed installations and passed on information about heat pumps in their local networks. Internet forum discussions reveal considerable efforts by prospective consumers to assess the yield and payback times of GSHP systems due to uncertainties related to boreholes at the time. Also, installation, maintenance, and repairs were discussed among peers, showing intermediation in local settings and, with the spread of internet forums, also nationwide. Innovations by users in heat pumps became actively revealed in 
internet discussion forums following the introduction of first forums in 2004 and 2006, indicating that innovation activities by users were already underway earlier (Hyysalo et al., 2013a, 2013b, 2017).

Given the above we periodize that the acceleration phase in Finnish heatpump transition started between 2005-2007. While the annual installation numbers stabilized in 2006, the early acceleration phase still featured many uncertainties for both technology and the market, as discussed in Chapter 4. The trustworthiness of supplier information and the reliability of installers were still ambiguous, and making an informed decision was time consuming (see Chapter 4). Also, as late as 2010, some experts expressed doubts about whether ASHPs actually saved energy in real buildings due to the low coefficient of performance being further decreased by wall partitioning indoors. In contrast, toward the end of the acceleration period in 2017-2020, few such doubts or market difficulties were present. Purchase comparisons could be run in a matter of hours in internet forums, and the education and experience of the installers and resellers had improved. Resellers now included the largest hardware and electronics retail chains. The industrial field on the whole had become recognized and its industry association, SULPU, was active in field-internal development and national energy policy.

Also technology characteristics for cold country contexts had advanced for both GSHPs and ASHPs. For ASHPs the models for cold climates advanced to such an extent that a positive energy yield was retained with temperatures under $-20^{\circ} \mathrm{C}$, in contrast to the early range of $-10^{\circ} \mathrm{C}$ to $-15^{\circ} \mathrm{C}$. Also, new user practices had stabilized; for instance, it was now an accepted practice that many ASHP adopters kept their former primary heating system - be it oil, direct electric heating, or solid wood-and only used it for the lowest temperatures, whereas they used the ASHP for $80-90 \%$ of days in the heating period when its efficiency was good.

All in all, the uncertainties and ambiguities surrounding heat pumps in residential housing mostly dissolved, apart from use in very unusual settings. With GSHPs the drilling technology advanced and grew cheaper to the extent that boreholes could be extended cost-effectively to 300 meters, compared with the initial 60-80-meter levels, which allowed the scaling up of GSHPs to cover $100 \%$ of heating needs from the typical early $80 \%$ that left uncertainties about sufficiency. The Geological Research Centre of Finland modeled the ground-source heat potential across the country, aiding planners' and property owners' decisions about GSHPs (Lauttamäki, 2018). During the acceleration phase, both ASHPs and GSHPs became mainstream and were normalized in the Finnish heating market. Both benefited from increasing landscape pressure from climate change in terms of cultural values, and they also directly benefited from rising energy costs and taxes on fossil fuels. Heat pumps also benefited from neighboring niches, such as the proliferation of underfloor piping, that provided better efficiency for GSHPs.

Evidence of several user roles in the acceleration phase development is abundant. The most decisive action is the adoption of over 600,000 heat 
pumps, which in turn incentivized the resellers, manufacturers, industry association, and vocation training to invest in the developing heat-pump field. Many users were active in adjusting the technology to their particular houses, heating systems, and daily practices, and respectively adapting their heating practices and contexts to the heat-pump technology (Heiskanen et al., 2014; Juntunen, 2014b; Hyysalo et al., 2018). Also, DIY projects prospered, including next to 100 user innovations as we detail in Chapter 3 (Hyysalo et al., 2013a, 2013b, 2017). Some users in local communities, as well as in internet communities, acted as champions to aid heat-pump use and proliferation (Heiskanen and Lovio, 2010; Hyysalo et al., 2013b, 2018; Heiskanen et al., 2014), associated with the growth of user communities, out of which there is sporadic evidence of local communities playing a role in the early years of acceleration and strong evidence of internet communities playing a substantial role across the period (Hyysalo et al., 2018), also as user-side innovation intermediaries (Stewart and Hyysalo, 2008) who aid diffusion (Mignon and Bergek, 2016). Regarding market formation, and the purchase and selection of equipment, the internet discussion forums were important in storing and updating the technology, product, and market information in the changing field and in curbing substandard installations or misleading sales (Hyysalo et al., 2018).

In terms of periodization, the acceleration phase is characterized by the extending and gradually stabilizing resales points and offers, suppliers serving the field, media publicity, user practices and communities, research inputs, the beginning of vocational training, and the maturing of technology characteristics. Between 2006 and 2017, the heat-pump stock grew from 80,000 to 750,000 (i.e., from $6 \%$ to $55 \%$ of the maximal diffusion).

From 2017 and ongoing, the residential sector market's heat pumps have begun to enter the stabilization phase, where heat pumps become the dominant option in the market (see Figure 5.1). As noted above, by 2019 no less than $70 \%$ of new detached houses in Finland were equipped with a heat pump as the primary heating source. The replacement rate of oil heating in retrofits with GSHPs or air-to-water heat pumps also continues steadily, as do installations of ASHPs into direct electricity heated homes. The national climate policy targets rely on heat pumps substituting the last fossil fuel consumption in detached housing. In public media and everyday discussion, heat pumps in residential houses no longer appear as a novelty, and their further proliferation appears to proceed as a self-evident matter, sales remaining at a steady or rising level with new annual sales records appearing every now and again (SULPU, 2018).

The stabilization of heat pumps in detached housing is further paired with larger-scale heat-pump systems starting to take off in the large building and district heating markets (Lauttamäki, 2018), but as this niche concerns a different ecology of actors than the residential sector, we do not examine its development here. Regarding present user activities we see steady adoption, adaptation, and some DIY still displayed in non-standard housing contexts, as well as 
the intermediation taking place. The market and legitimacy characteristics are well developed.

All in all, the GSHPs and ASHPs have followed a substitution pathway (Geels and Schot, 2007), displacing oil and direct heating as the dominant heating systems in residential housing (Lauttamäki \& Hyysalo, 2019). A subset of ASHPs in hybrid heating configurations follow a reconfiguration pathway wherein they are added-onto the mix of existing heating equipment that is based on solid wood and remnant oil heating. The effect of heat pumps on the detached housing heating market has been that the formerly dominant fossil fuel-based heating, technology providers, and fuel suppliers have given way to the dominance of heat-pump and electricity-related technology and market players.

\subsection{Clarifying the user activities related to the Finnish heat pumps}

We now move deeper into user activities in the Finnish heat-pump transition. We elaborate them in more detail and better spell out their interrelations and importance to transition. We do so by departing from adoption and routine use, moving to adjustment and adaptation, and onwards to the championing of projects and innovation by citizens, all of which are primarily conducted by individuals, even if often helped by peers. We then elaborate on communities and intermediation, which take place both locally and digitally, and finally discuss market creation and legitimacy building, which appear foremost as the aggregate effects of large digital communities even though some of these activities take place without individuals being digitally connected. All in all, the Finnish heat-pump transition thus features a blending of the ways by which users' have been seen to shape the sociotechnical change covered by three generations of research we outlined in the introductory chapter.

Adoption and routine use are the most common and most important contributions that citizens make to advance transition. They do so not only as "consumers" making buying decisions but also as people sustaining the use of heat pumps and integrating heat pumps into their everyday living practices and sustaining a predominantly positively tuned discourse with the people they encounter. Heat pumps presented not only a new heating technology but also a new type of warmth. This is most pronounced with ASHPs that rely on an air convector to transmit the warm airflow into space. There is audible noise from both the convector and the outdoor collector unit that is associated with the periodic burst of warm air, which stirs indoor air into motion. The air typically needs to be blown from above to effectively spread into the space. The distribution of heat through air mixing is a different type of warmth than that which Finns are accustomed to from their earlier heating forms. Solid wood stoves or radiators below windows (be these water or direct electricity heated) provide point heat and radiation, which is typically experienced as pleasant and, in most 
cases, is distributed around the house in such a way that closed doors or other partitions do not affect it. It is not trivial that such new conditions of comfort are adopted and accepted in everyday living and that positive word of mouth and public discourse accompanies them. GSHP systems were more compatible with radiators or underfloor heating, but for these systems adopters faced a considerable $€ 10-20 \mathrm{k}$ investment and, in the early phases, still needed to trust a system that scaled to $60-80 \%$ of the maximum heating need in order to save in the upfront costs.

We have also stressed that even before people got to experience heat-pump usage, they needed to invest time and effort into the purchase process-scaling, comparing, selecting, planning, and overseeing installation-and the effort required for making an informed choice was considerable in the early stages of heat-pump proliferation. It could have taken days or weeks of part-time work, depending on the thoroughness of the decision and the particularities of the installation. On top of this, even routine use requires occasional maintenance.

Adoption and routine use also concern by far the greatest population: with over a million installations, heat pumps touch the lives of a third or even half of the Finnish population of 5.5 million. The simple adoption numbers also concern the greatest leverage point for the transition: the investment that has gone into the Finnish heat-pump field to date is $€ 4$ billion, which has enabled the 4000 people in supply, retail, installing, maintenance to work in the field and to improve their operations. It has further legitimated energy counseling, educational programs, and (modest) tax deductions along the way. The simple purchase amounts have also been a clear signal to heat-pump manufacturers who have further developed their offerings to better match cold-climate contexts with significant improvements in the two decades of heat-pump transition in Finland.

Yet, as we have argued, adoption has not been automatic and has been importantly supported by other user activities, particularly with respect to moving heat pumps from early adopters to later ones, who were predominantly less motivated by environmental choices or technical novelty and had less interest and ability to conduct their purchase and adoption decisions. Adoption remains modest in early transition stages and the rise in sales number was associated with other, arguably more intensive, user engagements with new heat-pump technology.

Adjustment and adaptation have been common in getting routine use going. Most users have had to make some changes to their daily habits regarding, for instance, keeping room doors open, changing furniture layout, or changing routines related to emptying iced-up meltwater from the heat pump. Installing the heat pump in a particular house is typically also a process where adaptations and compromises have to be made regarding the aesthetics of the indoor space and the outdoor appearance, and the effectiveness of the heat emanating from the convector unit (Heiskanen et al., 2014; Hyysalo et al., 2013b; Jalas et al., 2017). Notable discrepancies between factory values and the yield in real, site-specific use at different temperatures have been 
presented by resellers and have prompted many users to monitor their equipment more carefully and to consult their peers and resellers regarding if and how the yield can be improved through changing the settings or through small hacks (Hyysalo et al., 2013b, 2018).

Evidence of adjustment and adaptation can be found in all transition phases (see, e.g., Motiva, 2010, 2012; Hyysalo et al., 2018; Lauttamäki, 2018), but to estimate its proportional incidence in different transition phases and the expense of time and effort used on it would require devising a representative survey (akin to the survey conducted by De Jong et al., 2015) or a representative wide-scale interview study on heat-pump users, administered at a set time after implementation and updated longitudinally (akin to the study by McLaughlin [1999] but applied to hundreds of people with an arguably difficult-to-establish sampling frame).

Innovation by users is documented from the take-off and acceleration phases. As documented in Chapter 3, hundreds of DIY projects were pursued by consumers, and while over 400 of them were verified as having some novelty and benefits by domain experts, 113 of these projects turned out to be newto-the-world innovations (Hyysalo et al., 2013a, 2013b, 2017; Mattinen et al., 2014). As noted, these innovations mostly emerged as responses to local particularities, which remain poorly catered for by commercial models that seek to appeal to common denominators of wider user populations and thus, for instance, sacrifice potential energy gains for the sake of added safety and reliability. The user innovations introduced solution variety, both to technological offerings and new uses, but their verifiable diffusion, and thus their realized variety, remains more modest: in Finnish S-RETs, 2.7\% become commercialized, $8 \%$ are copied by peers, and $34 \%$ are fed into further DIY projects as a part of further solutions (Hyysalo et al., 2015, 2017). Yet, as discussed in chapter 4 user innovations have had another important contributory capacity: the innovation and DIY projects and the peer-to-peer knowledge exchanges that accompany these built in-depth competence and networks among this set of enthusiasts then fed into user intermediating (Hyysalo et al., 2013b, 2018). User innovations can be found from the take-off phase to the late acceleration phase.

The championing of ambitious projects, in community building and in the dissemination of information by users, happens from the exploration phase to the acceleration phase. Project championing (Klerx and Aarts, 2013) must have taken place in early pioneering projects that were novel and also demanded considerable effort from the adopters (Lauttamäki, 2018). In later phases, technology championing is seen in efforts to introduce heat pumps into new non-standard installations, in the considerable efforts by users to assess the yield and payback times of both ASHP and GSHP systems (in particular, their settings and geographic zones), and in running jointpurchase projects (Heiskanen et al., 2011; Hyysalo et al., 2013b, 2018; Hyysalo and Juntunen, 2018). Championing intermediation (Martiskainen 
and Kivimaa, 2018) took place in the internet communities, where a group of roughly 500 people provided tens of thousands of responses to various queries from others. It is important to note that none of these efforts are necessarily advocating heat pumps but geared to serving oneself and helping others on the side.

Community creation and maintenance have been an important catalyst for other user activities. There is sporadic evidence of local communities playing a role in the exploration and start-up phases, while the internet communities played a substantial role during the acceleration phase. A key feature here has been the accumulation of a critical mass of community members and information that has accumulated in such a way that the internet communities have served the varying needs of prospective adopters and existing users. In the Finnish heat-pump transition there was the coincidence of the emergence of the first tens of thousands of users with the emergence of easy-to-set-up discussion platform technology (Hyysalo et al., 2018). The later diffusion of solar PV and solar heat in Finland showed that internet communities could play a role from the early take-off phase, but the critical mass has been slow to cumulate (Hyysalo et al., 2017; Hyysalo and Juntunen, 2018). Regarding locality-based communities, there have been few community energy initiatives or joint purchases that are significant for heat pumps; yet interactions in local communities where somebody has adopted a heat pump have been conducive for spurring further adoption among peers; for instance, within carbon-neutral municipalities (Heiskanen et al., 2017b).

Intermediation by peers comprises not only facilitation but also the configuration of equipment and brokering of connections (Stewart and Hyysalo, 2008). Intermediation has a dual form in the Finnish heat-pump transition: first, as local peer-to-peer encounters and, second, as an aggregated many-to-many phenomenon, amplified by internet communities (see Chapter 4). In local sites, intermediation takes place from at least the takeoff phase onwards (Heiskanen et al., 2011, 2017b). The intermediation by users intensifies in the peer-to-peer communities, which facilitates learning about new technology and its use among peers. The accumulated display of exchanges about installations, use experiences, real-time monitoring, technology concerns in the cold-climate context, and DIY solutions also contributed to demand articulation and technical improvement (see Chapter 4). In internet communities, direct peer designing was rarer as the distances between users prevented peers visiting each other(cf. Meelen et al., 2019), but help with configuring equipment was abundant, as was help with the brokering of contacts.

Market creation activities are not unidirectional supply-led initiatives that spur demand-they form an interactive process in which adopters take part. One aspect of this is that adopters spur their peers on with buying decisions by providing examples and testimonials as to the worth of the goods. The other aspect is that in order to act in the market, potential adopters need to be able to make 
sense of the qualities of products, compare them, and establish an understanding of what consequences different choices hold for them (Callon et al., 2002). Both these aspects of market creation were central to the intermediation that users provided and particularly in the internet communities (see Chapter 4). The internet discussion forums were particularly important for storing and updating the technology, product, and market information in the changing field during the acceleration phase. They also indirectly policed supply and retail actors through fostering brand- and make-specific discussions that highlighted problems, such as erroneous supplier claims or substandard installation services (Hyysalo et al., 2018).

Legitimacy building is a key process in how a new technology becomes intertwined in the fabric of society (M. Suchman, 1995; Janasik, 2011), and there is evidence of this happening in the acceleration phase of Finnish heat pumps. The early stages of a new technology are characterized by uncertainties, doubts, and counterclaims to its proponents' narratives (Phaffenberger, 1992). Such "technological drama" is the overtly politicized side of sociotechnical change, wherein citizens and citizen movements play an active role (Williams and Edge, 1996; Smith, 2012; Schot et al., 2016). Adopters can, however, also play a key role in the regularization of technology with less sound and fury. As presented in Chapter 4, the internet communities fostered and made visible a positive legitimating discourse about heat-pump technology. While forum discussions feature plenty of critiques, they also feature a conspicuous absence of wholesale doubts or dismissals of heat pumps, generic technology critique, or even the sustainability-related ideological discourse typical of grassroots innovation and critical niche communities (Smith et al., 2014). Such taken-for-grantedness may well be the most powerful aspect of technology-related discourse, and when it was displayed in over 300,000 messages that attracted over to hundred million reads, the effect was considerable.

To clarify the interrelations between the above-described user activities and their importance to understanding transitions, we proceed through two sequential diagrams. The first diagram (Figure 5.2) presents the actor-level interrelations between the activities of the users and how they link to niche, regime, and landscape actors. These relations can be observed from the takeoff stage onwards in the Finnish heat-pump transition. Let us first clarify the basic interrelations. Adoption and a growing market foremost signal user activities to other niche actors and to users still aligned with the existing regime (the dots with horizontal lines in Figure 5.2). Among the adopters, some champion new types of projects as well as peer interactions in local and internet communities (the ascending line dots). Some adopters must make adaptations and adjustments (the descending line dots), and a small subset of these people proceed to DIY projects and further user innovations (the checkered dots) as well as to user intermediaries who help others (the dots with grid). The user innovators affect other users through three mechanisms: by adding to the solution variety available to adopters and adapters, by 

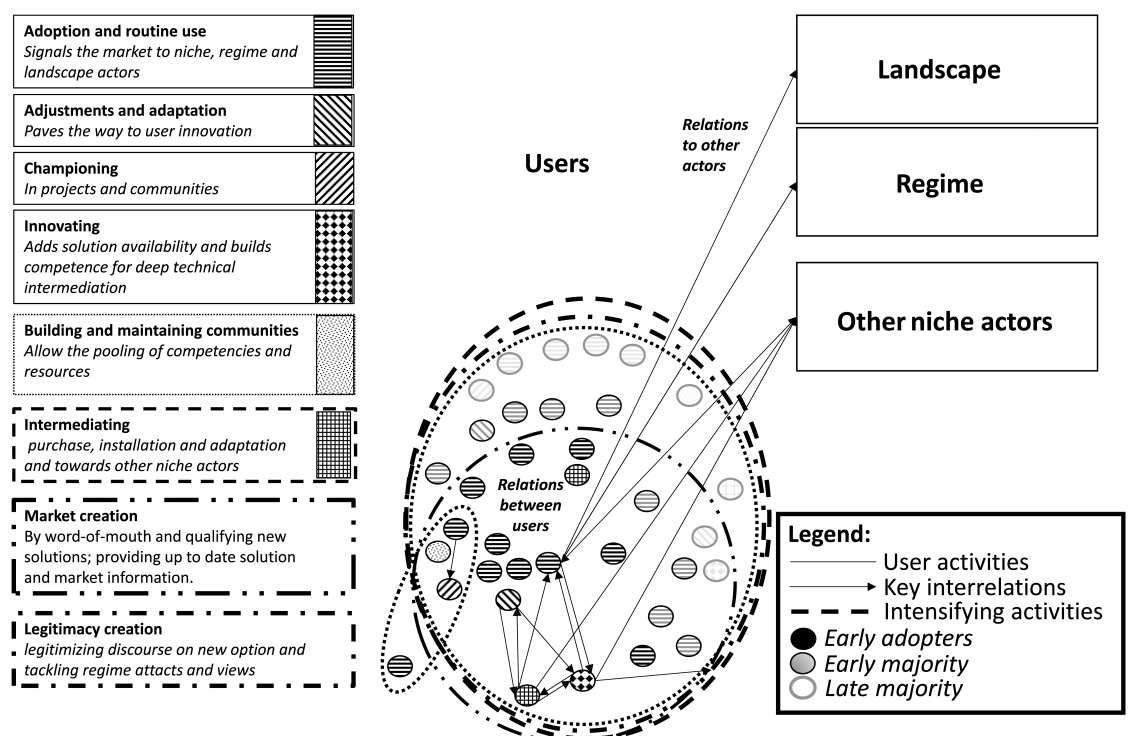

Figure 5.2 The actor-level relationships of user activities in Finnish heat-pump transition.

signaling designs and design needs to resellers and manufacturers that serve the niche, and by providing technical and practical competence in intermediation in communities, allowing these to deepen into useful repositories of knowledge and encouragement (the dotted circles) that help mediate the other activities.

The actor-level depiction of user activities needs to be complemented by adding to the depiction activities that emerge and intensify when the adopter base grows and becomes connected through internet communities. These included a wide intermediation among peers that was not confined to just a few locations (the dashed-line circle), market creation (the dash two dot circle), and the creation of legitimacy through widely read regularizing discourse (the dash-dotted line circle). The intensification of these user activities affects the other niches, regimes, and landscapes to varying extents, but particularly the not-yet users who are still aligned with the regime but gradually rethinking their heating solutions.

Figure 5.3 aggregates the actor-level findings to an overall transition-level depiction for comparison to earlier schematics by Smith (2012) and Schot et al. (2016). The complex interrelations between user activities have been suppressed in favor of portraying the user activities in the course of the transitions, a common facet in transition models that seldom address the actor interrelations in detail. 


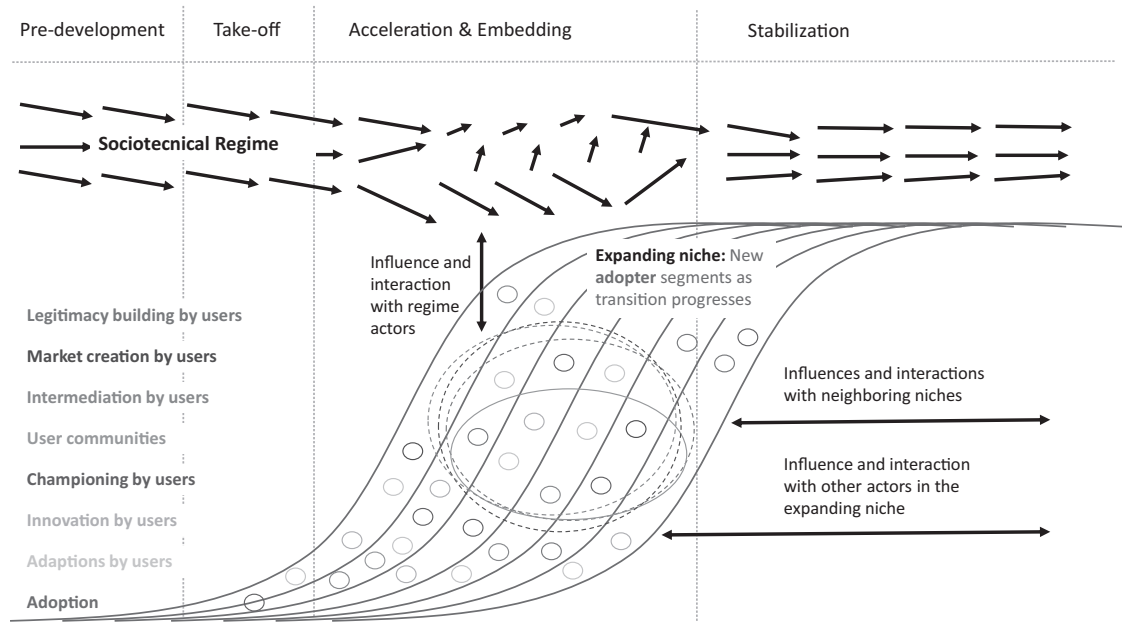

Figure 5.3 User activities presented at the transition process level.

The key features in Figure 5.3 are the emphasis on the technology expanding into new adopter segments in the course of the transition, which is aided by user contributions to adaptation, innovation, championing, communities, legitimacy creation, intermediation, and market creation. The user activities have influences on and interactions with other actors in the expanding niche, with neighboring niches, and with regime actors.

Importantly, all the user activities depicted in Figure 5.3 have incidences across the transition and we refrain from making assumptions about the relative importance of different user contribution types in different phases. We place legitimacy building, market creation, and intermediation highest up on the list as they intensify the most in the Finnish heat-pump transition (dashed wider circles in Figure 5.3). This is because isolating the relative importance of different user contributions is not supported by our data and may in fact be methodologically impossible because of the different mechanisms of influence among tens of thousands, and later hundreds of thousands, of users. (See the chapter conclusions for an extended discussion on this point.) The mutual influences are better portrayed in an actor-level model.

\subsection{Understanding transition as a series of configurational movements}

To render transition studies more capable of addressing the specific phenomena of interest within the overall systems change, more fine-grained theoretical registers may be needed as already hinted by the difference between actor and transition level models of user activities 5.2 and 5.3. In this book such a register 
is pursued through ecological views of sociotechnical relations, particularly the concepts of arena and ecology actors introduced in Chapter 2 that help trace local transition as a series of configurational movements (Hyysalo et al., 2019a). These movements amount to "key biographic moments" in the change process studied (as worded by Glaser et al., 2020). In these moments the relationally constituted character of a technology, its material make-up, underlying principles and theories, designed artifacts, and the practices of associated people are shifted. In previous BOAP studies, four typical moments and configurational movements can be discerned in the life-cycle of a technological artifact: constructing the problem, selecting and developing a solution candidate, deploying and using the technological assemblage, and reusing and translating the technology in other locations (see, e.g., Hyysalo et al., 2019; Glaser et al., 2020; Hyysalo and Usenyuk, 2015). For our interest here, however, the picture is somewhat more complex as sociotechnical change in energy transition takes place at the scope of a technology field where several biographies interlink and produce aggregated effects (Pollock and Williams, 2008; Hyysalo, 2010). We thus need to traverse at once the key changes of particular sociotechnical assemblages, as well as the key changes at the aggregate level. Particularly in regard to this latter interest, the concepts of the ecology of actors and arenas become useful, and let us concretize these concepts with the example of early GSHP proliferation in Finland.

By sociotechnical configuration we mean the intertwinement or "assemblage" of those technical and social elements that produce outcomes in an identifiable setting (Latour, 1987, 2005). A simplified example of sociotechnical configuration in heat pumps is, for instance, a "horizontal GSHP system." It is comprised of the following:

- a horizontal collector GSHP, the requisite plot of land, and land-moving equipment

- the design choices made by the equipment manufacturer and its component providers

- the sales arguments, pricing, delivery types, and resellers

- installation practices by installers and adopters

- the adopter practices in the running and maintenance of the GSHP unit and its collector field; the re-landscaping of the collector field

- the management of heat intake

- heating needs and other heating solutions by the adopter

- the management of winter ground frost by adopters.

This non-exhaustive list underscores that the "artifact" is never the center of attention nor the defining point of analysis as such, but always exists in a "contexted" way in particular settings where the artifacts are enacted and consequential, as Glaser et al. (2020) put it.

The elements of configuration, be they "technical" or "social" at a particular site of configuration, are bound to actors through the materials, designs, principles, theories, usage, and regulations that are enacted 
(Hyysalo, 2010) and may imply other actors and actor behaviors. For instance, a horizontal collector field for a GSHP implies an adopter who can lay hundreds of meters of coil in the close vicinity of his house without being disturbed by the effects on his house surroundings, including the prospect of potentially having to dig the ground up anew to mend problems that may occur with the coil.

But sociotechnical configurations around particular installations that are bound to and imply actors also point to a wider ecology of actors that bears an effect on the technology type-typically consisting of tens of competing makes and models - in a particular arena in which these actors have their dealings (that may involve several types of technologies at once) and through which the arena is defined among the different social worlds present in it through the involved actors (Strauss, 1978; Clarke and Star, 2003; see Chapter 2). The sociotechnical configurations related to an ecology of actors and to an arena are typically multiple, and each implies a subsection of the actors in the area, while their effects typically reach further in the arena. Once the technology type and markets mature, the result is a relatively stable industrial field. But because the field organization is the long-term result of sociotechnical change rather than its pre-condition, the ecology of actors and arena concepts are more apt for discussing it than using the term "industrial field" until there is a field that has matured into existence.

By configurational movements we mean significant shifts in the configuration, typically comprised of new actors, materials, practices, regulations, or competencies becoming intertwined in (or replacing) the previous relations. A common way this happens is that the site and situation for the configuration change so that they become tied to a new actors that were only implied earlier (and conversely, previous ties may loosen up). To give a simplified example of GSHP systems at the scale of a technology type: Once vertical borehole design and installation solutions were developed, they not only expanded the user base to new types of peoples, houses, and allotments, they also tied the borehole drilling companies into the ecology of actors (largely replacing land-moving operations) and further changed the material makeup of GSHP systems, their installation competencies, and their principles as it became paramount to be able to estimate how deep a well would provide the needed heat yield in different types of ground (Lauttamäki, 2018). At the scale of a technology type, the above movement forms a part of a configurational movement wherein the heat-pump field became reorganized with new types of heat-pump products, resale operations, and the establishment of industry associations for heat pumps and drilling operations (see the below Movement Number 3).

With these clarifications in place, we can recount the Finnish heat-pump transition at the level of the emerging technological field through nine major configurational movements. The first key arenas and configurations for all S-RET systems resided outside Finland in the 1970s, when the basic heatpump, solar PV, solar collector, pellet burner, and micro-wind designs first 
gained commercial introductions and early markets. The ecologies of actors in these arenas typically featured experimentation by researchers, (typically modest) commercial producers, and users alike (see, e.g., Smith, 2014; Heiskanen et al., 2014; Lauttamäki, 2018; Nielsen, 2016).

With Finnish heat pumps, these international arenas led to the rise of a local heat-pump arena (second configurational Movement) in the early 1980s with 12,000 adoptions that emerged in response to rising oil prices and company offerings of the early GSHP and water source heat-pump technology. As noted the ecology of actors was comprised of small resellers and producers (or the small sub-units of larger companies) and early adopters who proceeded to purchase and then run (and struggle with) their installed systems. As described above, this configuration fell apart once fossil energy prices decreased and new purchases stalled, forcing vendors out of the market, leaving users to grapple with their systems as best as they could, and leaving in place little more than general skepticism toward heat pumps.

The next Finnish arena for heat pumps, emerging in the late 1990s, was equally pre-configured through the international arenas of both production and use that had made heat-pump technologies more developed: ASHPs for cooling purposes and GSHPs in heating through vertical borehole technology (the third configurational movement). This time the local arena had a more organized ecology of actors that featured not only resellers, installers, and small manufacturers but also featured two industry associations that salvaged the hard-won insights from the first configuration and its failure. By 2005, 60,000 installations had accrued, yet the heat pumps in Finland proliferated against the grain of country specifics, market conditions, and institutions (Heiskanen et al., 2017a; Lauttamäki, 2018; Lauttamäki and Hyysalo, 2019). This Finnish configuration (the fourth configurational movement) was thus, from the user side, still centered around early adoption and the achievement of routine use, shrouded by uncertainties as to the technology yield, savings, payback times, and scaling, as well as to vendor and installer trustworthiness. The adoptions required competencies in understanding what the new technology is and how it works, its potential suitability, possible permit issues, cost-benefit calculations, comparisons between suited makes and models, installation types, and the possibilities and requirements for routines, use, maintenance, and monitoring.

Thus the fifth configurational movement in the same arena and ecology of actors: sales and adoption were not only important, many users undertook adaptation, championing when they faced the need to alter the equipment or their social and technical contexts (physical houses, daily practices), and also their networks in regard to finding professionals to do non-standard installations and clarifying permits etc. This configurational movement among users was often invisible to other actors in the ecology. Here new competencies and materialities were highlighted as the technology and its context were no longer just bought and operated but tinkered with and adjusted in order that they work in a non-standard manner. 
The sixth configurational movement emerged with small subsets of people further improving their equipment through DIY projects and user innovations. This called for radically deeper engagement with the technology and the competencies to deal with it, including new kinds of peer exchanges. The adaptation and innovation activities changed heat pumps from a novel and difficult-to-understand black-boxed technology to a malleable good that could be adjusted and improved. These activities also affect other users through adding to the solution variety available to other adapters as well as signaling further design needs to resellers and manufacturers. As noted, people pursuing DIY and user innovation turn out to provide deep assistance in user communities.

The seventh configurational movement took place through new market and intermediary actors entering the ecology of actors, namely large hardware retailers and the build-up of internet-based user communities. The initially modest peer-to-peer knowledge-sharing platforms rapidly grew into popular, diverse, and deep repositories of knowledge in just five or so years, contributing to the intermediation, market creation, and legitimacy of the new technology and affecting the not-yet users aligned with the regime but gradually rethinking their heating solutions.

The eighth configurational movement was the mass adoption associated with the maturation of both the Finnish residential heat-pump arena and the stabilization of its ecology of actors, leading to increasing normalcy of residential heat pumps in resales, regulations, and public and professional media, as well as in adopter choices. The second decade of heat-pump proliferation after the restart (2006-2015) featured over a tenfold growth, which has continued since. This growth was not automatic, as it may appear to be from just examining the numbers, nor a simple result of an effective industry association (Berninger et al., 2017): it was paired with and made possible by a gradually structured market, knowledge institutions, and technology characteristics to which all of the seven earlier configurational movements contributed. The technical configuration gained further support from neighboring technologies, from generally improved insulation levels, and from the increasing adoption of underfloor heating that supported GSHPs' and ASHPs' capacity to act in conjunction with existing heating technologies and other S-RETs in residential houses.

The ninth and ongoing configurational movement concerns the present breakthrough of industrial-scale heat pumps in the large building market and as part of district heating networks. Owing to advances in large-scale heat-pump systems, the growth of some Finnish suppliers serving the residential housing GSHPs, and the availability of ground heat-potential modeling, these companies could start making competitive bids for heating larger installations that had previously been won by bespoke fossil-heated power generation or district heating networks.

This series of configurational movements provides a lens through which to view the specifics of how a new technology becomes socially and materially shaped in the course of sociotechnical change-it reconnects the user activities (Figure 5.2) into the dynamics of sociotechnical change in a manner 
that helps to focus on the situations and dynamics that matter the most. All the nine sociotechnical configurations feature some interactive dynamics between the supply side and demand side and intermediation processes, but Configurational Movements 3 and 8 are owed mostly to the actions of the supply side and Configurational Movements 5, 6, and 7 to actions taken on the demand side, in such a way that Configurational Movements 3, 5, and 7 imply a field-organizing intermediating move from the position of users or suppliers.

Innovation and sociotechnical change processes are known to feature considerable contingencies and idiosyncrasy among repeating patterns (Russell and Williams, 2002). Abstracting from the above analysis, we suggest a tentative set of abstracted configurational movements that are likely to occur in the course of a technology type becoming successful in a follower country of a transition technology (see Figure 5.4):

1) The search for principles of basic solutions and their design take place elsewhere (supply, demand)

2) Initial domestic introduction and adoption form a nascent ecology of actors (supply, demand)

3) Adaptation, championing, and user innovation take place to better suit the technology to the consumer specificities in the country (demand)

4) The build-up of sufficient intermediation takes place and lays ground for the emergence well-working domestic market (supply, demand, intermediaries)

5) Further technology and global market development feed into the development of the domestic market (supply, demand)

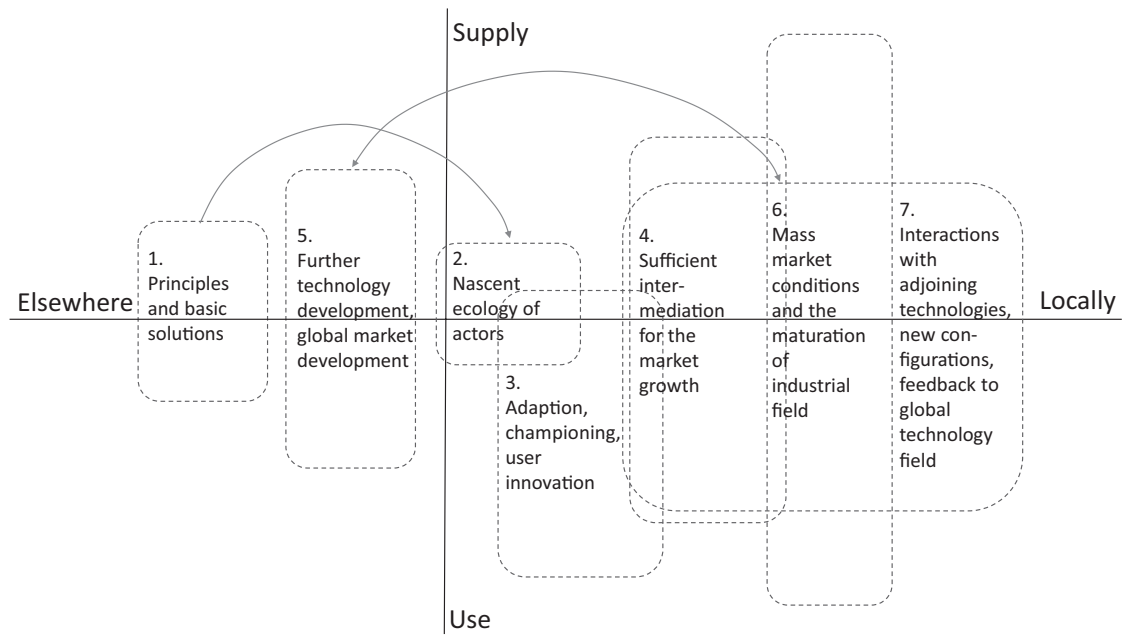

Figure 5.4 A configurational model of technology proliferation in follower contexts. 
6) The emergence of mass-market conditions and the maturation of the industrial field (supply, intermediaries)

7) Interplay with adjoining technologies, the emergence of new sociotechnical configurations, and feeding back to the global technology field (supply, demand, intermediaries).

\subsection{Chapter conclusions: the yield to and from transitions research}

Users' engagement in the shaping of new technology presents a well-established research area for several disciplines. Sustainability transitions research stands in a dual position vis-à-vis this earlier work. On the one hand, it presents a perspective that may have the potential to integrate and cross-examine a range of the citizen and user contributions that take place in the course of longterm sociotechnical change - a capability of obvious importance in the face of the compartmentalization of user-related research in innovation, diffusion, consumption, design, informatics, social movement, and S\&TS (Hyysalo et al., 2016a; Kohtala et al., 2020). On the other hand, the research on user and civilsociety influence on sustainability transitions has only recently begun to move beyond the more narrow framings of these earlier lines of study, leaving a research gap between overall appraisals and the detailed studies on users within sustainability transitions (Heiskanen et al., 2014; Schot et al., 2016; Kanger and Schot, 2016). Our investigation into user activities related to Finnish heat pumps combines historical and ethnographic studies and integrates multiple topical sub-studies, and it is also the first empirical analysis that is detailed enough to be likely to highlight a considerable part of the invisible work carried out by users, as well as to cover the majority of the transition from the beginning of the transition to the late acceleration phase.

The findings support examining the import of the manifold contributions that users make throughout the transition process without findings becoming siloed into different disciplinary discourses (Smith, 2012; Schot et al., 2016). Described at the actor level (condensed in Figure 5.2), our study reveals a richer set of users' active engagements and provides a more nuanced understanding of their interlinkages in the process of sociotechnical change than has been provided before.

The protracted spread of solar power, wind power, and heat pumps, and the use of micro-scale biomass despite price parity have made it clear that there is more to understand about adoption than economic and institutional development. The transition solutions move from ideally suited sites and locations to less ideal ones (Meelen, 2019; DeWald and Truffer, 2012), from early consumer segments to followers, and from early institutional contexts to those pertaining to mass adoption (Ornetzeder and Rochracher, 2013; Nielsen, 2016).

In our analysis, adoption and the consequently growing market are the prime mechanisms that signal user activities to other niche actors, as well as to the regime, landscape, and potential users still aligned with the existing regime. 
Adoption is, however, importantly facilitated by a range of other engagements with the niche technology. Adaptation and championing ensue when subsets of users face the need to alter the equipment or their social and technical contexts (physical houses, daily practices), and also their networks, for instance, in finding professionals to do non-standard installations and clarifying permit issues etc. Small subsets of people further turn to improving equipment (or saving costs) through DIY projects and user innovations. These activities affect other users through adding to the solution variety available to adapters, signaling further design needs to resellers and manufacturers, and by providing deep-level competence for intermediation in user communities, allowing these to build up into major repositories of knowledge and encouragement. Our analysis further suggests that user communities are conducive to playing a role in market creation, may provide wide and easily accessible intermediation among peers, and contribute to building legitimacy for novel technology. This intensification pattern is clear in Finnish heat pumps and has significantly affected not-yet users aligned with the regime but gradually rethinking their heating solutions. A transition-length depiction (Figure 5.3) underscores the continuation of adoption across different adopter segments and interactional effects between user contributions and other niche actors but downplays the user activity interrelations for clarity.

In articulating the actor- and transition-level contributions by users and their interrelations, our analysis stands in some critical contrast to earlier studies and theoretical proposals on users in transitions. The first of these concerns the assumedly politicized nature of transition. The transition in residential heating in Nordic countries, in which heat pumps have been a key technology, has been characterized as a "silent revolution" (Johansson, 2017), and as a corollary to this its user-side features remarkably less politicized civil-society activities than transition models and previous studies of citizen engagement have put forward (Smith, 2012; Ornetzeder and Rohracher, 2013; Schot et al., 2016). Similarly to solar PV, solar heat and biomass use, heat pumps in detached houses can mostly be installed through direct substitution without wide system changes, yet still instill wide systems change, as is evident in the defossilization of Finnish space heating in detached housing and the associated change in the actor groups involved in the field.

The second contrast concerns distinct-phased contributions by users. Our data supports the idea of the intensification of some user influences through the emergence of much-read internet communities during the acceleration stage but not a stylized grand transition narrative where certain user functions or roles would be dominant in certain phases toward not-yet users aligned with the regime or toward other regime and landscape actors.

The extended time frame of study also questions some assumptions made in other fields studying users. Even though user innovation has elsewhere been found to be most concentrated in the early development phases of new technology types (von Hippel, 2005; Franke et al., 2006), in Finnish heat pumps it continues long into the acceleration phase (Hyysalo et al., 2013a, 
2017) — this may well have happened in other small-scale energy technologies yet escaped attention as only the emblematic early stages have hitherto been systematically studied (Ortnetzeder and Rohracher, 2006, 2013; Nielsen, 2016).

Third, our study underscores issues regarding the terminology used in characterizing user activities and their emergent effects. We find it is sensible to talk about champions and legitimacy building rather than advocates and legitimators (Schot et al., 2016; Kanger and Schot, 2016). Championing is evident in our data, but active and important advocacy is less so, beyond isolated instances. Similarly, the hundreds of thousands of moderated posts and 200 million reads in internet communities aggregate into a legitimating discourse, while few discussants actively pursue legitimation. The terminology used is consequential regarding what demand-side dynamics and empirical phenomena researchers pay attention to, whether one underscores individuals as role holders or whether one directs attention to their activities and possible aggregated effects - for instance it would be illusory to incentivize people into better filling an assumed role, when the "role" turns out to be an aggregate side-effect of the factual activities they pursue.

Finally, our analysis suggests that there is a need for recalibrating transition research for the acceleration phase and invisible work by actors. Most of the evidence for transitions has been amassed from globally studying new niches in the settings where they have first occurred. These globally early settings, as important as they are for creating the alternatives that may then spread to other contexts, may in fact be rather poor model organisms for how the majority of the localized transitions are likely to play out after take-off. The late-mover settings interface with the alternative solutions that are already in a more developed phase technically regarding commercialization, distribution, and usage patterns. At the same time, the late-mover settings are likely to feature characteristics that differ from the early development contexts and which may have barred the early proliferation in the first place. These may relate to geographical conditions, competing technologies, the institutional environment, market conditions, or issues of wealth and inequality (and so forth). Finnish heat pumps are a good example of a transition process that (re)started after a death spiral at the point when both GSHPs and ASHPs had already passed their early development phases in other countries. This contributed to their fast proliferation in Finland, but this has not meant a smooth or inevitable diffusion as we detail above. In comparative terms, the relative global position is likely to result in somewhat different patterns, for instance, in the speed of the acceleration phase, the nature of institutional development, and in public discourse, as well as in the user activities in the transition. Tracing the series of configurational movements in heat-pump transition identifies which concrete dynamics to look for and provides points for advancing transitions on the ground. A minimal set of seven abstracted configurational movements in a follower country point to the likely dynamics and shifting locus of action in the shaping of sociotechnical change (cf. Williams and Edge, 1996). 


\subsubsection{Methodological considerations: Recalibrating transitions research}

While some transition scholars slant S\&TS as being too inductive to yield generalizable models, the BOAP investigation not only suggests that this depends on how S\&TS is carried out but also highlights the practical and principled difficulties in "testing" broad transition models. Because of the long temporal span and wide scope of the empirical domains involved, mustering direct reliable evidence in support of or against the general transition models may prove elusive. This is particularly so as they feature such key explanatory constructs as "rules," "learning," and "interactions" and actor groups like users and intermediaries. These are phenomena and actors on which empirical data is seldom systematically recorded and regarding which also indirect evidence such as time series kept by statistical bureaus or industry associations tends to be lacking. ${ }^{2}$

User contributions to transitions are a good example of the methodological issues involved because of the three senses of the invisibility of their contributions and the sheer numbers of users as the transition progresses, and allows us to discuss which of the key constructs of Smith (2012) and Schot et al. (2016) find support, which do not, and which are most likely impossible to verify.

First, there are general-level assertions in both the models of Smith and Schot et al. regarding users and consumers. These are assertions that transition progresses from early, underdeveloped technology, market, and social characteristics where adoption is a pioneering act to a point where it has become more natural and legitimate for consumers to make choices favoring the new regime; that users and civil-society actors play a significant role throughout the transition process; and that they do so in several ways beyond just adoption and financing. All these three assertions find ample support from the Finnish heatpump transition and our investigation clarifies further the mechanisms through which the various user contributions have taken place across one transition. So, our study could be taken as largely confirmatory of these general-level assertions. This said, these assertions reside at such a high level of abstraction that their value to understanding transition and users remains limited-users and civil society matter across the transition, and then what?

Regarding the more specific assertions regarding what, how, and when users and civil-society actors contribute, we should first acknowledge that Smith rather provides an illustration of how various civil-society formations may have an effect on transition processes and this we believe to be a sound way to link piecemeal research evidence to high-level transition models. In contrast, Kanger and Schot (2016) have proceeded to use the model of Schot et al. (2016) as a theory to be tested and it thus makes sense to discuss their constructs and their verifiability in more depth.

As noted in the introduction to this chapter, their core construct is the relative importance of five user contribution types in different transition phases. In Finnish heat pumps, all these user activity types can be found in all transition phases. But it remains unclear why just these five types would be important as the Finnish heat pumps alone surface eight salient user activity types that contribute 
to transition, and there are several more, such as peer finance, that are known to have featured in other transition process and are distinct from e.g. "userconsumers." Smith's recounting of the wide range of possible civil-society roles gives further impetus for asserting that it is simply too early to synthesize and close the list of relevant user roles, let alone user types, in transitions and move to testing a small set of them.

The construct of the phased relative importance of different user contributions may, in turn, be unverifiable. Evidence of some incidence of all eight types can be found in all phases in our study on heat pumps. But being able to show incidence is not evidence for the distinct phasing of importance. There would need to be a schema for empirically evaluating relative importance over varying incidences across the very different influencing mechanisms of innovation, adoption, consumption, intermediation, and legitimation. This is likely to remain impossible to do in a sound way, leaving the phased importance empirically unverifiable and thus very ill-fitting to the espoused "theory-testing" view of whole transition models. Theories meant to be tested must be testable, or else they are just rhetoric that rides under the disguise of academic rigor. ${ }^{3}$

Taking a step back from models on users in transition draws our attention to the status of high abstraction "overall story" transition models. To cover the considerable ground that in fact may remain between the overall story and the particular empirics in different countries, technologies, phases, and settings require mid-range registers and investigation programs. These need to be, on the one hand, detailed and sensitive enough to the studied phenomena and, for instance, refrain from imposing unverifiable constructs derived from high-level models (and need to rather question the adequacy such parts of the models), and on the other hand, they need to bridge sufficient amounts of empirical sites and times to arrive at grounded abstraction. This is the mid-range methodology road that the BOAP approach illustrates as being one possible avenue for future transitions research.

\section{Notes}

1 Statistics Finland, 2019; personal communication with expert Hanna-Liisa Kangas November 23, 2019, on how the "others" category in Finnish building statistics is divided in this regard.

2 Scott-Kemmis and Bell, 2010; Miettinen, 2003. This is particularly so as transitions models imply these processes are spread among a great diversity of actor groups and thus also dispersed data-wise.

3 In addition constructs such as "user-legitimators" who approve the new technology even if they do not adopt it (e.g., dwellers in district-heated blocks of flats that cannot switch to heat pumps but endorse them) can potentially cover everybody in the country. The question thus arises, how could one assess how the general population shifts its perception of an emerging technology? The methodological imagination coming closest to this would be a representative survey sent to a sample of citizens at set intervals, such as every three years, akin to political party voting polls, from the beginning of a transition to its end. The survey would, however, be seriously complicated by the fact that many of the 
sampled people would not be familiar with the new technology in the early transition phases, and hence the 2000 or so different people required to be contacted in Finland would need to be gauged not by simple mail or phone protocol but by qualitative interviewing supported by technology explanation. While this is a research design that is very unlikely to be ever be pursued, even such an exhaustive research design would still arrive at changes in the incidence of just one user role and thus still be unable to address the construct of relative importance. 


\section{Conclusions and implications for management and policy}

This concluding chapter spells out the overall importance of the analyses to the three theory traditions that motivate the book-namely innovation studies, transition studies, and S\&TS - and in doing so elaborates on citizen activities in long-term sociotechnical change. It further reflects on the application and development of the BOAP approach and draws implications for innovation, energy, and climate policies.

The book has linked theoretically and empirically informed sub-studies to a gradual grounded theorization, which can then be contrasted and compared to both sub-study findings and study framings, and onward to more generallevel models of sociotechnical change. We argue that this mid-range BOAP investigation strategy offers a better empirical and theoretical grounding to our view of users in sociotechnical change than has hitherto been available. Let us first examine what it offers for the intersection of innovation studies and S\&TS regarding S-RET innovation and community involvement and then move on to the intersection between S\&TS and transitions regarding user activities in sociotechnical change.

\subsection{The intersection of innovation studies and S\&TS: from local innovation and energy communities to hybrid Internet communities that aid wide diffusion}

Communities in their various forms are known to be key enablers and catalysts of citizen pursuits directed toward alternative technology. Innovation studies features an abundance of literature on innovation communities by users, as well as those hosted by companies (e.g., Jeppesen and Molin, 2003; von Hippel, 2005, 2016; Marchi et al. 2010), while S\&TS has had a more direct focus on energy technologies through research on technology-oriented grassroots innovation movements (e.g., Hess, 2016; Ornetzeder and Rohracher, 2013; Smith et al., 2016a) and community energy (Walker and Devine-Wright, 2008; Hargrieves et al., 2013; Smith et al., 2016b; Creamer et al., 2019). Most of this work examines communities as catalysts for innovation and anchors the discussion of communities to shared locality, or its digital surrogate, in which participants share a similar topical orientation, if not a joint mission, as in open-source projects.

DOI: $10.4324 / 9781003133919$ 
These innovation communities and citizen initiatives such as community energy have been found important in the initial invention and proliferation of alternative technologies. Be it solar collectors in Austria (Ornetzeder and Rohracher, 2006), wind turbines in Denmark (Karnøe and Garud, 2012; Nielsen, 2016), or mountain bikes, rodeo kayaks, and kite surfing equipment in their early venues (Lüthje, 2004; Lüthje et al., 2005; Hienert, 2006; von Hippel, 2005), it has been the early user innovators and their close peers that have generated a new type of alternative technology, initial practices of use, and new sets of meanings. These have then proliferated to other like-minded communities and often resulted in increasing adopter and community participant numbers, as well as further inventions (Ornetzeder and Rohracher, 2013; Hienert, 2006; Hienert et al., 2014). Furthermore, some of the alternative innovations have become mainstream through the commercialization of activities - a pattern found from innovations related to scientific instruments to the related hardware and software in enthusiast communities (von Hippel, 2005; Flichy, 2007; Williams et al., 2005) and in S-RET development as well (Ornetzeder and Rohracher, 2013). At the same time alternative routes to sociotechnical change have been proposed through the peer diffusion (von Hippel, 2016; Hyysalo and Usenyuk, 2015), mainstreaming, replication, and critical niche development of alternative technologies (Smith et al., 2016b; Kohtala, 2017).

Our analyses of S-RET user-innovation and user-run internet communities at once lend support to the above basic schemata regarding the early years of sociotechnical change. However, the analyses also show that the above view overlooks continued innovation by users in the later stages of sociotechnical change, the social value this creates, and the key differences there are between diverse and wide-reaching digital communities and local (or otherwise focused) innovation communities.

\subsubsection{Continued innovation by users in the later stages of sociotechnical change and the social value this creates}

That over 200 S-RET innovations were identified well after the early stages of the global and even local proliferation of the studied S-RETs poses the question of whether generally there may be this much "late" user innovation in S-RET and other already relatively stable and mature technologies. In Chapter 3 we identified that when a high diversity of user needs and settings prevails, areas of (gradually expanding) need space become too small for producers to notice or to yield required profits and are thus remain unserved by producers. This then results in continued unmet user needs that give rise to modifications and inventions by users (see Chapter 3, Figure 3.3).

Regarding the generalizability of this pattern, we can discern two analytical interpretations. The first interpretation is that the protracted blending of innovation and diffusion could simply now be more visible thanks to the traces the innovations currently leave in internet forums that would not have been there just a decade or two earlier to draw academic attention (Fleck, 1993; 
Hyysalo and Usenyuk, 2015). The second, and complementary, interpretation is that these hybrid internet forums in themselves catalyze some user innovation relatively late in the process of sociotechnical change through providing peer knowledge exchanges, peer encouragement, and a peer learning environment that pushes some users toward a deeper engagement with technology and toward adaptations, modifications, and innovation. In this interpretation, the unmet user needs would remain unmet if users did not get peer encouragement and support to meet their needs with their own solutions. Whichever the case the findings underscore the need to pay close attention to the "third generation of research" on user contributions arising from increased digital connectivity (outlined in the introduction) also in the energy sector.

The studied internet communities also underline a hitherto undiscussed form of social value generated by user innovators, namely in-depth knowledge production and sharing among greater peer networks. Our analysis suggests that in the presence of large-scale internet communities this may in fact produce greater social value than that resulting from the diffusion of user-made innovations. As discussed in Chapter 3, the direct diffusion of S-RET user innovation happened through manufacturers, users turning into entrepreneurs, and through peer adoption, but only in somewhat modest numbers. More user innovations were diffused by spurring on "innovative diffusion" resulting in further projects and adaptations to technology, which blends adoption and further inventive modifications. These projects and their sharing among peers build a technological peer culture among citizens, turn many user innovators user-side intermediaries, and aid market creation and legitimacy creation for the alternative new technology.

\subsubsection{The key differences between wide-reaching and diverse Innovation communities and local communities}

There are important differences between early local community forms and those that accompany wider proliferation and continued innovation by users. Unlike location-independent and diverse-orientation internet communities, the communities of deeply committed enthusiasts are not necessarily able to, or even likely to, grow into a substantial force in the later stages of sociotechnical change. Firstly, the locally anchored enthusiast communities face challenges in changing participant orientations to serve other users rather than advancing their own projects. Across community domains, the deeply knowledgeable community members have been found to soon tire of giving advice to evernew novices. Communities that feature greater diversity in orientations and activities also have a wider set of participant positions, which is conductive to providing the requisite help and to build a critical mass in membership (Freeman, 2007, 2015; Johnson et al., 2010). Secondly, in technologies such as S-RET supporting mass adoption is tied to the capacity to address the variety of settings in which the technology is used, the purposes it is used for, and the ways in which it is deployed-which all require mechanisms to effectively 
gain, transfer, and pool a knowledge base related to different locales, practices and preferences. It is not trivial for local enthusiast communities to create these, and if they do not, the problems encountered by diverse new adopters, adapters, and inventors will not be alleviated as peer responses simply remain too slow and too superficial to guide the help-seeking users in moving forwardwe also saw this in Chapter 4 (see also Murto et al., 2019a).

Concretizing the above regarding energy communities, community energy and internet-based new forms of energy communities provide differing and differently prospering channels to aid the proliferation of S-RET. Localitybound community energy initiatives premised on shared finance and shared produce (Devine-Wright and Walker, 2008; Creamer et al., 2018) are likely to face a steep barrier when aiding the wide spread of S-RETs: the local energy communities, even local grassroots innovation communities, are unlikely to have the requisite knowledge base and resources to help hundreds of thousands of others in their adoption- and development-related questions and concerns. Their capacity to do so could potentially be improved through pairing local community energy initiatives with internet discussion channels. But again the question remains about whether the typically ideologically motivated grassroots community participants have the wide variety of in-depth technical knowhow, as well as the motivation, to help differently motivated mainstream users and whether they would be willing to invest in the basic running costs, moderation, and site maintenance all this takes (cf. Smith et al., 2016b). The present book thus acknowledges the value of community energy as an important type of adopter setting and as a means to foster energy democracy locally, but it remains critical regarding the capacity and willingness of community energy initiatives to sufficiently support the wide proliferation of S-RET.

Concretizing the findings regarding user-innovation communities and diffusion support they may provide results in somewhat similar reasoning. Von Hippel has argued the case well for why user innovators do not invest in diffusing their innovations to the degree that their social value would justify unless they turn to user-entrepreneurs (von Hippel et al., 2017; De Jong et al., 2015). While we agree with the above reasoning, the framing should be expanded to ask what community forms may then be conducive to the diffusion of the technology alternatives produced by users?

Hybrid communities feature a mix of innovating and non-innovating users and exchanges about innovation, as well as just practicing, and in doing so they feature a wider variety of orientations. From technical sports-mountain biking, rodeo kayaking, moth sailing — we know that effective user-innovation diffusion happens among peers (Luthe et al., 2005; Hienert, 2006; Hyysalo, 2009; Hienert et al., 2014; Gambardella et al., 2017; Bethwaite, 2008). Common to these domains is the frequency of events and competitions, which creates spaces that are not focused on innovation per se but on practicing a sport or hobby (Hyysalo, 2009; Bethwaite, 2008). In such spaces, solution adoption happens through the imitation of winners - some of whom have invented or use the inventions by other users. Adoption also happens through discussions 
and advice given among peers on the latest developments, which includes user innovations and modifications, and is not limited to the discussions held among the relatively few user innovators. The key point is this: the hybrid community forms bridge several local communities (where more innovationdirected activities take place) and offer a setting where the benefits and makeup of all novelties are discussed as it is pivotal to the competent practice of the sport, and the latter process remains agnostic to whether they emerged from a producer or from a user. It is this wider diffusion-facilitating propensity that renders these hybrid forms of community effective in diffusing user innovation as well. ${ }^{1}$

Competitive high-tech equipment-reliant sports are of course particular domains. The diffusion propensity in a domain grows lower if the imperative to keep up with technical advances is less acute or if the diversity of needs is even greater, so that emulation of solutions by others does not yield similar benefits (Hyysalo and Usenyuk, 2015). The S-RET internet communities studied in this book exemplify a setting where these damping effects are in place-but which nonetheless allows otherwise geographically separated people to learn from each other-and a setting which is conducive for the general diffusion of S-RETs. As discussed in Chapter 3, the forums do not provide good support for the direct copying of the innovations made by peers and, consequently, feature somewhat limited direct adoption in a comparison of adaptive copying in DIY projects, again for both user- and producer-originated solutions. The diffusion of user-generated solutions could be facilitated by improved means for copying, a higher uniformity of needs, or more acute benefits from improvements to users. Regardless of the shortcomings of copying, the forums may be more powerful as user-innovation diffusion agents than a pure user-innovation community might be, for instance, the DIY section of a forum viewed in isolation or a more innovation-prone forum, which our interviewees noted as just lacking critical mass even for effective innovation support. It is further noteworthy that the capacities of communities to facilitate diffusion can, and often have, become curbed through moving the internet communities from open discussion platforms to "walled garden" settings, such as WhatsApp or Facebook groups, which do not support active moderation and archiving as effectively. Again, all the above underscores the importance of digital mediation in user contributions to innovation and diffusion and, equally, the very different patterns it can take depending on the domain characteristics.

To sum up the findings regarding user innovation and user communities in sociotechnical change, their impact is not limited to just providing technological solution variety at the onset of sociotechnical change that is then exploited or discarded by the market as a selection environment. Instead, this book has evidenced continued user innovation in tailoring the technology to new settings. Furthermore, the influence of user innovators has been found to reside beyond the particular solutions in the competence building and peerto-peer interactions, which are amplified through in-depth inventive engagement with technology and spread widely through the reach created by internet 
communities. Regarding the community form, the capacities of hybrid communities to aid both peer and overall technology diffusion may be superior to those of pure-bred user-innovation communities, firm-hosted communities, or locality-based energy communities. The peer governance, diversity of competencies, and plurality of orientations present in internet communities boost their capacity to provide timely and useful peer advice and thus to act as agents that can aid adoption, adaptation, and more deeply inventive projects.

\subsection{The intersection of transition studies and S\&TS: tracing the series of configurational movements to understand how users shape sociotechnical change}

The observation that active citizen users widely contribute to sociotechnical change beyond innovation and modification leads us to transition research that provides a framework in which the range of their contributions can be discussed and linked to each other.

In our analysis of Finnish heat pumps, adoption and a growing market are the prime mechanisms that signal user activities to other niche actors, as well as to the regime and landscape actors, and to potential users still aligned with the existing regime. Adoption is, however, importantly facilitated by a range of more active engagements with the niche technology. Adaptation and championing become relatively common when subsets of users face the need to alter the equipment or their social and technical contexts. As noted above, DIY and user-innovation activities add to the solution variety and signal further design needs to resellers and manufacturers and provide deep-level competence for intermediation in user communities, allowing these to build up into major repositories of knowledge and encouragement that aid the installations, troubleshooting, maintenance, and so on that are related to the novel technology. Our analysis further underscores that user communities are conducive to playing a role in market creation as they create and make accessible the qualifying and contextualizing information that potential adopters need in their purchasing and installation decisions. Wide user communities further aid in policing other actors in the market. The accumulation of internet discussion posts and their tendency to relate to the technology in a naturalizing fashion further contributes to building legitimacy for a novel technology.

In Chapter 5 we showed how these different activities by users feed into each other and note that many of these activities - particularly intermediation, market creation, and the building of legitimacy — became intensified with the expansion of internet communities. Without such publicly available open media, the peer capacity to affect not-yet users who are still aligned with the earlier regime would have existed to a lesser extent.

The transition framing is thus valuable in helping to extend the time frame in which to study how users drive sociotechnical change, as well as to expand the scope of relevant activities to be attended to in understanding how sociotechnical change becomes shaped (cf. Smith, 2012; Schot et al., 2016; Meelen 
et al., 2019). At the same time, conducting a long-term investigation into users that is also deeply informed by S\&TS and innovation studies draws attention to the need to build more accurate and fine-grained conceptual registers and models for transition phenomena.

Transition models have, to date, mostly operated at the level of the "overall story" of how particular sociotechnical transitions happen and different country contexts have been regarded as variants to the pathways formed within the transition (Geels et al., 2016). The same orientation is visible in studies of users and citizens during transitions. Findings on historical transitions in specific countries are abstracted in order to build models at the level of overall transition (see, e.g., Kanger and Schot, 2018; Kanger et al., 2018) and more in-depth studies on moments and sites of current sustainability transitions are equally treated as contributing to the overall transition models, whilst these deeper studies are almost exclusively from global early mover settings (see, e.g., Meelen et al., 2019; Truffer, 2003; Ornetzeder and Rohracher, 2013). These studies are then taken implicate that once the niche technologies mature to a point where the acceleration phase begins, they will then proliferate to societies in ever-widening numbers (Geels and Schot, 2007; Schot et al., 2016; Kanger and Schot, 2016), factually repeating the assumptions made in the (early) diffusion of innovation research (Rogers, 2010), with the addition that transition research stresses that the social-embedding process-changes in institutions, cultural image, consumer behavior, market models - is needed for the technologies to proliferate (Hoogma et al., 2002; Kanger et al., 2018).

But the unified overall story built on studies of globally early settings may not well represent the localized transitions where "rubber meets the road" if transitions to sustainability are ever to happen worldwide. The early-follower and later-mover settings interface with the alternative solutions that are already in a more developed phase technically regarding commercialization, distribution, and usage patterns. At the same time, the later-mover settings are likely to feature characteristics that differ from the overall story, as well as early development contexts regarding geographical conditions and the institutional environment (Truffer, 2003; Meelen, 2019; DeWald and Truffer, 2012; Meelen, Frenken et al., 2019), market conditions and differences in technology characteristics (Ornetzeder and Rochracher, 2013; Nielsen, 2016), local technology competition (Lovio et al., 2011), and issues of wealth and inequality (Smith et al., 2016); and dynamics among the users. Our analysis of S-RETs in Finland in the 2000s underscores how the overall story-level depictions can also miss out the emerging phenomena that later adopter settings may enjoy. A good example is the amplification that digital connectivity has introduced to the citizen's capacity to network, build communities, and to affect sociotechnical change.

The BOAP line of study subsequently suggests reconceptualizing users in sociotechnical change through two concepts: innofusion and a series of configurational movements. Innofusion means the development of a range of sociotechnical characteristics during diffusion, including significant improvement to the 
technology characteristics (Fleck, 1993; Heiskanen et al., 2014; Hyysalo and Usenyuk, 2015). The concept stresses protracted re-innovation in the product and system characteristics in response to varying and newly surfacing local requirements and opportunities. At the heart of this concept is the observation that technologies do not spread in a vacuum but face different environmental, market, institutional, and cultural conditions in new country, locality, and organizational arenas. Some smoother adoption and limited re-innovation of the technology tend to run in parallel in sites that are already better aligned with the new technology (Rogers, 2010; Mignon and Bergek, 2016). ${ }^{2}$ In this view, the zoomed-out view of a whole system transition (as an expanding technological niche and its social embedding) is simply not how the myriad of localized transitions play out, and at the end of the day, it is these localized transitions that comprise the overall change. New ways to depict transition processes are hence needed.

The innofusion process, in turn, features series of configurational movements-key biographic moments associated with shifts in the arenas and associated ecologies of actors - in which the material, social, and cultural characteristics of technology become structured and restructured (Hyysalo, 2010; Hyysalo et al., 2019a; Glaser et al., 2020). These movements typically start in more informal, fluid and rapidly changing configurations and gradually lead to a normalized or "cold" sociotechnical order where changes in technology characteristics, identities of users, and types of use, as well as the institutional environment, remain incremental (Pfaffenberger, 1992; Callon, 1991, 1998).

Tracing the series of configurational movements provides a lens through which to attend to the specifics of how new technology becomes socially and materially shaped in the course of sociotechnical change. Doing so helps identify the shifts and sites that have been consequential in the sociotechnical change, offering concrete points to look for in other localized transition processes and potentially supporting them. Examining heat-pump proliferation in the detached house market to about $80 \%$ of maximal market penetration in Finland - a country that was at first an early adopter but, after its initial market collapse, fell into an early-follower position-allowed us to draw out nine key configurational movements in Chapter 5 and to abstract a minimal set of configurational movements that occur in a successful follower country transition:

i. The discovery of underlying technology principles and design of basic solutions take place elsewhere; activities from both suppliers and users potentially feature.

ii. Initial domestic introduction and adoption result in a nascent ecology of actors, featuring activities from both suppliers and users and, potentially, various third parties.

iii. Adaptation, championing, and user innovation take place so as to better suit the technology to the consumer specificities in the country. These activities are primarily conducted by users. 
iv. The emergence of intermediation that lays ground for effective domestic market, which potentially features activities from suppliers, users, and third parties and can be dominated by any one of the actor group.

v. Further technology and market development elsewhere feed into the development of the domestic market (through, e.g., lowering prices, improved logistics, improved reliability, etc.), while the domestic market may also affect some of the technology development elsewhere, resulting in better-tailored systems for the particular market. This movement features activities from suppliers and potentially from third parties and users.

vi. The emergence of mass-market conditions and maturation of the industrial field in the country; activities are driven by suppliers, while users and Intermediaries play roles as well.

vii. There is an interplay with adjoining technologies and spin-outs to new sociotechnical configurations; users, suppliers, and third parties are all likely to be active.

We further note in Chapter 5 that some of these configurational movements are sequential $(1,2,3,6)$ whereas others may appear in parallel $(4,5,7)$, depending on biographic sub-trajectories, which may become reversed in the course of how the sociotechnical configurations and ecologies of actors develop. In Finnish heat pumps, for instance, we see a contingent "early curbing and death," "second introduction," "acceleration," "supporting intertwinements and maturation," and "spin-out biography" phasing.

Analyzing transition as a series of configurational movements calls into question some of the overall story assumptions, particularly those regarding the acceleration phase of transition. The abstracted set of configurational movements above and our later analyses of energy retrofits in housing companies (Murto et al., 2019a, 2019b) suggest that the smooth acceleration associated with the speeding up of diffusion and well-functioning markets for the new alternatives emerge quite late in the transition process. While the basic niche characteristics are built up during the take-off, the alternative novelties still proliferate against the grain of the market and institutional conditions set by earlier technologies for a considerable time. The acceleration phase might thus be split roughly in half, into a phase of "widening proliferation" and one of "diffusion through markets," which feature importantly different characteristics, policy responses, and managerial implications (see below for the policy and managerial implications).

Overall, this book argues that the study of users and citizens in transitions - similar to many other intricate transition processes that tend toward invisibility, such as learning ${ }^{3}$ - is not adequately addressed if only patched together from piecemeal case studies or from broad overviews that rest on limited empirical coverage. A recalibration of transition research is in order to address both the detailed processes and their long-term interconnections without losing the relevant details in a too zoomed-out view for the sake 
of gaining clear and easily graspable models (Farla et al., 2012; Zolfagharian et al., 2019; Murto et al., 2020). The BOAP framework is one candidate for the task of bridging the needed detailed ethnographic studies to the historical evolution.

\subsection{A user-domain focalized BOAP investigation: reflections}

The focus of the BOAP analysis in this book has been on user activities in the course of a long-haul sociotechnical transformation. The S-RET systems studied present an "industrial field of medium complexity," It is, for instance, considerably less complex and features far fewer arenas and smaller ecologies of actors than the organizational software studied earlier (e.g., Pollock and Williams, 2008, 2016). At the same time, it is considerably more complex than most consumer good fields (e.g., Baldwin et al., 2006) as S-RET systems involve some permitting and other institutional regulation, require separate installers, are relatively costly, and have to integrate into very diverse housing settings and everyday practices.

The study has been purposively cast to focus on one country and one group of technologies, with an added weight on heat pumps that, thus far, are the most widely spread of the S-RETs in Finland. The data gathering has been a mix of ethnographic and historical analysis typical of BOAP, and similarly a mix of several more detailed foci, wider overview analyses, and quantitative and qualitative analysis. The focus on sociotechnical change in one country context and user activities has meant that wider "global" changes were examined as an endogenized context, that is, it was included insofar as these developments have had a bearing on the national and local developments. The same goes for various neighboring developments to the S-RETs in Finland during the extended study time of about 30 years - they have been covered from other research and secondary literature and then linked to the focal analyses insofar as they are relevant. The approach presents a new way to contextualize user activities as well as to focalize long-term sociotechnical change research onto mid-range phenomena.

The present book features a one-side biased biography of a sociotechnical change in that the arenas and ecologies of actors beyond Finnish user activities have been studied more thinly. In particular, the international context where most S-RET producers have become located in the Far East has not been studied first-hand. These limitations do not render the approach meek. BOAP has guided the researchers into attempting to trace the endogenized influences back to a national context and users, and guarded the analysts and readers from reliance on single snapshots or hollow-arch overviews, as well as from pretending that the present account tells the whole story. BOAP also points out the possibilities for further research by extending the study to the now implicated international arenas, as well as in continuing the Finnish context research through tracing the future trajectories of the renewable energy technologies, particularly because their installation types and scales are presently expanding 
from residential systems to a larger community and industrial-scale deployments, which at once shifts both the relevant arenas, the ecology of actors populating them, and the sociotechnical configurations involved.

The present book further elaborates on the dual orientation that characterizes most BOAP research: on the one hand, as (just) a methodological guideline and, on the other hand, a methodology that becomes (re)connected to a more encompassing theory tradition.

In the present book the status of BOAP was first that of a methodological approach to building and extending the research design. In this capacity and long into the accumulation of the empirical work, the findings would have been compatible with several different theory frames. BOAP as a methodological approach tied the analysis to the study of emergence, the more durable structures in place, and their transformations, and thus forced the analysis beyond a bird's-eye overview of systems change, a focus on particular topics (such as user innovations), or making a snapshot study of actor networks in one locale related to S-RETs. The additional merit of using BOAP as "just" a methodological guide is its capacity to steer towards theory bridging and interrogation, which was suggested as the ninth typical feature of BOAP studies in Chapter 2. The stringing of the sub-studies typical to BOAP leads analysts to relate to the substantive research traditions and their research designs that are relevant to the sub-study phenomena. In the present book the relationship to these theories has been appreciative, yet their juxtaposition and empirical insights have qualified each theory tradition with respect to what they cover and what they leave unattended (see the above summary findings). In this capacity, the BOAP methodology has a propensity to create a double epistemic inquiry: one related to the research domains and another related to the theories, study framings, and research designs that have been used to study the research domain.

A second orientation to BOAP emerged in the later years of the decadelong study on user activities in S-RETs in Finland. The status of BOAP moved into one that was (re)connected to substantive theory tradition in ecological sociology once it became important to better understand the ecology of actors, and user contributions to intermediation and market creation. Similarly to earlier work on health care technologies (Hyysalo, 2010), where the study was explicitly informed by activity theory and symbolic interactionist S\&TS, also here the symbolic interactionist social worlds/arenas framework guided how the activities of peer communities and ecologies of intermediaries were linked to ecologies of actors, the emergence and stabilization of arenas, and endogenized causation to arenas elsewhere. This (re)connection to substantive theory helped both the theoretical sampling in the final years of the BOAP investigation and in giving a final interpretation of the results.

It is important to notice these two orientations to BOAP could also run on colliding courses. If the connection to any one theory tradition was strong from the outset, an appreciative theory bridging BOAP sub-study framings 
would be unlikely to happen, and instead a single competing theoretical account would emerge. Conversely, if the BOAP was only used strictly as a methodological guide and theory only informed the final interpretation of the gathered results, it is likely that the methodology and methods will not have sufficiently informed the data gathering so that the issues important to that theory would be gathered. In the present book middle ground was sought between the merely methodological and theory-connected use of BOAP.

Regarding generalizing from a BOAP investigation, a careful reader might have noticed that there are two implied avenues for generalization in the present study. First, the sub-studies on user innovation, on user activities in the energy transition, and on the nature of technology-oriented peer communities link to wider bodies of research on the same topics, and results from Finland (which is in many respects a typical Western industrialized country that has identifiable contextual and energy economics features that help comparing findings across countries; see the Appendix) and its S-RET-related phenomena add to the stock of findings that offer generalizability in these bodies of research.

Second, beyond the sub-studies, the investigation pursued through the BOAP methodology offers a generalization on sociotechnical change dynamics that is premised on deep and extended lines of enquiry, which traverses between highly situational snapshot studies and a broad overview and system change accounts, which tend to rest on hollow-arch empirics. Metaphorically speaking, it provides construction beams that aid linking more short-term or more superficial studies to one another or, to use the metaphor of quilting, it provides long pieces of canvas and long fibers that give strength and structure so that shorter pieces can be added in. The net importance of the latter generalization strategy is that BOAP-type investigations can provide needed empirical grounding to "middle-ground theory" building of sociotechnical change phenomena, particularly on topics that tend to remain invisible in official statistics and other record keeping that require empirical primary studies.

\subsection{Facilitating user-driven energy transitions: managerial and policy implications}

\subsubsection{User-driven energy and energy technology business}

Energy systems have traditionally presented one of the most centralized and supply-side oriented environments to the extent that consumers are commonly referred to as "the demand side" and supplier offerings of power and heat feature remarkably small variations. Climate change and the ensuing imperative to transition to fossil-fuel-free-energy systems are rapidly changing all this. It is widely recognized that energy companies need to change their business logics from being the carbon problem to resolving the carbon problem. Doing so implicates that companies in the energy sector must also become customercentered and engage in user-driven transformation as consumers require new 
services and options. In turn, climate and energy policy should ensure that this is properly incentivized and sanctioned. But what exactly does this user-driven transformation entail?

The analysis in this book suggests that most of today's customer-centered actions by energy companies are still best characterized as seeking to develop solutions for potential customers, including:

- increasing the diversity of services from just two to three pricing options to options for different energy sources and mixes;

- introducing energy plans with two-way sales for prosumers;

- introducing options to be part of demand-response measures through virtual power plants;

- introducing options for renting or leasing solar panels or other renewable production;

- providing more sophisticated energy monitoring devices and interfaces;

- providing more sophisticated building automation (and monitoring) services to reduce energy consumption;

- featuring evaluation services to assess where energy efficiency could be improved in the house;

- energy-use reduction as a service (ESCO), where energy efficiency measures are billed from the differential of reduced heating bills over time;

- building area-wide solutions for heat/cooling and power storage to handle intermittent production and fluctuating prices, and to provide better services for customers.

All these are highly necessary for energy transition. But from a user-driven innovation and business perspective, these are not far going or very transformative renewals. In fact, such measures represent the energy sector as barely on a par with business-as-usual in most other industrial sectors. Being "user-driven" boils down to added market segmentation and service differentiation to offerings, and using service design to ensure the new services and products feature sufficient user experience (Hyysalo et al., 2016a; 2016c).

Our analysis of S-RET systems in Finland shows that once consumers gain the possibility to choose and operate their own energy systems, they grow more active, just as they have done in other domains such as ICT. This implies that energy technology providers and energy companies can move towards a deeper user-driven orientation in their strategies and operations, one that entails not only designing for customers but also designing with customers and engaging with the development done $b y$ customers (Prahalad and Ramasvamy, 2004; von Hippel, 2005; Hyysalo et al., 2016). Such a portfolio would minimally entail business offerings such as:

- providing a platform for and offering a pooling service for establishing energy communities between neighboring residential buildings, which would provide active energy citizens with more flexibility in balancing 
their prosumption profiles and would help achieve larger community energy systems; the energy company would gain commission and potentially the energy contract from the community;

- venturing into energy retrofit business to build deep retrofits and financing them as a "one-stop shop" with the energy service company business model;

- $\quad$ acting as a broker to pool willing houses or housing companies to form an energy community and to achieve network effects in deep retrofitting, effectively supporting the retrofit venturing;

- hosting or supporting internet communities in emerging areas, such as deep retrofits, hybrid heating systems, and energy communities; the benefits would accrue from learning about the market and helping it grow, similarly to what has happened to the residential S-RETS studied in this book.

As careful readers recognize, the above suggested 'with' measures necessarily feature business partnerships and the diversification of business for energy and energy technology companies, particularly into energy retrofit markets. An underlying condition is the acceptance of the ambidexter business model, where traditional energy companies accept volume loss in their traditional heat and power sales to existing customers and compensate for it with new business that brings rents from building higher energy efficiency and from the attraction of new customers. In this line of thinking, the energy company or energy technology company is foremost out to serve its customers' active or potential initiatives in achieving a lower and more renewable energy production profile. The financial benefits follow from being a pioneer in pursuing deep customer orientation and gaining brand recognition for advancing the green transition. There is thus a considerable difference to a "for" orientation, where these companies still intend to push for more sales of their products even if through more diverse and additional services-inclusive business model.

The rationale for underscoring the deep user-driven transformation is that S-RET installations, deep retrofits, hybrid systems, and pooled energy communities are still in their globally nascent stages and the difficulties in acquiring them curb most customers from taking action (Murto et al., 2019a, 2019b). And, similarly to residential S-RET in the Finnish context two decades before, there is a strong latent demand premised on environmental benefits and positive cost-effectiveness. Such conditions arguably lead to these markets becoming formed at some point-either through entrants, via more piecemeal solutions, and/or through the strategic actions taken by some forerunner incumbent companies.

Moving yet deeper into actions taken by consumers in transitions, our results underscore three managerial implications. First, energy and energy technology companies could shift to open user innovation orientation in gauging and seeking to help those citizens and citizen groups that try to create new sustainable energy solutions. Not all business and innovation wisdom recides within 
the companies and being involved in experiments lead by citizens offers first mover opportunities to learn and develop further both technical and business solutions. Second and related, monitoring user modifications and innovations may reveal points for innovation for technology manufacturers. User alterations reveal both need and solution information and can be analyzed regarding both (von Hippel and von Grogh, 2016). The information on needs was previously difficult to highlight, but with internet communities, user problems, wishes, alterations, and innovations it is now readily available for producers to follow (Grapher and Ibert, 2013; Johnson et al., 2010; Hyysalo et al., 2015). Regarding solution information, many user innovators are happy to freely reveal their solutions, and companies could also volunteer to further develop the best DIY ideas with a fair compensation scheme in place-the sums users ask for are commonly low if they even ask for a sum (von Hippel, 2005, 2016). Our results indicate that it may make the best sense to monitor where the solutions are clustered and what kind of adoptions and innovation pathways they form together as this articulates demand and points to improved commercial systems, thus helping to move beyond the problems of isolated solutions to very particular user needs (see Figure 3.3; Nielsen et al., 2016). Thus, on entry to emerging energy technology markets, producers would benefit from following the interaction arenas where users discuss the technology and display their problems and solutions.

Third, companies or industry associations may wish to sponsor user-run internet communities that feature effective archiving and moderation. Even as some of their actions, such as policing the field against poor installations, may be irritating in the short haul, there is a clear market organizing and legitimacy-creating effect over the long haul. These effects will not, however, come to fruition only through Facebook groups or other non-moderated and ineffectively archived social media. Without moderation, an internet community can quickly grow useless for all concerned through, for instance, false claims, tarnishing campaigns, and libel content. Without archiving, prospective buyers and users will not be able to effectively find the information they need.

\subsubsection{User-driven energy and climate policy}

For policy action, the core finding of the analyses in this book is that it takes a long time before effective market and informational mechanisms accompany supplier and user solutions in such a way that there is a well-functioning market for new energy technology. The cumulative sales figures can run into tens of thousands of units, yet the ecology of actors may still remain ineffective in pushing low-carbon solutions for true mass adoption. The common policy reaction is to focus action on the supply side, and to provide relatively minor and undifferentiated subsidies to the demand side. This is commonly paired with only gradually changing regulation and other institutions in order 
to better support the new alternatives, typically through processes slowed by lobbying by incumbent businesses.

Our analyses lend support to the view that new alternative more sustainable solutions face obstacles and hindrances due to having to operate in a market and institutional environment built for the technologies they would replace (e.g., Hoogma et al., 2002; Murto et al., 2018a; de Rubens et al., 2018). Various hindrances in, for example, permissions, regulations pertaining to pricing, thresholds set for subsidies, taxation, etc., may continue well into the proliferation process (Heiskanen et al., 2014; Berninger et al., 2017). When these are paired with the information and productization deficiencies that are common to the early phases of a new technology type and with missing market actors and market information, the summative damping effect imposed on adopters can be considerable. Environmental policy should thus be more deeply intertwined with innovation and technology policies (see below) and take the need to monitor the formation of effective markets for more sustainable alternatives seriously - and be ready to support it. The support measures can happen through removing unnecessary or poorly motivated market hindrances as well as through setting up experiments in which more level playing field is provided as well as through public or public-private intermediary actors to support the emerging niche markets once they have shown their potential yield to environmental benefits.

In addition to supply-side and market-structuring measures, the present analyses underscore that users are more active across the long process of sociotechnical change than the mainstream policy advice notices, and their capacity to contribute to advancing sustainable sociotechnical change can be supported beyond incentivizing adoption.

First, recognizing citizens as active and well-informed actors calls for a rescaling of climate and energy policy from the present top-down one to one that actively responds to and facilitates initiatives on the ground (Jalas et al., 2017). Finland alone features several citizen-initiated climate actions along with internet forums that directly evidence need and enthusiasm. These feature a carbon-neutral municipality network in which presently over $30 \%$ of the country's population has joined and, at a much finer level of engagement, housing company clubs that pool housing companies for energy retrofits. In both cases, climate policy actors have joined and come to facilitate and provide longevity to the initiatives. The main locus of action in responding to such measures is likely to reside in municipalities and areas, but also national bodies In Finland energy efficiency agency Motiva, have shown themselves capable of recognizing and responding to such initiatives. Providing funding for running and expanding such citizen initiatives and linking them with technology experiments and legislative trials are strategies that are already pursued in many countries and clearly should be pursued even more in the future.

Second, under the conditions of sector-wide transformation, energy, innovation and environmental policies should be pursued in tandem (Hyysalo et al., 2019b). The policies should be further linked to cross-sectoral measures 
to remove the typical mesh of existing regulation that unnecessarily blocks or hinders citizen choice and engagement with new types of technologies ranging from specific items related to permitting, taxation, ownership rules, actor responsibilities, and so on that have been formed under the conditions of the previous technology base. This calls for the active monitoring of changing sociotechnical conditions and typically calls for active engagement with both citizen communities, and intermediaries and researchers who work with the cutting-edge change. Such action can be rendered anticipatory through multi-stakeholder arena processes (Hyysalo et al., 2019a, 2019b; Lukkarinen et al., 2020).

Third, peer support among consumers is a valuable resource that could be cultivated. Peer support can foster deepening engagement with energy technology that leads to higher consciousness of how much energy is consumed and how it has been produced. With regard to facilitating internet communities themselves, it may be illusory to seek to create such discussion forums through policy measures, but allowing (often anonymous) discussions to prevail holds benefits, and some energy and climate policy actors, such as energy councilors, might offer their advice through these discussion forums, as has already happened in youth work after the realization that nowadays it is increasingly on the internet where people can be reached (Hyysalo and Juntunen, 2018). Some forums may benefit from nominal support in order to cover running costs (see the above regarding corporate sponsorship).

Importantly, peer-to-peer advice, local energy communities and internet communities should be regarded as important and thus be explicitly assessed when passing regulation. For instance, measures to counter libel content on the internet by removing all anonymity could also wipe out positive anonymity in internet discussions, whereas displayed anonymity that is traceable to real verified persons in cases of libel action would not. Also, the nature of commercial internet plans available to consumers should be monitored: open internet should be retained as a possibility in open society to avoid lapsing into the availability of only "walled garden" ecosystems by leading commercial providers as this can severely limit the effective forms of digital citizenship available. These arguments are part of innovation wetlands-thinking (Torrance and Von Hippel, 2016) in that diversity and exploration by citizens is a valuable but unflagged resource that is easily erased, similarly to how seemingly useless wetlands have come to be recognized as hotbeds for biodiversity and their erasure requires consideration. Needless to say, the same goes for consumer innovations and DIY activities.

Regarding user innovations, policymakers commonly view them as too isolated and particular for targeting policy action (Nielsen et al., 2016). As indicated above with managerial measures policy action could target areas where clusters of user innovations are occurring and build programs to foster producer-user interactions in order to develop the user improvements into commercialized offerings. 
This said, we should reflect on the limits to policy action such as the limits to what policy measures can do to counter the potential under-diffusion of consumer innovations in S-RETs. As argued in Chapter 3 innovating consumers would have the incentive to take the time and energy to document their often complex hacks and designs for others to easily adopt them. The innovating consumers could, however, be motivated to do so if there were effective platforms and templates available for the effective display of their problems in such a way that it would help to present them and, as a side effect, make a more structured information package out of the display (e.g., if an easy way to make blueprints was available or if there was an easy way for the user to point to the exact location of the modification and make parts lists). But what could policy provide? Not much apart from funding a research program or potential challenge competition aimed to create better sharing platforms for physical citizen designs.

Throughout the course of this book we have shown how activities by citizens can turn many core assumptions about energy system change upside down. Ordinary people innovate, they structure markets and, gradually, their purchases can drive a wholesale change regarding heating practices and, by implication, can also power their own lives with an S-RET that generates electricity. These processes may benefit from policy intervention, but foremost they may only require the absence of policy blocking. Similarly, technology development and mass-manufacturing economies are required to get the technology options to the point of rough cost-effectiveness, but again these must not all be perfect before the innovation-in-use kicks in and brings in further improvements. Incumbent energy companies and energy technology providers may either join or seek to initially curb the process but would be fools to think they would have the power to stop citizens from wanting and driving the transformation of energy systems in the long haul and across country contexts.

\section{Notes}

1 Needless to say, the exact dynamics would merit careful studies of the diffusion and recommendation behaviors in such communities.

2 Innofusion is likely to be more common with S-RETs that are deployed in wide and variable ways, at different scales, and with different ownership models (such as is the case with heat pumps, energy efficiency renovations, or pellet and solid-wood burning) than in S-RETs that require permitted and uniform installations, such as community-scale wind power (Juntunen and Hyysalo, 2015).

3 See Chapter 1 and Chapter 5: These processes are invisible because they are not recognized by the actors experiencing them as occurring or being of importance; they are consequentially not systematically recorded and do not reliably show in easily accessible data series or in using standard research instruments. Any one of these conditions being present leads to a requirement for considerably more arduous research designs than is customary in mainstream transitions research. 


\section{Appendix 1}

\section{Data and methods on renewables innovation and adoption in the Finnish energy system}

\section{A.1 The Finnish energy system and S-RET adoption and innovation}

Similarly to energy sectors in many European countries, the energy sector in Finland has rested on centralized large production units and its energy policy has historically concentrated on the security of supply and affordable energy prices, particularly for industry. International agreements and EU directives on climate change mitigation have contributed to a gradual sector-wide transition toward lower emissions in this millennium. With respect to the overall share of renewables, Finland features the second-highest national share of renewables (42\% in 2018) in the EU-27, largely owing to its forest industry's by-product energy use, other combustion of forest biomass, and hydro. The carbon emission reduction has also been helped by prolonged economic austerity since 2008, resulting in a decline in both energy consumption and $\mathrm{CO}_{2}$ emissions (Statistics Finland, 2019), making Finland one of the few European countries to reach its 2020 and 2030 targets in advance. The structural changes toward increasing low-carbon production and distributed energy production's share in the Finnish energy system have, however, been relatively slow in a Scandinavian comparison. The energy sector transformation is likely to speed up in the near future with the present government aiming to halve petroleum use by 2035 and phase out coal use by 2030 and peat use by 2035 (The Finnish Government, Marin Government program, 2019).

Examined in greater detail, the overall energy consumption in Finland grew until 2006 and peaked at $1500 \mathrm{PJ}$, after which it has declined to $1300 \mathrm{PJ}$, roughly at the level of the year 2000. Per capita, Finnish energy consumption and $\mathrm{CO}_{2}$ emissions (10.7 tons of $\mathrm{CO}_{2}$ in the year 2020; Eurostat, 2020a) are above average in EU owing to the country's northern location with an annual average temperature of $2^{\circ} \mathrm{C}$ associated with high seasonal variation in both the temperature and availability of sunlight. Electricity prices in Finland have traditionally been among the lowest in Western Europe (EU-15). In 2018, industry consumed $46 \%$ of total energy, transport $17 \%$, and the heating of buildings $26 \%$. Finnish buildings are well insulated but, 
as is typical of northern countries, people are accustomed to high and stable indoor temperatures, the average being $21^{\circ} \mathrm{C}$ (Heiskanen et al., 2019).

Finland's energy mix in 2018 featured wood (27.2\% of all energy), oil $(22.4 \%)$, nuclear $(17.3 \%)$, coal $(8.2 \%)$, gas $(5 \%)$, hydro and wind $(5 \%)$, peat (4.5\%), and other sources (4.8\%), and a net electricity import of 5.2\% (Statistics Finland, 2018). From electricity production in 2019, nuclear power accounted for $26.6 \%$, hydropower $14.3 \%$, wind power $7 \%$, while fossil fuels had an over $20 \%$ share through the widespread use of combined heat and power production in district heating. Net imports covered 23\% (Statistics Finland, 2019). Intermittent renewables shares have grown rapidly in the latter half of the 2010 s, but solar still accounts only less than 1\% of electricity production.

The energy policy in Finland has remained in the hands of a cohesive and narrow technocratic and business elite (Ruostetsaari, 2010). The supply has concentrated on privately owned companies, municipal energy companies, and the state-owned Imatran voima (Heiskanen et al., 2018). These actors created Finnish Energy, an industries association, in 2004. On the demand side, large energy-intensive businesses have been central players. The energy division of the Ministry of Economic Affairs and Employment has become the central coordinating body that listens carefully to these supply- and demandside actors while the state's technical research center has held a position in providing the scenario modeling tools used (Heiskanen et al., 2018). Heiskanen et al. (2018: 62) summarized that, in this elite, "[m]embers come from the same schools, share the same ideals, and throughout history, have created common field-level rules, which emphasize security of supply, affordable energy, an engineering ethic and the importance of economies of scale" (Ruostetsaari, 2010; 2020). Energy policy has remained a relatively depoliticized issue among the parliamentary parties. The exceptions have been the NGO and citizen campaigns against new nuclear and hydropower plants, which have stopped some of the latter but did not alter the decisions for fifth and sixth nuclear plants and a permanent nuclear fuel repository being given permits in crossparty votes in the 2000s. Importantly, the "against" campaigns have not been associated with a strong "pro-renewables" movement, such as that in Denmark where the emergence of wind power experiments and the later surge for wind formed a central aspect of anti-nuclear movement (Ornetzeder and Rohracher, 2013; Nielsen, 2016).

The Finnish energy policy has been slow to impose structural changes toward more distributed and intermittent energy production (Ruostetsaari, 2020). A feed-in tariff for wind and some biomass was introduced in 2011, late in European comparison and exclusive of smaller units with less than 100 $\mathrm{kVa}$ that were deemed as administratively impractical (Heiskanen et al., 2018). Finland also remains the only EU country that never introduced any dedicated support for solar energy. The slow uptake of structural changes has been legitimated by the second-highest national share of renewables in the EU-27, even though it rests heavily on the forest industry's by-product energy use, which is likely to reach a limit through land-use regarding carbon capture. This said, 


\section{Appendix 1}

Finland was a pioneer in smart meter roll-out, completing it in the first years of the 2010s, and has since pioneered demand-side solutions, such as virtual power plants and remote automated energy solutions for offices as well as homes.

In the latter half of the 2010 s, energy transition began to move into the next gear due to the tightening of international agreements and EU carbon trading, as well as the intermittent renewables and large-scale heat pumps that were becoming cost-competitive and thus more legitimate in the Finnish energy policy discourse. The 2019 government change meant new targets for achieving a net-carbon-neutral society by 2035 , which has resulted in the endorsement of structural renewal and investments in both political and corporate discourses, albeit with a stress on cost-effectiveness (The Finnish Government, Marin Government program, 2019; Finnish Energy, 2020). Also, measures to improve the energy efficiency of the housing stock beyond the existing, internationally high insulation levels has gained new impetus in policy during the 2010s (Heiskanen et al., 2018, 2019). Most recently, energy prosumption and energy communities have become recognized as part and parcel of reducing the emissions from the housing stock (Pahkala et al., 2018; Lukkarinen et al., 2020). In these developments S-RETs, particularly heat pumps, have become to be highlighted as a viable example.

Over the years, the adoption of small-scale renewables (S-RETs) has only been modestly advanced by subsidies that have addressed energy efficiency and renewable energy use in the residential sector in Finland, such as subsidies for replacing oil-based heating systems at the end of the 2000s and again in 2020 . The modesty is evident in that the most important S-RET subsidy has been the household-duty deductible, a maximum of $€ 4 \mathrm{k}$ annually per household, aimed for buying services such as cleaning and repair but appropriated by many to include a portion of S-RET installation costs. Regarding energy use in housing, Chapter 5 provides an overview.

Energy prices relative to S-RETs for households are an important factor in explaining the market-driven energy transition in Finnish detached housing from fossil-based sources toward renewables. An comparison by the European Heat Pump Association (EHPA, 2018) made a strong argument regarding the importance of the relative price of gas and light fuel oil to electricity and, onwards, to pellet and district heat prices as an explanation for the uptake of heat pumps and other S-RETs. In the EHPA's calculation, countries where heat pumps have been adopted widely all feature a situation where heat-pump technology and the household electricity used by heat pumps is cheaper than any other heating source. The European countries that have favorable energy prices are Finland, Sweden, Norway, Austria, the Czech Republic, Latvia, the Netherlands, France, and Portugal, as well as Estonia as a borderline case, regarding relative gas price. Out of these the three Scandinavian countries all feature high heat-pump uptake, while the uptake in the Netherlands remains low. The converse price implication is that solar PV is relatively less profitable in these countries than in countries where electricity is expensive. 
The other end of relative prices features countries where gas and some other heating forms are cheaper than electricity and heat-pump energy. These are Germany, the UK, Belgium, Italy, and Denmark, in which heat pumps continue to face adverse profitability compared with fossil fuel options, and in none of which heat pumps have proliferated widely. Again correspondingly the incentives for producing electricity with PV are higher and indeed PV uptake is high in most of the countries in this group. In between lie countries where some of the energy alternatives are roughly on a par with heat pumps. These are Ireland, Hungary, Spain, and Poland, with Slovakia as a borderline case regarding relative gas price. The implication from the relative energy prices is that, for our analysis in Chapter 5, comparisons between each country group could be informative, for instance, comparison between high heatpump proliferation countries (NO, SE, FI) against NL where heat pumps have not diffused despite similar relative energy price ratios. Similarly, comparisons of transition dynamics between Finland and in Denmark, Italy, Belgium, and the UK would make less sense beyond noting that the energy economics create very different dynamics.

The housing stock and ownership structures in Finland are well-suited for the installation of S-RETs. Ownership of homes in Finland is 71.5\% (Statistics Finland, 2019), which is higher than in most of Western Europe and above the EU-27 average of 69.2\% in 2016 (Eurostat, 2019a). Detached houses are home to $48 \%$ of the population, and an additional 13\% live in row houses (Statistics Finland, 2019). A large portion of consumers thus have direct or easy access to making decisions about and even modifying their heating equipment since there is nothing legally banning them from doing so.

The Finnish population is at the top of the UN education index and the country has retained a do-it-yourself culture, particularly in the sparsely populated countryside, which both contribute to having the skills to build and tinker with equipment, as well as to the capacity to search for and appropriate information. Finland is reputed to have had a tradition of peer help owing to its relatively recent agrarian past and wartime efforts, but these traditions have withered with the rising of standards of living, particularly since the 1980s. All these facets are likely to support Finns' capacity and willingness to adopt, adapt, invent, and set up extensive communication forums on how to handle technical novelties.

At the same time, Finland only has a few small manufacturers of heat-pump and pellet-burning technologies and there are virtually no supplier ecologies that could spur on user inventiveness. As noted above, there is also relatively little renewable energy activism that could spur on user inventiveness or other active forms of technology engagement in S-RET (cf. Ornetzeder and Rohracher, 2006, 2013). Summing up, while the economic incentives toward S-RET adoption, DIY projects, and invention may be somewhat lower in Finland than in most developed countries and in many middle-income and developing countries, the Finns' capacity to modify and do things with technology may be higher or at least formed differently. 
Regarding peer support over the internet, Finland has a large number of internet users and it forms a specific language area with less than 6 million people, which may contribute to citizens' willingness to engage in specific online communication forums. The anonymous public display of opinions, advice, and modifications does not allow the identification of who exactly made, for example, a potentially hazardous hack that could compromise house insurance (cf. Torrance and Von Hippel, 2016), arguably contributing to free and wider sharing of information among peers.

The first S-RETs internet discussion forum for GSHPs emerged in 2004 (www.maalampofoorumi.fi) and the second, for all heat pumps, in 2006 (www .lampopumput.info). These were followed by a forum for wood burning systems in 2007 (www.pellettikeskustelu.net) and for "free energy" (www.ilmaisenergia.info) in 2009, in which micro-wind power, solar heat, and solar PV became clustered. Over the years these have evolved into a major communication medium among the citizen users of these energy technologies. By the end of 2020, the largest of the heat-pump forums alone (www.lampopumput .info) had been viewed over 200 million times in its 15 years of existence. These forums are not Facebook groups or general online message boards such as those found in AOL or Suomi24 (Finland24) but moderated, categorized, and accumulative internet communities. Such forums exist worldwide for software and hardware technical support and for consumer exchanges on a variety of goods, such as bicycles or cars. The discussions are typically anchored to specific problems with specific technologies: their features, makes, and models, as well as some typical usages and activities associated with those technologies. Such forums proliferated rapidly after 2001, when easy-to-use forum software platforms became available for everyone. Heat-pump-related internet forums in many other countries are of similar magnitudes. For comparison, Sweden's Varmepumpsforum focuses solely on heat pumps and was established in 2004; it has over 40,000 registered users and had had over 660,000 messages posted on it by 2016. In Germany, the largest forum (www.haustechnikdialog.de/ Forum/30/Waermepumpen) has attracted active discussion with over 20,000 topics (i.e., it is of the same size as the largest Finnish forum). In Norway, the discussion has been scattered around different internet sites. Some of the sites include thousands of topics concerning different heat-pump technologies (e.g., http://byggebolig.no). In North America, several home improvement, repair, and DIY websites cater for heat-pump discussions with a rather limited number of message threads.

\section{A.2 Data collection and analysis}

\section{A.2.1 User innovation and the diffusion of user innovations}

To investigate innovation by consumers and its diffusion, our research formed a sequential mixed-method study. Figure A.1 provides an overview of the research process. To identify consumer innovations, we used a combination 


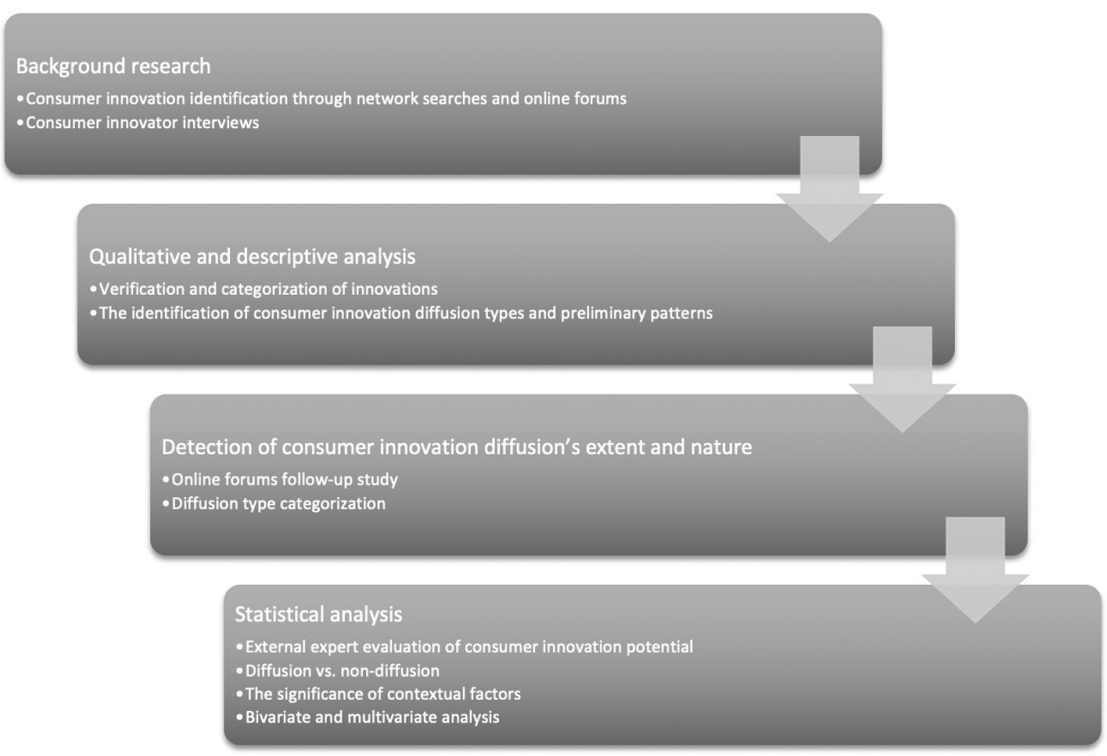

Figure A.1 The sequential mixed-method research design used to study user innovations.

of network search lead-user identification methods (Hyysalo et al., 2015). The searches came to center around Finnish online internet discussion forums on renewable energy (www.lampopumput.info, www.maalampofoorumi.fi, www.pellettikeskustelu.net, and www.ilmaismaisenergia.fi) as most of the found consumer innovations were displayed in these. We sampled and stored typical postings in all of the forum sections that we suspected could reveal inventions, most notably the "modifications and improvements" and technical questions sections (from here on, "DIY sections"), which, for instance, for ASHPs, featured 1206 discussion threads. We read through all these threads in their entirety and coded them. To gain a better idea of the inventions and discussants, and their relationship to those in the DIY sections, we used the general statistics of the forum, as well as manually checking the full user profiles of 115 discussants active in DIY sections. We then conducted 30-120-minutelong semi-structured interviews with 47 forum-active inventing consumers and five (5) firms that had collaborated with inventive consumers, focusing on the modifications that these consumers had made and their information sharing with other consumers and manufacturers. In identifying the user innovations, we used multiple sampling logic and a search-string technique we call a mountaineering search (Hyysalo et al., 2015).

Our data analysis proceeded through content analysis and categorization of all inventions and modifications. We then categorized findings regarding technology, an improved subsystem, and the achieved gain (efficiency, suitability, 
maintenance, or cost saving). In total, we identified over 400 potential DIY projects that could have been consumer innovations and out of which next to 300 projects were such that our scrutiny as non-domain experts indicated that these could be both

a) realized and working, creating a benefit for their maker and not just hacks that were not completed or were unsuccessful;

b) innovative, either in terms of novel functions or the novel material realization of a previously identified function; if the consumer project was relevant to a previously known function, the material realization had to be novel and it had to have a clearly identifiable benefit.

Figure A.2 presents the formal criteria for deciding which projects were consumer innovations.

Each potential consumer innovation in the heat-pump, pellet-burning, and solar technologies was next subjected to scrutiny by domain experts. In each field three independent experts were used: one expert represented academia and was specialized in the technology area, one represented consumer inventors, and one represented the specific renewables industry. The use of expert evaluations to verify and evaluate the characteristics of consumer innovations is common practice in user-innovation research (e.g., Franke et al., 2006; Hienerth et al., 2014) as in-depth domain knowledge is required for assessing the often non-patented user projects. The use of external experts to assess the consumer projects reduced the number to 213 consumer projects that improved the technology, its ease of use, its purpose of use, its energy efficiency, or its price for the consumer.

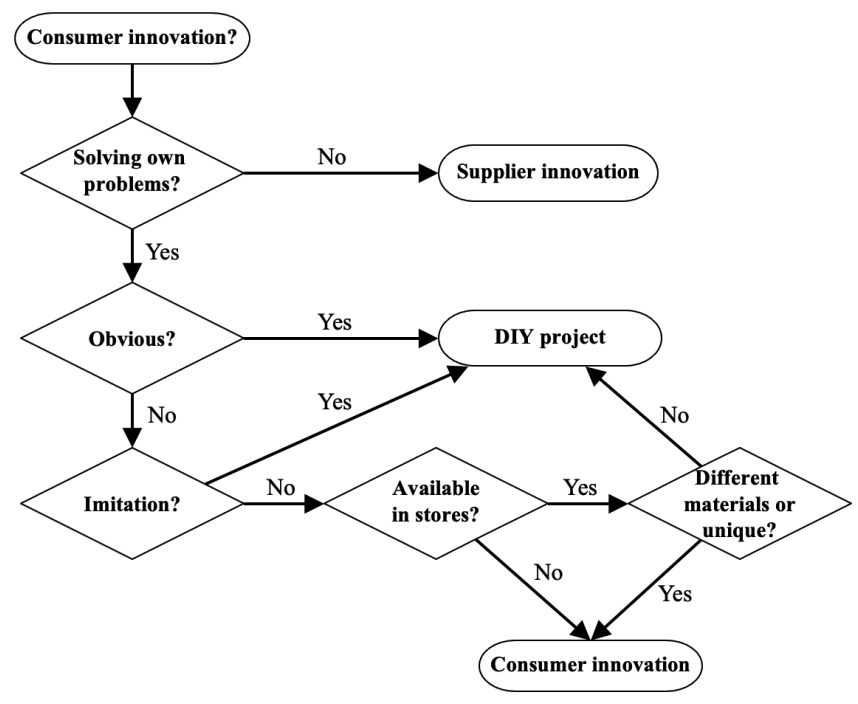

Figure A.2 Criteria for user innovations. 
In addition to its innovativeness, the expert evaluators also assessed each consumer project with regard to its ease of implementation for other competent users, its diffusion potential to the existing housing and device stock in Finland, and its direct energy-saving potential. We discuss these evaluations in Chapter 3.

To assess the diffusion of these 213 consumer innovations, we scanned the forums, as well as undertook broader internet searches, in order to discover if these solutions had been picked up or if they had a prior, parallel, or independent later existence of which both the consumer innovator and our expert evaluators might have been unaware. In this analysis we discarded 32 solutions on which the data available to us was too imprecise for clarifying whether or not they differed from projects found elsewhere. For each of the remaining 181 consumer projects, we proceeded to qualitatively analyze how the diffusion had taken place: whether the consumer innovation had proliferated through commercial channels, among peers, taken place as straight adoption, or whether it had included further innovative adaptations (see Chapter 3).

The net effect of this research approach is that we purposefully did not rely on self-reported consumer productions or non-verified self-estimations of their possible diffusion (De Jong et al., 2015). Our data consists of a set of contentanalyzed consumer innovations and content-verified instances of diffusion. This sets a heavy bias toward not detecting possible diffusion and also limits the amount of variables that could be established across the data set compared with user-innovation surveys where self-rating and self-assessment questions can be used to produce data for a great array of multivariate comparisons, yet offers also far less control over the verification of answers.

To further clarify the diffusion patterns of consumer innovations, we used statistical analysis where we used the results of expert evaluations for predicting whether a given consumer innovation should have diffused and examined them through cross-tabulations, bivariate analyses, and multivariate analyses. Both predicted and actual diffusion were measured with ordinal scales, but because of a heavy non-detection bias, these were simplified to a binary value in statistical analyses: no diffusion (0) or diffusion (1). All observations from both groups were independent and the distributions of the observations did not meet the normality criterion. For such circumstances, the most suitable bivariate tests were Fisher's exact test and the Mann-Whitney $U$ test since the data assumptions for the more common chi-square test and $t$-test did not apply (see Chapter 3). To further elaborate on these bivariate analyses, we conducted a multivariate analysis, which consisted of scatterplots, exact logistic regression, binomial logistic regression, and two-step cluster analysis. These confirmed the results from the bivariate analysis but did not shed any new light on the relations between dependent and independent variables.

\section{A.3 Virtual ethnography on Internet forums}

The study track on consumer innovation in S-RET was expanded to a broader study of the internet forums in which the innovating users were active soon 
after its beginning in 2011. This included several analysis stages. To understand more deeply how the internet forums functioned, we examined internet forum membership and postings and contents. We examined how new page views, members, and postings had accumulated in the lifespan of the forums, and then complemented this overview characterization with content analyses performed as part of our altogether 13 months of internet ethnography (conducted during 2011-2012 and complemented in 2017 and again in 2019). The ethnography included the base-level characterization of the contents of the forums' main subsections, consisting of a detailed coverage of 5-20 discussion threads per category and analysis of how members had sectioned and categorized the forums - as the forums are actively moderated and managed by peers, they hold a participant's own 'sociology' in how the contents and forms of communication are organized. These analyses helped to establish which forum areas (the categories for posts) had significant numbers of posts and how uniform the postings in each area were regarding form and content. In categories where more variety was found, we covered up to 20 threads in detail in order to understand the variety in the contents and the interaction activities. In categories that featured low variance in content and form, we settled on 5-10 threads (Gobo, 2007). Following this, we entirely covered those forum sections that were particularly variable and of interest to us, such as the above-detailed innovation data.

The ethnography also included 61 semi-structured interviews in order to gain personal accounts from the internet forum users and their activities. Of these, 47 were interviews with forum-active inventive consumers and five (5) were with firms that had collaborated with inventive consumers (see above on the innovation related parts of the interviews), and nine (9) were with users who had only adopted and not innovated in the S-RETs. Each interview was recorded and lasted for 30-120 minutes. We also manually examined the full user profiles of 115 forum users regarding posting types, post contents, how long they had been active, and how active they had been in the forums.

The posts and interviews were coded by one to three researchers. The initial coding focused on usage, procedures, and technology and design knowledge, which was constructed and shared inside the community (cf. Grabher and Ibert, 2014). For usage, we analyzed practices related to heating equipment (for example, advice on purchasing, installation, daily use, maintenance, general instructions, problem-solving, second-hand sales, and repurchasing). Procedural codes focused on forum activities and on discussions around laws, status, and building an open-source community. Technology- and designrelated coding analyzed the nature and make-up of inventions, the types of technology featured in the forums, support given and received, and members' learning and development pathways. In the second stage of analysis, these firstlevel thematic codes were clustered under major themes relating to user activities in sociotechnical change, which was followed by presentational coding and the resulting presentational narratives.

Statistics on all the major heat-pump internet forums (see Subsection 3.1 above) were obtained and analysed as well. As detailed statistical coverage for the 
whole duration of a forum's lifespan only existed for the largest of the forums, lampopumput.info, this forum was taken into the focus of analysis. These statistics cover postings, topics, readings, unique IP addresses, registered members, member posting profile distributions, and readings per discussion area.

\section{A.4 Analyzing the user activities in the heat-pump transition}

To gain an overview of the diffusion of heat pumps in Finland and its relation to the development of internet forums, we reanalyzed the above pre-analyzed data in a more zoomed-out manner and linked it to available published materials. We obtained the national energy production and usage statistics since the 1970s and the national sales statistics from the Finnish heat-pump industry association (SULPU) on all heat-pump types since the 1980s, complemented by analyses of heat pumps in Finland during 1980-2000 by Heiskanen et al. (2014b), Heiskanen et al. (2017a), and Lauttamäki (2018). To contextualize the heatpump proliferation still further, we analysed the reported studies conducted on Finnish heat pumps over the years, most importantly those by Heiskanen et al. $(2010,2011,2014,2017)$, as well as studies on local communities and the adoption of S-RET systems (Juntunen, 2014a, 2014b; Heiskanen et al., 2010, 2015). The Finnish case was contextualized through obtaining the European Heat Pump Association's annual comparison report (EHPA, 2018) and published literature on heat pumps in neighboring countries (e.g., Dzabo and Nyqvist, 2017). 


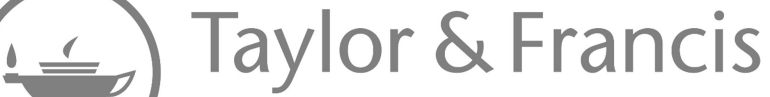

Taylor \& Francis Group

http://taylorandfrancis.com 


\section{References}

Abbott, A. 1988. The System of Professions: An Essay on the Division of Expert Labor. Chicago: University of Chicago Press.

Abbott, A. 1995. "Things of Boundaries." Social Research 63 (1): 857-882.

Abbott, A. 2005. "Linked Ecologies: States and Universities as Environments for Professions." Sociological Theory 23 (3): 245-274.

Abel, B., R. van Klaassen, L. Evers, and P. Troxler 2011. Open Design Now: Why Design Cannot Remain Exclusive. San Francisco: BIS.

Agarwal, B. 1983. "Diffusion of Rural Innovations: Some Analytical Issues and the Case of Wood-Burning Stoves." World Development 11 (4): 359-376.

Akera, A. 2001 "Voluntarism and the Fruits of Collaboration: The IBM User Group, Share." Technology and Culture 42 (4): 710-736.

Akera, A. 2007. "Constructing a Representation for an Ecology of Knowledge Methodological Advances in the Integration of Knowledge and its Various Contexts." Social Studies of Science 37: 413-441.

Akrich, M. 1992. "The Description of Technological Objects." In W.E. Bijker and J. Law (eds.), Shaping Technology—Building Society: Studies in Sociotechnical Change, 205-224. Cambridge, MA: MIT Press.

Alarcón Ferrari, C. and C. Chartier 2017. "Degrowth, Energy Democracy, Technology and Social-Ecological Relations: Discussing a Localised Energy System in Vaxjö, Sweden." Journal of Cleaner Production 197 (2): 1754-1765.

Alter, S. 2006. The Work System Method: Connecting People, Processes, and IT for Business Results. Larkspur, CA: Work System Press.

Appadurai, A. 1986. Social Life of Things: Commodities in Cultural Perspective. Cambridge:Cambridge University Press.

Arrow, K. 1962. "The Economic Implications of Learning by Doing." Review of Economic Studies 29: 155-173.

Attewell, P. 1992. "Technology Diffusion and Organizational Learning: The Case of Business Computing." Organization Science 3 (1): 1-19.

Bakardjieva, M. 2005. Internet Society: The Internet in Everyday Life. London: SAGE.

Baldwin, C., C. Hienerth, and E. von Hippel 2006. "How User Innovations Become Commercial Products: A Theoretical Investigation and Case Study." Research Policy 35: 1291-1313.

Baldwin, C. and E. von Hippel 2011. "Modeling a Paradigm Shift: From Producer Innovation to User and Open Collaborative Innovation.” Organization Science 22 (6): 1399-1417. 


\section{References}

Barnes, J. P. 2016. The Local Embedding of Technologies Through Community-Led Initiatives: The Case of Sustainable Energy. Brighton, UK: University of Sussex.

Becker, H. 1982. Art Worlds. Berkeley: University of California Press.

Becker, H. 1998. Tricks of the Trade. Chicago: University of Chicago Press.

Benkler, Y. 2006. The Wealth of Networks: How Social Production Transforms Markets and Freedom. New Haven: Yale University Press.

Bergek, A., M. Hekkert, S. Jacobsson, J. Markard, B. Sandén, and B. Truffer 2015. "Technological Innovation Systems in Contexts: Conceptualizing Contextual Structures and Interaction Dynamics." Environmental Innovation and Societal Transitions 16: 51-64. doi: 10.1016/j.eist.2015.07.003.

Berger, T., M. Hartmann, Y. Punie, and K. Ward, eds. 2006. Domestication of Media and Technology. Maidenhead, UK: Open University Press.

Berninger, K., R. Lovio, A. Temmes, P. Kivimaa, M. Jalas, and E. Heiskanen. 2017. Suomi seuraaville sukupolville - taloudellisten murrosten käsikirja (Finland to next generationshandbook of economic transitions). Helsinki: Into-Kustannus.

Bethwaite, F. 2008. Higher Performance Sailing. London: Adlard Coles Nautical.

Bijker, W. E. 1995. Of Bicycles, Bakelites, and Bulbs: Toward a Theory of Sociotechnical Change. Cambridge, MA: MIT Press.

Bijker, W., T. P. Hughes, and T. Pinch, eds. 1987. The Social Construction of Technological Systems: New Directions in the Sociology and History of Technology. Cambridge, MA: MIT Press.

Bjerknes, G., P. Ehn, and M. Kyng, eds. 1987. Computers and Democracy-A Scandinavian Challenge. Aldershot, England: Avebury.

Blumer, H. 1969. Symbolic Interactionism. Perspective and Method. Berkeley: University of California Press.

Bogers, M., A. Allan, and B. Bastian 2010. "Users as Innovators: A Review, Critique, and Future Research Directions." Journal of Management 36 (4): 857-875.

Botero, A. 2013. Expanding Design Spaces. Helsinki: Aalto University Press.

Botero, A., K.-H. Kommonen, and S. Marttila 2010. "Expanding Design Space: Design-InUse Activities and Strategies." In Proceedings of the Design Research Society 2010 Conference: Design and Complexity. Montreal, Canada: Design Research Society.

Botero, A. and S. Hyysalo 2013. "Ageing Together: Steps Towards Evolutionary Co-Design in Everyday Practices." CoDesign 9 (1): 37-54.

Bowker, G., and S. L. Star 1999. Sorting Things Out: Classification and Its Consequences. Cambridge, MA: MIT Press.

Bødger, K., F. Kensing, and J. Simonsen 2004. Participatory IT-Design. Cambridge, MA: MIT Press.

Braudel, F. 1995. Mediterranean and the Mediterranean World at the age of Philip 2. Translated by S. Reynolds. 2nd revised ed. Berkeley, CA: University of California Press.

Caird, S. and R. Roy 2008. "User-Centered Improvements to Energy Efficiency Products And Renewable Energy Systems: Research On Household Adoption And Use." International Journal of Innovation Management 12: 327-355.

Caird, S., R. Roy, and S. Potter 2012. "Domestic Heat Pumps in the UK: User Behaviour, Satisfaction and Performance." Energy Efficiency 5: 283-301.

Callon, M. 1991. "Techno-Economic Networks and Irreversibility." In J. Law (ed.), A Sociology of Monsters: Essays on Power, Technology, and Domination, 132-165. London: Routledge.

Callon, M. 1998. The Laws of the Markets. London: Blackwell Publishing. 
Callon, M., C. Méadel, and V. Rabeharisoa 2002. “The Economy of Qualities." Economics E Sociology 31: 194-217.

Cambrosio, A. and P. Keating 1995. Exquisite Specificity. Cambridge, MA: MIT Press.

Clarke, A. E. 1998. Disciplining Reproduction: Modernity, American Life and "the Problems of Sex'. Berkeley: University of California Press.

Clarke, A. E. 2005. Situational Analysis-Grounded Theory After the Postmodern Turn. Thousand Oaks: SAGE.

Clarke, A. E. and S. L. Star 2003. "Symbolic Interactionist Science, Technology, Information and Biomedicine Studies." In L. Reynolds and N. Herman (eds.), Handbook of Symbolic Interactionism, 539-574. Walnut Creek, CA: Alta Mira Press.

Cockburn, C. and S. Ormrod 1993. Gender and Technology in the Making. London: SAGE.

Cole, M. 1996. Cultural Psychology: A Once and Future Discipline. Cambridge: Harvard University Press.

Coombs, R., P. Saviotti, and V. Walsh, eds. 1987. Economics and Technological Change. Trenton: Rowman and Littlefield.

Creamer, E., G. Aiken, B. van Veelen, G. Walker, and P. Devine-Wright 2019. “Community Renewable Energy: What Does It Do? Walker and Devine-Wright (2008) Ten Years On." Energy Research \& Social Science 57: 101223.

de Haan, F. J. and J. Rotmans 2018. "A Proposed Theoretical Framework for Actors in Transformative Change." Technological Forecasting and Social Change 128: 275-286.

de Jong, J., E. von Hippel, F. Gault, J. Kuusisto, and C. Raasch 2015. "Market Failure in the Diffusion of Consumer-Developed Innovations: Patterns in Finland." Research Policy 44 (10): 1856-1865.

de Laet, M. and A. Mol 2000. "The Zimbabwe Bush Pump: Mechanics of Fluid Technology." Social Studies of Science 30 (2): 225-263.

Denzin, N. K. 1989. The Research Act. A Theoretical Introduction to Sociological Methods. 3rd ed. Upper Saddle River, NJ: Prentice Hall.

De Rubens, G., L. Noel, and B. Sovacool. 2018. "Dismissive and Deceptive Car Dealerships Create Barriers to Electric Vehicle Adoption at the Point of Sale." Nature Energy, 1.

de Vries, G. W., W. Boon, and A. Peine 2016. "User-Led Innovation in Civic Energy Communities." Environmental Innovation and Societal Transitions 19 (June): 51-65.

DeSantis, G. and M. S. Poole 1994. "Capturing the Complexity in Advanced Technology Use: Adaptive Structuration Theory." Organization Science 5 (2): 121-147.

Desouza, K., Y. Awazu, and A. Ramaprasad 2007. "Modifications and Innovations to Technology Artifacts.” Technovation 27 (4): 204-220.

Dewald, U. and B. Truffer 2011. "Market Formation in Technological Innovation Systems-Diffusion of Photovoltaic Applications in Germany." Industry and Innovation 18: 285-300.

Dewald, U. and B. Truffer 2012. "The Local Sources of Market Formation: Explaining Regional Growth Differentials in German Photovoltaic Markets." European Planning Studies 20 (3): 397-420.

Douthwaite, B., D. Keatinge, and J. Park. 2001. "Why Promising Technologies Fail: The Neglected Role of User Innovation during Adoption." Research Policy 30 (5): 819-836.

Durrant, R. 2014. Civil Society Roles in Transition: Towards Sustainable Food? Working Paper. Food Research Collaboration, SPRU University of Sussex.

Dzebo, A. and B. Nykvist 2017. "A New Regime and Then What? Cracks and Tensions in the Socio-Technical Regime of the Swedish Heat Energy System.” Energy Research and Social Science 29 (2017): 113-122. 
Edwards, P. N. 2010. A Vast Machine: Computer Models, Climate Data, and the Politics of Global Warming. Cambridge, MA: MIT Press.

Ehnert, F., F. Kern, S. Bogström, G. Leen, S. Maschmeyer, and M. Egermann 2017. "Urban Sustainability Transitions in a Context Of Multi-Level Governance: A Comparison of Four European States." Environmental Innovation and Societal Transitions 26: 101-116.

EHPA 2018. European Heat Pump Market Statistics, Report 2018. Brussels: European Heat Pump Association.

Engeström, Y. 1987. Learning by Expanding. An Activity-Theoretical Approach to Developmental Research. Helsinki: Orienta-Konsultit.

Engeström, Y. 2000. "Activity Theory as a Framework for Analyzing and Redesigning Work." Ergonomics 43 (7): 960-974.

Eurostat, 2019a. People in the EU - statistics on housing conditions Revision as of 16:57, 4 January 2019. Data extracted in May 2020.

Eurostat, 2019b. Energy prices in the EU 2019. Data extracted in May 2020.

Eurostat, 2020a. Electricity price statistics. Data extracted in May 2020.

Eurostat, 2020b. Greenhouse gas emissions per capita Tonnes of CO2 equivalent per capita. Extracted in May 2020.

Farla, J., J. Markard, R. Raven, and L. Coenen 2012. "Sustainability Transitions in the Making: A Closer Look at Actors, Strategies and Resources." Technological Forecasting and Social Change 79: 991-998.

Feenberg, A. 1999. Questioning Technology. London and New York: Routledge.

Finnish Energy 2020. Energia-alan vähähiilisyystiekartta 2020. [Low Carbon Road-Map for Energy-Sector 2020]. Helsinki: Finnish Energy Industries. https://energia.fi/files/4946/ Energia-alan_vahahiilisyystiekartta_2020.pdf (accessed 8 July 2020).

Fischer, C. S. 1992. America Calling: A Social History of the Telephone to 1940. Berkeley, CA: University of California Press.

Fleck, J. 1993a. "Configurations: Crystallizing Contingency." International Journal of Human Factors in Manufacturing 3 (1): 15-36.

Fleck, J. 1993b. "Innofusion: Feedback in the Innovation Process." In F. A. Stowell (ed.), Systems Science: Addressing Global Issues. London: Plenum.

Fleck, J. 1994. "Learning by Trying: The Implementation of Configurational Technology." Research Policy 23: 637-652.

Flichy, P. 2007. The Internet Imaginaire. Cambridge, MA: MIT Press.

Fligstein, N. and D. McAdam 2011. "Toward a General Theory of Strategic Action Fields." Sociological Theory 29 (1): 1-26.

Franke, N., E. Von Hippel, and M. Schreier 2006. "Finding Commercially Attractive User Innovations: A Test of Lead-User Theory." Journal of Product Innovation Management 23 (4): 301-315.

Franke, N. and M. Schreier 2010. "Why Customers Value Self-Designed Products: The Importance of Process Effort and Enjoyment." Journal of Product Innovation Management 27 (7): 1020-1031.

Freeman, C. 1979. "The Determinants of Innovation-Market Demand, Technology, and the Response to Social Problems." Futures 11 (3): 206-215.

Freeman, S. 2007. "The Material and Social Dynamics of Motivation: Contributions to Open Source Language Technology Development." Science Studies 20 (2): 55-77.

Freeman, S. 2011. Constructing a Community : Myths and Realities of the Open Development Model. Helsinki: University of Helsinki Press.

Freeman, S. 2015. "Immersed in Pellet Technology: Motivation Paths of Innovative DIYers." Outlines. Critical Practice Studies 16 (1): 54-80. 
Fujimura, J. 1996 Crafting Science: A Sociohistory of the Quest for the Genetics of Cancer. Cambridge, MA: Harward University Press.

Füller, J., M. Bartl, H. Ernst, and H. Mühlbacher 2006. "Community Based Innovation: How to Integrate Members of Virtual Communities into New Product Development." Electronic Commerce Research 6 (1): 57-73.

Galison, P. 1997. Image and Logic: A Material Culture of Microphysics. Chicago: University of Chicago Press.

Gambardella, A., C. Raasch, and E. von Hippel 2017. "The User Innovation Paradigm: Impacts on Markets and Welfare." Management Science 63 (5): 1450-1468.

Gardiner, P. and R. Rothwell 1985. "Tough Customers: Good Designs.” Design Studies 6 (1): 7-17.

Garud, R. and J. Gehman 2012. "Metatheoretical Perspectives on Sustainability Journeys: Evolutionary, Relational and Durational." Research Policy 41 (6): 980-995.

Galvin, R. and M. Sunikka-Blank 2014. "The UK Homeowner-Retrofitter as an Innovator in a Socio-Technical System.” Energy Policy 74 (November): 655-662.

Geels, F. W. 2002. "Technological Transitions as Evolutionary Reconfiguration Processes: A Multi-Level Perspective and a Case Study." Research Policy 31 (8): 1257-1274.

Geels, F. W. and J. Schot 2007. "Typology of Sociotechnical Transition Pathways." Research Policy 36: 399-417.

Geels, F.W., F. Kern, G. Fuchs, N. Hinderer, G. Kungl, J. Mylan, M. Neukirch, and S. Wasserman 2016. "The Enactment of Sociotechnical Transition Pathways: A Reformulated Typology and a Comparative Multi-Level Analysis of the German and UK Low-Carbon Electricity Transitions (1990-2014)." Research Policy 45 (4): 896-913.

Glaser, B. and A. Strauss 1967. The Discovery of Grounded Theory: Strategies for Qualitative Research. Chicago: Aldine Publishing Company.

Glaser, V., L. D’Adderio, and N. Pollock 2020. Biography of an algorithm. S.L.

Gobo, G. 2007. Sampling, "Representativeness and Generalizability." In C. Seale, G. Gobo, J. Gubrium, and D. Silverman (eds.), Qualitative Research Practice, 1-19. Sage Research Methods Online.

Government Programme, Marin Government Programme 2019. Inclusive and Competent Finland-A Socially, Economically and Ecologically Sustainable Society. Publications of the Finnish Government 2019: 25. Helsinki.

Grabher, G. and O. Ibert 2014. "Distance as Asset? Knowledge Collaboration in Hybrid Virtual Communities." Journal of Economic Geography 14: 97-123.

Guerin, D., B. Yust, and J. Coopet 2009. "Occupant Predictors of Household Energy Behavior and Consumption Change as Found in Energy Studies Since 1975." Family and Consumer Sciences Research Journal 29 (1): 48-80.

Haavisto, P. 2014. "Observing Discussion Forums and Product Innovation-A Way to Create Consumer Value? Case Heart-Rate Monitors.” Technovation 34: 215-222.

Habicht, H., P. Oliveira, and V. Shcherbatiuk 2013. "User Innovators: When Patients Set Out to Help Themselves and End Up Helping Many." Die Unternehmung 66 (3): 277-294.

Hakkarainen, L. and S. Hyysalo 2016. ”The Evolution of Intermediary Activities: Broadening the Concept of Facilitation in Living Labs." Technology \& Innovation Management Review 6 (1): 45-58.

Hargreaves, T., S. Hielscher, G. Seyfang, and A. Smith 2013. "Grassroots Innovations in Community Energy: The Role of Intermediaries in Niche Development." Global Environmental Change 23 (5): 868-880. 
Hasu, M. 2001. Critical Transition From Developers to Users. Helsinki: University of Helsinki Press.

Heiskanen, E., M. Johnson 0/0/00 0:00:00 AM, S. Robinson, E. Vadovics, and M. Saastamoinen 2010a. "Low-Carbon Communities as a Context for Individual Behavioural Change." Energy Policy 38: 7586-7595.

Heiskanen, E., S. Hyysalo, T. Kotro, and P. Repo 2010b. "Constructing Innovative Users and User-Inclusive Innovation Communities." Technology Analysis \& Strategic Management, 22 (4): 495-511.

Heiskanen, E. and R. Lovio 2010c. "User-Producer Interaction in Housing Energy Innovations." Journal of Industrial Ecology 14: 91-102.

Heiskanen, E., R. Lovio and M. Jalas 2011. "Path Creation for Sustainable Consumption: Promoting Alternative Heating Systems in Finland." Journal of Cleaner Production 19: 1892-1900.

Heiskanen, E., S. Hyysalo, M. Jalas, J.K. Juntunen, and R. Lovio 2014. "User Involvement and Radical Innovation: The Case of Heat Pumps in Finland." In S. Juninger and P. Christensen (eds.), Highways and Byways of Radical Innovation: The Perspective of Design, 178-191. Kolding: Allworth Press.

Heiskanen, E., M. Jalas, J. Rinkinen, and P. Tainio 2015. "The Local Community as a 'Low-Carbon Lab': Promises and Perils." Environmental Innovation and Societal Transitions 14 (March): 149-164.

Heiskanen, E., R. Lovio, and K. Louhija 2017a. "Miten uusi teknologia tulee uskottavaksi: esimerkkinä maalämpö suomessa [How New Technology Becomes Credible: Case Ground Source Heat Pumps in Finland]." Liiketaloudellinen aikakauskirja 14 (4): 277-298.

Heiskanen, E., H. Nissilä, and P. Tainio 2017b. "Promoting Residential Renewable Energy via Peer-to-Peer Learning." Applied Environmental Education \& Communication 16(2): 105-116.

Heiskanen, E., E-L. Apajalahti, K. Matschoss, and R. Lovio 2018. "Incumbent Energy Companies Navigating the Energy Transitions: Strategic Action or Bricolage?" Environmental Innovation and Societal Transitions 28: 57-69.

Heiskanen, E., S. Laakso, and K. Matchoss 2019. "Finnish Energy Policy in Transition.” In F Fahy, G Goggins and C Jensen Energy Demand (eds.), Challenges in Europe: Implications for Policy, Planning and Practice, 127-136. Dortrecht: Springer.

Helgesson, C.-F. and H. Kjellberg 2009. "Practical use: On Agency and the Use of Science and Technology." In E. Baraldi, H. Håkanson, F. Prenkert, and A. Waluschewski (eds.), Use of Science and Technology in Business: Exploring the Impact of Using Activity for Systems, Organizations and People. Bingley, UK: Emerald Publishing Group.

Heljo, J. and H. Laine 2005. "Sähkölämmitys Ja Lämpöpumput Sähkönkäyttäjinä Ja Päästöjen Aiheuttajina Suomessa. Näkökulma Ja Malli Sähkönkäytön Aiheuttamien CO2-Ekv Päästöjen Arviointia Varten [Heat-pumps as Electricity Users and Emission Source in Finland]." Tampere: Tampereen Teknillinen yliopisto.

Helminen, P., S. Mäkinen and S. Hyysalo 2018. "Organizational Adoption of the Lead User Method: A Follow-Up Study." International Journal of Business Excellence 12 (4): 508-536. Hess, D.J. 1997. Science Studies—An Advanced Introduction. New York: NYU Press.

Hess, D.J. 2005. "Technology-and Product-Oriented Movements: Approximating Social Movement Studies and Science and Technology Studies." Science, Technology, E Human Values 30 (4): 515-535.

Hess, D.J. 2016. "The Politics of Niche-Regime Conflicts: Distributed Solar Energy in the United States." Environmental Innovation and Societal Transitions 19: 42-50. 
Hienerth, C. 2006. "The Commercialization of User Innovations: The Development of the Rodeo Kayak Industry.” RED Management 36 (3): 273-94.

Hienerth, C., E. von Hippel, and M. B. Jensen 2014. "User Community vs. Producer Innovation Development Efficiency: A First Empirical Study." Research Policy 43 (1): 190-201.

Hine, C. 2007. "Multi-sited Ethnography as a Middle Range Methodology for Contemporary STS.” Science, Technology, \& Human Values 32 (6): 652-671.

Hoogma R., R. Kemp, J. Schot, and B. Truffer 2002. Experimenting for Sustainable Transport Futures. The Approach of Strategic Niche Management. London: EF\&N Spon.

Howells, J. 2006. "Intermediation and the Role of Intermediaries in Innovation." Research Policy 35: 715-28.

Höyssä, M. and S. Hyysalo 2009. "The Fog of Innovation: Innovativeness and Deviance in Developing New Clinical Testing Equipment.” Research Policy 38 (6): 984-993.

Hutchins, E. 1995. Cognition in the Wild. Cambridge, MA: MIT Press.

Hyysalo, S. 2004. Uses of Innovation. Wristcare in the Practices of Engineers and Elderly. Helsinki: Helsinki University Press.

Hyysalo, S. 2006. "The Role of Learning-By-Using in the Design of Healthcare Technologies: A Case Study." The Information Society 22 (2): 89-100.

Hyysalo, S. 2007." Versions of Care Technology." Human Technology, An International Journal on Humans in ICT Environments 3 (2): 228-247.

Hyysalo, S. 2009. "User Innovation and Everyday Practices: Micro-Innovation in Sports Industry Development.” RED Management 39 (3): 247-258.

Hyysalo, S. 2009. "Learning for Learning Economy and Social Learning." Research Policy 38 (4): 726-735.

Hyysalo, S. 2010. Health Technology Development and Use: From Practice Bound Imagination to Evolving Impacts. London: Routledge.

Hyysalo, S. and J. Lehenkari 2002. "Contextualizing Power in Collaborative Design." In T. Binder, J. Gregory, and I. Wagner (eds.), Proceedings of Participatory Design Conference 2002, 93-104. Malmö, Sweden: Computer Professionals for Social Responsibility.

Hyysalo, S., J.K. Juntunen, and S. Freeman 2013a. "User Innovation in Sustainable Home Energy Technologies." Energy Policy 55: 490-500.

Hyysalo, S., J.K. Juntunen, and S. Freeman 2013b. "Internet Forums and the Rise of the Inventive Energy User." Science and Technology Studies 26 (1): 25-51.

Hyysalo, S., P. Helminen, S. Mäkinen, M. Johnson, J.K. Juntunen, and S. Freeman 2015. "Intermediate Search Elements and Method Combination in Lead-User Searches." International Journal of Innovation Management 19, 1-37.

Hyysalo, S. and L. Hakkarainen 2014. "What Difference Does a Living Lab Make? Comparison of Two Health Technology Innovation Projects." CoDesign 10 (3-4): 191-208.

Hyysalo, S. and S. Usenyuk 2015. "The User Dominated Technology Era: Dynamics of Dispersed Peer-Innovation.” Research Policy 44: 560-576.

Hyysalo, S., T.E. Jensen, and N. Oudshoorn, eds. 2016a. The New Production of Users: Changing Innovation Collectives and Involvement Strategies. New York: Routledge.

Hyysalo, S., T.E. Jensen, and N. Oudshoorn 2016b. "Introduction to the New Production of Users." In S. Hyysalo, T.E. Jensen, and N. Oudshoorn (eds.), The New Production of Users: Changing Innovation Collectives and Involvement Strategies. New York: Routledge.

Hyysalo, S., P. Repo, P. Timonen, L. Hakkarainen, and E. S 2016c. "Diversity and Change of User Driven Innovation Modes in Companies." International Journal of Innovation Management 20 (2). 
Hyysalo, S., M. Johnson, and J.K. Juntunen 2017. "The Diffusion of Consumer Innovation in Sustainable Energy Technologies." Journal of Cleaner Production 162 (supplement): S70-S82. Hyysalo, S. and J.K Juntunen 2018. "User Innovation and Peer Assistance in Small Scale Renewable Energy Technologies." In D.J. Davidson and M. Gross (eds.), Oxford Handbook of Energy and Society, 361-380. Oxford: Oxford University Press.

Hyysalo, S., J.K.Juntunen, and M. Martiskainen 2018. "Energy Internet Forums as Acceleration Phase Transition Intermediaries." Research Policy 47 (5): 872-885.

Hyysalo, S., N. Pollock, and R. Williams 2019a. "Method Matters in the Social Study of Technology: Investigating the Biographies of Artifacts and Practices." Science and Technology Studies 32 (3): 2-25.

Hyysalo, S., J. Lukkarinen, P. Kivimaa, R. Lovio, A. Temmes, M.Hildén, T. Marttila, K. Auvinen, S. Perikangas, A. Pyhälammi, J. Peljo, K. Savolainen, L. Hakkarainen, M. Rask, K. Matschoss, T. Huomo, A. Berg, and M. Pantsar 2019b. "Developing Policy Pathways: Redesigning Transition Arenas for Mid-Range Planning." Sustainability 11 (3): 603.

Hyysalo, S., T. Marttila, S. Perikangas, and K. Auvinen 2019c. "Codesign for Transitions Governance: A Mid-Range Pathway Creation Toolset for Accelerating Sociotechnical Change." Design Studies 63: 181-203.

IEA (International Energy Agency) 2018. The World Energy Outlook 2018. Paris: Organisation for Economic Cooperation and Development (OECD).

The Intergovernmental Panel on Climate Change (IPCC) (2018). "Global Warming of 1.5C. An IPCC Special Report on the Impacts of Global Warming of 1.5C Above PreIndustrial Levels and Related Global Greenhouse Gas Emission Pathways." In P. Zhai, H. O. Portner, D. Roberts, J. Skea, P. R. Shukla, A. Pirani, V. Masson-Delmotte et al. (eds.), The Context of Strengthening the Global Response to the Threat of Climate Change, Sustainable Development, and Efforts to Eradicate Poverty (in press).

Jalas, M., S. Hyysalo, E. Heiskanen, R. Lovio, A. Nissinen, M. Mattinen, J. Rinkinen, J.K. Juntunen, P. Tainio, and H. Nissilä 2017. "Everyday Experimentation in Energy Transition: A Practice-Theoretical View." Journal of Cleaner Production 169 (December): 77-84.

Janasik, N. 2011. A Cognitive and Pragmatic Account of Innovation: Domesticating the Probiotic Gefilus in Finland. Espoo: Aalto University Press.

Jeppesen, L. and L. Frederiksen 2006. "Why Do Users Contribute to Firm-hosted User Communities? The Case of Computer-controlled Music Instruments." Organization Science 17 (1): 45-63.

Jeppesen, L. and M. Molin 2003. "Consumers as Co-developers: Learning and Innovation Outside the Firm. " Technology Analysis \& Strategic Management, 15 (1): 363-383.

Jensen, T. E. 2012. "Intervention by Invitation: New Concerns and New Versions of the User in STS.” Science \& Technology Studies 25 (1): 13-36.

Johansson, P. 2017. A Silent Revolution—The Swedish Transition Towards Heat Pumps, 19702015. Stockholm, Sweden: KTH Royal Institute of Technology.

Johnson, M. 2013. How Social Media ChangesUser-Centred Design-Cumulative and Strategic User Involvement with Respect to Developer-User Social Distance. Espoo: Aalto University Press.

Johnson, M., S. Hyysalo, and S.Tamminen 2010. "The Virtuality of Virtual Worlds, or What We Can Learn from Playacting Horse Girls and Marginalized Developers." Symbolic Interaction 33 (4): 603-633.

Johnson, M., H. Mozaffar, G.M. Campagnolo, S. Hyysalo, N. Pollock, and R. Williams 2014. "The Managed Prosumer: Evolving Knowledge Strategies in the Design Of Information Infrastructures." Information, Communication and Society 17 (7): 795-813. 
Jørgensen, U. 2012. "Mapping and Navigating Transitions-The Multi-Level Perspective Compared with Arenas Of Development." Research Policy 41(6): 996-1010.

Judson, E.P., S. Bell, H. Bulkeley, G. Powells, and S. Lyon 2015. "The Co-construction of Energy Provision and Everyday Practice: Integrating Heat Pumps in Social Housing in England." Science \& Technology Studies 28 (3): 26-53.

Juntunen, J.K. 2014a. "Domestication Pathways of Small-Scale Renewable Energy Technologies.” Sustainability: Science, Practice, E Policy 10 (1): 1206-1230.

Juntunen, J.K. 2014b. Prosuming Energy-User Innovation and New Energy Communities in Renewable Micro-Generation. Helsinki: Aalto University Press.

Juntunen, J and S. Hyysalo 2015 "Renewable Micro-generation of Heat and ElectricityReview on Common and Missing Socio-Technical Configurations." Renewable \& Sustainable Energy Reviews 49: 857-870.

Kallinikos, J. 2004 "Farewell to Constructivism: Technology and Context-Embedded Action." In C. Avgerou, C. Ciborra, and F. Land (eds.), The Social Study of Information and Communication Technology: Innovation, Actors, and Contexts. Oxford: Oxford University Press.

Kanger, L. and J. Schot 2016. "User-Made Immobilities: A Transitions Perspective." Mobilities 11: 598-613.

Kanger, L., F.W. Geels, B. Sovacool, and J. Schot 2019. "Technological Diffusion as a Process of Societal Embedding: Lessons from Historical Automobile Transitions for Future Electric Mobility." Transport Research D Transport and Environment. 71, 47-66.

Karnøe, P. and R. Garud 2012. "Path Creation: Co-Creation of Heterogeneous Resources in the Emergence of the Danish Wind Turbine Cluster." European Planning Studies 20: 733-752.

Kemp, R., J. Schot, and R. Hoogma 1998. "Regime Shifts to Sustainability through Processes of Niche Formation: The Approach of Strategic Niche Management." Technology Analysis \& Strategic Management 10: 175-195.

Kivimaa, P., W. Boon, S. Hyysalo, and L. Klerkx 2019a. “Towards a Typology of Intermediaries in Transitions: A Systematic Review.” Research Policy 48 (4): 1062-1075.

Kivimaa, P., S Hyysalo, W Boon, L Klerkx, M Martiskainen, and J Schot 2019b. "Passing the Baton: How Intermediaries Advance Sustainability Transitions in Different Phases." Environmental Innovation and Societal Transitions 31: 110-125.

Klerkx, L and N. Aarts 2013. "The Interaction of Multiple Champions in Orchestrating Innovation Networks: Conflicts and Complementarities." Technovation 33: 193-210.

Kline, S. J. and N. Rosenberg 1986. "An Overview of Innovation." In R. Landau and N. Rosenberg (eds.), The Positive Sum Strategy: Harnessing Technology for Economic Growth. Washington, DC: National Academy Press.

Klein, H. K. and D. L. Kleinman 2002. "The Social Construction of Technology: Structural Considerations." Science, Technology \& Human Values 27 (1): 28-52.

Kline, R. and T. Pinch 1996. "Users as Agents of Technological Change: The Social Construction of the Automobile in the Rural United States." Technology and Culture 37: 763-795.

Köhler, J., F.W. Geels, F. Kern, J. Markard, A. Wieczorek, F. Alkemade, F. Avelino, A. Bergek, F. Boons, L. Fünfschilling, D. Hess, G. Holtz, S. Hyysalo, K. Jenkins, P. Kivimaa, M. Martiskainen, A. McMeekin, M.S. Mühlemeier, B. Nykvist, E. Onsongo, B. Pel, R. Raven, H. Rohracher, B. Sandén, J. Schot, B. Sovacool, B. Turnheim, D. Welch, and P. Wells 2019. "An Agenda for Sustainability Transitions Research: State of the Art and Future Directions." Environmental Innovation and Societal Transitions 31: $1-32$. 


\section{References}

Kohtala, C. 2017. "Making 'Making' Critical: How Sustainability is Constituted in Fab Lab Ideology.” Design Journal 20 (3): 375-394.

Kohtala, C. and S. Hyysalo 2015. "Anticipated Environmental Sustainability of Personal Fabrication." Journal of Cleaner Production 99: 333-344.

Kohtala, C., S. Hyysalo, and J. Whalen (2020). "A Taxonomy of User' Active Design Engagement in 21st Century." Design Studies 67: 27-54.

Kopytoff, I. 1986. "The Cultural Biography of Things: Commoditization as Process, in The Social Life of Things." In A. Appadurai (ed.), Social Life of Things: Commodities in Cultural Perspective, 64-94. Cambridge: Cambridge University Press.

Kuznetsov, S. and E. Paulos 2010. "Rise of the Expert Amateur: DIY Projects, Communities, and Cultures." In Proceedings of the 6th Nordic Conference on Human-Computer Interaction: Extending Boundaries, 295-304. New York: AC Med.

Latour, B. 1987. Science in Action: How to Follow Scientists and Engineers through Society. Cambridge, MA: Harvard University Press.

Latour, B. 2005. Reassembling the Social. Oxford, UK: Oxford University Press.

Lauttamäki, V. 2018. Geoenergia kiinteistöjen lämmitysratkaisujen markkinoilla Suomessa energiakriisien ajoista 2030-luvulle [Geoenergy in Building Heat Markets in Finland from the Times of Energy Crises to 2030]. Turku: University of Turku Press.

Lauttamäki, V. and S. Hyysalo 2019. "Empirical Application of the Multi-Level Perspective: Tracing the History of Ground-Source Heat Pumps in Finland." Sustainability: Science, Practice, and Policy 15 (1): 82-103.

Lave, J., and E. Wenger 1991. Situated Learning: Legitimate Peripheral Participation. Cambridge, UK: Cambridge University Press.

Law J. 2007. "Making a Mess With Method.” In W. Outhwaite and S.P. Turner (eds.), The Sage Handbook of Social Science Methodology, 595-606. London: SAGE.

Leivo, V. and R. Lindberg 2008. "Lämmitysenergian säästö ja energiansäästöpotentiaalit [Heat energy savings and energy saving potential].” Tekniikan maailma. Rakennusmaailma 08/2008.

Leminen, S. 2015. Living Labs as Open Innovation Networks-Networks, Roles and Innovation Outcomes. Espoo: Aalto University Press.

Levi, G. 1988. Inheriting Power. The Story of an Exorcist. Chicago: University of Chicago Press.

Lie, M. and K. Sørensen, eds. 1996 Making Technology Our Own? Domesticating Technology into Everyday Life. Oslo: Scandinavian University Press.

Lovio, R., P. Mickwitz, and E. Heiskanen 2011. "Path Dependence, Path Creation and Creative Destruction in the Evolution of Energy Systems." In R. Wustenhagen and R. Wuebker (eds.), The Handbook of Research on Energy Entrepreneurship, 274-290. Northhampton, UK: Edward Elgar.

Lukkarinen, J., T. Marttila, H. Saarikoski, K. Auvinen, M. Faehnle, S. Hyysalo, HL. Kangas, S. Lähteenoja, L. Peltonen, and M. Salo 2020. "Taloyhtiöistä tulevaisuuden energiatuottajia-Muntospolut vuoteen 2035 ja murrosareena tiedon yhteistuotannon menetelmänä [Turning Housing Companies as Future Energy Producers-Change Pathways to Year 2035 and Transition Arena as a Method for Coproduction of Knowledge]." Suomen ympäristökeskuksen raportteja 39/2020. Helsinki: SYKE.

Lundqvist, P. 2008. "The Heat Pump in the Refurbishment of Existing Buildings, Perspectives from Sweden." In Proc. Joint REHVA/AICARR Workshop on the Refurbishment of Existing Buildings. Milan: Academic Conferences Publishing.

Lundvall, B.-Å. 1988. "Innovation as an Interactive Process: From User-Producer Interaction to the National System of Innovation." In G. Dosi, C. Freeman, R. R. 
Nelson, G. Silverberg, and L. Soete (eds.), Technological Change and Economic Theory. London: Printer Publishers Ltd.

Lundvall, B.-Å. and A. L. Vinding 2005. "Product Innovation and Economic TheoryUser-Producer Interaction in the Learning Economy." In J. L. Christensen (ed.), Product Innovation, Interactive Learning and Economic Performance. Amsterdam: Elsevier Science.

Lüthje, C. 2004. "Characteristics of Innovating Users in a Consumer Goods Field: An Empirical Study of Sport-Related Product Consumers." Technovation 24 (9): 683-695.

Luthje, C., C. Herstatt, and E. von Hippel 2005. "User Innovators and 'Local' Information: The Case of Mountain Biking." Research Policy 34 (6): 951-965.

Lutzenhiser, L. 1993. "Social and Behavioral Aspects of Energy Use." Annual Review of Energy and the Environment 18 (1): 247-289.

Mackay, H., C. Carne, P. Beynon-Davies, and D. Tudhope. 2000. "Reconfiguring the User: Using Rapid Application Development." Social Studies of Science 30 (5): 737-757.

MacKenzie, D.A. and J. Wajcman, eds. 1999. The Social Shaping of Technology, 2nd ed. Buckingham: Open University Press.

Marchand, R. 1998. "Customer Research as Public Relations: General Motors." In S. Strasser, C. McGoverns, and M. Judt (eds.), Getting and Spending: European and American Consumer Societies in the Twentieth Century, 85-110. Cambridge, UK: Cambridge University Press.

Marchi, G., C. Giachetti, and P. de Gennaro. 2011. "Extending Lead-User Theory to Online Brand Communities: The Case of the Community Ducati." Technovation 31(8): 350-361.

Marcus, G. E. 1995. Ethnography in/of the World System: The Emergence of Multi-Sited Ethnography. Annual Review of Anthropology 24: 95-117.

Martiskainen, M. 2014. Developing Community Energy Projects: Experiences from Finland and the UK. PhD Thesis. University of Sussex, Brighton.

Martiskainen, M., and P. Kivimaa 2018. "Creating Innovative Zero Carbon Homes in the United Kingdom-Intermediaries and Champions in Building Projects." Environmental Innovation and Societal Transitions 26: 15-31.

Matschoss, K. and E. Heiskanen 2018. "Innovation Intermediary Challenging the Energy Incumbent: Enactment of Local Socio-Technical Transition Pathways by Destabilisation of Regime Rules.” Technology Analysis \& Strategic Management, 30 (12) 1455-1469.

Matschoss, K. and E. Heiskanen 2017. "Making It Experimental in Several Ways: The Work of Intermediaries in Raising the Ambition Level in Local Climate Initiatives." Journal of Cleaner Production 169: 85-93.

Mattinen, M., A. Nissinen, S. Hyysalo, and J.K. Juntunen 2014. "Energy Use and Greenhouse Gas Emissions of Air-Source Heat Pump and Innovative Ground-Source Air Heat Pump in Cold Climate." Journal of Industrial Ecology 19 (1): 61-70.

McLaughlin, J., P. Rosen, D. Skinner, and A. Webster 1999. Valuing Technology: Organisations, Culture, and Change. London: Routledge.

Meelen, T., B. Truffer, and T. Schwanen 2019. "Virtual User Communities Contributing to Upscaling Innovations in Transitions: The Case of Electric Vehicles." Environmental Innovation and Societal Transitions 31: 96-109.

Meelen, T., K. Frenken, and S. Hobrink 2019. "Weak Spots for Car-Sharing in the Netherlands? The Geography of Socio-Technical Regimes and the Adoption of Niche Innovations." Energy Research \& Social Science 52: 132-143.

Miettinen, R. 1993. "Methodological Issues of Studying Innovation Related Networks." Working papers of VTT Group for Technology Studies 4. 


\section{References}

Miettinen, R. 2003. National Innovation System Scientific Concept or Political Rhetoric? Helsinki: Sitra.

Mignon, I. and A. Bergek 2016. "System-and Actor-Level Challenges for Diffusion of Renewable Electricity Technologies: An International Comparison." Journal of Cleaner Production 128: 105-115.

Miller, D. and D. Slater 2007. "Moments and Movements in the Study of Consumer Culture: A discussion Between Daniel Miller and Don Slater." Journal of Consumer Culture 7 (5): 5-23.

Mol, A. 2002. The Body Multiple: Ontology in Medical Practice. Durham: Duke University Press.

Monteiro, E., N. Pollock, O. Hanseth, and R. Williams 2013. "From Artefacts to Infrastructures." Computer Supported Co-operative Work 22 (4-6): 575-607.

Moore, G. 2001. Crossing the Chasm. New York: Harper Collins.

Motiva 2010. ELVARI, jälkiasennetun ilmalämpöpumpun vaikutus energiankäyttöön https ://www.motiva.fi/files/3960/Jalkiasennetun_ilmalampopumpun_vaikutus_energia nkayttoon.pdf (accessed 13 March 2019).

Motiva 2012. ELVARI ilmalämpöpumpun energiataloudellinen käyttö https://www.mot iva.fi/files/3472/Ilmalampopumpun_energiataloudellinen_kaytto.pdf (accessed 13 March 2019).

Mozaffar, H. 2016. "User Communities as Multifunctional Spaces: Innovation, Collective Voice, Demand Articulation, Peer Informing and Professional Identity (and More)." In S. Hyysalo, T. Jensen, and N. Oudshoorn (eds.), The New Production of Users: Changing Innovation Collectives and Involvement Strategies, 219-248. Routledge: New York.

Murto, P., M. Jalas, J.K. Juntunen, and S. Hyysalo 2019a. ”Energy Retrofits are a Great Idea, But Not So Easy to Purchase: Barriers Posed by Complicated Calculability and Nascent Markets." Energy Policy 132: 955-964.

Murto, P., M. Jalas, J.K. Juntunen, and S. Hyysalo 2019b. Complexity Management Devices and Strategies Intermediating Energy Retrofits. Renewable \& Sustainable Energy Reviews 114: 109294.

Murto, P., S. Hyysalo, J.K. Juntunen, and M. Jalas 2020. "Capturing the Micro-Level of Intermediation in Transitions: Comparing Ethnographic and Interview Methods." Environmental Innovation and Societal Transitions 36: 406-417.

Nicolini, D. 2012. Practice Theory, Work and Organization: An Introduction. Oxford, UK: Oxford University Press.

Nielsen, K. H. 2016 "How User Assemblage Matters: Constructing Learning by Using in the Case of Wind Turbine Technology in Denmark, 1973-1990." In S. Hyysalo, T.E. Jensen, and N. Oudshoorn (eds.), New Production of Users, 101-122. Routledge: New York.

Nielsen, K. R., L. A. Reisch, and J. Thøgersen 2016. "Sustainable User Innovation from a Policy Perspective: A Systematic Literature Review." Journal of Cleaner Production 133: 65-77.

Noble, D. 1984. Forces of Production: A Social History of Machine Tool Automation. New York: A. A. Knopf.

Nyborg, S. 2015. "Pilot Users and Their Families: Inventing Flexible Practices in the Smart Grid." Science \& Technology Studies 28 (3): 54-80.

Nyborg, S. and I. Røpke 2015. "Heat Pumps in Denmark-From Ugly Duckling to White Swan." Energy Research and Social Science 9: 166-177.

Nygrén, N., P. Kontio, J. Lyytimäki, V. Varho, and P. Tapio 2015. "Early Adopters Boosting the Diffusion of Sustainable Small-Scale Energy Solutions." Renewable and Sustainable Energy Reviews 46: 79-87. 
Nye, M., L. Whitmarsh, and T. Foxon 2010. "Socio-Psychological Perspectives on the Active Roles of Domestic Actors in Transition to a Lower Carbon Electricity Economy." Environment and Planning Part A 42 (3): 697-714.

Okamura, K., M. Fujimoto, W. Orlikowski, and J. Yates 1994. "Helping CsCw Applications Succeed: The Role of Mediators in the Context of Use." In Proceedings of PDC 1994. New York, NC: ACM Press.

Ornetzeder, M. and H. Rohracher 2006. "User-Led Innovations and Participation Processes: Lessons from Sustainable Energy Technologies.” Energy Policy 34 (2): 138-150.

Ornetzeder, M. and H. Rohracher 2013. "Of Solar Collectors, Wind Power, and Car Sharing: Comparing and Understanding Successful Cases of Grassroots Innovations." Global Environmental Change 23: 856-867.

Oudshoorn, N., E. Rommes, and M. Stienstra 2004. "Configuring the User as Everybody: Gender and Design in Information and Communication Technologies." Science, Technology \& Human Values 29 (1): 30-63.

Oudshoorn, N. and T. Pinch, eds. 2003. How Users Matter. The Co-Construction of Users and Technologies. Cambridge, MA: MIT Press.

Pahkala, T., H. Uimonen, and V. Väre. 2018. Joustava ja asiakaskeskeinen sähköjärjestelmäÄlyverkkotyöryhmän loppuraportti [Flexible and customer centred electricity systemthe final report of smart grid working group]. Työ- ja elinkeinoministeriön julkaisuja 2018:33: Helsinki.

Piller, F. and J. West 2014. "Firms, Users, and Innovation: An Interactive Model of Coupled Open Innovation." In H. Chesbrough and W. Vanhaverbeke (eds.), New Frontiers in Open Innovation. Oxford, UK: Oxford University Press.

Palm, J and S.J. Darby 2014."The Meanings of Practices for Energy Consumption-a Comparison of Homes and Workplaces." Science \& Technology Studies 27 (2): 72-92.

Pfaffenberger, B. 1992. "Technological Dramas." Science, Technology and Human Values 17 (3): 282-312.

Pipek, V. and V. Wulf 2009. "Infrastructuring: Toward an Integrated Perspective on the Design and Use of Information Technology." Journal of the Association for Information Systems 10 (5): Article 1.

Pitta, D.A. and D. Fowler 2005. "Internet Community Forums: An Untapped Resource for Consumer Marketers." Journal of Consumer Marketing 22 (5): 265-274.

Pollock, N and S. Hyysalo 2014. "The Business of Being a User: The Role of the Reference Actor in Shaping Packaged Enterprise System Acquisition and Development." MIS Quarterly 38 (2): 473-496.

Pollock, N. and R. Williams 2016. How Industry Analysts Shape the Digital Future Oxford, UK: Oxford University Press.

Pollock, N. and R. Williams 2008. Software and Organizations: The Biography of the Packaged Enterprize System, Or, How SAP Conquered the World. Routledge, London.

Prahalad, C. K. and V. Ramaswamy 2004. The Future of Competition: Co-creating Unique Value with Customers. Boston: Harward Business School Press.

Prügl, R. and M. Schreier 2006. "Learning from Leading-edge Customers at The Sims: Opening up the Innovation Process Using Toolkits." RED Management 36 (3): 237-250.

Raasch, C. and E. von Hippel. 2014. "Innovation Process Benefits: The Journey as Reward." MIT Sloan School of Management Magazine research Feature, Fall 2013.

Raven, R. P., E. Heiskanen, R. Lovio, M. Hodson, and B. Brohmann 2008. "The Contribution of Local Experiments and Negotiation Processes to Field-Level Learning in Emerging (Niche) Technologies: Meta-Analysis of 27 New Energy Projects in Europe." Bulletin of Science, Technology \& Society 28 (6): 464-477. 
Ribes D. and J. Polk 2015. "Organizing for Ontological Change: The Kernel of a Research Infrastructure." Social Studies of Science 45(2): 214-241.

Rinkinen, J. and M. Jalas 2017. "Moving Home: Houses, New Occupants and the Formation of Heating Practices." Building Research \& Information 45 (3): 293-302.

Rip, A. and R. Kemp 1998. “Technological Change.” In S. Rayner and E.L. Malone (eds.), Human Choice and Climate Change, Vol. 2, 327-399. Columbus, OH: Battelle Press.

Rogers, E. 2010. Diffusion of Innovations, 5th ed. Avon, MA: Simon and Schuster.

Rohracher, H. 2003. "The Role of Users in the Social Shaping of Environmental Technologies." Innovation 16 (2): 177-196.

Rohracher, H. and H. Köhler 2019. "Households as Infrastructure Junctions in Urban Sustainability Transitions: The Case of Hot Water Metering." Urban Studies 56 (11): 2372-2386.

Romero-Rubio, C. and J. de Andrés Díaz 2015. "Sustainable Energy Communities: A Study Contrasting Spain and Germany." Energy Policy 85: 397-409.

Rosenberg, N. 1982. Inside the Black Box: Technology and Economics. Cambridge: Cambridge University Press.

Rosenberg, N. 1979. Perspectives on Technology. Cambridge: Cambridge University Press.

Rotmans, J., R. Kemp, and M. van Asselt 2001. "More Evolution than Revolution: Transition Management in Public Policy." Foresight 3: 15-31.

Ruostetsaari, I. 2010. Energiavalta: Eliitti Ja Kansalaiset Munttuvilla Energiamarkkinoilla. Tampere University Press. [Energy power: Elite and citizens in changing energy markets].

Ruostetsaari, I. 2020. "From Consumers to Energy Citizens: Finns' Readiness for Demand Response and Prosumerism in Energy Policy Making." International Journal of Energy Sector Management 14 (16): 1157-1175.

Russell, S. 1986. "The Social Construction of Artefacts: A Response to Pinch and Bijker." Social Studies of Science 16: 331-346.

Russell, S. and R. Williams. 2002. "Social Shaping of Technology: Frameworks, Findings and Implications for Policy with Glossary of Social Shaping Concepts." In K. Sorensen and R. Williams (eds.), Shaping Technology, Guiding Policy: Concepts, Spaces and Tools, 37-132. Cheltenham, UK: Edward Elgar.

Sawhney, M., G. Verona, and E. Prandelli 2005. "Collaborating to Create: The Internet as a Platform for Customer Engagement in Product Innovation." Journal of Interactive Marketing 19 (4): 4-17.

Safarzyńska, K., K. Frenken, and J. van den Bergh 2012. "Evolutionary Theorizing and Modeling of Sustainability Transitions." Research Policy 41 (6): 1011-1024.

Schwartz-Cowan, R. 1983. More Work for Mother. The Ironies of Household Technology from the Open Heart to the Microwave. New York: Basic Books

Schot, J., L. Kanger, and G. Verbong 2016. "The Roles of Users in Shaping Transitions to New Energy Systems.” Nature Energy 1 (5): 16054.

Schuler, D. and A. Namioka, eds. 1993. Participatory Design: Principles and Practices. Hillsdale, NJ: Lawrence Erlbaum Associates.

Scott-Kemmis, D. and M. Bell 2010. "The Mythology of Learning-By-Doing in World War II Airframe and Ship Production." International Journal of Technological Learning, Innovation and Development 3 (1).

Seyfang, G. 2010. "Community Action for Sustainable Housing: Building a Low-Carbon Future." Energy Policy 38 (12): 7624-7633.

Shah, S. and M. Tripsas 2007. "The Accidental Entrepreneur: The Emergent and Collective Process of User Entrepreneurship.” Strategic Entrepreneurship Journal 1 (1-2): 123-140. 
Shove, E., M. Watson, M. Hand, and J. Ingram 2007. The Design of Everyday Life. Oxford: Berg Publishers.

Shove, E., M. Pantzar, and M. Watson 2012. The Dynamics of Social Practice: Everyday Life and How It Changes. Thousand Oaks: SAGE.

Shove, E. 2010. "Beyond the ABC: Climate Change Policy and Theories of Social Change." Environment and Planning, Part A, 42 (6): 1273-1285.

Shove, E. and A. Warde 2002. "Inconspicuous Consumption: The Sociology of Consumption, Lifestyles and the Environment." Sociological Theory and the Environment: Classical Foundations, Contemporary Insights 230: 51.

Silvast, A., H., Hänninen, and S. Hyysalo 2013. "Energy in Society: Energy Systems and Infrastructures in Society." Science \& Technology Studies 26 (3): 3-13.

Silvast, A., R. Williams, S. Hyysalo, K. Rommetveit, and C. Raab 2018. "Who 'Uses' Smart Grids? The Evolving Nature of User Representations in Layered Infrastructures." Sustainability $10(10): 3738$.

Silvast, A and M.J. Virtanen 2019. "An Assemblage of Framings and Tamings: Multi-sited Analysis of Infrastructures as a Methodology." Journal of Cultural Economy 12 (6): 461-477.

Silverstone, R., E. Hirsch, and D. Morley 1992. "Information and Communication Technologies and the Moral Economy of the Household." In R. Silverstone and E. Hirsch (eds.), Consuming Technologies: Media and Information in Domestic Spaces, 115-131. London: Routledge.

Smith, A. 2012. "Civil Society in Sustainable Energy Transitions." In G. Verbong and D. Loorbach (eds.), Governing the Energy Transition: Reality, Illusion or Necessity?, 180-202. New York: Routledge.

Smith, A., M. Fressoli, and H. Thomas. 2014. "Grassroots Innovation Movements: Challenges and Contributions." Journal of Cleaner Production 63: 114-124.

Smith, A., F. Kern, R. Raven, and B. Verhees. 2014. "Spaces for Sustainable Innovation: Solar Photovoltaic Electricity in the UK." Technological Forecasting and Social Change 81: 115-130.

Smith, A., M. Fressoli, D., Abrol, E. Arond, and A. Ely 2016a. Grassroots Innovation Movements. Routledge: New York.

Smith, A., T. Hargreaves, S. Hielscher, M. Martiskainen, and G. Seyfang 2016b. "Making the Most of Community Energies: Three Perspectives on Grassroots Innovation." Environment and Planning, Part A 48: 407-432.

Sørensen, K. and R. Williams 2002. Shaping Technology, Guiding Policy: Concepts, Spaces and Tools. Cheltenham, UK: Edward Elgar.

Sørensen, K.H. 1996. "Learning Technology, Constructing Culture." STS Working Papers 18/96. Norwegian University of Science and Technology.

Spinardi, G. and R. Slayton 2015. "Greener Aviation Take-off (Delayed): Analysing Environmental Transitions with the Multi-Level Perspective." Science \& Technology Studies 28 (1).

Star, S.L. and A. Strauss 1999. "Layers of Silence, Arenas of Voice: The Ecology of Visible and Invisible Work." Computer Supported Cooperative Work 8 (1): 9-30.

Star, S. L. and J. Griesemer 1989. "Institutional Ecology, 'Translations' and Boundary Objects: Amateurs and Professionals in Berkeley's Museum of Vertebrate Zoology, 1907-1939." Social Studies of Science 19: 387-420.

Star, S. L. and K. Ruhleder 1996. "Steps Toward an Ecology of Infrastructure: Design and Access for Large Information Spaces.” Information Systems Research 7 (1): 111-134.

Star, S. L. 1989a. Regions of the Mind: Brain Research and the Quest for Scientific Certainty. Stanford, CA: Stanford University Press. 
Star, S. L. 1989b. "The Structure of Ill-Structured Solutions: Boundary Objects and Heterogeneous Distributed Problem Solving." In M.N. Huhns and L. Gasser (eds.), Distributed Artificial Intelligence. Menlo Park: Morgan Kaufman.

Star, S. L. 1995. Ecologies of Knowledge: Work and Politics in Science and Technology. New York: SUNY Press.

Star, S. L. 1996. "Working Together: Symbolic Interactionism, Activity Theory, and Information Systems." In Y. Engeström and Middelton D. (eds.), Cognition and communication at work 296-318. Cambridge: Cambridge University Press.

Statistics Finland 2018. Suomen virallinen tilasto (SVT): Kasvihuonekaasut [verkkojulkaisu]. ISSN=1797-6049. 2018, Suomen kasvihuonekaasupäästöt 2018. Helsinki: Tilastokeskus [viitattu: 7.7.2020]. Saantitapa: http://www.stat.fi/til/khki/2018/khki_2018_2019-05 -23_kat_001_fi.html

Statistics Finland 2019. Suomen virallinen tilasto (SVT): Rakennukset ja kesämökit [verkkojulkaisu]. ISSN=1798-677X. 2019, Liitetaulukko 1. Rakennukset, asunnot ja henkilöt talotyypin ja kerrosluvun mukaan 31.12.2019. Helsinki: Tilastokeskus [viitattu: 7.7.2020]. Saantitapa: http://www.stat.fi/til/rakke/2019/rakke_2019_2020-05-27_t au_001_fi.html

Statistics Finland 2020a. Suomen virallinen tilasto (SVT): Asunnot ja asuinolot [verkkojulkaisu]. ISSN=1798-6745. Helsinki: Tilastokeskus [viitattu: 7.7.2020]. Saantitapa: http://www.stat.fi/til/asas/tau.html

Statistics Finland 2020b. Energiatilasto 2019.

Statistics Finland 2020c. Suomen virallinen tilasto (SVT): Asumisen energiankulutus [verkkojulkaisu]. ISSN=2323-3273. Helsinki: Tilastokeskus [viitattu: 7.7.2020]. Saantitapa: http://www.stat.fi/til/asen/index.html

Steinheimer, M., U. Trick, and P. Ruhrig 2012. "Energy Communities in Smart Markets for Optimisation of Peer-to-Peer Interconnected Smart Homes." In 2012 8th International Symposium on Communication Systems, Networks \& Digital Signal Processing (CSNDSP), $1-6$.

Stewart, J. 2003. "The Social Consumption of Information and Communication Technologies. ICTs: Insights from Research in the Appropriation and Consumption of New ICTs in the Domestic Environment." Cognition, Technology and Work 5: 4-14.

Stewart, J. 2007. "Local Experts in the Domestication of Information and Communication Technologies." Information, Communication \& Society 10: 547-569.

Stewart, J. and S. Hyysalo 2008. "Intermediaries, Users and Social Learning in Technological Innovation." International Journal of Innovation Management 12 (3): 295-325.

Stewart, J. and R. Williams 2005. "The Wrong Trousers? Beyond the Design Fallacy: Social Learning and the User." In H. Rohracher (ed.), User Involvement in Innovation Processes. Strategies and Limitations From a Socio-Technical Perspective. Munich: Profil-Verlag.

Strauss, A. 1993. Continual Permutations of Action. New York: Aldine de Gruyter.

Strauss, A. 1978. "A Social World Perspective." In N. Denzin (ed.), Studies in Symbolic Interaction, 119-128. Greenwich: JAI Press.

Strauss, Anselm, and Juliet Corbin 1990. Basics of Qualitative Research. London: Sage.

Suchman, L. 1987. Plans and Situated Actions. Cambridge, UK: Cambridge University Press. Suchman, L. 1995. "Making Work Visible." Communications of the ACM 38 (9): 56-64.

Suchman, M. C. 1995. "Managing Legitimacy: Strategic and Institutional Approaches." Academy of Management Review 20 (3): 571-610.

[dataset] SULPU, 2018. Heat pump statistics 2018. https://www.sulpu.fi/tilastot (accessed 13 March 2019). (Includes detailed data-set on heat-pump sales in Finland obtained 
directly from the association, 2018 figures added in from Sulpu 2018 separate sales figures)

Szymanski, M. and J. Whalen 2011. Making Work Visible. Cambridge: Cambridge University Press.

TM Rakennusmaailma (2018) TM on testannut lämpöpumppuja jo 10 vuotta. [TM has been testing heat-pumps for ten years] https://rakennusmaailma.fi/lampopumppuja-on -testattu/.

Tapscott, D. and A. Williams 2008. Wikinomics: How Mass Collaboration Changes Everything. New York: Penguin.

Torrance, A. and E. Von Hippel 2016. "Protecting the Right to Innovate: Our 'Innovation Wetlands."” In Hyysalo et al. (eds.), New Production of Users, 44-74, New York: Routledge.

Torrey, C., E.F. Churchill, and D.W. McDonald 2009. "Learning How: The Search for Craft Knowledge on the Internet." In CHI '09 Proceedings of the SIGCHI Conference on Human Factors in Computing Systems, 1371-1380. New York: ACM.

Torrey, C., D. McDonald, B. Schilit, and S. Bly 2007. "How-To Pages: Informal Systems of Expertise Sharing." In ECSCW 2007 Proceedings of the 10th European Conference on Computer-Supported Cooperative Work, 391-410. Limerick, Ireland: Springer.

Truffer, B. 2003. "User-led Innovation Processes: The Development of Professional Car Sharing by Environmentally Concerned Citizens." Innovation: The European Journal of Social Science Research 16 (2): 139-154.

Tyre, M. and E. von Hippel 1997. "The Situated Nature of Adaptive Learning in Organizations.” Organization Science 8 (1): 71-83.

Usenyuk, S., S. Hyysalo and J. Whalen 2016. "Proximal Design: Users as Designers of Mobility in the Russian North." Technology and Culture 57 (4): 866-908.

Van de Ven, A., D. Polley, R. Garud, and S. Venkataraman. 1999. The Innovation Journey. Oxford: Oxford University Press.

Van de Ven, A. and M. Scott Poole 2005. "Alternative Approaches for Studying Organizational Change.” Organization Studies 26 (9): 1377-1404.

Van Mierlo, Barbara, and Pieter J. Beers 2020. "Understanding and Governing Learning in Sustainability Transitions: A Review." Environmental Innovation and Societal Transitions 34: 255-269

Van Poeck, Katrien, Leif Östman, and Thomas Block. 2020. “Opening up the Black Box of Learning-by-Doing in Sustainability Transitions." Environmental Innovation and Societal Transitions 34: 298-310.

Verhaegh, S., E. Van Oost, and N. Oudshoorn 2016. "Innovation in Civil Society: The Socio-Material Dynamics of Community Innovation." In S. Hyysalo, T. Jensen, and N. Oudshoorn (eds.), The New Production of Users: Changing Innovation Collectives and Involvement Strategies, 193-218. New York: Routledge.

von Hippel, E. 1976. "The Dominant Role of Users in the Scientific Instrument Innovation Process." Research Policy 5 (3): 212-239.

Von Hippel, E. 1988. The Sources of innovation. New York: Oxford University Press.

Von Hippel, E. 2005. Democratizing Innovation. Cambridge, MA: MIT Press

Von Hippel, E. 2016. Free Innovation. Cambridge, MA: MIT Press.

Von Hippel, E. and M. Tyre. 1995. "How 'Learning by Doing' Is Done: Problem Identification in Novel Process Equipment." Research Policy 24 (1): 1-12.

Von Hippel, E., H. DeMonaco, and J. de Jong. 2017. "Market Failure in the Diffusion of Clinician-Developed Innovations: The Case of off-Label Drug Discoveries." Science and Public Policy 44 (1): 121-131. 
von Krogh, G. and E. von Hippel 2003. "Special Issue on Open Source Software Development." Research Policy 32: 1149-1157.

Von Hippel, E. and G. Von Krogh 2016. "Crossroads-Identifying Viable 'Need-Solution Pairs': Problem Solving without Problem Formulation.” Organization Science 27 (1): 207-221.

Voss, A., M. Hartswood, R. Procter, M. Rouncefield, R. S. Slack, and M. Büscher, eds. 2009. Configuring User-Designer Relations - Interdisciplinary Perspectives. London: Springer.

Voss, A., R. Procter, R. S. Slack, M. Hartswood, R. Procter, and M. Rouncefield. 2009. "Design as and for Collaboration: Making Sense of and Supporting Practical Action." In A. Voss, M. Hartswood, R. Procter, M. Rouncefield, R. S. Slack, and M. Büscher (eds.), Configuring User-Designer Relations-Interdisciplinary Perspectives, 38-51. London: Springer.

Walker, G. and P. Devine-Wright 2008. "Community Renewable Energy: What Should It Mean?” Energy Policy 36: 497-500.

Weber, K. M. and H. Rohracher 2012. "Legitimizing Research, Technology and Innovation Policies for Transformative Change: Combining Insights from Innovation Systems and Multi-Level Perspective in a Comprehensive 'Failures' Framework." Research Policy 41 (6): 1037-1047.

Wenger, E. 1998. Communities of Practice. Cambridge: Cambridge University Press.

Williams, R. and D. Edge 1996. "The Social Shaping of Technology." Research Policy 25 (6): 865-899.

Williams, R., J.K. Stewart, and R. Slack 2005. Social Learning and Technological Innovation. Cheltenham: Edward Elgar.

Winner, L. 1980. "Do Artifacts have Politics?” Deadalus 109 (1): 121-136.

Woolgar, S. 1991. "Configuring the User: The Case of Usability Trials." In J. Law (ed.), A Sociology of Monsters : Essays on Power, Technology, and Domination, 58-99. London: Routledge.

Voß, J-P. and N. Amelung 2016. "Innovating Public Participation Methods: Technoscientization and Reflexive Engagement." Social Studies of Science 46 (5): 749-772.

Zogg, M. 2008. "History of Heat Pumps-Swiss Contributions and International Milestones." https://www.osti.gov/etdeweb/biblio/21381633 (accessed 13 March 2019).

Zolfagharian, M., B. Walrave, R. Raven, and A. Romme 2019. "Studying Transitions: Past, Present, and Future." Research Policy 48 (9): 103788. 


\section{Index}

$\star \star$ Page numbers in bold reference tables.

$\star \star$ Page numbers in italics reference figures.

Abbott, A. 21, 22

acceleration and embedding phase 96,

119-120; heat-pump transition

104-105, 132

acceleration-phase internet peer

discussions 86

acquisition, supporting with internet

communities 65-70

actor-level relationships of user

activities 111

adaptation 36, 98, 107-108, 119, 129

adjustment, user activities related to heat pumps 107-108

adoption 25-26, 97-98, 106-107, 110-111, 118-119, 126-129; mass adoption 116 ; by peers $49-50$; heat pumps (Finland) 102-103

agency in transition pathways 32

air-source heat pump (ASHP) 37-41, 49, 101, 102, 104, 106

air-to-water heat pump (ASWHP) 38, 50-51

analysis units, BOAP (biographies of artifacts and practices) 25-29

ANT 22

arenas for social action 23, 114-115

ASHP (air-source heat pump) 37-41, 49, 101, 102, 104, 106

ASWHP (air-to-water heat pump) 38, $50-51$

biographies of artifacts and practices (BOAP) 7

BOAP (biographies of artifacts and practices) $7,9,18-21,23-24,133-135$; analysis units, observation units, and data 25-29; within-case sampling 29-33; S-RET internet forums 94-95; theoretical case sampling 29-33

boundary infrastructure 73

building legitimacy 119, 129

championing 119, 120, 129; intermediation 108-109

citizen initiatives 125

citizen users, innovation 56

citizens, sustainable sociotechnical change 3-7; see also users

Clarke, A. E. 23

climate policy, user-driven energy and 138-141

commercial diffusion 53

communities 62-65, 124-125; differences between wide-reaching innovation communities and local communities 126-129; hybrid communities 127-129; see also internet communities community creation, transitions 109 community energy 89

community energy groups 65 ; versus distributed and dispersed energy communities 87-91

comparative perspective on different energy communities 86-91

configurational movements 114-116, 130-133

consumer innovation 35-37; diffusion in S-RETs 46-56; entrepreneurship 54-55

consumer inventions: expert evaluations of 45-46; Finnish S-RET systems 37-45 consumers 35 
data collection, user innovations and diffusion of user innovation 146-149

data in studies of technology adoption and innovation, BOAP (biographies of artifacts and practices) 25-29

demand articulation, internet communities 81-82

DeMonaco, H. 36

design spaces under conditions of high variation in user needs 57

Devine-Wright, P. 89

diffusion 57-58; consumer innovation and 35-37, 58-60; hybrid communities 127129; innovative diffusion 126; S-RETs 46-56; of user innovations 146-149

digital connectivity 36

distributed energy communities, versus local community energy groups 87-91

distribution of consumer innovation diffusion 53

do-it-yourself renaissance 6 domestication pathways 5

early adopters 4

ecological sociology 21

ecologies of interconnected actors 19 , 21-24

ecology 21

ecology of actors 113-115

ecology of intermediaries in Finnish heatpump market (2010-2014) 83-85

ecology of intermediation 82

EHPA (European Heat Pump Association) 144, 151

energy policy, Finland 143-144

energy prices, Finland 144-145

energy systems in Finland, adoption and innovation 142-146

energy technology business, user-driven energy 135-138

engagement, online discussion forums $70-73$

entrepreneurship, consumer innovation 54-55

entrepreneurers, diffusion of consumer innovation 48-49

environmental policy, user-driven energy 139-141

ethnographic observation 31

European Heat Pump Association (EHPA) 144,151

evidence of realized value, internet communities 81

examples of community energy $\mathbf{8 9}$ expansion, theoretical case sampling 30

expert evaluations 45-46, 47, 148; peer

diffusion 55-56

external experts 148

Finland: arena for heat pumps 114-115; energy policy 143-144; energy prices 144-145; energy system and S-RET adoption and innovation 142-146; heatpump transition 100-106; user activities related to heat pumps 106-112

Finnish Borewell Association (Poratek) 102 Finnish Heat Pump Association (SULPU) 102

Finnish S-RET systems, consumer inventions: ASHP (air-source heat pump) 37-41; GSHPs (ground-source heat pumps) 41-43; pellet wood burning systems 43-45

Fisher's exact test 54, 56, 149

focal interest-context interrelations 30

focal perspective, theoretical case sampling 30

focalization of research 23-24

forums see internet communities

fostering critical discourse on technology, internet communities 86-87

framing effects 16-18

Freeman, S. 73

Geels, F. W. 32-33, 97

Grassroots innovation 34

ground-source heat pumps (GSHPs) 41-43, 101, 102, 104, 106; user inventions 42

growth patterns, S-RET internet forums 78-80

GSHPs (ground-source heat pumps) 41-43, 101, 102, 104, 106; user inventions 42

heat-pump forums: complementing market information 80-81; evidence of realized value 81 ; learning technology and deepening engagement 70-73; peer assistance for user projects 73-78; peerto-peer internet communities within ecology of user-side intermediaries 82-86; supporting acquisition and usership 65-70, 76-77

heat-pump transition: in Finland, user contributions (1980-2018) 100-106; user activities 106-112, 151

Heiskanen, E. 6, 151

Helgesson, C.-F. 69

hollow-arch bias 17-19 
home consumption 5

home multimedia 5

hybrid communities 127-129

\section{Ibert, O. 75}

independent peer knowledge, internet communities 80-81

innofusion 6, 36, 130-131

innovation 117

innovation by users, transitions 108

innovation communities 125

innovation process studies 29

innovation studies, S\&TS and 124-125

innovation surveys 25

innovative adaptation by peers, diffusion of consumer innovation 50

innovative diffusion 126

intensification 119; focal perspective 30

interaction arenas 46

intermediation 108-109, 119, 129

internet communities 62-65, 91-93, 116, 126, 146; comparative perspective on different energy communities 86-94; complementing market information 80-81; contributing to demand articulation and technical improvement 81-82; heat-pump transition 103-104; learning technology and deepening engagement 70-73; peer assistance for user projects 73-78; peer-to-peer internet communities within ecology of user-side intermediaries 82-86; providing evidence of realized value and counter-evidence against claims of poor performance 81; S-RET internet forums' growth patterns 78-80; supporting acquisition and usership 65-70; virtual ethnography 149-151

internet ethnography 150

interrelations 111

interstices 19, 21-24

invisible work 2

\section{Jääsähkö Oy 48}

Kanger, L. 121

Kjellberg, H. 69

Kuznetsov, S. 75

learning technology, online discussion forums 70-73

legitimacy building 110, 119, 120, 129

local communities, versus wide-reaching diverse communities 126-129 local community energy communities 109 , 126-129; versus distributed and dispersed energy communities 87-91

local experts 5, 69

Lumituuli 88

maintenance, transitions 109

Mann-Whitney U test 55, 149

mapping energy communities 90

market creation 109-110, 119

markets, internet communities, complementing market information 80-81

mass adoption 116

materiality 20

measures of the association between independent and dependent variables $\mathbf{5 4}$

Meelen, T. 97

memberships 23

MLP 22

Motiva 139

mountaineering search 147

multiple enacted contexts 20

multiple memberships 23

multi-sited ethnography 18

no diffusion $51-52$

non-visibility 100

Nye, M. 93

observation units, BOAP (biographies of artifacts and practices) 25-29

online discussion forums, diffusion of consumer innovation 48

OpenOffice.org 73

open-source projects, OpenOffice.org 73

organizational diffusion 26

Paulos, E. 75

peer assistance, for user projects, internet communities 73-78

peer diffusion 52, 58-59; expert evaluations 55-56

peers 62,146

peer-to-peer internet communities within ecology of user-side intermediaries 82-86

pellet wood burning systems 43-45

performance, evidence of realized value, internet communities 81

peripheral participation 23

Pollock, N. 28, 30

premature sense of closure 16-18

process questions 28

project championing, transitions 108-109 


\section{Index}

R\&D expenditure 35

recalibrating transitions research 121-122

residential energy use 34

revealing innovation to companies,

diffusion of consumer innovation 49

routine use 106-107

S\&TS (science and technology studies) 9, 63; innovation studies and 124-125; transition studies and 129-133

scales 19

Schot, J. 32, 97, 99, 121

science and technology studies (S\&TS) 9, 63; innovation studies and 124-125;

transition studies and 129-133

sensitizing concepts 22

series of configurational movements, transitions 112-118, 120, 130-131

small-scale renewable energy technology (S-RET) 4-5

Smith, A. 99, 111, 122

snapshot bias 16-17

social studies of technology (SST) 15

social value, user innovations 125-126

sociotechnical change 1-3, 117; approach

to researching 7-9; citizens in 3-7

sociotechnical configuration 113-114

sociotechnical voluntarism 22

Solar Energy Cooperative Green Point

Batensteinbad Woerden 88

solar panels 51-52

S-RETs (small-scale renewable energy technology) 4-5, 33, 34, 36, 37, 125-126, 133, 137; adoption and innovation 142-146; diffusion of consumer innovation 46-56, 58-60; Finnish S-RET systems see Finnish S-RET systems; internet communities 91; internet forums' growth patterns $78-80$

SST (social studies of technology) 15

stabilization phase 97; heat-pump transition 105-106

Star, S. L. 23

Stewart, J. 66, 69

straight adoptions, diffusion of consumer innovation 49-50

strategic ethnography 30

Strauss, A. 23

SULPU (Finnish Heat Pump

Association) 102

survey-based innovation studies 25

sustainability transitions research 8 sustainable sociotechnical change, citizens in 3-7

systems change view, affecting market and shaping technology 78-86

take-off phase 96; heat-pump transition 103 technical improvements, internet communities 81-82

technology adoption research 25

technology proliferation, configuration model 117

technology type 114

theoretical case sampling, BOAP (biographies of artifacts and practices) 29-33

transition framing 97,129

transition pathways 32

transition studies, S\&TS 129-133

transitions 96-100, 120; heat-pump transition in Finland (1980-2018) 100-106; as a series of configurational movements 112-118

transitions research, recalibrating 121-122

under-diffusion 36, 60

user activities: heat-pump transition 151; at transition process level 112

user activities related to heat pumps, Finland 106-112

user add-ons: ASHP (air-source heat pump) 40-41; GSHPs (ground-source heat pumps) 43; pellet wood burning systems $44-45$

user communities 119, 128-129

user consumers 99, 122

user contributions, to heat-pump transition, Finland (1980-2018) 100-106

user design: ASHP (air-source heat pump) 37-39; GSHPs (ground-source heat pumps) 41-42; pellet wood burning systems 43

user innovations 116, 119; criteria for 148; diffusion of user innovations 146-149; social value 125-126; transitions 108; user communities and 128-129

user innovators 110-111, 125

user intermediaries 99

user inventions 56; ASHP (air-source heat pump) 37-41; GSHPs (ground-source heat pumps) 41-43; pellet wood burning systems $43-45$

user modification and repurposing: ASHP (air-source heat pump) 40; GSHPs 
(ground-source heat pumps) 42; pellet wood burning systems 43-44

users 2, 35; approach to researching 7-9; role of 3-7; see also citizens users' engagement 118 usership 64; internet communities 65-70 user-driven energy: climate policy and

138-141; energy technology business 135-138

user-innovation communities 6 user-innovation research 7-8 user-legitimators 99, 122n 4 user-producers 99

user-run discussion forums 63 user-side intermediaries 5, 63

user-side intermediation 69

virtual ethnography, internet forums' growth patterns 149-151

Von Hippel, E. 2, 36, 127

Walker, G. 89

warm experts 5

Williams, R. 28, 30

within-case sampling, BOAP (biographies of artifacts and practices) 29-33

workplace ICTs 5

workplace information systems 5 


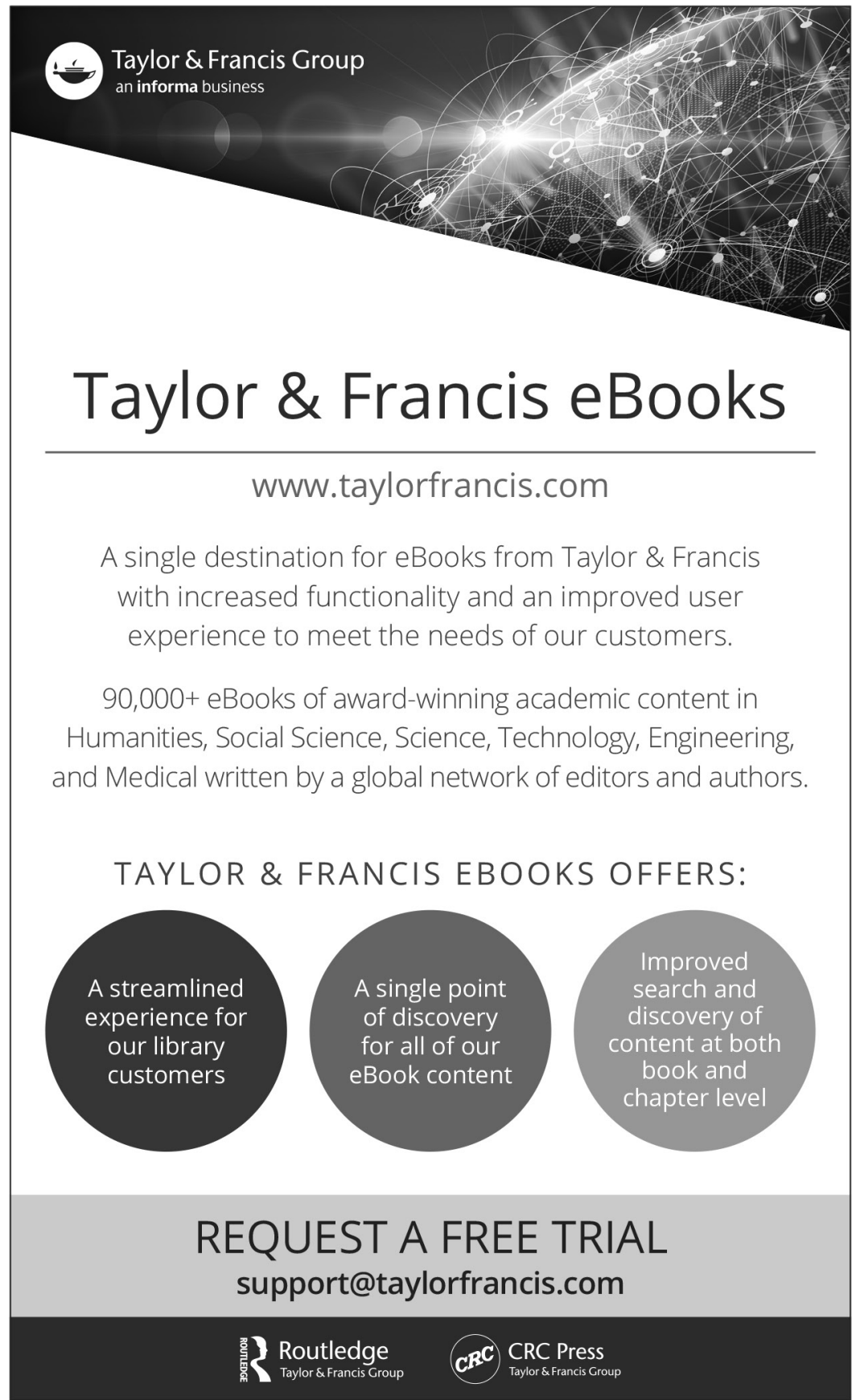

